\title{
H. H. DIXON
}

\section{UC-NRLF}

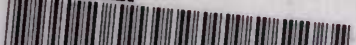

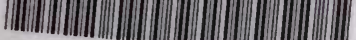

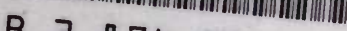

B 3874003 

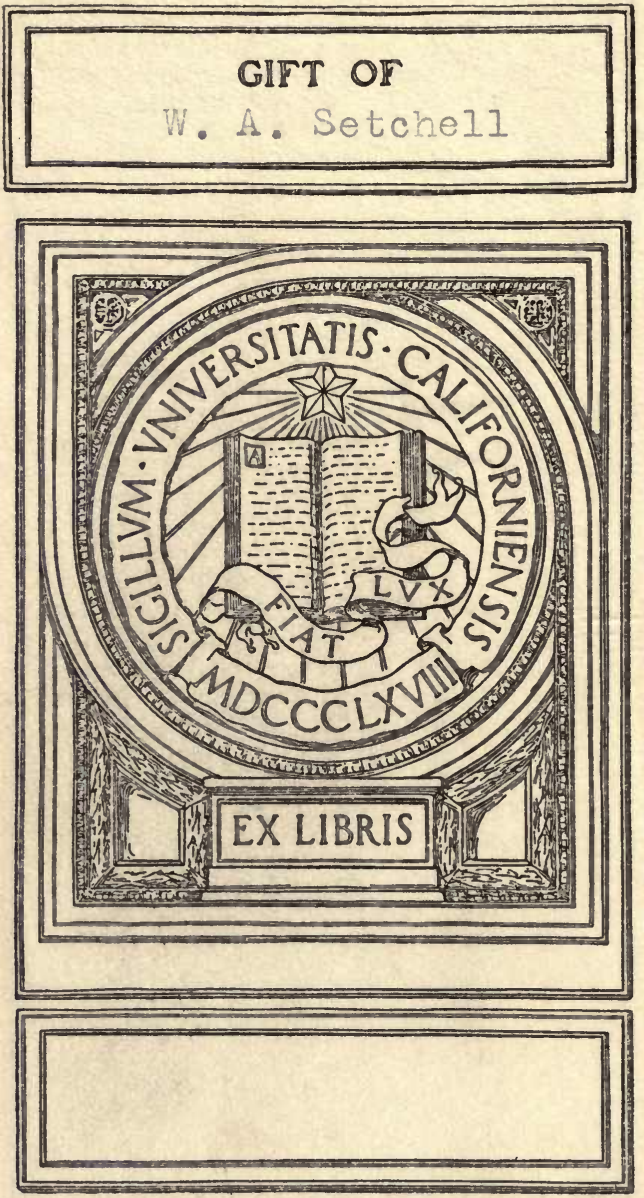

PRACTICAL PLANT BIOLOGY 
$180175 \% 94$

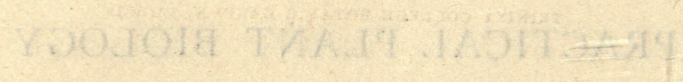




\section{PR ACTICAL}

\section{PLANT B IOLOGY}

A COURSE OF ELEMENTARY LECTURES ON THE GENERAL MORPHOLOGY AND PHYSIOLOGY OF PLANTS

BY

\section{HENRY H. DIXON, Sc.D., F.R.S.}

PROFESSOR OF BOTANY IN THE UNIVERSITY OF DUBLIN AND DIRECTOR OF TRINITY COLLEGE BOTANIC GARDEN, DUBLIN

WITH NINETY-FOUR ILLUSTRATIONS

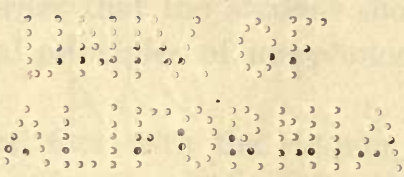

LONGMANS, GREEN AND CO.

39 PATERNOSTER ROW, LONDON, E.C. 4

55 FIFTH AVENUE, NEW YORK BOMBAY, CALCUTTA, AND MADRAS 
TADITDA 99

Y00.1018

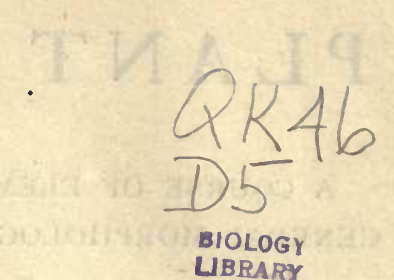

Gir g. An u' A Setched 


\section{PREFACE.}

THIs book consists of a series of thirty lectures designed as an Introductory Course in Botany for Medical and other Science Students. Practical work is outlined to illustrate each lecture.

At the outset the use of the microscope is treated, and special consideration is given to microscopic measurement and to the reconstruction of microscopic objects in the solid.

The plants chosen as subjects of study are necessarily limited in number. They have been selected, as fairly representing some of the more important subdivisions of the vegetable kingdom, and as being, at the same time, easy to procure. Attention is directed only to those points of their structure, life, history, and manner of living which are necessary in order that the student should appreciate some of the general principles of morphology, physiology, and evolution.

The lectures deal first with the simpler forms, among which are to be found some of the most important so far as general biology is concerned. They then pass on to the more complicated, and gradually lead the student to some knowledge of the development, structure, and physiology of the higher plants.

The arrangement of the course is the result of many years' teaching experience, and in its present form has been found 
to meet the requirements of elementary students and to interest them in the scientific and æsthetic beauty of plant biology.

I wish to acknowledge the help I have received from discussions with Prof. F. O. Bower, Sc.D., F.R.S., Prof. J. Joly, Sc.D., F.R.S., Mr. W. R. G. Atkins, O.B.E., Sc.D., and Mr. N. G. Ball, M.A. I am also greatly indebted to the last three for their kindness in reading the proofs.

H. H. D.

School of Botany, Trinity College,

Dublin, April, rg22. 


\title{
CONTENTS.
}

\author{
LECTURE I.
}

THE Microscope.

Names of the parts of a microscope-Microscopic preparation-Cells of epidermis of Tradescantia virginiana-Uses of adjustments, condenser and diaphragm - Change of objectives - Optical sections - Care of microscope . . . . . . . pages $\mathrm{I}-8$

\section{LECTURE II.}

\section{Structure of a Cell.}

Physical and chemical nature of the cell-wall-Physical properties of protoplasm-Diffusion-Permeable and semi-permeable partitions-Osmotic pressure-Turgor-Plasmolysis-Coagulation-Chemical nature of the constituents of protoplasm . . . . . . pages 9-18

\section{LECTURE III.}

\section{SACCHAROMYCES CEREVISEAE.}

Crystalloidal and colloidal states-Size and surface of colloidal particlesSols and gels-The yeast plant-A single cell-Growth and reproduction-Temperature limits-Assimilation pages $19-27$

\section{LECTURE IV.}

\section{SACCHAROMYCES CEREVISEAE.}

Changes facilitated by enzymes-Nature of enzyme action-InversionEnergy needed for assimilation supplied by combustion-Fermentation, the incomplete combustion of carbohydrates-Anaerobic respirationSize of yeast-cells-The ghost-micrometer . . . pages 28-34

\section{LECTURE V. \\ Chlamydomonas.}

Size and motion of the cell-Its structure-Stimulus and response-Spontaneity-Sexual and asexual reproduction-Gametes and spores 
LECTURE VI.

Chla mydomonas.

Formation of carbohydrates in Chlamydomonas-Source of carbon-Photosynthesis-The pigments of the chloroplast-Relation of green plants to solar energy and to the rest of living nature-Movements of protoplasm pages $4 \mathrm{r} \cdot 4^{8}$

\section{LECTURE VII.}

\section{BACTERIA.}

Energy set free by aerobic respiration of Chlamydomonas-Catabolism and anabolism-Elimination of waste-Contractile vacuoles-Form, size and motion of bacteria - Reproduction-Resistance to high temperatures-A smear-preparation . . . . . . pages 49-56

\section{LECTURE VIII.}

\section{BACTERIA.}

Constituents of bacteria-Spontaneous generation-Methods of sterilisation - Pure cultures-Sources of food for bacteria-Respiration of bacteriaNitrifying and sulphur bacteria . . . . pages 57-64

\section{LECTURE IX.}

\section{SPIROGYRA PORTICALIS.}

Circulation of nitrogen-Cells of Spirogyra-Microscopic reconstructionAsexual and sexual reproduction-Mechanism of conjugation

pages $65-74$

\section{LECTURE X.}

\section{VOLVOX AUREUS.}

Unicellular and multicellular plants-Structure of Volvox-Its motion and nutrition-Asexual and sexual reproduction-Differentiation of sexual cells-Sperms and ova-Development of embryos-Somatic and germ cells

\section{LECTURE XI.}

VAUCHERIA SESSILIS.

Structure of Vaucheria - A cœnocyte-Division of labour-GrowthAsexual reproduction - Zoospores - Sexual reproduction-Antheridia and oogonia.

\section{LECTURE XII.}

\section{MUCOR.}

Appearance and structure of white mould-Chemotaxis-Response to gravity and to light-Formation of sporangium and spores-Germination of spores-Sexual reproduction-Nutrition and respiration of Mucor 


\section{Contents.}

\section{LECTURE XIII.}

\section{PENICILLIUM GLAUCUM.}

Mycelium of blue mould-Conidiophores and conidia-Origin of ascospores -Enzymes of Penicillium - Action on tartaric acid-Respiratory ratio pages $97-\mathrm{ro3}$

\section{LECTURE XIV.}

\section{FUCUS PLATYCARPUS.}

General appearance and histology of Fucus-Apical growth-Its sources of food-Its reproductive cells-Antheridia and oogonia-Origin of sexual reproduction - Fertilisation-The nucleus contains the germ-plasm

pages 104-II2

\section{LECTURE XV.}

POLYSIPHONIA FASTIGIATA.

Structure of Polysiphonia-Microscopic reconstruction-Apical growthProtoplasmic connections-Manner of nutrition-Life-history-Origin of tetraspores-Germination-Sexual plants-Antheridia and procarpsOvum or carpogonium-Fertilisation-Auxiliary cell-CarposporesRelation of tetrasporic to carposporic plants-Alternation of generations pages II 3 - $\mathbf{1 2 0}$

\section{LECTURE XVI.}

\section{Marchantia PoLYmorpha.}

Origin of land-plants-External appearance of plants of Marchantia-Minute structure of the thallus - Connection between death and reproductionReproduction by means of gemmæ . . . . . pages I2I-127

\section{LECTURE XVII.}

\section{Marchantia PoLymorpha.}

Sexual reproduction-Male and female plants-Antheridiophore-Liberation of sperms-Archegoniophore-Passage of sperms to archegoniaOosperm - Development of embryo - Spore-capsule - Liberation and germination of spores-Life-history-Gametophyte and sporophyte

\section{LECTURE XVIII.}

\section{FUNARIA HYGROMETRICA.}

Protonema, leafy plant and sporangium-Male and female branchesAntheridia and archegonia-Fertilisation-Development of oospermTheca-Calyptra-Shedding of spores - Sporophyte and gametophyteComparison of alternation of generations in Polysiphonia, Marchantia and Funaria . . . • • . • • pages $\mathrm{I}_{35} \mathrm{I}_{45}$ 


\section{LECTURE XIX.}

\section{ASPIDIUM FILIX-MAS.}

The "male fern"-Formation of leaves-Arrangement of the conducting tracts in the fundamental tissue of the stem-Branches supplying roots and leaves-Structure of the conducting tracts-Structure of roots and leaves-Disposition and protection of photosynthetic cells-StomataPath of water from roots to leaves-Transpiration current-Rigidity of land-plants

\section{LECTURE XX. \\ ASPIDIUM FILIX-MAS.}

Life-history of Aspidium-Sporophylls-Sori-Sporangia-Spores-Mechanism for disseminating spores-Germination of spores-GametophyteAntheridia and archegonia-Fertilisation aquatic-Embryo growing from oosperm becomes sporophyte-Amphibious life-history-Archegoniates-Homologues

- pages $\mathrm{x} 6 \mathrm{I}-\mathrm{I} 69$

LECTURE XXI.

SELAGINELLA MARTENSII.

Synthetic forms-Stems, leaves, rhizophores and roots of Selaginella-Cones of sporophylls-Microspores and megaspores-Heterospory-Male and female gametophyte-Diminutive dependent gametophytes-Fertilisation -Embryo enclosed in female gametophyte still partly enclosed in sporecoat

\section{LECTURE XXII.}

\section{PINUS SILVESTRIS.}

Pine tree-Arrangement of scales and dwarf shoots on its branches-Protective, photosynthetic and conducting tissues of the leaves-Connection of leaves with stem-Structure of stem-Its growing tip-Growth of stem in thickness-Root--Direct connection between absorbing ends of roots with transpiring leaves . . . . . . pages I79-IgI

\section{LECTURE XXIII. \\ PINUS SILVESTRIS.}

Development of a tracheid-Bordered pits-Comparison of transverse, radial and tangential sections in the wood-Outer tissues of the stem-Possible functions-Pollen-cones-Stamens, sporophylls-Pollen-sacs, sporangia -Pollen-grains, spores . . . . pages r92-203

\section{LECTURE XXIV.}

PINUS SILVESTRIS.

Seed-cones - Carpels, sporophylls - Ovules, megasporangia-Embryo-sac, megaspore-Pollination-Growth of the male and female gametophyte - Sperms and ova-Embryo-Seed-Comparison of the life-history of the Pine with that of the Archegoniates . pages 204-2I4 


\section{LECTURE XXV. \\ RANUNCULUS BULBOSUS.}

Gymnosperms and Angiosperms - The buttercup-Structure of the stem and its conducting tracts-Function of the bundle-sheath-Arrangement of tissues in the leaves and roots-Cohesion-theory of the ascent of water in plants-Meaning of the subdivision of the water channels and of the pits-Root-pressure

\section{LECTURE XXVI. \\ RANUNCULUS BULBOSUS.}

Flowers of the buttercup-Stamens and carpels--Origin of the pollen-grains and embryo-sacs - Liberation of pollen-grains - Insect-pollinationGrowth of male gametophyte-Development of female gametophyteFertilisation-Embryo and endosperm-Seed-Comparison with the Pine pages $230-238$

\section{LECTURE XXVII.}

SCILLA NUTANS.

Dicotyledons and Monocotyledons-The wild hyacinth-Arrangement of tissues in roots, stems and leaves-Stamens-Fused carpels-Gametophytes-Embryology

- pages 239-249

\section{LECTURE XXVIII.}

\section{Nuclear Division.}

All cells of adult directly descended from oosperm-Parts of a nucleusSomatic mitosis-Chromomeres of nuclei in adult derived by fission from those of oosperm-Chromatin : the germ-plasm-Reducing mitosis -Want of equivalence in the chromomeres of resulting nuclei

pages 250-259

\section{LECTURE XXIX.}

HEREDITY.

Inherited and acquired characters-Mendel's observations-DeterminantsDominance-Proportions of progeny showing the dominant and the recessive character - Segregation of determinants - Interaction of characters

\section{LECTURE XXX. \\ Evolution-Theory OF DEscent.}

Origin of species by evolution-Evidence from morphology, from distribution and from palæontology-Natural selection-Lamarkism-Segregation and new combinations of determinants . . pages $270-278^{\circ}$ 



\section{LECTURE I.}

IT is convenient to begin the study of plants with the simplest. The most simple are as a rule the smallest.

To make out the structure of these very small plants the use of a microscope is essential. Hence we commence our course by learning how to use a microscope: Here on the screen is a figure which will give you the names of the different parts of a microscope.

Set the instrument on a table opposite a window, preferably one facing the north. The pillar of the microscope should be placed next the observer. Turn the mirror so that the light from the sky is reflected upwards from the plane surface of the mirror through the aperture in the stage up the body of the microscope. See that the iris-diaphragm below the condenser is open and that the low objective (or power) is in position at the lower end of the tube (or body). With these preliminary arrangements made, it will be well to apply the microscope to some suitable object and learn something of how it may be used to show minute structure. It must be understood that, as the microscope extends our powers of vision, so as practically to endow us with a new sense, this new sense, like our other senses, requires training and cultivation, and it is only after considerable practice that we can interpret truly the sensations conveyed to us by this instrument.

A very suitable object for the beginning of this training is furnished by the skin of a leaf. We will select the leaf of Tradescantia virginiana (Spiderwort). To obtain a preparation of this skin, proceed as follows: take the leaf, which is long and narrow, and bend it across the fore finger of the left hand with the upper, or grooved, side outwards. With a sharp knife, or razor, nick the surface of the leaf without cutting deeply into it. Raise a little flap of skin, the leaf-skin, and catch it with a forceps. You will in this way easily peel off a portion of the membranous skin. Lay the piece of skin so obtained in a drop of water on a glass slide. The smooth upper side of the skin should be laid uppermost in the drop. Clean a cover-glass like this one I hold 
here, and means of a forceps lay it on the drop containing the piece of skin.

In cleaning the cover-glass, gently hold it by the edges with the finger and thumb of the left hand, while the finger and thumb

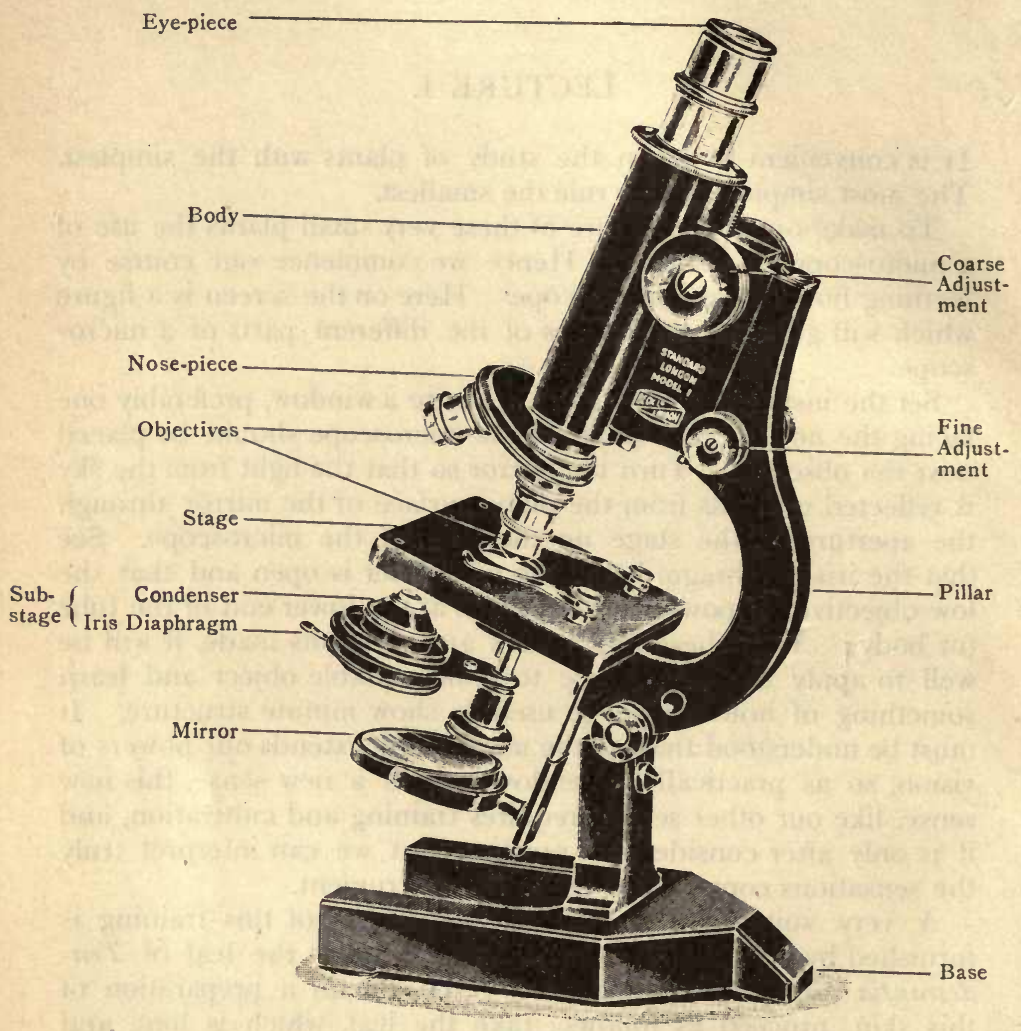

FIG. I.-The microscope.

of the right hand, covered with a fold of fine linen, may rub it clean, simultaneously pressing from both sides.

The piece of skin thus enclosed in a drop of water between the slide and cover-glass is a preparation ready for microscopic examination. Very probably a large air-bubble will be found in the preparation. This may be no disadvantage, if it is not in contact 
with the object, but on the other hand it may cause inconvenience if it adheres to its surface.

The bubble has become enclosed because the outer surface of the piece of skin acts as if it were greasy and is not wetted by the water which is slightly raised up round the object. When the cover-glass is put on, the air in the hollow immediately over the piece of skin is trapped. If before the cover-glass is applied the piece of skin is wetted by stroking it with a camel's hair brush or a needle, and the cover-glass then let down slightly obliquely so that one edge touches the water first, no air will be enclosed in the preparation.

The preparation should now be inspected to see that there is no water on the under side of the slide, and no water or dust on the upper side of the cover-glass. Water on the slide may be carefully dried off, but if the cover-glass is not perfectly clean it must be taken off again (raising it with a needle or point of a forceps), carefully polished and replaced.

'The preparation thus made is now ready for examination. Place it on the stage of the microscope. I will assume that the microscope is provided with two objectives (powers): one (the low power) focussing about $\mathrm{I} \mathrm{cm}$., and the other (the high power) focussing I or $2 \mathrm{~mm}$. above the object. Set the front lens of the low power about I $\mathrm{cm}$. above the cover-glass by means of the coarse adjustment. Look into the eye-piece and turn the mirror until the eye-piece appears filled with light. Close the diaphragm below the condenser till its opening is about $\frac{1}{8}$ of the total aperture. Turn the coarse adjustment until the object comes into focus. Now turn the fine adjustment until you find the position in which the object appears with greatest distinctness. It is usually best to use the flat surface of the mirror when the condenser is in position, but sometimes with this arrangement extraneous objects such as the woodwork of windows are focussed on the object and become inconvenient. This inconvenience may be removed by using the concave surface of the mirror, or by altering the level of the condenser.

When sharp focus has been obtained you will see that the piece of leaf-skin is divided up into small and fairly uniformly sized areas. They are nearly rectilinear in outline, and fit closely against one another like tiles in a pavement. These areas correspond to the outlines of cells-the units of which the skin and all other tissues are built. A darkish rounded body may be seen in most of these cells. It is called the nucleus. While making these observations the inclination of the mirror should be 
repeatedly changed and the effect of these changes observed; the aperture of the diaphragm should be altered and the changes in appearance so caused studied. It will be found that while a closed diaphragm causes the outline of a cell to appear dark, this outline becomes brilliant when the diaphragm is opened. Similar changes may be observed in the nucleus. By nearly closing the diaphragm you will be further able to perceive that the cells are not absolutely clear but are more or less shaded by the presence of a haziness especially near their boundaries and round the nucleus. This

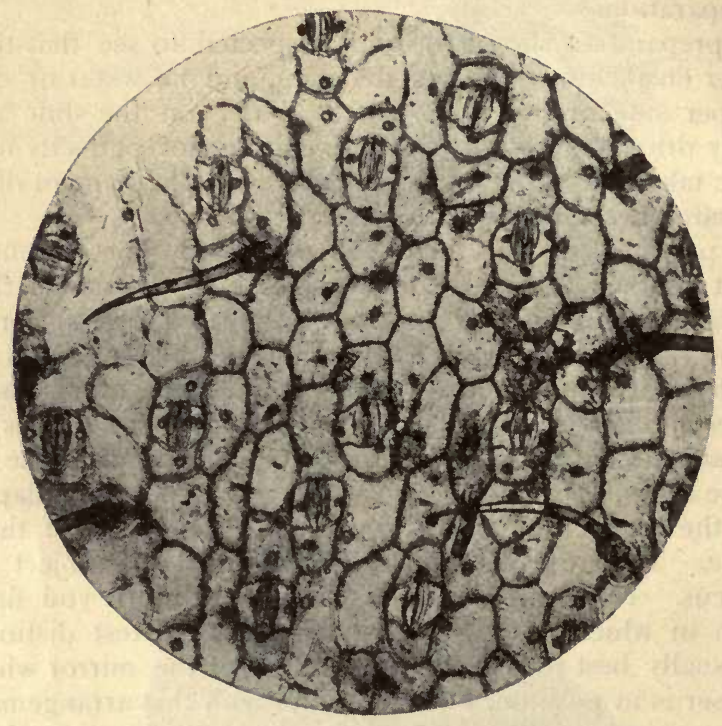

FIG. 2.-Tradescantia virginiana, lower epidermis, microphotograph $\times 100$.

hazy substance together with the nucleus is the protoplasm of the cell. The use of the high power (or objective) will show the parts of the cells more clearly. It is necessary to use great care when changing from the low to the high objective. It is well first to assure oneself that the cover-glass is quite clean and that there is no water on its upper surface; after this, carefully set the cell selected for special examination exactly in the centre of the field. With microscopes of the best construction it is only necessary to turn the nose-piece round bringing the high power 
into position under the body of the instrument, then the accurate construction of the nose-piece and objectives secures that the high power is exactly over the centre of the field previously viewed by the low power and the object will be approximately in focus for the high objective.

Such exactitude of construction is not always to be found in microscopes, so that certain precautions must be taken in order to make sure to avoid injuring the high power, and spoiling the preparation by dragging the lens of the objective against the coverglass. If by any chance the front lens should get smeared it should be immediately cleaned carefully with a piece of clean linen. In order to avoid any mishaps in turning on the high objective you should proceed as follows: Raise the body of the microscope by turning back the milled head of the coarse adjustment about one quarter revolution then turn round the nose-piece bringing the high power into position, and almost completely close the diaphragm. The front lens should now be about $3-5 \mathrm{~mm}$. above the cover-glass. Put your eye to the eye-piece and while looking down the now somewhat darkened tube rack the coarse adjustment very slowly and carefully downwards. Soon hazy objects will loom up in the field. Now cease using the coarse adjustment and transfer your hand to the fine adjustment ; by moving this you will quickly bring one of the cells into sharp focus. When it is in focus carefully note the distance of the front lens from the coverglass by viewing it from the side. In order to be able to make the change from low to high objective more speedily in future, it is well now, without altering the focus, to switch back the low power and note if it is still in focus ; or, if adjustment is required, note if it is necessary to raise or lower the body. If no adjustment is necessary, or if the tube has to be raised to bring the low objective into focus, then you will know that when making the change from the low to the high power in future you need not screw back the coarse adjustment but simply switch on the high objective after ascertaining the cleanliness of the cover-glass. If, when turning back from the high objective, the low objective requires to be depressed to bring it into focus, then you must always raise the coarse adjustment before switching on the high power.

When the high power is put into position one should try many experiments with the mirror and the diaphragm in order to obtain the most satisfactory combination of arrangements for the study of each object. Above all, special attention must be given to the fine adjustment. The high power has a very slight depth of focus. That is, when it is in focus for any one minute object, objects 
very slightly nearer or further are out of focus and appear blurred. Hence when we look at a microscopic field with a high power only those objects which are at a certain level in the preparation are distinctly visible to us, and in order to appreciate the relation of the various objects in the preparation to one another we must continually keep shifting the focus up and down. Thus while using the high power it is advisable to keep one's finger on the fine adjustment and to move its milled head continually backwards and forwards.

While moving the focus for the high power it becomes apparent that each cell is bounded by a transparent membrane-the cell-wall. As we look down on the cell its outline is generally hexagonal or pentagonal. One can see that the wall is colourless and that the light from the mirror passes through it. It is transparent. When the focus is at a certain level the thickness of the wall is seen in section. By carefully focussing upwards with the fine adjustment one can see that the wall is continuous across the top of the cell next the cover-glass, and with skilful manipulation of the mirror and diaphragm one can see that the outer surface is finely corrugated. In the same way the cell is closed below. The cell, in fact, at which we are looking is a flat hexagonal or pentagonal box. The walls of this box are made of a transparent material. Looking through the transparent top we can see the contents of the box illuminated by the light transmitted through the equally transparent bottom.

The most easily observed object within this cell is the nucleus. Its position in the cell appears to be fortuitous. It is dark or bright according as the diaphragm is closed or open. It is colourless. At first sight it appears to be disc-shaped, but when very careful focussing is used it is possible to see that it is approximately globular in form; it appears disc-shaped because only one level or section of it appears in focus at one time. By focussing deeper and deeper one can compare a series of these focal levels, or optical sections, with one another and mentally reconstruct from them the shape of the whole. It is evident that the optical sections of an approximately spherical object are a series of more or less circular discs. The nucleus is then a spherical or globular body.

During these observations another substance within the cell will be forcing itself on our attention. Round the nucleus and adhering to the inside of the cell-wall is a colourless and slimelike material. When the diaphragm is closed this material is lighter than the nucleus, when the diaphragm is open it is less 
bright, and with this arrangement it is very indistinct and hard to make out. This material, together with the nucleus, forms the protoplasm of the cell. It forms an irregular lining to the cellwall. In places the lining is thick and in places again it is drawn out to an infinitesimally thin pellicle. Embedded in the protoplasm are various smaller or larger specks which are often called granules. In the cells before us, as is often the case in cells composing tissues which have ceased to grow, the protoplasm occupies but a small proportion of the space enclosed by the wall. The greater part of the cavity of the cell is occupied by a drop of fluid called the vacuole. Sometimes strands of protoplasm may be seen striking across it or even it may be broken up into a number of small vacuoles separated from one another by sheets or partitions made of the slimy protoplasm. Often some of the granules seen in the protoplasm are minute vacuoles.

\section{PRACTICAL WORK.}

Acquaint yourself with relations of the various parts of the microscope and learn their names.

Mount a piece of the leaf-skin of Tradescantia virginiana in a drop of water. Cover with a clean cover-glass.

Put the low power in position and set the preparation on the stage.

Adjust the mirror so that light from the window is concentrated by the condenser on the object.

Focus the object and try how changes in the mirror, the diaphragm and the level of the condenser alter the microscopic appearance of the object.

Count the number of cells on the diameter of the field.

Note the outlines and the nuclei of the cells.

Adjust the middle of the fragment of skin to coincide with the centre of the field.

Raise the body of the microscope by means of the coarse adjustment. Switch on the high power.

By means of the coarse adjustment bring the front of the high power to about $4-5 \mathrm{~mm}$. from the cover-glass.

Close the diaphragm to a small aperture.

With your eye constantly looking into the eye-piece rack the body of the microscope very slowly downwards. As soon as dark indistinct objects begin to loom into view transfer your fingers to the fine adjustment, and by turning it downwards bring the cells into focus. Now observe the distance of the high power from the cover. When the focus has thus been obtained switch back the low power and note if it is necessary to raise or depress the body to bring it into focus.

Again count the number of cells occupying the diameter of the field. This will give an idea of the relative magnifications of the high and low powers. Try the effects of various adjustments of mirror, diaphragm and condenser. Focus for the near side and the far side of the nucleus and for its diameter.

Carefully draw an optical section of a cell in the plane of its nucleus.

Make your sketch on smooth paper with a sharp pencil. Your sketch 
should show the outline of the cell, the thickness of its vertical walls, the limits of the protoplasm and vacuoles, the nucleus and some granules.

Replace the low power in position ard take the slide from the stage.

Draw off some of the water between the cover-glass and slide by means of a piece of blotting-paper, and add a drop of liquor iodi (a solution of iodine in a watery solution of potassium iodide) at the opposite side of the coverglass.

Note how the iodine passes through the cell-wall and stains the protoplasm, especially the nucleus.

Before leaving your microscope return the low power to position under the body and remove the preparation.

Never remove a preparation, or place it on the stage while the high power is in position. Leave the diaphragm open and the plane mirror facing upwards. See that all parts of the microscope are clean and dry. 
From the few observations already made some idea may be formed of the different parts of the cells taken from the leaf-skin. It is difficult, however, to realise immediately the physical nature of these different microscopic objects. All tissues of plants are made up of cells resembling, more or less closely, those we have examined in the skin of the leaf of Tradescantia virginiana, so that if we can obtain clear ideas as to the nature of the parts of these cells we can apply them with little modification to all others.

It is easy to see the nucleus and the rest of the protoplasm when looking through the walls of the cells taken from the skin of the leaf. The substance forming the wall is therefore evidently transparent; and it is also without colour. One can see, however, the surface of the cell-wall and it is also quite easy to distinguish it from the surrounding water. We distinguish it just as we distinguish a piece of glass in air or in water, not necessarily by its different colour, but because it bends the rays of light differently from the surrounding air or water. It has, in fact, a different refractive index. The substance forming the cell-wall is then transparent, colourless and has a higher refractive index than water. It is owing to these physical properties that it looks bright when illuminated with an open diaphragm and dark when the diaphragm is closed.

The torn edge of the preparation is formed by partially empty cell-walls. By pressing on the cover-glass with a needle it is easy to distort these pieces of cell-wall. When the pressure is relieved they recover their former shape. The cell-wall evidently possesses a certain amount of rigidity and is elastic. It also possesses tenacity and is tough. This will be realised when one remembers that a sensible pull had to be exerted in tearing the piece of skin and that the strip torn off from it is tough and can sustain a considerable pull. It is also to be remembered that the actual cross section of cell-wall sustaining this pull is very small; for but a very small portion of the strip is composed of the walls. The actual strength of the cell-wall compared with other substances 
can be easily measured by using the walls of other cells more suitable for the purpose. Thus the fibres of cotton-wool are the walls of very long narrow cells in which the internal cavity of the cell has been almost obliterated by the thickening of the wall. It is possible by measuring the weight, which one of these fibres will sustain, and also its own cross section, to determine directly the tenacity of its substance. It has been found that the breaking weight of this material is about $4^{\cdot 2}$ tonnes per sq. $\mathrm{cm}$. This is about the same tenacity as that of wrought iron.

Our preliminary observations have brought to light yet another very important property of this material. When the liquor iodi was applied to the outside of the cell-wall, it quickly diffused through the wall and coloured the nucleus within. Evidently the cell-wall is permeable to this substance dissolved in water. It is in fact permeable to all substances dissolved in water.

Chemically the substance forming the cell-wall is known as cellulose. It is a carbohydrate, i.e. a compound containing carbon, hydrogen and oxygen, in the molecule of which there are two hydrogen atoms to each oxygen atom. It has a large and complicated molecule, having the formula $\left(\mathrm{C}_{6} \mathrm{H}_{10} \mathrm{O}_{5}\right)_{n}$.

The protoplasm enclosed by the cell-wall is much less conspicuous than the wall itself; but this does not indicate by any means that it is of less importance than the wall. Indeed, as will appear later, it forms the essential part of the cell.

By similar observations to those made on the cell-wall, we can easily ascertain that protoplasm is colourless, transparent and of a higher refractive index than water; but in this cell its refractive index is not so high as that of the cell-wall. Where the wall of a cell is broken the protoplasm emerges, and it may be seen to change shape slowly, like a sticky, viscid liquid. It adheres to the fragments of the cell-walls and it shows no rigidity or elasticity like cellulose. In fact, it behaves very much like the white of an egg, and in the cell we are examining the protoplasm forms a sticky, viscid lining to the cell-wall, which might be compared to a smear of white of egg on the inner surface of a transparent box. In this viscid film are often embedded minute granules; sometimes again the granules are absent, and the protoplasm seems homogeneous or almost so. By closing the diaphragm it is generally possible to make out a definite surface between the protoplasm and the water in the vacuole.

The protoplasmic lining of the cell-wall encloses the liquid of the vacuole. This liquid is a watery solution of various substances. Unlike the cell-wall the protoplasm obstructs the passage 
of dissolved substances. This may be readily seen in cells where a coloured substance is in solution in the vacuole. We often find such coloured cells in the skin of 'Tradescantia; but it is more convenient to observe this effect in a tissue in which all the cells are coloured, such, for example, as the fleshy part of the beetroot, or the petal of a rhododendron. If a piece of one of these tissues is mounted in water it may be observed that as long as the protoplasm is uninjured it confines the dissolved red pigment within the vacuole and does not allow it to escape into the surrounding water.

This property of protoplasm of obstructing the passage of solutes (substances in solution) is one of very great biological importance and must be considered shortly in relation to the nature of diffusion and of solution.

When a crystal of a soluble substance, e.g. any soluble salt or sugar, is placed in water it dissolves, and the dissolved substance slowly spreads itself throughout the surrounding water. This spreading or diffusion, as it is technically called, takes place quite apart from the mingling of the solution with water due to currents, and even when the crystal lies in the bottom of a vessel, diffusion may be seen to overcome gravity and in time to secure the uniform distribution of the solute throughout the water.

A mental picture of diffusion may be formed by supposing that in solution the bonds that bind the particles of the solute to one another are broken, and they, by virtue of the energy they possess, spring forth into the solvent: here they are free to move in the direction of their first spring till they encounter some other particle or the walls of the vessel. At each collision they rebound only to be hurled back again when they strike another obstacle. In the solution of the crystal we must imagine countless millions of these particles launched, jostling each other, rebounding from the impacts, and thus gradually, with a disorderly and turbulent swarming, tending to distribute themselves uniformly at last throughout the liquid. Thus regarded the particles of a dissolved substance behave in a manner very similar to those of matter in a gaseous state.

The process of diffusion is seen in action in these two tall glass cylinders of water into which a few crystals of potassium bichromate and copper sulphate have been placed respectively. The crystals which were put in a few days ago have almost disappeared and a dense solution of each salt is formed in the bottom of each jar. If you look carefully you will see that there is no definite surface for the solutions, but a gradual transition from the densely 
coloured solution below to the colourless water above. As the cylinders are observed day by day this zone of gradation will be seen to rise higher and higher, until finally the solutes are equably distributed throughout the liquid. Under ordinary conditions, no doubt, currents hasten this uniform distribution but even when these are eliminated, diffusion is adequate, given time, to accomplish it alone.

Evidently if we could interpose a partition between the solution and the water above, and if this partition obstructed the passage of the solute while it allowed water to permeate it, the diffusing particles, as they spread through the water would be checked by the partition. As they dash forward they will strike the membrane and will be reflected from it into the surging mass of their fellows below: rebounding from these they are again hurled against the membrane. The sum of the myriad impacts of this intense bombardment will act as a pressure pushing back the partition.

Partitions of this nature, which obstruct the passage of dissolved substances while they allow the solvent to pass freely, are well known. They are found in nature and they may be artificially made. Ordinary animal and vegetable parchments act more or less completely in this way, and by their means the pressure produced by a diffusing solute may be easily seen.

One of the simplest ways of observing this is as follows: the open ends of a piece of parchment tube, such as is used by chemists for dialysis, are each bound on to a tightly fitting rubber stopper. One of the stoppers is perforated and supplied with a glass or ebonite stop-cock. Thus fitted, the tube is filled with a strong solution (20 per cent.) of sugar. The stop-cock is closed and the whole is submerged in clean water. After a short time the parchment tube will be found distended and bulged owing to the diffusion of the sugar particles pushing out the parchment. Soon the pressure begins to stretch the parchment and if we open the stop-cock the elastic recovery of the parchment will cause a jet of the solution to issue from the cock, giving a visible demonstration of the pressure.

A dried raisin put into water is noticed to swell and ultimately become distended owing to the same phenomenon. Water passes through its skin, and the sugar and other soluble substances within go into solution and tend to diffuse or spread in the water. In this, however, they are opposed by the obstruction offered by the skin to their passage, and the pressure developed by the diffusion of the dissolved particles pushes the flaccid skin out- 
wards in all directions until it takes on a globular form and becomes tightly stretched.

Another simple experiment shows that the pressures generated in this way are very considerable: Here is a thistle funnel ; across its opening I have fixed a piece of parchment. To prevent leakage the edge of the funnel was smeared with glue and while the glue was soft the parchment was bound tightly on with string. When the glue was set the funnel was inverted and filled with a sugar solution and immersed in water, while the tube of the funnel was supported in a vertical position projecting above the water. After a short time it could be noticed that the liquid in the funnel tube was rising and it soon mounted above the level of the surrounding water. This rise may be ascribed to the pressure of the diffusing sugar particles expanding the confines of the sugar solution, and the process may be described in terms of the hypothesis which attributes the pressure to the bombardment of the dissolved particles. The parchment and glass of the funnel being fixed, resist this pressure, but the particles hurling themselves from below against the surface of the solution in the tube and being unable to escape from it, press it upwards and so make room for more water to enter through the parchment into the tube. Thus we may regard the weight of the column of liquid as being raised above the surrounding water by the bombardment of the dissolved sugar particles. After a time, the solution will have risen the full length of

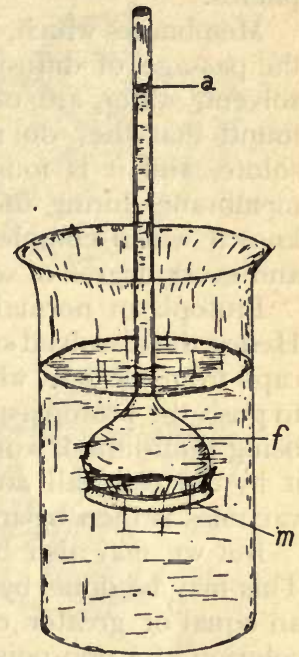

FIG. 3.-Glass funnel $(f)$ closed below with a semi-permeable membrane $(m)$. In $f$ is a solution, the upper surface of which is seen at $a$. The beaker contains water. the funnel tube, and, if we wish to continue the observations, we must add to its length. The height of the column of liquid thus raised may be very great. Its rise will only cease when the diffusion-pressure of the solution-or osmotic pressure as it is usually called - is balanced by this hydrostatic head.

By careful measurements it has been found that the height to which the osmotic pressure can raise a column of solution depends upon the concentration of the solution: in other words, the osmotic pressure of a solution is proportional to the number of 
dissolved particles there are in unit volume. Measurement has also shown that the osmotic pressure is directly influenced by temperature, so that the osmotic pressure of dissolved particles is very similar to the pressure produced by gas particles, and obeys similar laws. At ordinary temperatures the osmotic pressure of a 5 per cent. solution of cane sugar is about 3 atmospheres, and that of a 5 per cent. solution of common salt is about 40 atmospheres.

Membranes which, like those used in these experiments, obstruct the passage of diffusing solutes while they are permeable to the solvent, water, are called semi-permeable. In many cases it is found that they do not absolutely preclude the passage of the solute, and it is found that some sugar passes out through the membrane during the observations. But other membranes are known which completely obstruct the passage of certain solutes and every degree of semi-permeability may be met with.

Protoplasm normally exhibits a very perfect semi-permeability. Hence the dissolved substances in the vacuoles of cells cannot escape from the cells when they are surrounded with water but tend to push the protoplasm out against the cell-wall. The protoplasm being semi-liquid, would flow before the pressure but is held up to it by the cell-wall and the osmotic pressure of the solutes of the vacuoles is then balanced by the tension of the cell-wall.

But we may also balance it by an external osmotic pressure. This may be done by immersing the tissue in a solution having an equal or greater concentration than that in the vacuole. A solution of a non-poisonous substance should be used. If a thin slice of beetroot, or thin flap torn off a rhododendron petal is mounted in a drop of a ro per cent. solution of common salt, you will see, as you observe it in the microscope, that the rose-coloured vacuoles, which at first entirely filled the cavities of the cellssave for the space occupied by the delicate containing film of protoplasm-are now contracting into small densely coloured globules in the centre of the cell cavity. The explanation of this contraction is as follows: The external salt solution has diffused through the permeable cell-walls and come into contact with the film of protoplasm. The diffusing particles not being able to penetrate the protoplasm push against it and, being more concentrated than the dissolved particles in the vacuole, exercise a superior pressure and drive the film away from the wall into the cavity of the cell. A cell in this state is said to be plasmolysed. It is evident that while the external osmotic pressure is thus driving in the protoplasm it reduces the size of the vacuole, and 
as the internal solutes cannot escape their particles become more crowded and concentrated. At a certain stage the reduction of volume of the vacuole brings the concentration of the solutes in the vacuole to be the same, reckoned in dissolved particles, as that of the external solution. This increase of concentration is made apparent in the examples we have taken by the intensification of the red colour of the vacuole; for the dissolved pigment like the other solutes has been concentrated and hence its colour deepens proportionally.

If a tissue whose cells are thus in a state of plasmolysis is transferred from the salt solution into water, very soon the osmotic pressure of the solutes in the

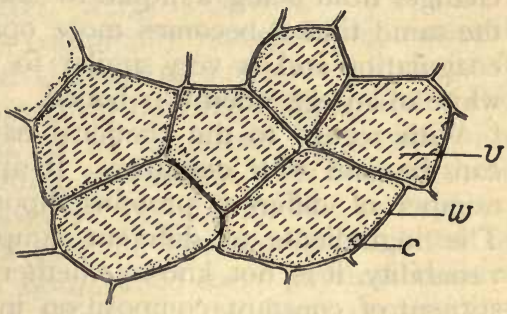
vacuoles, no longer opposed by an external osmotic pressure, pushes the protoplasm outwards and presses it again against the cell-wall and distends the latter.

These observations show that a cell which is abundantly supplied with water will normally have its cellwall distended by osmotic pressure. In this distended state, which is called turgor, it is rigid. One may com-

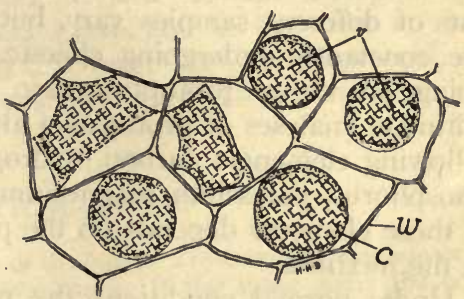

Fig. 4-Cells of rhododendron petal, $x$ ro3. $c$, cytoplasmic lining of cell; $v$, vacuole; $w$, cell-wall. Below the same cells are seen plasmolysed. pare the rigidity of a football or pneumatic tyre-the bladder or the inner tube playing the part of the protoplasm, while the leather or the cover is put into a state of tension like the cell-wall.

The compressed air cannot penetrate the bladder, just in the same way as the dissolved particles cannot pass through the protoplasm, while the leather cover in one instance and the cellwall in the other takes up the pressure and prevents the inner envelope extending indefinitely. The impermeability of the inner envelope is necessary in each case and any injury to it which would render it permeable will quickly destroy rigidity. A temperature of about $70^{\circ} \mathrm{C}$. changes protoplasm profoundly, and among other changes taking place at this temperature, it will be 
found that it becomes permeable to dissolved substances. Thus if cells containing a coloured solution in their vacuoles be heated to $70^{\circ} \mathrm{C}$. the coloured fluid permeates the envelope and escapes. At the same time the tissue which had been turgid and stiff becomes limp and flaccid, as the osmotic pressure of its component cells is relieved.

At the temperature of about $70^{\circ} \mathrm{C}$. protoplasm sets, i.e. it changes from being a liquid to take on a semi-solid state. At the same time it becomes more opaque. This change is called coagulation and is very similar to the change undergone by the white of an egg when it is boiled.

With regard to the chemical nature of protoplasm very little can be said with certainty. It appears to be a mixture of a number of different proteins, lipoids, carbohydrates and salts. The ingredients of different samples vary, and, owing to this variability, it is not known whether there is some essential constituent of constant composition in which the other compounds are embedded in varying proportions. Not only do the ingredients of different samples vary, but within the same sample they are constantly undergoing chemical change. These interactions going on within protoplasm are spoken of as its metabolism. Ultimate analyses of protoplasm always show the presence of the following elements : carbon, hydrogen, oxygen, nitrogen, sulphur, phosphorus, potassium, magnesium and calcium. The proportion of these elements depends on the proportions of their compounds in the mixture.

Under normal conditions the protoplasm of cells contains a very large proportion of water: 80 per cent. by weight, or more, is usually present. Proteins rank second by weight, and it is probably these substances which confer on protoplasm its unique properties. These bodies may be defined as nitrogenous substances, which on hydrolysis break up into amino-acids, i.e. organic acids characterised by containing one or more aminogroups $\left(-\mathrm{NH}_{2}\right)$ and one or more carboxyl groups $(-\mathrm{COOH})$. These groups confer on the amino-acids basic or acid qualities depending on the predominance of either group. Consequently they may behave as weak bases or acids. The amino-acids also may combine mutually by the elimination of water from the uniting carboxyi and amino-groups e.g. :-

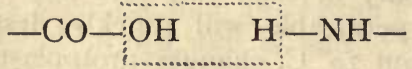

In this way many amino-acids may link together and bodies with 
a very large molecular weight may be built up. The formation and dissolution of this linkage, which is called the peptide linkage, possibly indicates one of the frequent changes occurring in the metabolism of protoplasm.

The other substances such as lipoids (fat-like bodies), carbohydrates and salts are probably present in the protoplasm not as essential constituents but as stores from which the much more complex amino-acids and proteins are built up and renewed, and from which, as we shall see later, energy is derived.

The protoplasm of cells is continually incorporating external materials in its substance and eliminating substances from itself into its surroundings. It is constantly building itself up and at the same time disintegrating, and, as we have seen, the compounds within its own substance continue to interact. As long as these changes continue the protoplasm is "alive". In fact, protoplasm is the living part of the cell and the phenomena exhibited by protoplasm are life. It has been called the "physical basis of life". When protoplasm is killed, e.g. by heat, constructive metabolism comes to an end. With certain exceptions its external and internal reactions cease, and it becomes an inert mass of proteins, etc. It is in this form often called proteid, and as we have seen it both loses its liquid nature and becomes permeable.

\section{PRACTICAL WORK.}

Mount a small piece of the skin of the leaf of Tradescantia virginiana in a small drop of water. Put on a cover-glass. If the space under the coverglass is filled with the drop draw off a little water by applying a piece of blotting-paper to the $\epsilon$ dge of the cover. Now apply a drop of liquor iodi and let it run in under the cover. See that the upper surface of the cover-glass is quite clean and place the slide on the stage of the microscope. Observe how the cells of the tissue change in appearance, and those first which are at the edge of the specimen. The protoplasm takes on a brownish hue and minute granules become apparent in it. The nucleus stands out clearly. The iodine in solution has permeated the cell-walls and brought about these changes within the cell.

Mount a thin section of beetroot, or a thin flap cut from the crimson petal of a rhododendron flower, in a small drop of water. Make a sketch, using the high power, of a group of three or four uninjured cells near the edge of the specimen.

Focus for the middle of one of the cells, and neglecting the hazy shadows of the parts above and below this level, sketch just what you see clearly defined. This is an "optical section" of the cell. The wall will appear as a very narrow band running round the cell defined by a fine line on the outside and inside. The area enclosed by this band at first sight appears uniformly rose-coloured, but very careful observation will show that there is an exceedingly inconspicuous layer of material-the protoplasm-between the 
rose-coloured vacuole and the wall. This layer may at some places be thicker and may be seen to be colourless. In one of these thicker places the nucleus may occasionally be seen.

When your sketch is made replace the low power and apply a drop of a Io per cent. salt solution to the edge of the cover. Be careful not to let the salt solution run over it and try not to displace the slide. In a few minutes you will observe the plasmolysis of the cells. This will be apparent even with the low power. Notice that the colour of the vacuoles becomes much more intense, and that the pigment forms globules within the cells. If your cover is still quite clean put on your high power. Find again the group of cells you sketched previously and make a new skettch, noting the changes which have come about.

Next replace the low power, remove the slide, take off the cover-glass and lift the specimen into a small glass of clean water. Mount it afresh in a drop of clean water and cover. You will now be able to observe that many of the plasmolysed cells will gradually recover their original condition.

Heat a specimen mounted in water and one mounted in ro per cent. salt solution cautiously over a Bunsen flame. When the slide becomes unpleasantly hot to the touch, examine with the microscope. Note any changes you observe in the colour of the vacuole or in the appearance of the protoplasm. 


\section{LECTURE III.}

IN the study of the cells of the leaf-skin and of the beetroot (or rhododendron petal) very important properties of the materials which compose cells have come to light. The knowledge of these properties explains how the substances perform various functions which are assigned to them in the cell. Thus the tenacity of the cell-wall supports the plastic protoplasm when the semi-permeability of the latter obstructs the passage of dissolved particles. So the rigidity of the cell is secured by a tension member, the cell-wall, and a compression member, the mutually repelling dissolved particles in the vacuole. The rigidity of tissues and of many organs is explicable by the rigidity of their component cells. Hence it is that, when the osmotic pressure of the cell vacuoles of a herbaceous tissue or organ is relieved by making the semi-permeable protoplasm permeable by heat, the rigidity of the whole tissue or organ disappears.

The dissolved substances in the vacuoles which give rise to this osmotic pressure and force the protoplasm out against the cell-wall are very various. Chemical tests show that they may be organic and inorganic acids and their salts, also carbohydrates. In addition to these bodies which diffuse and generate osmotic pressure, there are also found in the vacuole other bodies which have not got these characteristics. The former are said to be in the crystalloidal state, while the latter are described as being in the colloidal state. These names were applied because when the water, throughout which the former substances are distributed, is evaporated, the substances crystallise out, while under similar circumstances the latter form a colloidal or glue-like mass. The most important substances which are found in cell vacuoles in the colloidal state are proteins, dextrins and gums.

The difference of these two states with regard to diffusion may be easily shown by experiment as follows: In this jar there has been placed a small quantity of a ro per cent. "solution" of gelatine mixed with some Congo red-a collodial dye. In its fellow is some of the same gelatine solution to which had been added some copper sulphate. In each case care was 
taken to avoid splashing the sides of the jars with the coloured mixtures. When the gelatine had set the jars were filled up with water without fear of stirring up the coloured mixtures. After a few days it could be seen that the blue of the copper sulphate had diffused upwards into the water, its surface rising above the gelatine was no longer clear-cut, but merged gradually into the water above. This condition of things is still plainly visible. In contrast to this the colloid has not risen or left the gelatine, and its surface remains as clearly defined as it was at the beginning of the experiment. Colloids do not diffuse in water, and hence they do not produce osmotic pressure as crystalloids do.

Another simple experiment brings out a very important difference which exists between substances in the crystalloidal and colloidal state. Here are two rectangular glass vessels. In one is a solution of common salt and into the other a mixture of I per cent. gelatine in water, which will serve in this case as an example of a colloid. ${ }^{1}$ By suitable arrangements a beam of light from an arc lamp is passed in succession through the two vessels. You will see that while the path of the beam is clearly marked out in the mixture of the colloidal gelatine, it is practically invisible in the solution of the crystalloid. It is in fact only visible in the latter when it is reflected from the surface of minute specks and dust particles floating in the latter. The distinct visibility in the colloid mixture, on the other hand, is to be attributed to the reflection of light from the surface of the excessively minute colloidal particles suspended in the water. This shows that there is a difference in the manner in which the colloid and the crystalloid are distributed. The particles of the former, though still almost inconceivably small (they are quite invisible to the highest powers of the microscope), are sufficiently large to reflect light from their surfaces; whereas in the case of the dissolved crystalloid, subdivision is so much more minute in comparison with the wave length of light that there are no surfaces between the dissolved substance and the solvent capable of scattering light. Here then is a most important difference between matter in the colloidal and crystalloidal states. The colloidal particles are sufficiently large to have a surface between the solvent and the colloid. In the

1 The gelatine should first be swelled in cold water and afterwards hot water added to make up the mixture. The mixture should be thoroughly stirred and mixed. It will look then scarcely less clear than the solution of salt. 
case of the dissolved crystalloid the particles are so minute and the union between the solvent and solute is so intimate, that the phenomena which are characteristic of a surface do not occur. It is evident then, that the mixture of a colloid in water is very different from a true solution. To mark this difference a colloid is said to be dispersed, while a crystalloid is said to be dissolved in the medium which contains it.

The difference in size of the particles of matter in the two states may be further demonstrated by filtration. It is found that characteristic colloids will not pass through a vegetable or animal membrane, whereas a solution of crystalloids may be transmitted easily. This fact, which is utilised in dialysis to separate crystalloids and colloids, shows clearly the different state of subdivision characteristic of the two states. Thus certain colloids-especially the proteins-may be broken into smaller aggregates which will pass through a cellulose membrane, but yet are not small enough to exhibit all the characters of dissolved crystalloids. A complete series of preparations, grading into each other, may be made, in which the lowest members of the series are true solutions (diffuse, exercise osmotic pressure and penetrate organic membranes) while the upper members have all the characteristic properties of true colloids.

Colloidal particles although invisible with ordinary microscopic methods may be rendered visible by the ultra-microscope, an apparatus by which particles may be microscopically viewed while reflecting a very intense beam of light.

Particles having a diameter of $10^{-5} \mathrm{~cm}$. are scarcely visible with ordinary microscopic methods and at this size their surface is so large compared to their volume that they begin to exhibit phenomena unlike larger visible particles. The smallest colloidal particles must exceed molecular dimensions (viz. about $10^{-8} \mathrm{~cm}$.). Therefore the diameter of colloidal particles may be said to range between $10^{-5}$ and $10^{-8} \mathrm{~cm}$. Particles having a greater diameter form suspensions. And when subdivision is as fine or finer than the lowest limit mentioned the mixture has the properties of a solution.

It is evident that, as the proportion of surface to bulk rapidly increases with diminution in size (the volume of a sphere depends on the cube while its surface depends on the square of its radius), the surface of a given mass of matter in the colloidal state is relatively enormous and we may expect peculiarly enhanced effects in dispersed colloids of all actions which take place at surfaces. Thus when a dissolved substance tends to 
concentrate on a surface in contact with a solution, this surface condensation, or adsorption as it is called, becomes very important in the case of colloids.

In a liquid dispersion of gelatine in water, the water forms the continuous phase, i.e. it is continuous, while the gelatine, which is in the form of excessively minute, isolated particles, is the discontinuous phase. As more and more water is abstracted from such a dispersion, a critical concentration is reached when the condition of things is reversed and the gelatine becomes the continuous phase, holding the water enclosed in it as a dust of minute particles. While the water is the continuous phase the mixture acts like a liquid and is called a sol, but when the gelatine is continuous the mixture resembles a solid and it is called a gel. The transition from sol to gel at any given concentration of the colloidal substance depends on the temperature. Thus a dispersion of gelatine which is a sol at $40^{\circ} \mathrm{C}$. may be a stiff gel at $20^{\circ} \mathrm{C}$. An item of great interest attaches to the melting-point of a gel. When the temperature at which a gel breaks down and becomes a sol is noted and compared with that at which the same dispersion becomes a gel on cooling, the two do not correspond. The melting-point is different according as it is approached from a higher or a lower temperature. In fact, the past history of the colloidal mixture controls its behaviour.

In the cells of plants cellulose, starch and sometimes protoplasm are in the gel state and so may be compared to a honeycomb enclosing droplets of water. The proteins and other colloids of the vacuole and often protoplasm are examples of the sol state, and are distributed through the water as an inconceivably fine dust. The colloid in either condition presents an enormous surface. The gel state may pass automatically by simple dilution into the sol condition, and vice versa by concentration pass from the sol to the gel condition. Vegetable gums furnish examples of these changes. Sometimes such reversal is dependent on heat, as in the case of gelatine. Another change, which is exhibited by some bodies in the colloidal state, is coagulation or precipitation. It is shown in a characteristic manner by proteins and protoplasm. In it the transparent sol or gel becomes opaque and the colloidal substance aggregates like a precipitate. Again, if a semi-permeable membrane formed of a protoplasmic gel is coagulated it becomes permeable. Coagulation may be brought about by heat. A temperature of about $70^{\circ} \mathrm{C}$. is sufficient to coagulate many proteins and protoplasm. Here may be recalled the observation 
that when heated the semi-permeable protoplasm of the beet-cell became permeable and let the dissolved pigment escape. Coagulation may also be effected by chemical means, or even by the presence of other colloids. There is reason to believe that coagulation is sometimes due to the neutralisation of electric charges on the colloidal particles.

The cells we have hitherto examined belonged to great masses of cells forming the tissues of higher plants. Being composed of aggregates of cells, these plants are called multicellular plants. More simple plants are known consisting of fewer cells: and the simplest of all are those which, consisting of a single cell, are. termed unicellular plants.

To one of these we will now turn our attention.

It is the well-known plant which is utilised in the rising of bread and in the brewing of beer-the Yeast plant. Supplies may be easily obtained from a brewery or from any shop which supplies yeast for bread-making.

Yeast as thus obtained is a plastic buff-coloured mass. It is friable and for microscopic examination a fragment the size of a pea may be broken off and shaken up in a small test tube containing a sugar solution (say Io or I 5 per cent.). After continued shaking the mass of yeast disappears and is distributed through the sugar solution, forming a milky suspension.

If a drop of this suspension is placed on a slide and covered with a clean cover-glass and examined with the low power, a vast crowd of very minute specks will be seen. These specks are uniform in size and have a very definite outline. Each is, in fact, a single cell of yeast, and each cell constitutes a complete plant. This yeast plant is known botanically as Saccharomyces cerevisea.

By applying the high power something more of the plant's structure may be made out. Even with the high power it still appears very minute. When carefully measured it is found to be an egg-shaped cell, just attaining the $1 / 100 \mathrm{~mm}$. in its longest diameter. As the cells are very uniform in size this yeast plant is very useful as a standard of microscopic measurement.

When the focus is adjusted to the equator of one of these unicellular plants it is possible to see the very sharp line which limits the outside of the cell. This line represents the outer surface of the cell-wall. The thickness of the cell-wall can scarcely be made out without a special procedure. Within the cell-wall is the protoplasm of the cell. When the optical section, or focal plane, coincides with the equator of the cell one can see that there is a zone of brilliant or highly refringent material 
extending from the cell-wall inwards and occupying nearly all the space encircled by the wall. This is the protoplasm of the cell. There are often one or more circular patches in the section, of less bright substance, surrounded by the protoplasm: these are vacuoles. Very often, when examined in bright daylight, the protoplasm appears pale bluish, while in contrast to it the vacuoles seem duller and tinged with pink. By frequent readjustments of the mirror, the focus of the condenser and the aperture of the

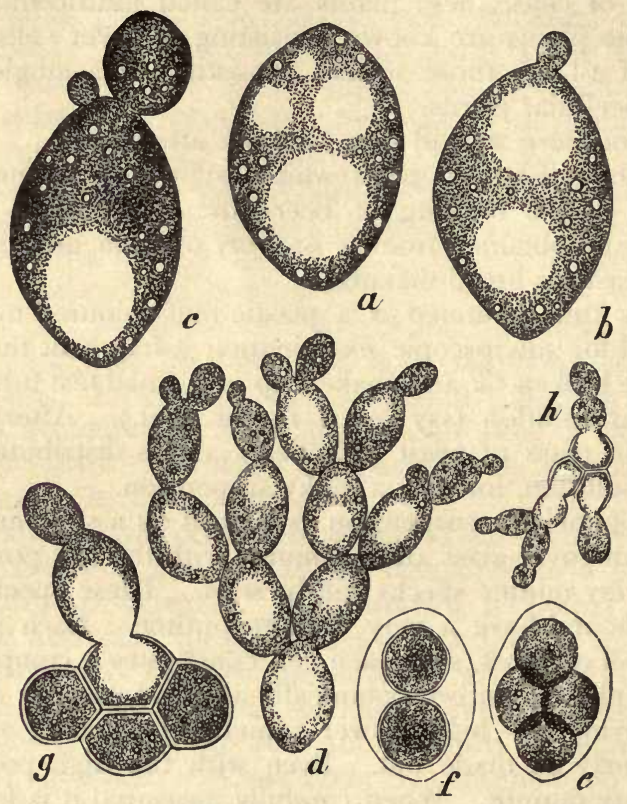

FIG. 5.-Saccharomyces cerevisea. $a, a$ single plant, $\times 2500 ; b, c, d$, budding plants; $e, f$, formation of spores; $g, h$, germination of spores. (From Evans: An Intermediate Textbook of Botany. After Reess.)

diaphragm you will arrive at clearly distinguishing these structures, and also during these manipulations you will discover, most probably, various very minute specks in the protoplasm and even in the vacuole. Where specks are seen in the vacuole they usually show the peculiar dancing motion which they, in common with all very minute bodies suspended in fluids, exhibit. This motion is considered to be due to the impacts of the molecules of the medium on the particles and becomes apparent when the 
mass of the particles is sufficiently minute to be appreciably disturbed by these impacts. It is called Brownian motion.

The cell-wall may be rendered more plain if some yeast is mounted in a ro per cent. solution of common salt. The protoplasm is then driven in from the walls by the osmotic pressure of the salt solution and the cell-wall appears standing out alone, and its clear colourless nature may be observed. Again, if the coverglass of a preparation of yeast is pressed firmly and ground to and fro on the slide, some of the cells will be ruptured and the more or less completely emptied cell-walls may be observed in the preparation. Their toughness and elasticity may be observed.

While the majority of the yeast plants are very uniform in size, some cells in the suspension will be found which are much smaller than the majority: also some of the larger cells will be found with these smaller cells in contact with them. By observing a number of these small groups it will soon appear that a smaller cell arises as a minute lump or excrescence on a mature cell. This swelling grows bigger and bigger until it attains the size of the supporting cell. While the growing cell or bud increases in size, its place of attachment remains small, and at some time in its growth the protoplasm in the bud is cut off from the original cell by the completion of the cell-wall. In this stage the bud is very easily detached and is set free by any disturbance in the medium. When the medium is undisturbed many generations of buds may adhere together. In a solution kept at $30^{\circ} \mathrm{C}$. it is observed that about six hours elapse between the beginning and the maturity of a budded cell. This budding is a most prolific method of reproduction.

Occasionally one finds in yeast preparations an unbroken cellwall containing three or four smaller complete cells. By special methods of culture this type may be produced at will. The inner cells are formed by the breaking up of the protoplasm of an original cell into a small number of masses and each of these masses becoming covered with a cell-wall. Each of these enclosed cells is called a spore. It is capable of becoming a new plant.

Yeast surrounded with a sugar solution and kept at a temperature of about $30^{\circ} \mathrm{C}$. grows rapidly. The individual cells multiply and the buds grow quickly to maturity. Its rapid growth is marked with the formation of bubbles of carbon dioxide and the production of alcohol. If the temperature is lowered the rate of growth is retarded, until at about $8^{\circ}$ or $10^{\circ} \mathrm{C}$. it ceases. Above $30^{\circ} \mathrm{C}$. growth falls off and stops at about $60^{\circ}$ or $70^{\circ}$. The 
temperature round which growth is most vigorous is called the optimum temperature. At certain upper and lower limits growth ceases and finally death supervenes. The upper limit where death supervenes is, for ordinary yeast, about $70^{\circ} \mathrm{C}$. The spores, however, may resist a higher temperature and this higher limit of the spores may be further raised by desiccation. Dried spores may actually grow after being exposed to a temperature of $120^{\circ} \mathrm{C}$. This greater resistance of the desiccated spores is probably connected with the fact that desiccated proteins resist coagulation while imbibed proteins at the same temperature are coagulated.

The enlargement of existing cells and the development of new ones require material. New wall substance-cellulose, new protoplasm, new vacuoles must be formed. The crude material to be converted into these structures must be available. This material must be supplied in the medium in which the plant grows. In breweries this medium is the sweet wort-composed of the germinated barley seeds. It may be regarded as a mixture of proteins, carbohydrates and salts. These are modified and absorbed by the yeast plants and converted into the materials needed for growth.

Such conversion is called assimilation.

\section{PRACTICAL WORK.}

Mix up a I per cent. sol of gelatine (swelling the sheet gelatine first in cold water). Observe it by transmitted light, and in a concentrated beam of light. Compare the behaviour of a salt solution. Mix up a ro per cent. sol of gelatine. Observe how the sol sets to a gel when the temperature falls. On a rise of temperature the sol state is again assumed.

Shake the gel in a test tube and notice its elasticity. Violent shaking may fracture the gel. When the fractured surfaces come together again the fracture is healed.

Shake up a little oil of cloves in water in a test tube. An emulsion results which is whitish in colour. When left to stand the droplets run together and rise as larger drops to the surface leaving the clear oil floating on the clear water. When the oil is dispersed as droplets in "the water it scatters the light penetrating the mixture and the latter looks white.

Now dissolve 2 drops of oil of cloves in ro c.c. of spirit. Add the solution to 300 c.c. of water. Notice the appearance of a very faint bluish coloration. The mixture looks clear to transmitted light, but a concentrated beam passing into it appears brilliant blue. The droplets of oil are here reduced to the dimensions of the colloidal state.

Shake up some yeast in a little sugar-solution and make a suspension. Notice the production of gas in the suspension and the smell of beer which develops. Mount a drop of the suspension on a slide. Draw carefully an ovoid cell showing the protoplasm and the vacuole. Draw cells showing the different stages in the development of a cell by budding. Mount some yeast in a ro per cent. salt solution and observe plasmolysis. Crush a preparation of yeast and look for emptied cell-walls. 
Smear some of the yeast suspension on a clean cover-glass. Leave it to dry. When perfectly dry pass the cover-glass quickly two or three times through a Bunsen flame with the smeared side uppermost. Put a drop of watery gentian violet on the smear and leave for five minutes. Wash off the stain under a jet of water, shake off as much water as possible and rinse with a few drops of spirit. Shake off the spirit and leave to dry. When perfectly dry add a drop of Canada balsam to the smear and invert it on to a slide. This smear-preparation should show scarcely any trace of colour when complete. Under the microscope the protoplasm should appear faintly tinged and a dark speck in it indicates the presence of a nuclear structure. 


\section{LECTURE IV.}

YEAST, then, lives in a mixture of proteins, carbohydrates and salts in water. Evidently the soluble salts and carbohydrates being free to penetrate the cellulose wall of the cell by diffusion come into contact with the protoplasm and are by it taken up and incorporated. Not so, however, with the proteins and insoluble carbohydrates. Their dispersed colloidal particles are, for the most part, too large to penetrate the colloidal cell-wall and consequently cannot come directly into contact with the protoplasm.

As was mentioned before, proteins are capable of partial disintegration furnishing substances of less molecular complexity and smaller molecular weight. These substances, the peptones and amino-acids, exhibit some of the properties of crystalloids and appear to be intermediate in their characteristics between crystalloids and colloids. Chemically the change is one of hydrolysis, and may be effected by means of acids. The plant, however, effects it by the agency of bodies called proteolytic enzymes.

The enzymes, of which these are a special group, are substances which are produced by organisms to hasten or facilitate a change. The change which is thus facilitated would otherwise take place imperceptibly slowly, at ordinary temperatures. In the presence of the enzyme, however, the change proceeds with moderate speed. At the end of the change the enzyme is unaltered, except so far as secondary reactions may affect it. The enzymes resemble in their action catalysts, which in a similar way determine and hasten slow reactions. But these organic catalysts differ from the inorganic, e.g. acids, platinum black, etc., in being limited in their action, and each will catalyse only one, or a group of similar reactions. For this reason they are said to be "specific". There is, in fact, reason to believe that the atomic groups in the enzyme have a specific relation to those in the substance on which it acts. In other words, the molecule of the enzyme " fits" the molecule of the substrate, as the substance to be changed is called.

Enzymes are in the colloidal state but their degree of dispersion varies, hence some of them can penetrate a cell-wall and others 
cannot. Like colloids many are coagulated by heat, and this coagulation being irreversible the enzyme is no longer active. Many may be coagulated by alcohol, and when this coagulation is reversible, the enzyme thus precipitated may be again dispersed in water and is able to carry on its action. Thus extraction with water and coagulation with alcohol form a very useful method in the production of enzyme preparations.

It is now very generally held that the reactions which enzymes accelerate are reversible actions, i.e. reactions in which a disintegrating and synthesising action are going on simultaneously, and in which the final condition is a state of equilibrium, or balance struck, between these two actions. The enzyme accelerates both actions, and consequently the final condition is the same whether attained automatically or by the aid of the enzyme. When either of these actions is much more rapid than the other the change appears complete or almost so; when the two are more evenly balanced the change is incomplete. Suppose, for instance, an enzyme is in contact with a substrate A and accelerates its hydrolysis so that it disintegrates into two products $\mathrm{B}$ and $\mathrm{C}$, and at the same time the enzyme accelerates the combination of $\mathrm{B}$ and $\mathrm{C}$ into $\mathrm{A}$. At the beginning of the reaction only a little of the two products has been formed. Consequently but little synthesis takes place and this synthesis is completely dominated by the formation of these two products under the action of the enzyme on the large quantities of A still available. But as the reaction goes on, owing to disintegration, there is less of $\mathrm{A}$ available and consequently less of $\mathrm{B}$ and $\mathrm{C}$ is formed, while the synthesising action increases as $\mathrm{B}$ and $\mathrm{C}$ become more abundant. Evidently an equilibrium point will be reached where the two opposing actions will balance each other. It is also clear that the relative rates of synthesis and disintegration will determine whether the reaction will appear incomplete or complete. Evidently, if the products of one of the actions are continually removed from the reaction as quickly as they are formed, the change will become complete in that direction. This condition seems to be often realised in the changes effected in the metabolism of plants, so that an enzyme which, under ordinary laboratory conditions where the products are not removed, acts as a disintegrating agent may in the plant cell if all the products of synthesis are removed act as a synthesiser.

Being in the colloidal state enzymes present enormous surfaces in the water in which they are dispersed, and the 
substrates and the products become concentrated on this surface. This surface concentration or adsorption seems essential in enzyme action. As colloids their actions are readily localised in the cell since they will not tend to become distributed throughout the substance of the colloidal protoplasm. It is also known that the action of some enzymes is greatly enhanced by the presence of certain dissolved substances while the existence of inhibiting substances is also indicated. These characteristics render enzymes extraordinarily suited to the delicate and localised actions needed in the cell. We may easily imagine, granted agents with these characteristics, how very varied reactions may take place in close proximity to one another but so completely isolated from one another in the colloidal protoplasm as not to interfere in the slightest. The enzyme by means of which yeast accelerates the hydrolysis of proteins has been called endotryptase. Possibly this substance is a mixture of several enzymes as its action extends to several different proteins disintegrating them into amino-acids, and thus rendering them accessible to, and capable of assimilation by, the yeast cell.

Invertase is another enzyme produced by yeast. It is produced very widely throughout the vegetable kingdom and being easily extracted lends itself to experiment apart from the cell. Invertase is responsible for the reversible change of cane sugar into dextrose (or glucose) and levulose, which may be indicated as follows :-

$$
\underset{\text { Cane sugar. }}{\mathrm{C}_{12} \mathrm{H}_{22} \mathrm{O}_{11}}+\underset{\text { Dextrose. }}{\mathrm{H}_{2} \mathrm{O}} \rightleftharpoons \underset{\text { Levulose. }}{\mathrm{C}_{6} \mathrm{H}_{12} \mathrm{O}_{6}}+\underset{\text { Cen }}{\mathrm{C}_{6} \mathrm{H}_{12} \mathrm{O}_{6}}
$$

Under normal conditions the change is complete or almost so. The final product, being nearly entirely a mixture of the two hexoses (dextrose and levulose), is called invert sugar. The change itself is called inversion. It is easily studied owing to the fact that cane sugar does not react with the blue alkaline copper tartrate solution (Fehling's solution) to produce the red copper oxide, while invert sugar does this when heated with that reagent. Thus a solution of cane sugar, which may be tested with the Fehling's solution beforehand, will after the action of invertase give this reaction.

The invertase may easily be obtained by adding a few cubic centimetres of toluene to $\mathrm{roo}$ gm. of pressed yeast and keeping the mixture in a loosely stoppered bottle for three weeks at a temperature of $30^{\circ} \mathrm{C}$. The toluene kills the yeast without injuring many of the enzymes which it contains. The proteolytic enzymes destroy the cells and the greater number of other 
enzymes, leaving over a slime containing a few active enzymes among which is invertase. A muddy sediment settles from this slime and if the supernatent clear liquid is decanted off, it is found to be an active preparation of invertase. The usual destructive effect of a high temperature may easily be verified with this preparation. When raised above $70^{\circ} \mathrm{C}$. it will be found that the enzyme has become inactive. The addition of alcohol to the preparation precipitates, or coagulates, the enzyme; but this, however, recovers its activity when redissolved in water.

Invertase is one of those enzymes, which, working under the ordinary laboratory conditions, effects an apparently complete change of sucrose (cane sugar) into dextrose (or glucose) and levulose: but it is quite possible that in the spaces of cells, where all the sucrose or water is continually removed from the sphere of activity of the enzyme, that a synthesis of sucrose from dextrose and levulose may also be effected.

Slow penetration of the diffusible peptones, amino-acids, sugars and salts makes up for those which are absorbed by the protoplasm; within the protoplasm they are further modified and built up into new protoplasm. This action, which is called assimilation, is a characteristic of protoplasm and is not exhibited by dead matter. Probably in many of the stages of . assimilation enzymes are used as agents.

Evidently, if assimilation alone is considered, new additions to the protoplasm, vacuoles and substance of the cell-wall are continually being made. These additions become visible in the growth of the cells and their reproduction. It is to be noted in the case of yeast that the carbon as well as the nitrogen is supplied from organic sources. Carbon is present in the medium round the cells in the form of proteins and carbohydrates, and nitrogen principally in the form of proteins. It has been found, however, that yeast can draw its supplies of nitrogen from simpler organic compounds such as ammonium tartrate.

For this assimilation, or building up process, in which more complex bodies are formed from simpler ones, and bodies which contain more energy from those which possess less, evidently a supply of energy is needed.

This supply of energy is furnished by combustion.

We noticed already that when yeast is immersed in a sugarsolution gas is evolved and the smell of alcohol may be observed. As a matter of fact the change indicated by these products may be expressed chemically as follows :-

$$
\mathrm{C}_{6} \mathrm{H}_{12} \mathrm{O}_{6}={ }_{2} \mathrm{C}_{2} \mathrm{H}_{5} \mathrm{OH}+{ }_{2} \mathrm{CO}_{2}
$$

Glucose or dextrose $=$ ethyl alcohol + carbon dioxide. 
The sugar disappears and the production of alcohol and bubbles of carbon dioxide may be observed. This change makes energy available, though how at first sight may not appear evident.

It is well known that when coal is burnt, heat is given off, which may be utilised in a steam engine to do work. Evidently the combustion of the coal sets free energy which is utilised by the engine. The amount of energy available may be estimated by the amount of heat produced. Using the usual standard this heat may be measured by the number of kilograms (litres) of water it can raise through $\mathrm{I}^{\circ} \mathrm{C}$. Thus one gram of coal, on burning, can raise 7 to $8 \mathrm{~kg}$. of water through one degree. In other words, it can generate 7 to 8 Calories. In the same way I gm. of glucose on complete combustion can generate about 3.76 Calories. On complete combustion the glucose is entirely converted into carbon dioxide and water. In the process, however, which we are considering combustion is not complete. One of the products is ethyl alcohol which in itself, as is well known, may be further oxidised, and may, in the oxidation, set free a considerable amount of heat. From the equation quoted above it appears that one gram-molecule of hexose $(\mathrm{r} 80 \mathrm{gm}$.) produces two grammolecules $(92 \mathrm{gm}$.) of alcohol. The fact that both bodies are in solution must also be allowed for, so that it is found that, during this incomplete combustion of hexose to alcohol, for each I $80 \mathrm{gm}$. of hexose $92 \mathrm{gm}$. of alcohol is formed, and that while the original hexose in solution had a heat of combustion equal to about 300 Calories, the heat of combustion of the resulting alcohol in solution amounts to only about 145 Calories. The remaining r 55 Calories have been set free and are available to do the work of the yeast cell.

Reactions occurring within the cell, which like the foregoing are a source of energy, are described as respiration. Generally these reactions are of the nature of oxidations, i.e. are combustions, and most usually they are complete and utilise free oxygen; but often they are incomplete and utilise combined oxygen as in the yeast cell. Respiration utilising combined oxygen in this way is called anaerobic respiration, inasmuch as the oxygen used is not drawn from the atmosphere. Very usually the substances oxidised are sugars as in this case.

Inasmuch as assimilation, and other processes which require an expenditure of energy, are characteristics of protoplasm it is natural to find that all active protoplasm exhibits respiration in one form or another. It is a general characteristic of protoplasm, 
and the energy thus rendered available becomes manifested in the various phenomena which we call life. It is only in connection with the substance protoplasm that these phenomena of energy are exhibited. Thus it has been said, "Protoplasm is the physical basis of life". Life appears to be the complex energy transformations and material changes associated with protoplasm.

The process of anaerobic respiration in yeast is then identical with the process of fermentation or the conversion of sugar into alcohol. The commercial value of yeast centres, of course, on this process. For a long time it was held that fermentation was a property of the protoplasm itself, and that the process could not be carried on apart from living protoplasm, and in fact it was one of the "vital" attributes of the cell. Consequently great interest was aroused when it was discovered that a substance could be extracted from yeast which, apart from all living protoplasm, could bring about, or accelerate, the change of sugar into alcohol and carbon dioxide. The substance is zymase, and it is found to act in every way like other enzymes. Thus we come to regard respiration as a process dependent on the still more general characteristic of protoplasm, that of producing organic catalysts-the enzymes - by means of which the protoplasm can effect changes which apart from these bodies can only be brought about by the application of powerful reagents or of high temperatures.

The number of enzymes extracted from yeast cells. is already considerable and it is likely that these cells possess many more not yet recognised: indeed many, if not most, of the chemical reactions of cells seem accelerated and controlled by these agents which often appear to be formed only when their need arises.

\section{PRACTICAL WORK.}

Dissolve a little cane sugar in about 20 c.c. of hot water, add $2 \mathrm{gm}$. of gelatine previously swelled in cold water. When the gelatine has completely gone into "solution" and the mixture has cooled to about $30^{\circ}$ add a drop of a yeast suspension. Distribute the yeast thoroughly by shaking. While still liquid put drops of this mixture on slides and cover. If the preparation is successful you will see two or three isolated yeast cells in each field of the high power. Subsequent observations will show the successive stages of growth and budding. As the gelatine sets to a gel there is no danger of the successive buds being dislodged from the positions in which they originate, and one can see the successive generations formed during the time of observation. The time occupied from inception to maturity may be noted, by keeping one cell under periodic observation.

Before leaving the yeast cell it will be well to utilise it to calibrate our 
apparatus for microscopic measurement, the Ghost-micrometer. ${ }^{1}$ With a mature cell in focus prop a transparent squared screen or even a piece of fine wire gauze against the front of the stage of the microscope with its lower edge resting on the table. The sides of the meshes of the screen should be about $2 \mathrm{~mm}$. apart. Using the flat mirror, adjust the condenser so that the yeast cell and the image of the meshes are simultaneously in focus. Alter the inclination of the screen till the meshes appear perfect squares. Now note how many yeast cells would fit along the sides of the images of the square meshes. The number may be about eight or ten. We already know from the measurements recorded by investigators that the diameter of a yeast cell is approximately $0.01 \mathrm{~mm}$. Hence we

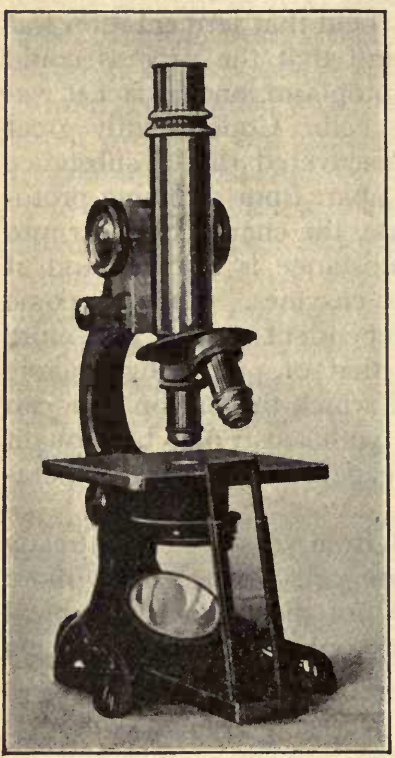

FIG. 6-Microscope with Ghostmicrometer in position. know the size of the image of the meshes in the position where we have propped it; and whenever we wish to estimate the size of a microscopic object in the field of the microscope, we have only to replace the micrometer in the same position and observe over how many meshes or portions of meshes the object extends.

The Ghost-micrometer also gives much help in drawing. The relation of objects to one another and their proportions in size to one another in the squared field produced by the Ghost-micrometer may be readily transferred with accuracy to squared paper.

More accurate work may be done by calibrating with a slide carrying a millimeter divided to roo parts.

The experiments described in the lecture on invertase should be repeated. Make up a 5 per cent. solution of cane-sugar (sucrose). Pour out a little into a test tube and add an equal volume of Fehling's solution. Boil the mixture. Is a precipitate formed? Now make a mixture of equal volumes of the sugar solution and of a 5 per cent. solution of the invertase preparation described in the lecture. Leave for ro minutes. Then test some of the mixture with an equal quantity of Fehling's solution. Immediately on boiling an orange-red precipitate appears. This is due to the reducing power of the invert sugar (levulose and dextrose) formed by the inversion of the sucrose. A separate test will show that it is not due to a reaction between the copper solution and the enzyme.

Now boil some of the enzyme preparation, cool, and add to an equal volume of sucrose solution, leave for ro minutes as before and again carry out the test with Fehling's solution. No orange-red precipitate appears. The boiling of the enzyme ras destroyed its power of inversion.

${ }^{1}$ A good form of this simple measuring apparatus may be obtained from Messrs, Thos, Mason \& Co., Opticians, 5 Dame St., Dublin. 


\section{LECTURE V.}

Chlamydomonas is a very common object, but it is confused usually with so many other organisms bearing but a superficial resemblance to it that none of these objects has a special name in our language. Stagnant water often shows a green coloration; one of the commonest causes of this greenness is the presence of Chlamydomonas in the water, but it may also be due to many other organisms.

If one takes a drop of this green water, which may be found in any stagnant pool, ditch or rain pipe, and examines it microscopically, the greenness will be seen to be due to a vast number of tiny green specks, some of which are stationary, some are buzzing about and some are almost at rest but jerk about in an uneasy fashion.

First it will be well to apply the Ghost-micrometer. It shows that these little green specks are some four or five times as long as a yeast cell. They have somewhat the same shape. They are egg-shaped. Immediately we endeavour to make out details as to their structure we become impressed with the rapidity of their motion, which makes it very hard for us to observe the cell closely. By means of the Ghost-micrometer and a stop-watch it is easy to determine that the cell travels at the rate of about $O \cdot I$ $\mathrm{mm}$. in $2 \mathrm{sec}$. or about $\mathbf{I} \mathrm{mm}$. in 20 sec., which is about the same speed as the tip of the minute hand of a large watch. The speed is by no means uniform, and much higher and lower speeds may be registered.

For observation of the structure we must select a cell at rest. In a good culture a comparatively small number will be found completely motionless, for many of those which remain quiescent for a time will occasionally betray signs of their restlessness by a spasmodic oscillation from side to side. One of these occasionally moving cells is the best for examination, inasmuch as its motions usually rotate the cell, and turn different aspects to the observer.

Like that of yeast, this cell is evidently bounded by a cell-wall, and in this case its thickness may often be seen by careful focussing . 
of the equatorial optical section. Chemical tests show that this cell-wall is of cellulose.

The space inside the wall is evidently filled with a granular substance which at first sight appears suffused with a brilliant green colour. More precise observation shows that at one pole of the cell there is a small beak-like prominence which is free from the green colour. Here also it may be seen there is no cell-wall, and the beak is plainly formed of the internal protoplasm of the cell

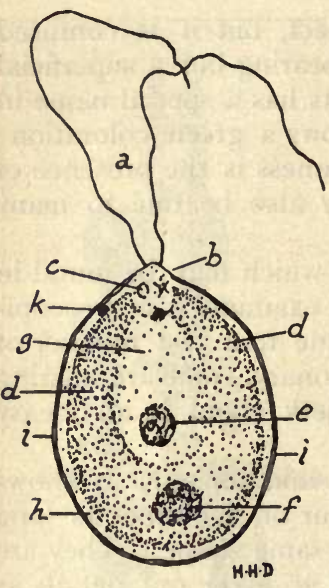

FIG. 7.-Chlamydomonas, $x$ I25 $a$, cilia; $b$, protoplasmic prominence; $c$, contractile vacuole; $d$, chloroplast ; $\ell$, nucleus ; $f$, pyrenoid; $g$, central cytoplasm; $h$, outer cytoplasm ; $i$, cell-wall; $k$, stigma or eye-spot. projecting through an orifice in the cellwall. By very careful manipulation of the fine adjustment, condenser, diaphragm and mirror you will be able to see one or two excessively fine and quite transparent threads extending out from the beak away from the cell. These threads are called cilia. They are fine extensions of the protoplasm into the surrounding water, and are about once and a half, or twice, as long as the cell. In quiescent specimens one cilium is usually comparatively easily seen. It is used to anchor the cell and is in contact with, and adherent to, the surface of the cover-glass or slide. Being at rest it is merely a matter of careful focussing and adjustment of the light to make it visible. The second cilium may be fixed, like its fellow, by its own adhesiveness to the glass. More often, however, it retains its freedom and lashes or waves to and fro. This motion makes its observation exceedingly difficult and, while free, only occasional glimpses of it can be caught, when its motion changes or when it pauses for an instant. Evidently these cilia are the organs which propel the cell through the water in the way already observed. The protoplasm composing the cilia seems quite homogeneous, it has a higher refractive index than the surrounding water and hence shows that pale, bluish coloration already observed in the protoplasm of yeast. Otherwise the cilia are colourless.

Turning our attention once more to the body of the cell we can make out the outline of a more or less spherical shape lying in 
the central protoplasm. As we focus it and examine it, it becomes evident that it is a minute bag formed of a transparent membrane within which are rather distinct granules. It is in fact the nucleus of the cell and is embedded in semi-fluid granular protoplasm occupying the central region. The colour of this central region is evidently conferred on it by the light transmitted through the peripheral regions : just as colourless water in a green wine-glass appears green. The colourless central protoplasm is continuous with the colourless protoplasm of the beak and the cilia. It will also be seen by careful focussing that a very thin colourless layer of protoplasm covers over, like a skin, the coloured zone. In fact the coloured region of the protoplasm of the cell has the form of a bell open towards the beak. This special coloured bell-shaped mass of protoplasm is called a chromatophore, and, having formed the green pigment chlorophyll, this chromatophore is styled a chloroplast. The layer of protoplasm forming the surface of the protoplasm of the cell and in immediate contact with the cellwall is free from granules, while the central protoplasm and that at the base of the beak is finely granular. At some point in the surface layer a very minute lens-shaped body may be seen. It has a very high refractive index and hence appears almost black if the diaphragm is closed, but it reveals a fine crimson colour when the diaphragm is opened and a wide cone of light illuminates the cell. This crimson body is called the stigma or eye-spot. It is usually located in the outer protoplasm near the beak or anterior end of the cell, and it appears circular or ovoid according as it is seen in plan or in profile.

While making these observations on the structure of the cell of Chlamydomonas, it is probable that the attention of the observer will be attracted by the periodic appearance in the protoplasm of the beak of one or two spaces or drops of fluid. These are at first almost invisibly small, they then grow and suddenly disappear. It is as if they slowly swelled until they passed some limit and then exploded. These are the contractile vacuoles. There are usually two, one at each side of the beak, but often they are not visible simultaneously.

The closed end of the chloroplast is turned towards the posterior pole of the cell. At this point the chloroplast is much thicker than at the open end, and occupying this thickened mass at the closed end is a structure called the pyrenoid. It is an accumulation of protein and starch. The starch is in the form of granules surrounding a central mass of protein.

If these observations have been carried out facing a good 
source of light, it will probably be noticed that the majority of the motile cells are congregating at that side of the drop of water which is nearest the light. The movement of the cells, although apparently uncertain, has resulted in bringing much the greater number to one side. It will be observed, if the slide is turned round so that the side which was furthest from the window is now nearest, that the cells will gradually face about and make their way to the illuminated side. By alternately cutting off the direct light from the window and allowing it to fall upon the preparation it may be shown that it is light which determines the direction of the motion. Here we have directed energy - the light-controlling the direction of motion of the cell. Although this cell, and many others which behave similarly, have been submitted to long and careful study, the mechanism by which this control and conversion of energy are effected has not been discovered. We cannot say that the motion of the cell is the reaction to the light acting upon it, not being able to trace the connection of the cause and effect, as we often can in inanimate mechanical systems : but we must class the phenomenon with many others among the vital (or unexplained) phenomena of cells. This is often expressed by stating that protoplasm is "irritable" and, that being so, the forces or "stimuli "acting upon it evoke "responses". In many "responses" stored energy is liberated and the action of the stimulus may be compared to the tiny expenditure of energy in the spark which explodes a mine. In such cases there is no relation between the amount of energy in the stimulus and that exhibited in the response. In the case before us it is probable that the energy received by the cell from a feeble light towards which it is moving is often very much less than that which is spent on driving the cell through the water. There is reason to believe that the stigma is an essential link in the chain connecting the light stimulus with the response.

When it is arranged that the illumination is approximately equal in all directions it will be found that the cells move hither and thither, and as we would expect, there is no massing on one side. Here the cells receive no visible directed stimulus but are free to move in any direction.

Their individual motions cannot be predicted and they appear to decide spontaneously upon their directions. This spontaneity is often assigned as an attribute to living matter. In reality there is every reason to believe that it is one and the same thing as "irritability". The cell makes all its movements in response to stimuli external or internal, great or small. An important factor 
which must not be overlooked is that the response need not be simultaneous with the stimulus which evokes it. The past history of the cell with its countless stimuli determine to a large extent its present behaviour. This so-called physiological memory is probably bound up with the colloidal nature of protoplasm and other cell-contents.

Within a culture of Chlamydomonas we not infrequently come across a large cell-wall containing four ovoid cells. These inner cells are sooner or later seen to begin to move within the large cell-wall and they finally escape as complete Chlamydomonads, in all respects like the cell we have already studied. The origin of these cells has been observed. A mature Chlamydomonad loses its cilia, its nucleus divides into two and each of these two into two more, so that there are now four nuclei in the cell. The chloroplast also subdivides and finally the whole protoplasmic mass, by two constrictions at right angles to each other, divides into four small masses, each containing a nucleus and chloroplast. A cell-wall is secreted round each mass and finally the protoplasmic beak and its cilia are formed. , When all is ready the wall of the original cell is partially liquefied (possibly by an enzyme secreted by the young cells) and the new individuals emerge.

Sometimes the protoplasmic contents of a cell break up into a much larger number of small cells so that thirty-two or even sixty-four cells may be produced. These sixty-four minute cells seem essentially similar to the larger ones. When they emerge they swim about for a longer or shorter period and then fuse in pairs. The fusion begins at the anterior ends. Each of these fusing cells is called a gamete. The mass formed of the two fused cells is called a zygote, and forms a cell-wall round itself. This cell-wall becomes thickened and a red pigment is developed in the protoplasm within. After a longer or shorter period of rest, this cell, the zygote, germinates by the protoplasm subdividing into four or eight individuals which develop walls and cilia and are complete reproductions of the cells which gave rise to the gametes. The wall of the zygote partially liquefies and the cells escape.

The former of these two methods of reproduction is described as asexual, the latter, involving the fusion of two cells, sexual. The reproductive cells formed in the asexual process are called spores. As a general rule the fusing gametes come from different cells, and in some species of Chlamydomonas it is noticed there is a difference in size between the sexual cells which fuse together. 
In very simple forms like Chlamydomonas there appears to be no hard and fast line between the spores and the gametes. Thus there are often as many as eight spores formed in the mother-cell, and as few as eight or sixteen gametes. It has been found that while spores are formed when the supply of nutriment is plentiful, starvation conditions tend to produce gametes. Again, it has been found possible to obtain new individuals from single gametes by furnishing them with rich supplies of food.

These observations have led to the speculation that sexual reproduction originated in primitive organisms as the result of starvation, and that the fusion or fertilisation which is characteristic of sexual reproduction is comparable to the ingestion of one cell by another in response to the stimulus of hunger.

\section{PRACTICAL WORK.}

Put a drop of water containing Chlamydomonas on a clean slide, cover with a clean cover-glass, and examine with the low power. Notice the unsteady motion of some of the cells; in most cases it appears to tend towards the observer. In reality the motion is away from the observer and towards the light. The motion appears reversed owing to the reversal of the microscopic image. These travelling cells rotate on their axis as they advance. Other cells are seen which are stationary: and again others which show an erratic vibratory motion. Put the Ghost-micrometer in position and estimate the size of the cells in hundredths of a millimetre.

Now take a very small drop of the water containing Chlamydomonas and add it to a drop of I per cent. gelatine on a slide and cover. The colloid gelatine restrains the activity of the cells and makes them move more slowly so that it is easier to observe their motions.

Select one of the stationary but vibrating cells. Arrange it centrally in the field with the low power. Put on the high power and examine. Note the cell-wall, the coloured region, the colourless beak, the nucleus, the pyrenoid, the stigma, the cilia, and the contractile vacuoles. By careful focussing observe the rounded near surface of the cell. By rapid minute changes of the fine adjustment, try and make out the form of the seemingly coloured protoplasm. By the manipulation of the condenser, mirror and fine adjustment, find and examine the cilia. They are only visible by their difference in refringency from the surrounding water. When seen at their clearest, they appear like very fine filaments of glass. Focus for the median optical section and carefully draw the cell, putting in all the details observed.

Put on the low power and apply a drop of liquor iodi to the side of the cover-glass, taking care not to allow it to flood the upper surface. When the reagent has penetrated well under the cover-glass note the darkened appearance of the cells it reaches. Centre one of these and switch on the high power. Observe the olive-green colour of the chloroplast. The protoplasm has become light brown. The cilia have become much more clear. The granules of the nucleus are considerably darker than the surrounding protoplasm. The pyrenoid has become dark blue. The blue coloration, in many cases, appears as a shell enclosing a dark brown material. The blue coloration is the characteristic reaction of starch with iodine, and the central brown mass is protein. 
YEAST lives surrounded with food materials so that the source of the substances which it incorporates as its food is not far to seek. The supply of food for an organism like Chlamydomonas is not so obvious. The rain-water in which it lives contains no proteins and no carbohydrates. This water is practically pure, though it may contain traces of certain mineral salts derived from the slight solubility of the mineral particles of the soil forming the sides of the pool or of the mineral dust falling into it. In this way the water surrounding Chlamydomonas may contain traces of sulphates, bicarbonates, chlorides, nitrates, phosphates, existing as salts of magnesium, calcium, sodium, potassium, and iron. Carbon dioxide is also dissolved in this water.

If a sample of Chlamydomonas be observed when exposed to strong light, it will be noticed that a froth of minute bubbles is formed at the surface. The gas contained in these bubbles is oxygen. Furthermore if the cells are microscopically examined before and after this frothing has occurred it will be found that the amount of starch in the pyrenoid has noticeably increased. To form this starch $\left(\mathrm{C}_{6} \mathrm{H}_{10} \mathrm{O}_{5}\right)_{n}$, which is a carbohydrate, evidently a supply of carbon must be available. Experiment shows that the frothing and the formation of starch only take place when the Chlamydomonas is exposed to light and when a supply of carbon dioxide dissolved in the water is available.

The conditions for the formation of starch may be more easily studied in one of the higher water plants. I have here put a handful of a very common fresh-water weed Elodea canadensis into this large glass vessel containing water in which carbon dioxide is dissolved. Bubbles will be seen to rise from the surface of the plant as soon as it is exposed to a bright light. This inverted funnel is now arranged over the weed to catch the bubbles and they, guided in their upward course, are caught in this inverted test tube previously filled with water. The bubbles. displace the water in the tube as they accumulate. Thus collected the gas may be tested. It will be found to be nearly pure oxygen. 
If the same experiment had been set up with water which had been previously boiled to remove the carbon dioxide, no bubbles would have been generated at the surface of the plant even when exposed to light.

The production of oxygen and the conditions governing it may also be studied with this plant. When a small piece is submerged in a glass vessel of water and exposed to light, a stream of tiny bubbles is seen to rise immediately from the cut end of the stem. The stream becomes feebler as we reduce the light, till it stops altogether when the light is very faint. Again the flow of bubbles becomes faster and faster as we increase the light from a faint illumination to bright sunlight. As in the previous experiment no bubbles are formed if the piece of plant is immersed in water deprived of carbon dioxide by boiling. On the screen you see the bubbles rising from the cut end of the stem. The light of the arc lamp which serves to project the image of the plant at the same time furnishes the light requisite for the production of the gas.

If we expose a culture of yeast, or an organ not having a green colour such as a root or stem, to light under the same conditions as those described above, no evolution of oxygen takes place.

It is now of interest to examine the tissue of Elodea and see how the green substance is distributed in it.

The leaves of Elodea are comparatively thin and transparent, being composed of a single layer of cells at the margin, and a small number of layers in the central regions. Down the middle of the leaf runs the mid-rib which is composed for the most part of elongated cells with long passages between them containing gas. When the leaf is submerged and viewed by transmitted light these passages look dark owing to the reflection of the light from their surfaces. These passages give off branches between the other cells of the leaf. The cells bordering the passages are roughly rectangular in form, as you see shown in this diagram. They possess a transparent cell-wall so that the contents within may be plainly seen. The cell-wall is lined by a film of colourless protoplasm of variable thickness which in turn surrounds a large colourless vacuole. Embedded in the layer of protoplasm are numbers of tiny ovoid green masses. These are the chloroplasts which confer the green colour on the leaf, and like the chloroplast of Chlamydomonas, are responsible for the formation of starch and for the production of oxygen. Embedded somewhere in the protoplasm a larger colourless body, the nucleus, may also usually be seen. It appears to possess a very definite limiting membrane within which may be seen a bright speck, the nucleolus. Con- 
centrating our attention on the chloroplasts we can see that they have a higher refractive index than the surrounding protoplasm and that the green colour is rigorously limited to their substance. Within each chloroplast one or more very minute specks can usually be seen. If we replace the water surrounding our preparation with alcohol or methylated spirit, we will soon observe that the green pigment, which was evidently insoluble in water, quickly dissolves out, leaving the chloroplasts much paler or colourless. If now a little liquor iodi be run into the preparation, the characteristic reaction for starch will be shown by the specks within the chloroplasts. Evidently the chloroplasts have been behaving just like the, chloroplasts of Chlamydomonas

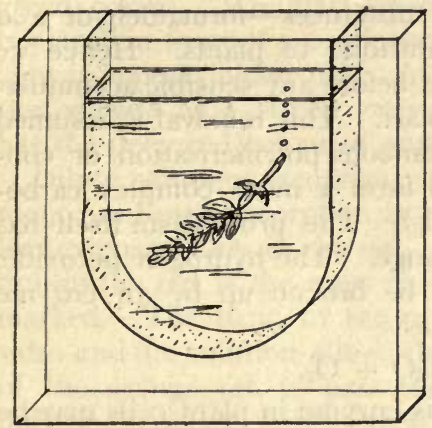

FIG. 8.-Glass cell, within which is seen submerged in water a branch of Elodea canadensis. On exposure to light bubbles of oxygen are given off.

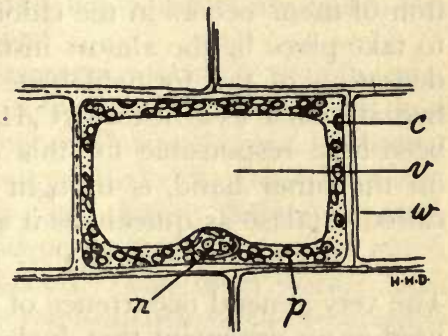

FIG. 9.-Elodea canadensis, cell of leaf, $\times 350$. $c$, cytoplasm; $n$, nucleus ; $p$, chloroplast ; $v$, vacuole ; $w$, cell-wall.

and have built up starch from the carbon dioxide and surrounding water. The oxygen set free by the process has been liberated as bubbles, either at the external surface of the cell, or injected into one of the passages of the leaf and from thence finds its way into the stem and emerges at its cut or broken surface.

The process taking place in an illuminated green cell may be represented in a simplified form as follows :-

$$
\begin{aligned}
& \begin{array}{l}
\text { Carbon } \\
\text { dioxide }
\end{array} \\
& \mathrm{CO}_{2}
\end{aligned}+\underset{\text { Water }}{5 \mathrm{H}_{2} \mathrm{O}}=\underset{\text { Starch }}{\mathrm{C}_{6} \mathrm{H}_{10} \mathrm{O}_{5}}+\underset{\text { Oxygen }}{6 \mathrm{O}_{2}}
$$

In this process the volume of carbon dioxide dealt with is equal to the volume of oxygen set free. Inasmuch as oxygen is much 
less soluble than carbon dioxide, it appears in the form of bubbles at the surface of the cell.

The equation above represents the initial and final stages of the process, but it is highly improbable that there are no intermediate steps in the formation of such a complicated carbohydrate as starch. Various steps have been suggested with more or less of plausibility attached to them, and more or less supported by collateral evidence. Thus it has been supposed that formaldehyde $\left(\mathrm{CH}_{2} \mathrm{O}\right)$ is one of those steps and that in its formation from the carbonic acid formed by the union of water and carbon dioxide, hydrogen peroxide $\left(\mathrm{H}_{2} \mathrm{O}_{2}\right)$ is produced according to the equation-

$$
\mathrm{H}_{2} \mathrm{CO}_{3}+{ }_{2} \mathrm{H}_{2} \mathrm{O}=\mathrm{CH}_{2} \mathrm{O}+2 \mathrm{H}_{2} \mathrm{O}_{2} \text {. }
$$

Experience shows that these two substances-formaldehyde and hydrogen peroxide-are highly injurious to plants. Hence we must assume that they are removed before any sensible accumulation of them occurs in the chloroplast. This removal is assumed to take place by the almost instantaneous polymerisation or condensation of the formaldehyde to form a more complex carbohydrate such as a hexose $\left(\mathrm{C}_{6} \mathrm{H}_{12} \mathrm{O}_{6}\right)$. The protoplasm itself has been held responsible for this change. The hydrogen peroxide, on the other hand, is thought to be broken up by an enzyme called catalase as quickly as it arises-

$$
2 \mathrm{H}_{2} \mathrm{O}_{2}=2 \mathrm{H}_{2} \mathrm{O}+\mathrm{O}_{2} \text {. }
$$

The very general occurrence of this enzyme in plant cells may be used as an argument that hydrogen peroxide is often produced. It may be seen that we could not expect to be able to demonstrate the presence of either the aldehyde or the peroxide by chemical tests, but it has been found that cells with chloroplasts are able to build up starch from aldehyde supplied in minimal doses. ${ }^{1}$

${ }^{1}$ It has been thought since the cane sugar (sucrose $\mathrm{C}_{12} \mathrm{H}_{22} \mathrm{O}_{11}$ ) content of some green cells rises immediately on exposure to light, while the hexoses $\left(\mathrm{C}_{6} \mathrm{H}_{12} \mathrm{O}_{6}\right)$ do not increase, that sucrose is a preliminary step to the formation of hexoses, and that by the agency of invertase the sucrose is transformed to these sugars. But it is not necessary to accept the improbable suggestion that the more complex sugar is the first formed. Supposing the hexose to arise in a very small cavity of the protoplasmic gel, or to be adsorbed upon the surface of its colloidal particles, even though in very minute quantities, its concentration might be great, and hence in presence of the enzyme a small amount of sucrose will $b=$ formed. If a mechanism exists in the cell for transporting this sucrose from the point of manufacture to a cavity or vacuole in the protoplasm isolated from the sphere of activity of the invertase, it is evident that a continual rise of the total amount of sucrose in the cell will be observed, while the amount of hexoses in it may remain very small and approximately invariable. 
Enough has been said to show you that the stages by which carbohydrates are built up from carbon dioxide and water by plants are uncertain. The process is usually called carbon assimilation, or, if it is desired to emphasise the fact that the synthesis is dependent upon light, it is spoken of as photosynthesis.

An essential and probably the most important agent in photosynthesis is the chloroplast. As we have seen, this chloroplast is a mass of protoplasm with which is associated a green pigment. This pigment is called chlorophyll. It is insoluble in water but it is readily soluble in many organic solvents, e.g. alcohol, acetone, chloroform, etc. A solution of it has very striking properties. It has a magnificent grass green colour with a red fluorescence. When viewed in thin layers by transmitted light it has a brilliant green colour. If a glass vessel containing some of the solution is placed in front of a black background and viewed with reflected light it appears blood-red. A thick layer of a concentrated solution viewed by a strong transmitted beam shows no green, but has the deep red colour of port wine.

Dilute solutions examined spectroscopically show that the pigment has broad absorption bands in the blue and violet, and four less extensive ones in the red, orange, yellow and green. Absorption in the red is the most complete while in the green it is least marked. The band in the red is very sharply defined on both sides and the solution allows the deepest red rays as well as much of the orange red to pass untouched. The other bands have indistinct margins which extend as the thickness of the solution is increased. This peculiar absorption will supply you with the explanation of the striking fact that a thin layer of chlorophyll solution in transmitted light looks green, while a thick layer of the same solution under similar conditions looks red. In thin layers the absorption stops most of the orange, yellow, blue and violet, while most of the green and the deep red are transmitted. The latter is so feebly luminous that the solution appears pure green. When, however, a thick layer is used the feeble absorption in the green becomes effective and practically all the visible spectrum is obliterated save the deep red. This latter now becomes visible when the more luminous part of the spectrum is cut off.

The fluorescence is caused by the absorption in the blue and violet. The light absorbed by these bands reappears as red light which escapes from the solution. With this fluorescence, however; we need not concern ourselves, since it is only shown by 
crystalloidal chlorophyll. Chlorophyll is in the colloidal state in the chloroplast and does not exhibit fluorescence.

The green colouring matter, as it is found in the chloroplasts, is a mixture of four pigments, two of which are green and are called chlorophyll $a$ and $b$ respectively. Chemically they are both esters of methyl $\left(\mathrm{CH}_{3} \mathrm{OH}\right)$ and phytyl $\left(\mathrm{C}_{20} \mathrm{H}_{39} \mathrm{OH}\right)$ alcohols. It has been shown that they both contain magnesium. This element is supposed to play a similar part in the chlorophyll molecule to that of iron in the molecule of hæmoglobin, the pigment of the blood. Chlorophyll $b$ contains more oxygen than chlorophyll $a$. They are represented chemically as follows :-

\section{Chlorophyll $a, \mathrm{C}_{55} \mathrm{H}_{72} \mathrm{O}_{5} \mathrm{~N}_{4} \mathrm{Mg}$; Chlorophyll $b, \mathrm{C}_{55} \mathrm{H}_{70} \mathrm{O}_{6} \mathrm{~N}_{4} \mathrm{Mg}$.}

Besides the two chlorophylls there are two yellow pigments present in the chloroplasts called respectively carotin and xanthophyll. They appear to bear a relation to one another similar to that borne by the chlorophylls to one another. Xanthophyll $\left(\mathrm{C}_{40} \mathrm{H}_{56} \mathrm{O}_{2}\right)$ contains oxygen, while carotin, the formula of which is $\mathrm{C}_{40} \mathrm{H}_{56}$, has none. These four pigments have been found in the chloroplasts of all the higher plants so far examined. They are present in about the proportions of 14 parts of chlorophyll $a, 5$ parts of chlorophyll $b$, I part of carotin and 2 parts of xanthophyll.

The exact relation of the green colouring matter to the chloroplast is doubtful. It sometimes appears as a film spread over the surface of the chloroplast while some observers believe that the green colloidal substance interpenetrates the protoplasm of the chloroplast occupying cavities within it.

As we have seen, the substances formed during photosynthesis, viz. carbohydrates, possess a large amount of energy. They may be oxidised and show a large heat of combustion. The substances from which they are built up, viz. carbon dioxide and water, are fully oxidised and can supply no energy. Hence it is evident that to effect such synthesis of substances containing a large amount of energy from ones which, from this point of view, are without any, a supply of energy is needed. This supply is evidently drawn from the energy of sunlight, absorbed by the pigments of the chloroplast, which we know is necessary for photosynthesis. In fact, we may regard the chloroplast with its pigments as a molecular engine for converting raw simple substances with a small energy content into more complex ones containing a large amount of energy. The energy for driving this engine is supplied by the sun. How these engines effect this transference of energy into the products of photosynthesis is a 
mystery: Is the combination of the raw materials effected and the energy introduced externally to, and without the alteration of the pigment of the chloroplast, just as we might suppose that a piece of clockwork might not only be manufactured from the raw materials but also wound up by an engine? or does the absorbed solar energy first make the raw materials combine with the pigments and afterwards separate off the finished product with a portion of solar energy embodied in it? Our present state of knowledge cannot answer these questions definitely, but there is experimental evidence to show that the latter is the more probable hypothesis, and the chemical relationship of the four pigments of the chloroplast makes it appear that temporary combination with them of the carbon dioxide to be used in the synthesis is probable.

To conclude, the outstanding and fundamental fact must not be lost sight of, viz. that the minute chloroplasts of plants are nature's engines for building organic substance from inorganic, and by means of them green plants not only supply themselves with food material and energy, but are actually the purveyors of food and of the energy of the sun to the whole of living nature. For naturally the food and energy which they supply to herbivorous animals is transmitted by these to the carnivora which devour them.

\section{PRACTICAL WORK.}

Cut off several short pieces from the tops of shoots of Elodea canadensis. Immerse these in rain-water containing carbon dioxide dissolved in it. Note that bubbles are discharged from the cut ends of the branches when the leaves are exposed to bright light. The stream of bubbles slows down and ultimately stops when the light is cut off. When one of these shoots is put into rain-water which has previously been boiled to remove the dissolved carbon dioxide no bubbles are formed on exposure to light.

Mount a detached leaf in water and cover. With the low power observe the general features of the leaf and note the dark lines in the central parts of the leaf. These are the air-passages between the cells. Estimate the dimensions of the cells with the Ghost-micrometer.

With the high power make a careful examination of one of the leaf cells which shows its structure plainly. Observe the colourless cell-wall. In an optical section of the vertical walls you will see their thickness. Compare the thickness of the wall with the width of the cell. Note the numerous chloroplasts lying close to the wall. Can you make out any lying near to horizontal walls? With a narrow diaphragm you will be able to make out the protoplasm like a film against the inner surface of the cell-wall. The chloroplasts are embedded in this film. Very often a nucleus can be seen. It appears as a colourless transparent body lying in the cytoplasm, as the rest of the protoplasm is called, and it has a higher refractive index than the cytoplasm. Look for its nucleolus and membrane. Notice the size of the vacuole. Study the chloroplasts and look for starch grains in their substance. Make 
measurements of the length and width of the cell and the size of the chloroplasts and nucleus by means of the Ghost-micrometer in a thin slice of the leaf.

Make a careful drawing of the cell, putting in all the structures you have observed. By careful focussing try and picture to yourself what would be the appearance of a cross section of this cell. Make a drawing of your reconstruction.

Sooner or later while making these observations, the attention of the observer will be arrested by the surprising fact that the chloroplasts of some, or of all the cells, are moving.

It will be apparent that their motion is due to the motion of the cytoplasm of the cells, which carries in its streaming motion all the substances embedded in it around the cell. Minute observation shows that the immediate surface of the protoplasm next the cell-wall does not move, while the streaming affects the deeper layers. At first when a leaf is pulled off a plant and mounted, the cytoplasm is generally quiescent and stationary in the cells, but usually after a few minutes of freedom from interference, motion gradually reappears. The first arrest of the motion is attributed to shock. As a rule motion may first be detected in some of the long cells of the mid-rib. At first it is very slow and appears almost tentative: a single chloroplast or group is slightly moved, then the motion extends to a larger piece of the cytoplasm and soon a stream is started which carries the chloroplasts and nucleus slowly up one side of the cell and down the opposite side. The streaming quickens its pace and soon attains a maximum. While these events have been taking place in one cell, similar phenomena may be seen starting in the neighbouring cells, and soon in all or nearly all of the cells of the leaf this streaming motion is in evidence so that the field of the microscope presents a striking and busy appearance. When the streaming is sluggish it may often be accelerated by gently warming the preparation. A temperature which kills the protoplasm stops the streaming. It is a phenomenon of life and is a good example of one of the so-called spontaneous actions of protoplasm. So far no satisfactory mechanical explanation of it has been given. But it is certain that to maintain it an expenditure of energy is needed. Work must be done to overcome the viscosity of the protoplasm. Thus this motion is of special interest as being one of the visible processes of the cell which consumes some of the energy acquired by the plant.

Streaming may be observed in the cells of many plants but it is seldom so marked as in the example before us. Usually the displacement of the various cell contents is very slow and imperceptible under the ordinary conditions of observation. 


\section{LECTURE VII.}

WE have seen that Chlamydomonas obtains its carbon from the carbon dioxide of the air dissolved in water. Complex carbon compounds such as proteins and carbohydrates are unnecessary for it. It builds up these compounds itself from inorganic substances. Similarly it does not require organic nitrogen-compounds to enable it to form protoplasm, but strangely enough, like other plants possessed of chlorophyll, it cannot directly utilise organic nitrogen. It draws its supplies from the inorganic nitrates and possibly, to a less extent, from the salts of ammonia dissolved in the water where it lives. In these respects green plants occupy a unique position: they alone can build up organic substances from inorganic. All other living beings require a supply of organic substances, and hence are dependent, directly or indirectly, on green plants for the food which they require to build up their bodies, as completely as they are dependent on green plants for the energy which they require for their work.

The nutrition of organisms which, like Chlamydomonas, draw their supplies of carbon and nitrogen from inorganic sources is termed holophytic in contrast to the others which depend on organic substances for their food, whose nutrition has been called holozoic.

It may be noted here that, so far as is known, no organisms can utilise elemental carbon or free hydrogen, and only few can make use of free nitrogen. Free oxygen, on the other hand, as we will presently see, enters very usually into metabolism.

When light is cut off from Chlamydomonas, as you saw, photosynthesis ceases. Observation also shows that under this condition the starch in the pyrenoid slowly disappears. Comparison with other similar plants also shows us that during this process oxygen is absorbed and carbon dioxide is formed. Evidently a carbohydrate is being oxidised and its products of combustion, carbon dioxide and water, are being eliminated. The latter is lost in the already large quantity of water in the cell and is not noticeable. You will observe that this process, which is called respiration, is the reversal of photosynthesis, and so, when photosynthesis 
is active, the products of respiration are not observable because all the carbon dioxide and water produced by respiration are built up again into carbohydrates and never appear. So that although this process of respiration is going on in the cell of Chlamydomonas without ceasing, it only becomes apparent when the reduction of light reduces photosynthesis sufficiently to allow it to preponderate.

As we have already seen the combustion of carbohydrates sets free energy. Thus I gramme molecule (or 162 g.) of starch in complete combustion changing to carbon dioxide and water sets free about 685 Calories, or I gramme of starch can generate more than 4 Calories. This energy has been previously obtained from the light absorbed by the chloroplast in photosynthesis. It is in fact solar energy. Thus if a Chlamydomonas carries a starch grain weighing $\mathrm{I} \times 10^{-10} \mathrm{~g}$. (i.e. about $\frac{1}{4}$ the size of a yeast cell) it could do work equivalent to about 0.2 gram-centimetre. This energy is used in doing the work of life in the cell, much of which eludes our observation. Some of it is visibly spent in propelling Chlamydomonas through the water, or in causing and maintaining the streaming motion of the protoplasm as in the cell of Elodea, or again in raising the temperature of the cell. During illumination some of the substance actually being formed is used up, and some of the energy being absorbed at the moment is set free for this work. In this case it is probable that a soluble sugar such as glucose is oxidised, and gives up its energy before it has been built up into starch. Some provision, however, must be made for times of darkness, during which there is still a demand for energy for various processes. This provision is secured by storing carbohydrates. There is an obvious difficulty in storing large quantities of soluble carbohydrates like sugars, since as they accumulate they necessitate a rise of osmotic pressure. In a cell like Chlamydomonas this would ultimately lead to the expulsion of part of the semi-permeable protoplasm through the anterior opening in the cell-wall. It is probably to get over this difficulty that for storage purposes the soluble carbohydrates are transformed into the colloidal state, in which their osmotic pressure practically disappears. As energy is required, the colloid starch is gradually retransformed by the agency of the enzymes diastase and maltase into glucose and is respired probably in that form. It is this conversion of starch into glucose which may be observed when Chlamydomonas is cut off from light.

But whether the energy is used directly after it is absorbed, or whether it is stored in the form of a soluble or colloidal carbo- 
hydrate, it is under all circumstances solar energy which is absorbed in photosynthesis and made available in respiration. Therefore all the work done by plants is referable to this source alone. We can generalise still further and state that all the work done by other organisms also-even by the higher animals - is ultimately to be credited to this source; for we have already seen that all organic nature is dependent on the chlorophyll of green plants for its supplies of food and that it is from the substance of these plants all other living beings draw their supplies of energy, which reappears in their work.

As in photosynthesis so in respiration, we only know the beginning and the end of the process. The stages of the reaction are unknown. Thus it is quite possible that the absorbed oxygen does not directly oxidise the soluble carbohydrates of the cell, but that it combines with the protoplasm and oxidises, in the first instance, some atomic group in it, which is replaced subsequently by the available carbohydrates. The end result is the same. For the carbohydrate is drawn on to rebuild the protoplasm and disappears just as if it were directly oxidised.

This view is rather supported by the fact that during life protoplasm is continually disintegrating, breaking down into simpler substances. These wasting processes, of which respiration is one, are classed together as catabolism. The products of catabolism cannot always be distinguished from stages in the building up processes of the cell, namely, its anabolism, and probably many of the substances found in the cell are sometimes products of catabolism and sometimes stages in anabolism. This is certainly the case with carbon dioxide and water. Evidently such an arrangement is very economical in material, but it seems only possible in those organisms which do not liberate large quantities of energy at a time. Thus it is only in the more active animal kingdom that we find special organs developed for the elimination of the waste products of catabolism.

Some view the contractile vacuoles of such plants as Chlamydomonas in this light and hold they are organs for the elimination of waste nitrogenous products from the cell. They may also evacuate dissolved carbon dioxide, which however, without their intervention, diffuses out of the cell. Certainly they act in this way; and in conformity with this view, contractile vacuoles are only found in the vegetable kingdom in motile cells in which destructive catabolic processes setting free energy are presumably very active.

It seems indeed more philosophical to regard the contractile 
vacuole not as an organ of the cell, but to view its expansions and contractions as phenomena necessitated by the continuous accumulation of soluble products at one spot in a semi-permeable gel like protoplasm. The osmotic pressure generated by the solutes presses back the protoplasm and expands the cavity in which they are, till the film separating the vacuole from the surrounding water ruptures. The solution is then free to escape. This relieves the internal pressure and the elasticity of the gel asserts itself and the vacuole collapses, its walls being forced back by the gel recovering from its distortion. As soon as the exit is closed, accumulation, proceeding as before, causes a repetition of the process. It is evident that the phenomenon will only occur in those cells whose protoplasm is not supported by a cell-wall. In others the tenacity of the wall prevents the unlimited expansion and rupture of the protoplasm. It will be noticed that among vegetable cells, it is only the motile ones which do not possess a continuous cell-wall, and hence only in motile cells would we expect to find contractile vacuoles.

Our next subjects for study are some of the organisms concerned with decay and putrefaction which we take as typifying the vast assemblage of micro-organisms known as Bacteria.

A very easy method of obtaining various examples of the different forms of cells found in this group, is to gather a little of any of the finer red sea-weeds from half-tide rocks, and to keep them covered with salt water at room temperature for two or three days. The water round the sea-weeds, which is at first clear, becomes during the first day or so more and more cloudy and turbid. When a drop of this cloudy fluid is examined microscopically it will be found to contain considerable numbers of protozoa, which may be seen swimming about in a cloud of almost impalpable specks. The specks are bacteria. When the high power is used these specks are just visible. Some of them are motionless while some are constantly moving. Of these latter some move with a steady motion, travelling for long distances in a straight line, or on a large curve; others have an uncertain gait and move hither and thither with a vacillating and flickering motion; and again others dart on a zig-zag path in a series of short dashes, pausing now and then to indulge in a giddy whirl. The specks have no distinctive colour and are apparent in the fluid owing to their high refractive index. When the diaphragm of the microscope is closed they appear like dark dust particles, when it is opened 
they are bright like specks of glass. It is easy to see that they are not all the same shape. Vast numbers of them appear as dots, and as they retain this outline no matter what their movements are, we must conclude that they are excessively minute spheres. These are called Cocci. Numbers appear as short rods with a length of 3-6 times their diameter. Usually these rods are straight (Bacilli) but they occasionally are curved (Comma bacilli). Then again we see occasionally elongated forms worming their way among the rest with a sinuous motion. These spirally twisted or corkscrew-shaped organisms are called by bacteriologists Vibriones when they consist of one or two turns; when they are longer, Spirilla or Spirochætes. When straight bacilli cohere end to end they give rise to a growth-form called Leptothrix. All these forms

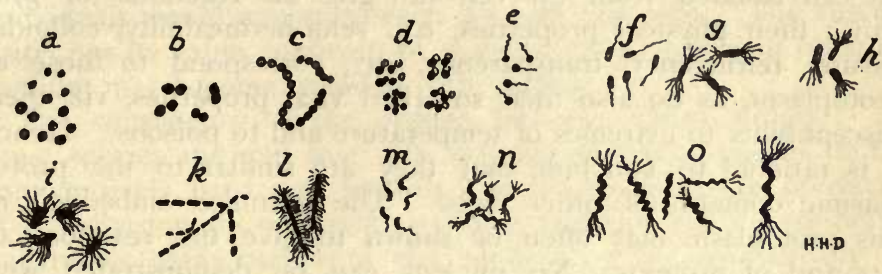

FIg. Io.-Various forms of bacteria. $a$, staphylococcus; $b$, diplococcus; $c$, streptococcus; $d$, sarcina; $e$, planococcus ; $f$, bacillus single cilium (monotrichous); $g$, bacillus with two tufts, $h$, with one tuft of cilia$g$ and $h$ are called lophotrichous forms; $i$ and $l$ peritrichous bacilli; $k$, filamentous bacilli ; $m$, comma bacilli ; $n$, vibriones; $o$, spirochætes.

are exceedingly minute, as may easily be realised if yeast cells are introduced into the fluid containing the bacteria. Some of the longest cells may attain a length equal to the yeast cell but their diameter is never more than one-third that of the yeast. In measurements of bacteria it is customary to use the micron as the unit, I micron $(\mu)=0.00 \mathrm{I} \mathrm{mm}$. Thus the longest bacillus would be $10 \mu$ long, with a diameter of I to $3 \mu$. The cocci are usually about $\mathbf{I} \mu$ in diameter. A few are even credited with a diameter of only $0^{\circ}$ I $5 \mu$. Sometimes the elongated kinds cohere together and form comparatively long rods or threads. While we may thus gain some idea of their size relative to other small organisms it is by no means easy to visualise their absolute minuteness. Calculation shows that I 7,000 million bacteria could fit in a cubic millimetre, while a single drop of liquid culture might contain 10,000 millions. In 
emphasising the extreme minuteness of these organisms you must not lose sight of their grossness when compared with molecular dimensions. Thus it has been calculated that the smallest coccus $0.15 \mu$ in diameter contains at least 30,000 protein molecules, which are of course vastly larger than molecules of simpler substances. They are in fact about the same size as the colloidal particles spoken of in Lecture II.

It is not surprising that in cells so minute as these bacteria little structure is visible. In some the protoplasm is seen to contain vacuoles and even granules may be made out embedded in it. When the cell is plasmolysed the cell-wall may be distinctly seen and it has been possible to show that the substance forming this wall is different from that of other plant cells, in that it is a protein and not a carbohydrate. The contents of the cell isolated from the cell-wall give the reactions for proteins; their physical properties, e.g. semi-permeability, colloidal nature, refringency, transparency, etc., correspond to those of protoplasm, as do also their so-called vital properties, viz. their susceptibility to extremes of temperature and to poisons. Hence it is rational to conclude that they are similar to the protoplasmic contents of other cells. The granules embedded in this protoplasm may often be shown to give the reactions of fats and of proteins. No nucleus can be demonstrated with certainty, but it is impossible to deny that some of the protein granules are nuclei, especially when they give some of the special reactions of nuclear protoplasm. Naturally the structure which characterises the nuclei of larger cells cannot be observed. The small size alone would preclude its observation even if it were existent, and possibly may preclude its existence. As it is we may regard some of these scattered granules either as separate nuclei or as the fragments of a single disintegrated nucleus, or, perhaps, of one not yet organised. The rapid motions of some of the cells leads us to suspect the presence of cilia. This surmise is supported by the observation of suitably stained cells. But the demonstration of cilia in this case is one of great difficulty; for not only does their extreme tenuity make them difficult to see, but the bacterial cell exhibits the peculiar idiosyncrasy of casting off its cilia when exposed to any severe shock. Thus it happens that very often the act of killing detaches the cilia, and so when stained no cilia appear. Refined methods, however, have shown that many of the cocci possess a single cilium. Bacilli may be more or less uniformly covered with cilia, they may possess 
a tuft at one end or a single cilium. Similar ciliation may be found in the corkscrew-shaped vibriones and spirilla.

If non-motile bacteria are kept under continuous observation it may be observed that the cells multiply by enlargement and division. Under favourable conditions the interval between one division and the succeeding one may not occupy more than 20 minutes. This appears a moderate rate of reproduction for so small an organism. The very minuteness of the cells favours the prolongation of favourable conditions of nutrition, and it has been estimated, assuming a reproductive division at the end of every 20 minutes in each cell, that a single bacterium would give rise to $16 \times 10^{18}$ individuals in 24 hours. Limited supply of material and the accumulation of waste products naturally prevent the realisation of such a result, as may be easily seen when the weight of such a mass of individuals is worked out. $16 \times 10^{18}$ individuals would weigh between 90 and roo tons. The calculation has its value, however, in showing how quickly an organic medium may become crowded with bacteria.

The consistency of the protein cell-wall varies widely. In some species the wall is a thin membrane which appears to be comparatively hard and smooth. In these forms the products of cell-division do not tend to cohere together, but the resulting individuals easily part from one another either automatically or as the result of external forces. When they retain their original positions but are not bound together in any regular relation the organisms forming such a group are spoken of as staphylococci. In others the products of division cohere and often become bound together by the outer layers of the cell-walls, which are more or less viscid and gelatinous. When the succeeding divisions are parallel to one another the cohering cells form long chains, which, in the case of cocci, are called strepto-cocci. If cohesion is temporary and only lasts till the inauguration of the second subdivision, the cells of a growth or culture appear linked together in pairs. Such forms are described as diplococci. When the succeeding divisions follow at right angles to each other, small cubic bundles or blocks of cells are formed which are called sarcinæ. Again, when divisions follow one another in regular or irregular sequence while the whole culture remains bound together by its gelatinous cell-walls, a gelatinous skin or film is formed which is often described as a zooglœa.

On careful microscopic examination some bacteria show a comparatively large enclosed granule occupying a large or even the greater part of the cavity of the cell. One or two of these bodies 
may be found in the cell. They appear to be formed by the contraction of the protoplasm of the cell from the original wall and round them a second cell-wall is secreted. They are spores. Observation shows that they are often formed when a culture is drying up, or when waste products are accumulating in it. When transferred to a new culture-medium, or when water is added to the desiccated material in which the spores find themselves, these spores germinate by the solution or bursting of the cell-wall and division of the protoplasm. It has been found experimentally that these spores are much more resistant to adverse external conditions than the ordinary vegetative or growing cells. Thus, while, in many instances, the latter are killed by desiccation, spores withstand complete desiccation for months or even years; also, while the temperature of $70^{\circ} \mathrm{C}$. is usually fatal to vegetative cells, spores may resist $100^{\circ} \mathrm{C}$. or even $110^{\circ} \mathrm{C}$. (and if thoroughly dried as much as $130^{\circ} \mathrm{C}$.) and yet be capable of germination.

\section{PRACTICAL WORK.}

Mount a drop of the cloudy salt water which has lain in contact with a piece of red sea-weed for a few days.

With the high power study some of the innumerable specks in the field. Observe both the motionless and the motile ones. Find cocci, bacilli, comma bacilli, vibriones, spirilla, spirochætes, and leptothrix.

Dip a glass rod or needle into the culture and smear a little of the liquid on a clean cover-glass, spreading the liquid uniformly, leaving very little on the cover. Set it apart till quite dry. Drying may be hastened by gentle warming over a flame. When quite dry take the cover in a forceps and, holding the smeared surface uppermost, pass the cover two or three times through a Bunsen flame. This kills the bacterial cells and coagulates the colloids on the outside of the cells, fixing them to the glass. Now place a drop of gentian violet solution on the smear and allow the stain to act for 5 minutes. At the end of this time wash the smear thoroughly under a tap, shake off as much water as possible, and wash with a fine jet of methylated spirit. Again shake off as much as possible and leave the smear to dry thoroughly. When quite dry add a drop of balsam, invert, and place on a slide.

The different forms present in the culture will be fixed, stained, and preserved in this smear-preparation.

It is wise to make a smear-preparation every day after starting the decaying sea-weed. On a certain day the variety of forms will be at its maximum. Previously to that, and subsequently, the less common forms such as spirilla may be hard to find.

Careful examination of these permanent preparations should be made with the high power and the various forms observed should be recorded in sketches. Particular care must be taken not to let balsam come into contact with the high power. Should the front lens, by any accident, become smeared with it, wipe the lens as clean as possible with a clean piece of linen or cotton, and then polish it with a fresh piece moistened with spirit. 


\section{LECTURE VIII.}

A $\mathrm{T}$ first sight you might think it an almost impossible thing to obtain any exact knowledge on the metabolism (the constructive and destructive internal changes) of such minute organisms as bacteria, especially as they are generally found in media of such a complex chemical nature. However, although our knowledge of the physiology of bacteria is far from complete, the researches of bacteriologists have opened for us, by means of their ingenious devices, delicate methods and untiring perseverance, the entry to a store of interesting facts regarding the metabolism of these organisms.

By special methods it has been possible to grow and accumulate sufficiently large masses of bacteria to permit of chemical analysis. In this way it has been found that the same elements enter into their composition as into other plants, viz. carbon, hydrogen, oxygen, nitrogen, sulphur, phosphorus, potassium, magnesium, and calcium. Thus we know that supplies of these elements are needed for their growth. When a mass of bacteria is dried it loses about 85 per cent. of its weight. This loss represents the water of the vacuoles of the cells and that held inbibed in the colloidal substances of the cell-protoplasm and proteins. After drying there remains over about 13 per cent. of the original weight of protein and about I per cent. of fat. And if this total residue is burnt so that all organic substances are oxidised to form carbon dioxide and water, there is left behind a weight of mineral ash which represents sometimes as much as I per cent. of the total weight. These constituents are very similar to those found in other organisms and their relations to one another are in no way exceptional.

The examination of the drop of water taken from contact with the decaying seaweed showed you that there were many kinds of bacteria present which differed from one another in external form. It is possible that they also differ in their metabolism. Furthermore, among those which resemble one another in form, there well may be differences in metabolism. Evidently there is 
but little chance of accurately investigating the metabolism of any one form unless we are able to isolate the different types and investigate them separately. Otherwise it will be impossible to decide whether any product observed is the result of the combined action of a number of different kinds of organisms, or is due to one alone. Hence we find that the first great advances in our knowledge of the metabolism of bacteria were only possible after the pioneers such as Pasteur, Tyndal, and Koch had elaborated methods of isolated culture.

The observation that bacteria grow and multiply most abundantly in organic substances furnished by living or dead plants and animals suggested the use of meat extract as a suitable medium for the cultivation of bacteria. For various reasons which will appear later on it is convenient that the culture medium should be transparent and solid. These two conditions are fulfilled by filtering the meat extract and adding to it sufficient gelatine to make it set at a suitable temperature.

Having thus obtained a suitable medium we are at once confronted with the difficulty that, being suitable to the growth of bacteria, very soon such quantities develop in it that it becomes rapidly decomposed. This difficulty also raises a problem which caused much discussion during the last century, and in its solution evoked a mass of experiment which added greatly to our knowledge and also largely improved bacteriological technique. In this discussion one side maintained that some or all of the bacteria or germs which caused decay and putrefaction originated in the medium by spontaneous generation. The other side held that they were the descendants of spores or cells introduced into the medium from contact with surfaces on which these cells lay, or that they entered into it as dust borne in the air.

It was found that as a general rule when the vessel containing the medium was raised to a high temperature and sealed hermetically the bacteria did not develop and decay was arrested; but, on the other hand, undoubted cases were recorded in which development of bacteria and progressive decay took place in sealed vessels, which had been raised to a temperature of $100^{\circ} \mathrm{C}$. Inasmuch as it was known that protoplasm is killed at a temperature a little over $70^{\circ} \mathrm{C}$. it seemed as if spontaneous generation had occurred, subsequently to the heating, in those vessels in which organisms and decay appeared. The later discovery which showed that spores might withstand a temperature of $100^{\circ} \mathrm{C}$. put another complexion on the matter, and it soon transpired that no development of micro-organisms occurred in those vessels 
in which all the vegetative cells and spores had been killed by the simple ruse described by Tyndal as discontinuous heating.

In this method the vegetative organisms are killed by being exposed to a temperature of $75^{\circ}$ to $100^{\circ} \mathrm{C}$. Then according to observation there are left uninjured only certain spores which will be capable of germination and growth. These are induced to germinate by the restoration of favourable conditions and when they have entered upon their vegetative condition, the temperature is raised once again and catches them in their susceptible condition. Lest the timing of the second heating might have been unfortunate and have found some cells in the resistant state, a second restoration of moderate temperature for some hours and a third heating is arranged. Organic substances thus treated with discontinuous heat and kept free from external infection do not decay, do not produce bacteria nor do they give any indication that spontaneous generation has occurred within them. They are said to be sterilised. And countless such sterilisations made daily attest the absence of proof for such spontaneous generation as was formerly believed to be of common occurrence. The sealing to prevent external infection during and after sterilisation was first effected by using glass vessels and closing the openings by melting the glass. This method has been almost entirely replaced by the use of closely packed plugs of cotton-wool, which are found almost equally effective. They act by filtering out the dust and consequently the dust-borne bacterial cells and spores. The usual apparatus in which the process of discontinuous heat is applied to culture media is the "steamer". It has the form of a cylindrical metal vessel provided with an internal shelf on which the vials containing the culture medium may stand. Below the shelf is water, and the vessel is closed above by a lid with a small aperture to allow the escape of steam generated from the water when the vessel is supported over a burner. When thus arranged the whole vessel becomes filled with steam and the vials containing the medium are quickly raised to the temperature of steam.

Sometimes the "steamer" is nowadays replaced by an "autoclave" which is essentially similar in its design, save that it closes hermetically and is made so strong in the walls that it is possible to generate considerable steam pressure in it without danger. The pressure is regulated by a safety valve. As the pressure of the steam rises, the temperature rises also; in this way the culture medium within is exposed to a temperature which will kill, not only the vegetative cells but also the spores. 
A temperature of $140^{\circ} \mathrm{C}$. is sufficient. By use of the autoclave a single heating suffices to effect sterilisation. It is suitable for the sterilisation of any organic substances which might be charred or injured by a high dry temperature.

The autoclave may also be used to sterilise vessels and instruments used in the isolation experiments spoken of at the beginning of the lecture: but most usually these are sterilised by some form of hot air oven-a metal oven which may be heated by the application of gas burners to a temperature of $150^{\circ}-200^{\circ}$ C., and constructed so as conveniently to hold the vessels and instruments required.

Supposing by means of the processes just described we are provided with a few sterilised test-tubes containing culture medium (meat extract and gelatine) plugged with cotton-wool and a petri dish (to be described later) we may at once address ourselves to attempt the isolation of some of the bacteria in the mixed growth in the seaweed, and proceed as follows:-

Thoroughly liquefy the gelatine medium in a plugged and sterilised test-tube by immersing it in water at about $50^{\circ} \mathrm{C}$. for some minutes.

Loosen the adhesion of the plug of cotton-wool to the inside of the neck of one of the test-tubes, this may easily be done by holding the plug and gently rotating the tube. Set fire to the projecting part of the plug and when the outer surface with any adherent bacteria is burned, extinguish the flame. Take a piece of fine brass wire (24 B.W.G. will do well) somewhat longer than the length of the test-tube. Make a little eye at one end about $3 \mathrm{~mm}$. in diameter. Sterilise this loop and the adjoining few centimetres by heating it in a Bunsen flame; do not let it get red hot. Allow the wire to cool without letting the looped end touch any contaminated surface. When it is quite cool dip it into the mixed growth in the salt water. Still holding the wire between the forefinger and thumb of the right hand, take up the test-tube in the left hand. Pinch the projecting end of the cotton-wool plug between the third and fourth finger of the right hand and by rotating and pulling on the test-tube remove the plug from its neck. Now, still retaining the plug between the third and fourth fingers thrust the wire loop containing the drop of the mixed growth into the culture medium. Withdraw the loop and replace the plug in the tube. Again heat the wire to destroy the adhering bacteria.

If everything has gone well we have some mixed culture enclosed now in the tube and cut off from subsequent contamination. 
At this point we may proceed to separate the individuals of this growth from one another. Place the test tube between the palms of the hands and incline the tube so that it is as nearly horizontal as may be without letting the medium touch the cotton-wool plug. In this position roll the tube to and fro between the hands. The motion will distribute the bacteria through the liquid medium. To secure uniform distribution a considerable amount of rolling must be done. When it is judged that this distribution is effected and the gelatine is still liquid we must transfer it into a petri dish. This latter is a disk-shaped shallow glass dish covered by a similar but slightly larger dish inverted over it. It has been previously sterilised in a hot air oven. Again burn the projecting cotton-wool, pinch the plug of the test tube as before between the right third and fourth fingers and withdraw it. Gently raise the lid of the petri dish, using the finger and thumb of the right hand, and introduce the neck of the test tube over the dish and pour the liquid gelatine into it. Close the petri dish and by suitably inclining it spread the medium uniformly over its surface. Now leave it on a horizontal table to cool and set.

It will be understood that all these operations must be conducted in air as dust-free as possible, so that the chance of contamination while the medium is exposed may be reduced to a minimum. With this in view, draughts and hasty movements which stir up dust should be avoided.

When the gelatine has set in the dish, the latter may be placed aside for a day or so, according to the temperature. If the distribution has been satisfactorily effected, single individuals will be scattered through the layer of the medium in the dish. Conditions being favourable, these cells grow and subdivide, and each becomes the originator of a tiny colony. When multiplication has sufficiently proceeded the colony becomes a visible speck. Where the originating individuals are sufficiently apart each colony is formed of the descendants of one individual and is in fact what may be termed a pure culture. By direct observation we may often notice striking differences between these colonies. The gelatine in the immediate neighbourhood of some is liquid, while around others it is unaltered. The cells of the former colony have produced an enzyme which liquefies gelatine, while the latter do not possess this power. Some of the colonies are pale pink in colour, others are cloudy white or yellow. Differences also in the outlines of the colonies will also be noticed.

In order to examine further these different kinds of bacteria and to keep them free from contamination, a special procedure must 
be adopted. Opening the petri dish cautiously a sterilised piece of wire is stabbed into one of the colonies which we select for further examination, and thus infected the wire is stabbed deep into the still solid medium in a sterile test-tube. Of course, the ritual previously described is strictly observed when opening and closing this tube. It is now set aside for incubation, as the process of growth and reproduction under defined conditions is called.

The growth in the tube may now be submitted to various observations.

Sub-cultures taken from this primary pure culture may also be submitted to various experimental tests, such as culture on various media containing certain substances and omitting others. In this way information as to the nature of the nutrition of the special bacteria under observation may be obtained.

Such observations have proved that the vast majority of bacteria are holozoic in their nutrition, i.e. they depend on organic substances for their supplies of carbon and nitrogen, in contrast to plants containing chlorophyll which draw their supplies of carbon and nitrogen from inorganic substances, as has already been described. This latter type of nutrition is called holophytic, as previously stated. In the case of some bacteria it is found that the organic substance must be supplied by living organisms or it is unfit to act as food. These are parasites and among them are found many which cause disease. Many of these natural parasites can accommodate themselves to live upon dead organic substances and so grade insensibly into the saprophytes which normally subsist on dead organic matter. A very common and almost universally distributed saprophyte is Bacillus vulgaris. It is a long bacillus covered uniformly with cilia and usually develops freely in protein solutions. By its activities and those of its confederates the proteids are gradually broken down into various simpler bodies, e.g. peptones, ptomaines and various aromatic products and finally the inorganic end products of putrefaction, viz. ammonia, free nitrogen, free hydrogen, carbon dioxide, sulphuretted hydrogen, etc. Various bacteria are concerned in these processes and research has not yet delimited the role to be assigned to each. The vast number of saprophytic bacteria, and the complexity of their nutritive substances and their products render the unravelling of the various changes and mutual interactions in the process of decay an investigation of great difficulty.

For putrefactive bacteria proteins can act as a source not only of nitrogen but also of carbon. Culture experiments, however, show that many of them can also utilise simpler compounds to 
provide them with these elements. Thus for example, Bacillus coli thrives when supplied with ammonium chloride and glycerine, and Bacillus subtilis can utilise ammonium tartrate and glycerine, while Bacillus pyocyaneus easily draws its nitrogen from potassium nitrate and its carbon from glucose or glycerine. It has also been found that by varying the source of carbon, the power of utilising various sources of nitrogen may be extended or restricted.

A very remarkable case is presented by the nitrifying organisms (to be described later) which even in absence of light are able to supply themselves with carbon from carbon dioxide by means of the energy supplied in the oxidation of ammonia or nitrous acid.

Again, bacteria are known which are able to assimilate the free nitrogen of the atmosphere.

The elements other than carbon and nitrogen required in the formation of bacterial protoplasm are usually supplied from the water and the soluble salts resulting from the disintegration of proteins or from accidental mineral supplies.

In respiration also bacteria present a large number of variations. Thus some respire free oxygen like the higher plants and animals. These are described as aerobic forms. Others respire combined oxygen and these are the so-called anaerobic bacteria. Sometimes indifferent forms are found which can respire equally aerobically or anaerobically. When a stab infection is introduced into a gelatine medium as in the tube of our cultures, aerobic bacteria develop only at the surface of the medium, anaerobic only in the depths, while the indifferent develop all along the line of the stab. So from the inspection of the growth of a stab infection we can judge whether the bacterium is aerobic, anaerobic or indifferent.

Then again there is much diversity in the substances which are oxidised during respiration. Proteids, carbohydrates and alcohols are commonly utilised. But a great variety of materials seem to be pressed into service and some very remarkable cases are known.

We shall first consider the nitrifying organisms alluded to above.

As has been pointed out, proteins are finally reduced, by the action of various saprophytic bacteria, to ammonium salts, free nitrogen, free hydrogen, carbon dioxide and sulphuretted hydrogen. The ammonia is further oxidised by the respiration of nitrosobacteria to nitrous acid, and the energy set free by this oxidation 
becomes available for these organisms. But nitrous acid is capable of further oxidation and consequently further liberation of energy. This fact is utilised by a second class of nitrifiers, namely, the nitro-bacteria which perform this oxidation and are able to eke out a subsistence by this means. After their respiration the nitrogen is fully oxidised and appears as nitrates. The energy set free in the stages of oxidation of the nitrogen is not only used for the work of the cells such as is usually carried out by respiratory energy, but, as was mentioned before, it enables these organisms to build up organic substances from carbon dioxide and water, effecting thus by means of chemical energy (Chemosynthesis) what green plants are able to achieve by solar energy (Photosynthesis).

Another very interesting example of respiration is afforded by the sulphur bacteria. These organisms are found in any otherwise suitable medium where sulphuretted hydrogen is to be had, e.g. in sulphur springs, or in water where that gas is set free by putrefaction. Their respiration is divisible into two stages. In the first the sulphuretted hydrogen is oxidised, and water and elemental sulphur are produced. Microscopic observation of the sulphur bacteria, which are among the largest known, often shows granules of this sulphur embedded in the protoplasm of the cells. The second stage consists in the oxidation of the sulphur to sulphuric acid. The two processes may be represented in a simplified form by the equations-

$$
\begin{gathered}
2 \mathrm{H}_{2} \mathrm{~S}+\mathrm{O}_{2}=2 \mathrm{H}_{2} \mathrm{O}+2 \mathrm{~S} \\
2 \mathrm{~S}+2 \mathrm{H}_{2} \mathrm{O}+3 \mathrm{O}_{2}=2 \mathrm{H}_{2} \mathrm{SO}_{4}
\end{gathered}
$$

In both stages energy is liberated.

The examples just given illustrate a few points in the nutrition and metabolism of bacteria; however, they must be regarded as representing only in a very limited degree the vast number of different modes of existence which are found in this great group of organisms, which is as yet but very imperfectly explored.

\section{PRACTICAL WORK.}

Apply the method described in the foregoing chapter of making a pure culture of one of the gelatine-liquefying bacteria from the mixed growth in seaweed.

Similarly make a pure culture of one of those which do not liquefy gelatine.

Make stained smear-preparations of several isolated colonies in the petri dish, and examine microscopically. 


\section{LECTURE IX.}

IN the preceding lecture the existence of many kinds of bacteria has been indicated, which differ from one another in external form and ciliation. These constitute the so-called morphological genera. Among those which resemble one another in form there are often great differences in the manner of nutrition and respiration, so that these morphological units must be subdivided into physiological species. It will readily be understood that it is the differences in metabolism which constitute the important distinctions in these forms so far as their relation to mankind is concerned. In this connection may be mentioned the vast number of pathogenic organisms whose adaptations to special supplies of nutritive materials, peculiar waste-products and powers of reproduction are of course far more important than their morphological form.

Then again these same characteristics lend importance to other bacteria which are feared for their destructive action or valued for their useful intervention.

In the destruction of food-stuffs the saprophytes already mentioned have a large share. The many varieties of putrefactive bacteria are concerned in the spoiling of meat, and meat preserving processes are essentially those of sterilisation and the cutting off of the access of bacteria. These same putrefactive bacteria, or some of them, may destroy milk, but the characteristic souring of milk is caused pre-eminently by a special bacterium which produces lactic acid $\left(\mathrm{CH}_{3} \mathrm{CHOH} . \mathrm{COOH}\right)$ from lactose (milk sugar $\mathrm{C}_{12} \mathrm{H}_{22} \mathrm{O}_{11}$ ). The acidity thus developed is unfavourable for the growth of the putrefactive bacteria. Another familiar example of one of these processes depending on the special metabolism of a bacterium is the souring of beer and wine. In this a bacterium participates which oxidises ethyl alcohol $\left(\mathrm{C}_{2} \mathrm{H}_{5} \mathrm{OH}\right)$ and forms acetic acid $\left(\mathrm{CH}_{3} \mathrm{COOH}\right)$.

But certainly one of the most important processes (if not the most important) in which bacterial metabolism plays an essential role is that of the circulation of nitrogen in organic nature. 
It has already been pointed out that all organic nature is indebted to green plants for its supplies of food and energy. All animals depend on them for their nitrogenous food. The green plants themselves obtain nitrogen chiefly in the form of nitrates and to a lesser extent as ammonia salts from the soil-or like Chlamydomonas from the water in which they live. Considering the rate at which plants grow and the quantity of their material which is constantly being used up for the supply of nitrogenous food material for the animal kingdom, we would naturally expect to find large stores of nitrates and ammonia salts in the soil. It is then surprising when it transpires that these substances can as a rule be found only with difficulty in soils supporting luxuriant vegetation. Evidently the supply, which at any one moment is vanishingly small, must constantly be replenished from some continuous source.

As we have seen, putrefactive saprophytes break down complex proteins into simpler ones and finally produce from their disintegration, ammonia, free nitrogen, free hydrogen and sulphuretted hydrogen. These organisms then, seizing on the dead bodies of all animals and plants and their waste-products, pass their nitrogenous substance through their metabolism and give rise to ammonia as a final product. Once this stage is reached putrefactive bacteria can no longer obtain the energy for subsistence. But other bacteria are able to utilise this product as a source of nitrogen and energy. The nitroso-bacteria can oxidise ammonia to nitrous acid and use the energy set free by this oxidation for the assimilation of carbon from carbon dioxide and for the other requirements of life. At the same time some of the nitrogen is assimilated. The third transformation is effected by a third group of bacteria, the nitro-bacteria, which further oxidise the nitrous acid to nitrates. Apparently a large variety of saprophytes participate in the disintegration of the proteins into ammonia, and parasites assist in the earlier stages preparing the way for the true saprophytes. It is remarkable that not only do the nitroso- and nitro-bacteria not require proteins for their metabolism, but even the presence of proteins seems to be poisonous to many species of them ; so that they only develop in quantity when the proteins are disintegrated. As each step in this process has to wait on the progress of the preceding one, an equable and steady supply of nitrates is liberated in the upper layers of the soil. It is from this supply that green vegetation assimilates its nitrogen, building it up into proteins and protoplasm by means of the light energy absorbed from the sun. 
From this short account of the circulation of nitrogen you will realise that every organism in the animal and vegetable kingdoms depends for its existence on the functioning of each link in the complex chain. On the efficiency of each link depends the amount of living matter possible at any one time on the earth. Here I would like to draw your attention to the fact that, as a result of putrefaction, some of the nitrogen of the proteins is set free from the cycle. This constitutes a leak as it were in the system. And so far as it is concerned, it does constantly act so as to reduce the amount of life possible on the earth. Fortunately, however, there are other processes at work restoring the balance and introducing nitrogen into the system. Foremost among these are the activities of a very peculiar group of bacteria. These organisms are found in the soil in the roots of leguminous plants (the group of flowering plants to which the pea, clover and acacia belong). These bacteria enter the roots and there give rise to nodules or tumours, and supplying themselves with energy from the oxidation of the carbohydrates of their host, they are able by some wholly unknown mechanism of their joint metabolism to build up atmospheric nitrogen into proteins. The result is that the supply of nitrates in the soil is reinforced when the host and parasite are ultimately nitrified by the bacteria of the nitrogen cycle, and thus nitrogen is introduced into the system.

We will now turn to the consideration of a wholly different organism-Spirogyra. There are several different species of this plant. One of the commonest is Spirogyra porticalis. They are usually to be found in ponds or very slowly moving water, where they form a mass of green. When taken up into the hand the mass has no tendency to form a mat, but readily separates into numerous filaments of great fineness. This ready separation is due to the nature of the material and to the fact that the filaments are unbranched so that they very easily move over each other and do not tangle together.

For microscopic examination a few of the filaments should be mounted in a drop of water and covered. Inspection even with a low power discloses an organism of extreme beauty. Down each filament of crystalline transparency runs one or more spiral bands of the purest green. Attentive observation shows that, in addition to these bands, each filament carries a series of transverse markings which subdivide it into a succession of uniformly sized portions, and sometimes a darkening and irregularity of the green bands marks the middle of each of these portions. 
With the higher objective Spirogyra reveals further beauty. Careful focussing shows that each filament is made up of cylindrical cells. The first thing that will impress you is the magnificent colour of the bands in the cells. Focussing clearly demonstrates that each band passes round the central cavity of the cell in a spiral path, within and very close to the cell-wall. If the diaphragm of the microscope is partly closed this spiral chloroplast will be seen to be embedded in a thin layer of protoplasm lying against, or lining, the inner surface of the cell-wall. The cell-
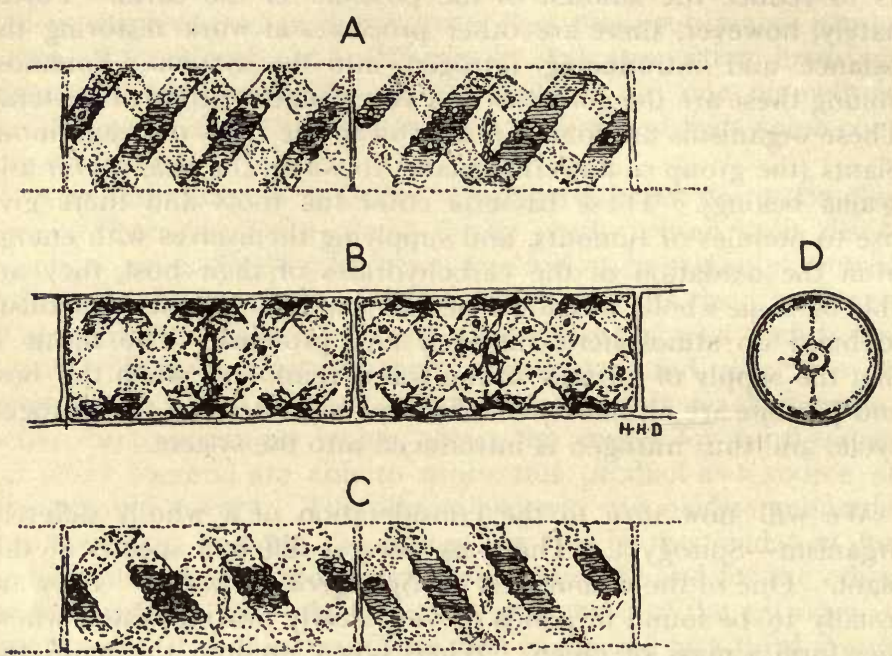

FIG. Ir.-Spirogyra porticalis, $\times 250$. A, focussed on upper side; B, focussed for median plane; C, focussed for lower side; D, reconstructed transverse section.

wall is dazzlingly clear and transparent. It is quite colourless and very thin, as may be seen by focussing for the median plane of the cell. Often the outer surface of the cell-wall is ill defined, the outer layers being less refringent than the inner ones; they evidently form the mucilaginous substance which makes the filaments slippery to the touch. The protoplasm of the cell is also transparent but it is finely granular and occasionally the layer immediately in contact with the cell-wall may be seen in circulation, reminding one of the protoplasm of Elodea. In this case, however, it is only the layer between the chloroplasts and the wall 
which shows this motion. Each chloroplast is ribbon-shaped, slightly grooved on its outer and bowed on its inner surface. There is a thickened rib running down its middle-line. Its margins are irregularly indented. The mid-rib appears thickened at regular intervals into little knobs consisting of a protein mass surrounded with starch grains. These are pyrenoids. By suitable microscopic manipulation it will appear that the chloroplasts are wholly embedded in the cytoplasm and that this latter surrounds a large central vacuole, containing a clear colourless fluid. In the centre of the vacuole one can often plainly see a nucleus suspended. It is a lens-shaped capsule formed by a nuclear membrane enclosing a drop of clear fluid in which is suspended a highly refringent speck, the nucleolus. Generally in healthy cells the axis of the cylindrical cell coincides with the axis of the lenticular nucleus, but a slight mechanical injury or chemical stimulation causes it to rotate so that its axis is at right angles to that of the cell and it presents a circular outline to the observer. It will be noticed that a thin film of cytoplasm adheres to the outer surface of the nuclear membrane and from the capsule so formed enclosing the nucleus very fine filaments of cytoplasm extend and connect the capsule with the cytoplasmic lining of the cell-wall. It will be noticed that most of these filaments may be traced to the cytoplasm in the immediate neighbourhood of a pyrenoid.

So far as is known the metabolism of a cell of Spirogyra is very similar to that of Chlamydomonas. When exposed to light and when the water which surrounds it is charged with carbon dioxide, as is normally the case, the evolution of oxygen bubbles shows that photosynthesis is being carried on. During this time suitable observations show that the starch in the pyrenoids increases. Spirogyra lives in water that is free from organic nitrogenous compounds and it evidently obtains its nitrogen from the traces of dissolved nitrates in the water surrounding it. Hence its nutrition with regard to carbon and nitrogen is typically holophytic.

Its respiration is aerobic and if the water in which it lives is deprived of dissolved oxygen, Spirogyra dies.

Possessing a complete cell-wall Spirogyra has no contractile vacuole and hence no special apparatus which eliminates dissolved substances including waste-products. Such active elimination seems rendered unnecessary by the very economical metabolism which Spirogyra possesses in common with the majority of the vegetable kingdom. Thus the waste-products of its catabolism, both nitrogenous and carbonaceous, act as the raw materials of 
anabolism for the assimilation of nitrogen and for photosynthesis. Hence excretion becomes unnecessary.

The accumulation of assimilated material is accommodated by the growth and elongation of the cell. After continued elongation the nucleus divides. 'The two resulting nuclei remain connected together by a barrel-shaped mass of cytoplasm occupying the middle of the cell. At the same time an annular deposit of cellulose may be observed forming on the inside of the cylindrical surface of the cell-wall about midway between the two end walls. This annular deposit increases until it forms a diaphragm partially dividing the original cell into two. The barrel-shaped mass connecting the nuclei is ultimately encroached upon by the gradual closing of the perforation in the diaphragm and the chloroplasts are also cut in two by it. Finally the perforation closes entirely and divides the original cell into two separate compartments each having its own nucleus which now draws away from the partition and occupies the middle of the new cell. The new cell may now grow and the process be repeated. It is observed that cell and nuclear division usually take place during the night or early hours of the morning: but when once started they may be delayed by exposure to a low temperature.

Growth and cell division may take place in any cell and lead to the rapid lengthening of the filaments. The adjoining cells of the filaments are very slenderly attached to each other so that the lengthened filaments easily break into parts. Thus multiplication and reproduction is effected. This method of reproduction is very prolific during the growing period of the cell, viz. in spring and summer.

Besides this asexual method, Spirogyra has a sexual method of reproduction, to which it appears to resort at the end of a period of vigorous growth. At this stage Spirogyra takes on a very different appearance and also feels different to the touch. It loses its brilliant colour and becomes gradually pale or even brownish. Its filaments meet together and form a tangled mass. The process, which is called conjugation, begins by each of the opposite cells of two adjacent filaments pushing out a slight prominence on the side nearest its opposite neighbour. The protoplasm of the cells extends out into these outgrowths which become tubular in form and are each directed toward the similar outgrowth from the opposite cell. After close contact has been established between the opposite pairs, the walls covering the ends of the outgrowths are dissolved and the contents of the two cells come into union with one another through this "con- 
jugation-tube," as it is called. As soon as the two masses of protoplasm run together in the conjugation-tube the protoplasm remaining in the cells begins to draw away from the cell-walls, apparently by a reduction in the size of the vacuoles. The retraction is much more marked in one of the pair than in the other, and finally the protoplasm evacuates it entirely and is drawn over and passes through the conjugation-tube into the other cell. In this cell the total protoplasm of the two cells, cytoplasm, nuclei and chloroplasts, fuses to form an egg-shaped mass. A strong cell-wall is secreted round this compound mass, or zygospore as it is called, and a period of rest is entered upon, during which the zygospore often remains enclosed in

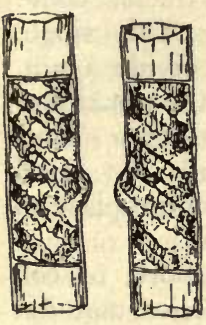

A

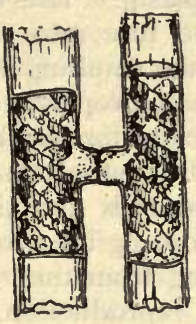

B

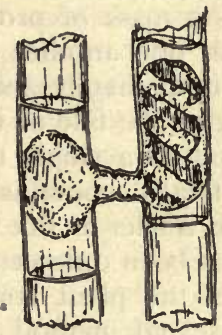

C

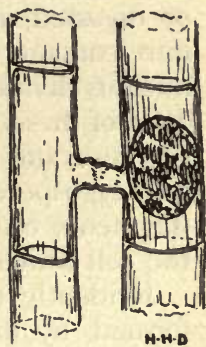

D

FIG. 12.-Spirogyra porticalis, conjugating cells, $\times$ 125. A, beginning of the formation of the conjugation processes; $\mathrm{B}$, processes in contact; C, conjugation-tube completed by solution of end-wall of processes, fusion of the two cells effected, male cell is seen passing into the female cell; D, zygospore lying in the female cell-wall.

the original wall of the conjugating-cell. At the end of its rest the outer layer of the zygospore's wall gelifies at one point and a tubular extension of the inner layer is thrust out, thus converting the egg-shaped zygospore into a more nearly cylindrical cell. The protoplasm within thus obtains space to distend itself and the chloroplast to unravel till the zygospore gradually as, sumes the appearance of an adult cell of Spirogyra. As it elongates it ruptures the enclosing cell-wall of the original conjugating-cell. At first one end of the young plant, or embryo, is very often narrower than the other and into it the chloroplast does not extend. This narrow, colourless end or process takes on the function of a sucker and attaches the embryo to solid objects. Soon, however, cell-division produces a filament 
from the embryo, and fracture separates the plant from this transitory organ of attachment.

There are many points of interest raised in this sexual reproduction of Spirogyra. In the first place, what happens within the mass formed by the two uniting cells? Direct observation gives us no clue. The mass is so dense and contracted that the behaviour of the chloroplasts, much less that of the nuclei, cannot be followed. Investigators, however, by using very refined methods of killing conjugating cells at various stages of the process and by using stains which colour differentially the various parts of the cells have been able to give us an account of these mysteries. The chloroplast of the cell which moves across the conjugation-tube disappears-apparently dissolved or digested in the mass of protoplasm. The two nuclei come into contact with one another and fuse into one. This single nucleus divides immediately and the resulting two divide again. Two of these suffer the fate of the chloroplast and the remaining two fuse and produce a single nucleus for the zygospore. Thus the zygospore finally contains the cytoplasm and the fused fragments of the nuclei of the two cells and the chloroplast of the cell which has been quiescent during the process.

Evidently then the plant coming from the zygospore, or cell formed by the sexual method of reproduction, carries material derived from two individuals. This may affect the offspring in two opposite ways. It has a two-fold possibility of inheriting idiosyncrasies; at the same time the idiosyncrasies of one parent will tend to be nullified by those of the other. These differences between sexual and asexual reproduction should always be borne in mind.

In the conjugation of Spirogyra two complete individuals fuse-the whole individual acting as a sexual cell and passing complete into the zygospore, where its activity re-awakens in the next generation. The process is apparently repeated indefinitely so that, apart from accidents, we may regard the protoplasm of Spirogyra or Chlamydomonas (which in this respect is similar) as immortal. It simply undergoes subdivision and fusion.

These two organisms further resemble each other in the fact that the two cells which fuse in sexual reproduction are, externally at least, similar to one another. It has been pointed out that in the process of conjugation in Spirogyra one of the fusing cells-gametes-exhibits more motion than the other and is consequently often spoken of as the sperm or male cell, the 
quiescent cell being regarded as the female gamete or ovum. However, before the process begins, the two are not visibly differentiated. It will often be noted that all or most of the cells of one filament behave as sperms in relation to an adjoining filament. Thus we may say that the filaments are male and female. In certain species of Chlamydomonas the gametes are of different sizes and the larger are supposed to be the ova.

Where there is a marked differentiation between the sexual cells the fusion process is usually spoken of as fertilisation.

Another point naturally arouses speculation when the conjugation of Spirogyra is observed. What can evoke the outgrowth of the conjugation-tubes? How are they directed towards one another? It would be highly unsatisfactory to attribute this growth to some mysterious telepathy, action at a distance. As was previously pointed out our knowledge of the ultimate structure of protoplasm and of the transformations of energy which take place within it, is so vague that in these cases of so-called vital action we cannot hope to see the direct connection of cause and effect; but at any rate we may look for the stimuli which evoke certain responses. The cells, so far as we know, have no visual organs. In this case the stimulus is very possibly a chemical one. The adjacent cells may liberate some substance into the water which is perceived by both or they may, as it has been suggested, deprive the surrounding water of something, e.g. carbon dioxide, and the absence of this acting as a stimulus may evoke as response the growth of the conjugation-tubes. If this last surmise is founded on fact we would have another instance of starvation acting as a stimulus in sexual reproduction.

Again, it is conceivable that the production of the conjugationtubes is the direct result of the development of a cellulose softening enzyme which requires the addition of a certain concentration of a soluble diffusable substance (co-enzyme) to make it efficient. This co-enzyme may be supposed to diffuse out in all directions but only attains sufficient concentration between the filaments. It is therefore only on the adjacent aspects of the cell-walls that sufficient softening occurs to secure the yielding of the wall to the internal osmotic pressure. The first beginnings of the conjugation-tubes are the result of this yielding. The subsequent direction of growth of the prominence (or bulge) thus produced is determined by the region of greatest concentration which will evidently be towards the corresponding outgrowth of the opposite cell. The observed fact that a current 
of water is inimical to the production of conjugation-tubes supports this view.

\section{PRACTICAL WORK.}

Mount a few filaments of Spirogyra and cover.

First examine and sketch with low power. Estimate the dimensions of the cells by means of the Ghost-micrometer.

Examine with the high power. Notice the course of the spiral chloroplasts by use of the fine adjustment. Identify the cell-wall and focus particularly to observe its thickness. By closing the diaphragm observe the layer of cytoplasm in which the chloroplast is embedded. Look for motion in its outer layer. Observe the serrated margin of the chloroplast, its mid-rib and pyrenoids. Focus for the nucleus and observe its membrane and nucleolus. Notice the capsule of cytoplasm covering over its surface and the filaments extending from it to the layer of cytoplasm lining the wall. Trace these filaments by means of focussing from the capsule to their outer extremities.

Make three drawings of the cell as seen at three different levels: (I) Focus for an optical plane or section cutting the cell just within the near curved surface of the cell-wall and tangential to the nearer turns of the chloroplast. Fill in all the details in your sketch which you can observe without changing the focus. This upper tangential section should show a continuous sheet of cytoplasm lying inside the part of the cell-wall nearest the observer and in this sheet those portions of the chloroplast which are approximately in the same plane. These appear as oblique straps in the field, their ends passing out of focus. As you focus downwards (2) the median optical section comes into view, and the near parts become hazy and disappear. The cell-wall appears as a very narrow refringent band outlining the whole cell. The cytoplasm is within this and is much less plainly delimited. The chloroplast appears as a number of isolated crescentic green patches in the cytoplasm. The hollows of the crescents are turned towards the cell-wall. Centrally may be seen the nucleus-its membrane and nucleolus. Usually a tuft of cytoplasmic filaments takes origin from the cytoplasmic capsule of the nucleus, where the latter is closest to the peripheral cytoplasm. Focus still deeper and you will see (3) the further tangential optical section. It resembles in all respects the nearer one, save that the slope of the portions of the chloroplasts in focus is in the opposite direction.

From the consideration of these three sketches reconstruct a transverse, section of the cell passing through the nucleus. 
THE types which we have examined so far, Chlamydomonas, Saccharomyces and Spirogyra, are unicellular plants. That is, each cell is a complete plant and discharges all the functions of life. It assimilates, respires, frees itself from waste-products, grows and finally reproduces itself. In Chlamydomonas the cells break apart as soon as they are formed and pursue a separate existence so that there can be no question that each separate individual consists of a single cell. The same appears true of Saccharomyces, in which as soon as the budded cells have attained a mature size-or even earlier-they are so easily separated from one another that under normal conditions each becomes independent and carries on a separate existence. But it was also seen that under special conditions-for example, in the gelatine culture-the separate cells remained together and formed a branching system which might remind one of the higher plants. However, this coherence is purely accidental and the individual cells, notwithstanding their proximity to one another, are each complete individuals and discharge all the functions of an individual. The accidental coherence of the individuals of Saccharomyces naturally leads us to the case of Spirogyra. Here coherence is more than accidental and is the general rule. But notwithstanding this fact, the separate cells of Spirogyra are rightly regarded as individuals, and the filament as a colony. Each carries on its own life-processes independently of the rest. In the manner of carrying on these processes they are not influenced by their neighbours, and the protoplasm of each cell is completely isolated from the rest by its cell-wall. Only in their sexual reproduction do they show any differentiation, and that differentiation, such as it is, is a differentiation of individuals which in this case are unicellular. Such unicellular individuals, which not only discharge all the vegetative functions but also give rise by division to new individuals, are, as has been pointed out, putting the chance of accident on one side, immortal.

In this lecture we come to the study of an organism which 
crosses the border line separating unicellular and multicellular plants.

Volvox is found in fresh water, very often in the pools or ditches of woods and forests. It has the form of a transparent, gelatinous sphere approaching or even attaining half a millimeter in diameter. It is of a diaphanous green colour. There are few who would not be impressed by the unique beauty of its appearance. Microscopic examination shows us that in the healthy condition the spheres of Volvox are motile, and that while they steadily advance through the water in one direction, with one pole constantly pointing forwards, they also rotate with a stately motion round that pole in the line of their advance. Their rate of progression is about I $\mathrm{mm}$. per second. It also is seen that the

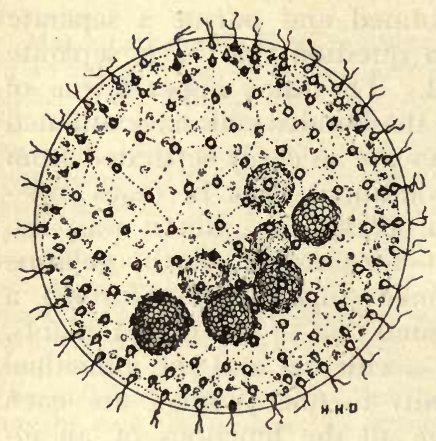

FIG. 13.-Volvox aureus, asexual sphere, $\times 80$. Young spheres are seen within the parent. green colour of the sphere is due to countless specks (they vary in number from 200 to $50,000)$ situated near the surface of the sphere and embedded in a colourless, hyaline material. A few larger green bodies may be seen in many of the spheres. Focussing and manipulation of the light shows that the central region of the sphere is clear and apparently filled with water.

With a high power and repeated manipulation of the light the outer surface of the hyaline jelly which forms the wall of the sphere may be made out and it will be noted that while the transition of the jelly outwards into the water is abrupt and a defined surface is visible, on the inner side this transition is gradual and the jelly passes insensibly into the watery space within. The green bodies embedded in the jelly, seen as specks with the low power, are now easily made out to be cells. Each is a tiny pear-shaped mass of protoplasm (say about $8 \mu$ long) with its narrow end just reaching the outer surface of the jelly. The green colour is conferred on each by the presence of green pigment located in the inner end of the cell. The pigment is associated with a bell or cupshaped chloroplast like that of Chlamydomonas. In the substance of the thickened, inner and closed end of the chloroplast a 
pyrenoid may usually be seen, and a nucleus lies in a central mass of protoplasm filling the hollow of the chloroplast. The tapered end of the cell is colourless like the beak of Chlamydomonas, and in this part may be seen, under favourable conditions, two contractile vacuoles. Further, the similarity between these cells and Chlamydomonas is strengthened by the possession of two cilia which extend from the outer end of the cell into the surrounding water. It is the vibrations of the cilia of all the cells which confer motility on the sphere. An eye spot or stigma like that of Chlamydomonas is found in those cells of the sphere which lie round the anterior pole. On the whole we may regard Volvox as an aggregation of cells similar to the individuals of Chlamydomonas. The cell-walls of the cells have become gelatinous and fusing together form the hyaline wall of the sphere. Using proper fixing (or killing) fluids and suitable stains it is seen that a well-defined pellicle sharply delimits and covers the swollen cell-walls at their outer surface and is reflected radially inwards along their surfaces of mutual contact. The same treatment establishes the fact that their internal walls gradually merge into the central watery cavity.

But although the limits of these gregarious cells are thus sharply defined towards one another by the outer layer of their cell-walls, careful observation of a tangential optical section shows that each mass of protoplasm (protoplast) is connected with its adjacent fellows by extremely fine fibrillæ of protoplasm. These form a network throughout the wall of the sphere, each protoplast or cell occupying a nodal point of the meshes. Hence it is that all the protoplasts of the sphere are directly connected with one another and the protoplasm of the sphere is continuous throughout. Generally these fibrillæ are single; sometimes, however, several parallel fibrillæ, connect adjacent cells.

In our preliminary observations the steady progress of the spheres through the water was noticed. A few trials, by turning round the preparation and cutting off direct light, will convince an observer that this motion is a response to the stimulus of light, in this resembling the motion of Chlamydomonas. It is evident that to direct the motion of Chlamydomonas the co-ordinated motion of its two cilia is necessary and presumably the stimulus received by the stigma is transmitted to the protoplasm at the base of the cilia and there liberates energy to activate the cilia. The motions of these cilia must be co-ordinated with the direction of the light so that they exercise a turning force on the cell unless its axis points along the path of the illuminating beam. In 
Volvox evidently, the co-ordination must be of a more complicated nature, for while the cilia of the cells immediately at the anterior pole may act in a way comparable to a single cell of Chlamydomonas, those at the equator and at other parts of the sphere must react differently to the light, and in order to produce the proper propulsive and orienting forces which make the sphere move towards the source, stimuli from the cells bearing stigmata must be conveyed to every other cell and the responses must be so harmonised and co-ordinated that the observed steady motion will be produced. Probably the elaborate network of protoplasmic fibrillæ finds one of its functions in transmitting these co-ordinating stimuli.

Just as in the case of Chlamydomonas, a moderate light stimulus attracts Volvox. An intense beam of light, on the other hand, repels it. When cut off from light the sphere wanders about exhibiting a so-called spontaneity in its motions. This spontaneity is probably to be attributed to infinitesimal stimuli, often of a chemical nature, or its actions may be determined in part by the previous history of its various constituents acting like the hysteresis of colloids and producing effects which are often spoken of as physiological memory.

So far as is known the nutrition of Volvox resembles that of Chlamydomonas. It obtains carbon by photosynthesis. The evolution of oxygen and the growth of starch in the pyrenoids tells of this process. Also its acquisition of nitrogen is from nitrates. It is in fact a holophyte. Simultaneously with its constructive processes, destructive changes are taking place in its cells. The chief of these is respiration and its waste products, carbon dioxide and water are eliminated, while free oxygen is absorbed. It is this aerobic respiration or oxidation of the carbon assimilated in photosynthesis which provides the energy utilised in ciliary motion and other vital processes. Other waste products not so easily defined are eliminated by the action of the contractile vacuoles.

Volvox has both sexual and asexual reproduction.

In most spheres of Volvox a number of smaller spheres are seen enclosed. There are usually about eight, and they are disposed round the posterior pole within the sphere. These enclosed bodies vary in appearance according to the stage of their development. In their earliest stage they are enlarged spherical cells which resemble the other cells of the sphere, but owing to their size protrude from the wall into the internal cavity of the sphere. The protoplasm of each of these cells contains an 
irregular chloroplast of large dimensions which confers a green colour on the cell. The subsequent history of each of these cells is as follows: the contents of the cell divide within its cellwall into four protoplasmic masses which do not entirely fill the original wall but are aggregated round one of its poles. Each of these four masses or cells now divide and eight cells are produced which flatten themselves against the inside of the original cellwall so as to form a saucer-shaped mass. Growth accompanied by repeated divisions and readjustments among these cells forming the saucer finally convert it into a hollow sphere with a pore leading into it. This pore occupies the pole opposite the centre of the original saucer-shaped group. During these developments the spherical group, now recognisable as an embryo, projects into the cavity of the parent Volvox, as do those other metamorphosed cells in which the same processes have taken place. The cells in the walls of these embryos multiply and their cell-walls swell and become gelatinous.

Finally the development of cilia from its outer aspect converts the embryo into a miniature sphere of Volvox rotating within the cavity of its parent. The embryo then escapes with its fellows by the disruption and death of its parent. As it escapes the enlarged cells which are to form the coming generation may be plainly distinguished among the vegetative ciliated cells which form its wall. In fact, before the embryos escape, we may often see stages in the development of three generations through the transparent walls of the parents. In many spheres a clear cap, devoid of cells and their protoplasmic connections, can be seen occupying the posterior pole. This appears to be the persisting remnant of the opening left by the incurving margin of the saucer-shaped embryo. During subsequent development it has become closed by the gelatinous material of the adjacent cell-walls. The cells provided with stigmata are the direct descendants of the central cells of the embryo, while the reproductive cells are descended from some of the peripheral cells. When the embryo assumes the spherical form the anterior region may be described as the light sensitive hemisphere and the posterior region round the pore as the reproductive region. The cells giving rise to these embryos, inasmuch as they develop into new individuals without fertilisation, may be called spores.

Sexual reproduction is usually found in Volvox in spheres distinct from those which carry on the production of spores. The sexual individuals produce two kinds of gametes or sexual cells; sometimes both are formed in the same and sometimes in different 
individuals. The origin of both ova and sperms is similar to that of the spores. Certain cells in the wall of the young individual
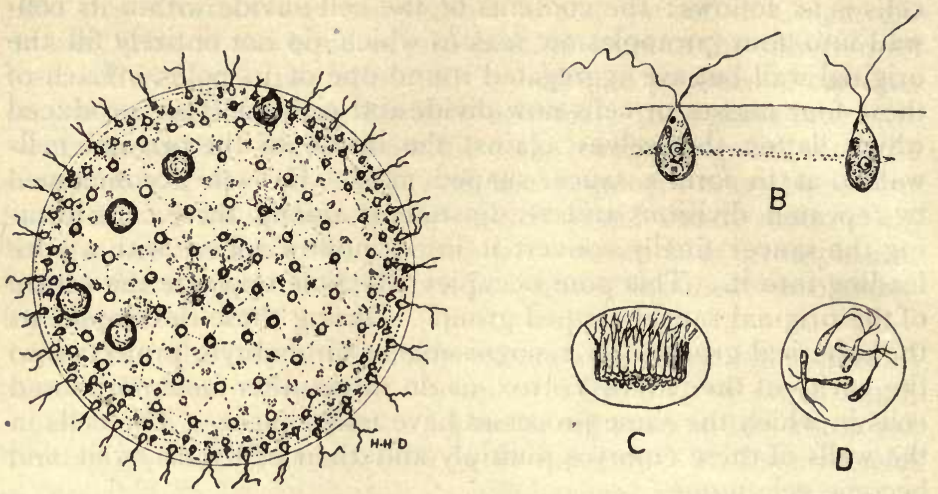

\section{A}

Fig. I4.-Volvox aureus. A, female sphere, $\times 80$, showing nine ova; $\mathrm{B}$, two adjacent somatic cells with protoplasmic connections; C, antheridium, with packet of sperms; D, sperms having broken loose from packet.

enlarge. In the case of the ova this enlargement is accompanied by a darkening or intensification of the green colour. This

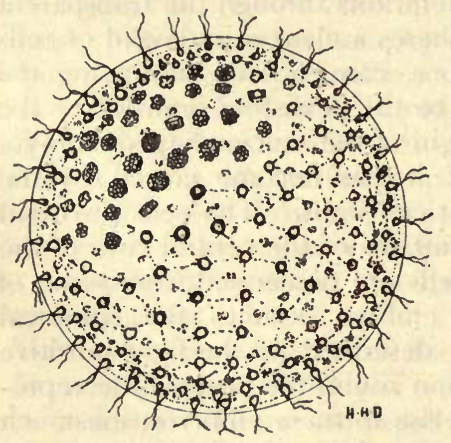

FIG. I5. - Volvox aureus, male sphere, $\times 80$, showing packets of sperms in the antheridia. enlargement and darkening are the only marked visible changes undergone by the cells which are to become ova. In this condition, enclosed in a cavity of the cell-wall, they await fertilisation. The enlarged cells which give rise to the sperms are lighter in colour. While still enclosed in a cavity in the wall of the sphere, they subdivide and produce a plate of cells. The cells of this plate further subdivide, always however at right angles to the surface of the plate, and thus form a large number of narrow prismatic cells (32) lying parallel to each other like the faggots in a bundle. These cells gradually become tapered towards one end 
while the other remains blunt. Two cilia develop from the tapered end. The movements of these cilia at first move the plate as a whole; later on they shake the cells free from one another and the latter now swim about in the wall-cavity as elongated separate cells - the sperms. Finally they emerge from their cavity and swim away to an ovum-carrying individual and penetrating through the wall or entering by the pore may each fuse with and fertilise an ovum. Sometimes the plate of immature cells does not disintegrate into separate cells until it has penetrated into the female individual carrying the ova.

The sperms are very small (about $10 \mu \times 3 \mu$ ), pear-shaped masses of protoplasm. Each has a nucleus, a yellow-green chromatophore with pyrenoid, a stigma, and two contractile vacuoles. The two cilia are borne on their tapering ends. The ova are much larger, being about $5 \circ \mu$ in diameter. They have a large nucleus, and their dark green chloroplast has many pyrenoids.

The zygote or cell formed by the union of ovum and sperm develops round itself a hard cell-wall furnished with warty prominences. After a period of rest which coincides with the duration of winter, the outer coat is dispensed with and the contents develop, in the same way as one of the spores, into a new Volvox.

When we reflect on the structure of Volvox, how every cell is intimately bound together, being in fact just a portion of one coherent mass of protoplasm, with a community of nutrition and other physiological processes throughout, how stimuli and coordinating messages are sent from one part of the organism to another, and how the organism responds to these stimuli as a whole, the conviction establishes itself that in Volvox we have to do with an individual. This conviction is rendered more certain when observation shows that Volvox reproduces itself as a whole. In its life history special reproductive cells are set apart, and from these, both new reproductive cells and those which are not concerned with reproduction-namely somatic cells-are formed. Hence we have good reason to regard Volvox as a multicellular individual.

The salient feature to which we must now direct our attention is the fact that the organism is differentiated into reproductive and non-reproductive cells (germ cells and somatic cells respectively). It is only the protoplasm of the reproductive cells which continues to live from one generation to another. They form the immortal part of the species. From the moment in 
which the somatic cells are differentiated they are cut off from this immortality. Hence with the differentiation of an individual into germ cells and somatic cells mortality is introduced into the species; and as this differentiation is only possible in multicellular organisms we find that mortality is introduced with the transition from the unicellular to the multicellular state.

From this point of view we may regard the germ cells of any multicellular organism as continuous lines of living protoplasm producing at regular intervals excrescences by cell-division. The individuals are these excrescences developed periodically on the lines which, it may be, extend through geological epochs. In unicellular organisms these periodic excrescences are not developed.

\section{PRACTICAL WORK.}

Examine spheres of Volvox in a drop of water uncovered. Observe the direction of the motion of the free-swimming individuals. Reverse the slide on the stage so that the side which was nearest the window is now furthest. Again observe the motion. Cut off the direct light and observe the organism by means of the light transmitted from the mirror through the condenser alone.

Before applying the high power a cover-glass must be put on. To prevent the latter crushing the Volvox put a few fibres of cotton-wool in the drop before laying on the cover-glass. With the high power focussed on the side of the sphere turned towards the observer, it should be possible to make out the individual cells as seen from in front, also their protoplasmic connections, the chloroplast, pyrenoid and nucleus. If we are observing the anterior pole, stigmata or eye-spots should be seen in each of the cells. It often requires very careful focussing and adjustment of the light before these structures can be made out; sometimes by focussing on the outer surface of the sphere the cilia may be seen.

The same structures should be looked for in the limb or profile of the sphere. From this aspect we see a side-view of the cells. The colourless beaks coming to the surface of the sphere should be noted and the cilia projecting from them looked for. Also this profile-view shows the chloroplast, pyrenoid and nucleus more easily than the previous one. The stigma may also be found, but the protoplasmic connections are difficult to make out.

A sphere mounted in liquor iodi shows the cilia and protoplasmic connections plainly.

To observe the pellicle which coats the outer and radial cell-walls spheres fixed in 4 per cent. formalin and stained with watery gentian violet are suitable. 


\section{LECTURE XI.}

VAUCHERIA is a very common plant. It may be found on the surface of moist ground, on stones submerged in streams, or on rocks splashed by trickling water. Some kinds also are marine. It forms a mat-like growth of fibrous green filaments which are tough, and rough to the touch, and are tangled together owing to their branching. The colour is dark green. The branching fibres may be several centimetres in length.

Owing to their matted state it is hard to separate a portion for microscopic examination without injury to it. Supposing we are able to obtain a satisfactory specimen, it will be easy to see that each fibre is tubular in form, about $80 \mu$ in diameter. The fibres form many branches, some of which are colourless and of a curiously irregular form. These latter are rhizoids, which fix the plant in position by adhering to sand particles, etc. The rest of the plant has the dark green colour. The whole plant is covered by a continuous membrane. It is of cellulose and is spoken of as a

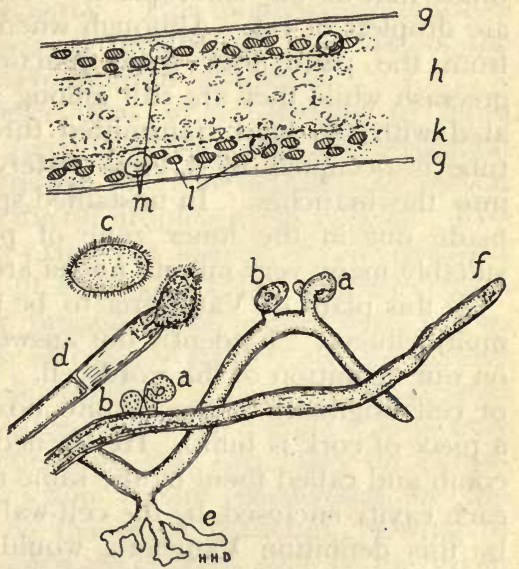

FIG. I6.-Vaucheria sessilis, $a \cdot f, \times 40$. $a$, antheridia ; $b$, oogonia ; $c$, zoospore; $d$, partition cutting off zoosporangium from the rest of coenocyte; $\varepsilon$, rhizoids ; $f$, growing tip of coenocyte; $g-m$, above, optical section of coenocyte, $\times$ 30o. $g$, cell-wall; $h$, vacuole; $k$, layer of cytoplasm; $l$, chloroplasts; $m$, oil-drops.

cell-wall. It has the usual characteristics of this substance, but is rather thicker than the cell-walls of the plants which we have hitherto examined. The green coloration is located in a layer of material lying against the inside of this branching 
cellulose tube. Closer examination shows that the green colour is actually confined to very minute ovoid or lens-shaped chloroplasts embedded in a comparatively thick layer of protoplasm, which forms a lining to the cell-wall. 'This lining extends throughout the whole plant and out into the branches, where it thickens and completely fills their extreme ends. The chloroplasts are limited to the outer zone of this layer and are most closely packed in the ends of the branches. There are no chloroplasts in the protoplasm of the rhizoids. The chloroplasts are very small and appear quite homogeneous, having no pyrenoids. In the same zone and intermingled with the chloroplasts one sees considerable numbers of bright globules varying in size from very minute specks up to ones which have a diameter twice or three times that of the chloroplasts. Chemical tests show that these are droplets of oil. Although when they are artificially liberated from the plant they seem practically colourless, they appear greenish while they are still among the chloroplasts and illuminated with the light transmitted through them. The axis of the tube is occupied by a large watery vacuole which extends out into the branches. In unstained specimens no structures can be made out in the inner zone of protoplasm but when stained suitably many very minute nuclei are seen in it.

Is this plant of Vaucheria to be regarded as a single cell or as multicellular? Evidently our answer to this question will depend on our definition of the word cell. Robert Hook gave the name of cell originally to the minute box-like chambers out of which a piece of cork is built. He likened them to the cells of a honeycomb and called them by the same name. Evidently he regarded each cavity enclosed by the cell-wall as a single cell, and judged by this definition Vaucheria would be a unicellular plant. On the other hand, it is now well known that Hook only saw the dead remnants of cells in the cork, which when alive contained, like the vast majority of cell-walls, a mass of protoplasm. This mass in its living state is differentiated into nucleus and cytoplasm. The cell-walls are formed by the activities of the protoplasm and many instances are known in which these masses of cytoplasm with a nucleus remain for the whole, or part, of their lives without a cell-wall. Hence the wall has come to be regarded as an unessential part of the cell, the essential parts being the nucleus and cytoplasm. You have already seen in the multicellular Volvox that although the cells forming the sphere are each enclosed in their cell-walls, yet the cytoplasm of all the cells is continuous, by means of the protoplasmic connections, through- 
out the whole organism. In Vaucheria we find numerous nuclei indicating the organic centres of as many cells but the continuity of the cytoplasm of all the cells being merged in an unbroken film is more complete than in Volvox. Such structures as this, in which many cells are fused, the cytoplasm being continuous and the whole covered by a common cell-wall, are called cœnocytes.

It may be appropriately recalled here that investigation has shown that the cells of bacteria do not possess a characteristic nucleus, so that we have again to revise our conception of a cell and we may define it as a more or less isolated and independent mass of protoplasm usually differentiated into a nucleus and cytoplasm and often possessing a cell-wall.

A search by means of suitable reagents will soon convince one that starch is absent from Vaucheria. This immediately indicates that a difference exists between its metabolism and that of the other green plants which we have hitherto examined.

It will be observed, however, that when exposed to light, oxygen is evolved from the green branches and this evolution does not occur if the surrounding water is deprived of carbon dioxide. During this process the oil droplets grow in bulk. Evidently photosynthesis is taking place, but instead of a production of starch going on as a result of this, oil is being formed. Inasmuch as oils possess a smaller proportion of oxygen than carbohydrates, evidently the volume of oxygen evolved will be greater than that of the carbon dioxide absorbed, in place of being equal to it as is the case when a carbohydrate is formed. It is only protoplasm furnished with chlorophyll that can carry on photosynthesis and hence this function is not discharged by the colourless rhizoids but only by the green branches. Owing to the position of the rhizoids, which are covered over by the green branches and embedded between the particles of sand and grit, they are cut off from the light and so, even if they possessed chlorophyll, could not carry on photosynthesis. Hence they have come to act as organs of attachment for the plant, while the function of photosynthesis is assigned to the rest. There is thus a division of labour between the parts of this organism. The absorption of nitrates, so far as we are aware, may be carried on all over the surface of the cœnocyte.

No excretory organs of any kind are known. As has been pointed out, the presence of a continuous cell-wall precludes the development of a contractile vacuole. Also the working up again into new protoplasm of any nitrogenous waste produced seems a characteristic of vegetable metabolism. Carbon dioxide is, 
however, produced and may be evolved if the plant is not exposed to light. This gas escapes in solution from the surface of the protoplasm and diffuses away in the water. The production of carbon dioxide is the result of respiration, which in the case of Vaucheria requires dissolved free oxygen. In other words, Vaucheria's respiration is aerobic. It has been pointed out that when carbohydrates are oxidised in respiration the volume of oxygen employed is equal to the volume of carbon dioxide produced. This appears from the equation: $\mathrm{C}_{6} \mathrm{H}_{12} \mathrm{O}_{6}+6 \mathrm{O}_{2}=6 \mathrm{CO}_{2}+6 \mathrm{H}_{2} \mathrm{O}$ and is usually expressed by saying the respiratory ratio is equal to one $\left(\mathrm{CO}_{2}: \mathrm{O}_{2}=\mathrm{I}\right)$. It is evident, however, that when a plant respires oil like Vaucheria, it oxidises a substance poorer in oxygen than those which respire carbohydrates, consequently the respiratory ratio in the former is less than unity. Therefore oil is a more concentrated form of store material as a supply of energy than starch or sugars.

When conditions continue favourable for photosynthesis and assimilation of nitrogen, the synthetic or anabolic processes predominate over the wasting or catabolic processes, and a surplus of organic substance is accumulated. This is available for growth and also for the repair of injury.

If a filament of Vaucheria is wounded the living protoplasm retracts itself a short distance into the tubular wall of the cœnocyte from the point of injury. At the same time the retreating end secretes a covering of cellulose and makes this new portion continuous with the old cell-wall, thus isolating itself from the injured spot. The transverse walls thus formed in response to injury are the only transverse septa found in the cœenocytes of Vaucheria, except those formed in connection with the reproductive organs which will be mentioned later.

The growth of Vaucheria takes place at the tips of the branches. As was before noticed the ends of the branches are filled with plug-like masses of protoplasm closely packed with chloroplasts. Stained specimens show that the minute nuclei here are subdividing rapidly. The wall covering the rounded end of the branch is somewhat thinner than elsewhere and being gelatinous is more ductile. Consequently it slowly yields here to the osmotic pressure of the dissolved substances in the vacuole pressing the protoplasmic plug against it. As it yields, the growth of the cytoplasm, the subdivision of the nuclei and chloroplasts and the secretion of more cellulose, where the wall pulls out, keep pace with the elongation. So the length of the branch is increased, while the materials assimilated by the cœnocyte are being constantly used up. 
The assimilated materials are also utilised for the production of new individuals.

The reproduction of Vaucheria is characteristically effected by an asexual and a sexual method.

In the asexual method the terminal mass of protoplasm of one of the ends of the branches becomes cut off from the rest of the cœnocyte by a partition of cellulose. The chamber thus cut off is distended into an ovoid form, possibly owing to an increase in the osmotic pressure of the vacuole enclosed in the isolated mass of protoplasm. Within the inner zone of the cytoplasm of this portion the nuclei marshal themselves with great regularity so that they are equally spaced from one another and are only one deep. Outside them the chloroplasts lie in the peripheral cytoplasm. With this arrangement of its parts the protoplasm contracts a little and draws away from the wall, and forms a rounded mass lying free within the ovoid chamber. Cilia now appear on the surface of this mass, a pair radially opposite each nucleus, and by their lashing motions cause the mass to rotate in the chamber. The wall forming the distal end of the latter partially dissolves and a hole is formed, which the motile mass within is not long in finding out. Usually the hole is too small to allow this mass, which is called a zoospore, to pass through with ease, and the zoospore has to undergo considerable distortion before it escapes. Sometimes in its struggles to be free, when part of it has already emerged the remainder is detained by the constriction formed by the opening, the zoospore parts in twain and the anterior swims away leaving its less fortunate half to emerge later.

The zoospore swims about for perhaps 15 minutes. During this time it is positively phototropic, i.e. it acts just like Volvox and swims towards the light, but it is also apparently sensitive to other stimuli, for its course is seldom exactly defined by the direction of the light. At the end of this time its motions become sluggish and ultimately it comes to rest. It casts off its cilia and secretes a cell-wall. The cell-wall is at first ovoid, enclosing an egg-shaped mass of protoplasm. The latter is deep green in colour owing to the presence of large numbers of chloroplasts. If conditions are favourable the protoplasm within soon begins to grow and pushes out into a tubular plant covered with the cell-wall which keeps pace in its growth. Rhizoids and branches appear in due course and thus a new individual is established.

Sexual reproduction is also found in Vaucheria. Special branches are formed on the cœnocyte to carry out this process. 
The branches are of two kinds and are usually formed close together. The oogonia are short rounded branches the cavity of which is separated from that of the cœnocyte by a transverse wall. The branch or oogonium is egg-shaped with an obliquely placed apical prominence. The cell-wall covering this prominence becomes gelatinous and soft during the later stages of the development of the oogonium. The oogonium is filled with a mass of protoplasm-the ovum. In the centre of the ovum a nucleus may be seen. Round the latter, in the peripheral layers, are crowded great numbers of chloroplasts which make the whole oogonium dark in colour. In the immediate neighbourhood of its apical prominence or beak the chloroplasts and other granules are absent, and the protoplasm at this spot is transparent and colourless. This colourless region of the ovum is called the receptive spot. The oogonium arises as a small swelling on the side of a branch of the cœnocyte. Cytoplasm carrying nuclei and chloroplasts moves into this swelling or bulge. Subsequent growth converts this excrescence into a very short, thick cylindrical branch and a cross partition cuts it off from the rest of the cœnocyte. In some species of Vaucheria, just before this partition is completed, all the nuclei save one retire into the conocyte. In the species of Vaucheria where they remain in the oogonium, after it has been isolated by the partition, all the nuclei except one undergo a process of disintegration. In either case only one nucleus survives in the oogonium. The latter now begins to swell and takes on its mature egg-shaped form.

Simultaneously with the oogonium, the antheridium is formed and usually in close proximity. The antheridium is a slender, curved, cylindrical branch. Protoplasm from the supporting conocyte moves out into this branch as it grows. When its length is about 4-8 times its diameter and the antheridium has become much more curved, a transverse septum of cellulose is formed in it dividing off a terminal portion. This portion contains a large number of nuclei and chloroplasts. The former collect in the central region while the chloroplasts mass at the periphery. The central mass of protoplasm now breaks up into a large number of tiny masses each containing a nucleus and carrying two cilia. The cilia are placed far apart and diverge from one another. These very small motile cells are the sperms. As they develop they take on a dancing motion. The tip of the antheridium soon opens and the sperms are ejected into the surrounding water. Some of the outer protoplasm is thrown out with them and some remains in the antheridium. 
At the same time as the ejection of the sperms the solution of the wall covering the beak of the oogonium is completed and the sperms seem detained at the opening in the wall of the oogonium so formed. Often several of them enter. One only, however, fuses with the ovum, penetrating at the receptive spot. Its cytoplasm merges with the cytoplasm of the ovum. In stained specimens the passage of the nucleus of the sperm across the cytoplasm of the ovum and its ultimate fusion with the nucleus of the latter have been observed. Thus fertilisation is effected and the oosperm, or cell formed by this union of the ovum and sperm, contracts in the oogonium and secretes round itself a comparatively thick cell-wall. In this condition it undergoes a period of rest. When germination occurs the oosperm grows directly into a new plant.

These two methods of reproduction in Vaucheria are evidently suited for different conditions. Thus the zoospore germinates immediately after its cilia are cast off and its cell-wall is formed, while the oosperm remains dormant for a period. The latter is able to resist considerable desiccation, and consequently can survive unfavourable conditions. Further, it has been found that the two processes are evoked by different stimuli: zoospores are formed in response to increase of light or a scarcity of food; sexual reproduction is initiated in response to a supply of sugar, a high temperature and a bright light.

In Volvox we observed a differentiation into reproductive and somatic cells; also among the latter category, specialisation was noticeable. The somatic cells round the anterior pole are furnished with stigmata and are apparently capable of perceiving light, those round the posterior pole are not furnished with these organs. The differentiation of Vaucheria is quite as marked, but of another kind. Certain branches of the cœenocyte are furnished with chloroplasts and these function as organs of photosynthesis; others, the rhizoids, are colourless and to them the function of anchoring the cœnocyte is assigned. Thus in both cases structural differentiation and division of labour are associated.

\section{PRACTICAL WORK.}

Detach a portion of Vaucheria from the felt or mat of fibres covering moist soil, causing as little injury as possible to the filaments. Mount it in water and cover.

After examination of the plant, make drawings of three optical sections, one in a plane immediately within the cell-wall, where it is nearest the observer, one in a median plane and another in a plane in the protoplasm at 
the far side of the cœnocyte. From comparison of these three drawings reconstruct a transverse section.

Examination of material which has been submerged in shallow water for variable periods and exposed to dull or bright light will afford instances of the production of zoospores and of the sexual cells. Mount some pieces of the cœnocyte in liquor iodi. Note the absence of the starch reaction.

Make drawings of an oogonium, an antheridium and a zoospore if you can find specimens. 


\section{LECTURE XII.}

MUCOR may be found growing on various moist organic substances. A piece of bread kept moist under a bell-glass usually shows specimens of this plant in one or two days if kept at an ordinary room temperature.

The growth which is first noticed resembles very fine threads or filaments of cotton-wool lying along the surface of the moist bread and often spanning the little depressions and irregularities of its surface. If these are examined carefully by probing with a pin or a needle they will be found to branch, and it will be discovered that they are held down by some of the branches which penetrate into the bread. By continued development and branching of the filaments the growth assumes the appearance of a felt covering the bread more or less closely. The filaments forming this felt and the branches which penetrate into the bread are called the mycelium. After a day or so the mycelium sends up vertical branches varying from $2 \mathrm{~mm}$. to $2 \mathrm{~cm}$. in length. At first these branches which are quite straight and cylindrical have a rounded end and like the horizontal branches of the mycelium are pure white. Shortly, however, small globules begin to develop at their tips and these darken in colour until they are quite black. When they have developed this black colour the slightest touch suffices to make them burst and to discharge a whitish mass of mucilage. In addition to these globules which range from translucent white to black, very often limpid drops of fluid may be seen adhering to the vertical branches.

Microscopic examination of the mycelium and of its vertical branches shows the following: the mycelium consists of a single cœnocyte, on rare occasions showing transverse septa. The appearance of the creeping branches and those which penetrate the bread are quite similar, both being covered by a delicate cellwall within which is a film of protoplasm. They are called hyphæ. A clear colourless vacuole occupies the axis of the branch. There is no appearance of chloroplasts or of any other coloured bodies in the protoplasm or elsewhere. If one of these 
hyphæ is killed and stained, the cytoplasm may be very plainly observed lying in contact with the inside of the cell-wall and forming a film of irregular thickness coating its inner side. Sometimes one sees filaments and bridges of cytoplasm traversing the axial vacuole. In the cytoplasm scattered nuclei may be seen. Treatment with iodine brings no starch-grains to light but in their place scattered oil-drops or fat-globules are found in the cytoplasm. The hyphæ of the mycelium taper gradually towards their tips. The latter appear filled with protoplasm. Observation shows that the growth of the mycelium is localised at these tips. The vertical branches have the same structure as the other hyphæ
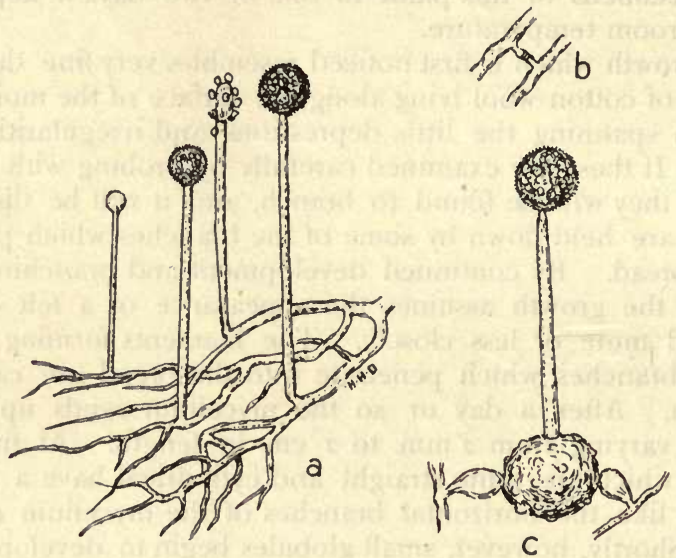

FIG. I7.-Mucor. $a$, mycelium with sporangia in different stages of development, $\times 70 ; b$, conjugation-branches in contact with one another; $c$, zygospore supporting a sporangium, $\times \mathrm{I}_{50}$.

and are covered with the same continuous cell-wall. The protoplasm and vacuole are usually continuous throughout the whole plant.

Although Mucor is not motile like Volvox, the direction of its growth has been shown, like the motion of Volvox, to be determined by external stimuli. Thus it has been found that the growing tips of its hyphæ are oriented in such a manner that they grow towards the point of greatest concentration of substances useful in their nutrition. It may also be inferred from analogy with other plants, that harmful substances:-or harmful concentrations exercise a repellent action on these hyphæ. 
This directive action of chemical stimuli is termed chemotropism or chemotaxis. The different behaviour of the vertical, creeping and penetrating branches must, in the first instance, be due to their difference in response to similar stimuli. Consequently, although we can discover no microscopic difference in the protoplasm of these organs, there must be some physiological difference which is responsible for their different behaviour. The emergence of the vertical branches appears to be due to negative hydrotropism, i.e. a response which causes them to grow from a region where the concentration of water vapour is great towards one in which the concentration is less. Once the vertical branch has emerged the most important directing stimuli appear to be gravity and light. Under usual conditions both stimuli act, and consequently the branch takes up an intermediate position due to two responses. If the branch is removed from the action of one, say of light, and is grown in the dark, its response to gravity may be studied alone. It will then be found that the vertical branches are negatively geotropic, i.e. tend to curve until their growing tip is directed away from the centre of gravity of the earth. This causes these branches under normal conditions to assume and maintain a vertical position. The branch will curve in this way as often as it is disturbed from the vertical position. If the piece of bread from which several vertical branches are emerging be set on its side, so that the surface which was before horizontal becomes vertical, under the new conditions the emerging branches will slowly bend through $90^{\circ}$ and bring their tips once more into a vertical position.

The mechanism of this movement is quite obscure. The rigidity of the branch in the vertical position is due to the osmotic pressure of the solutes in the vacuole pressing out the lining of cytoplasm and putting the cell-wall in tension. The solutes represent the compression member and the cell-wall the tension member in this rigid structure. If the member in compression be removed-as we may remove it by rendering the cytoplasm permeable by heat - the branch will collapse. This internal pressure is apparently also an important factor in the growth of the branch ; it pushes the terminal part forward where the cell-wall is most ductile. Hence we may assume that the unequal growth of curvature is produced by one side of the wall of the growing region being more ductile than the other and yielding more readily to the internal pressure. This softening of the cell-wall may very probably be caused by the action of an enzyme made more effective on one side by the accumulation of some substance acting as a coenzyme. The accumulation of hydrogen ions has been suggested. 
Just as the stimulus of gravity tends to keep the branch growing in a vertical line, so the stimulus of light tends to orient it in the direction of the incoming light. But these branches are positively phototropic, that is they tend to direct themselves towards the source of light. No more is known regarding the mechanism of the reaction to the phototropic stimulus than is known regarding that of geotropism.

The top of the vertical branches is at first rounded off. As the branch increases in length its end swells into a tiny knob. This is at first white but as it grows, it becomes greyish and darkens till it finally appears as a black speck or globule on the top of the branch. Microscopic observations on the development of this globule show that in the early stages, when it begins to swell, a transverse septum is formed at the end of the vertical branch cutting off the enlarging tip from the rest of the cœnocyte. The protoplasm within the tip has many nuclei and they further increase in number by subdivision. At the same time the wall covering the globule grows thick, it becomes pigmented and a fine deposit of crystals of calcium oxalate is formed on its outer surface. The protoplasm within this capsule begins now to cleave into fragments. At first each of these contains several nuclei but cleavage continues until the number of fragments is equal to that of the nuclei, and each thus becomes uninucleated. The fragments contract and become ovoid, each secreting a cell-wall round itself. They are immersed in a watery mucilage which fills the capsule. Just when the fragments or spores, as we may now call them, have assumed a cell-wall, the transverse septum, separating the capsule or sporangium from the vertical branch, bulges from below upwards into the sporangium thus allowing the upper part of the supporting branch to invade the sporangium. The supporting branch is called the sporangiophore, the intruding part is called the columella. The intrusion of the columella naturally distends the sporangium and often bursts it, or renders it so tense that it explodes at the slightest disturbance, setting free its spores in the drop of contained mucilage. If these spores find their way to a suitable organic medium they will soon germinate by pushing out hyphæ which branch and quickly form a mycelium. Later the vertical branches or sporangiophores appear and produce sporangia which liberate spores once more. So the cycle repeats itself.

Sexual reproduction also occurs in Mucor, but much less often than the asexual production of spores just described. When the hyphæ of two mycelia growing in the same medium come into 
close proximity to each other, short club-shaped branches arise sometimes on the adjacent hyphæ and grow towards each other. When their ends come into contact a transverse septum develops in each, cutting off the protoplasm of the slightly dilated end from the rest of the branch. The walls at the point of contact become dissolved and the protoplasm of the two ends mingles. By suitable stains it has been shown that there are many nuclei in the ends of the two branches before they unite; these nuclei fuse in pairs from the opposite branches. The composite mass of protoplasm thus produced becomes coated over with a thick cell-wall, the outer layers of which become dark and warty. The whole structure is called a zygospore or zygote. Its thick wall enables it to resist desiccation. After some time of dormancy its coat breaks and a vertical branch emerges from it. On the apex of this a sporangium is produced just like that formed on the sporangiophores of the mycelium. The spores liberated from the sporangium give rise to new mycelia.

In this. sexual reproduction the two branches resemble each other completely and the sexual organs are cœnocytic, and just as the cœnocytic zoospores of Vaucheria appear to represent a mass of undifferentiated, unicellular zoospores, so the contents of these branches should be regarded as a number of undifferentiated, unicellular gametes.

While it is impossible to detect any difference between the two fusing branches or the mycelia from which they come, it has been found that fusion or sexual union will only take place when the mycelia growing together have distinct origins. Thus the mycelia developed from spores originating from the same sporangium will not unite, but fusion may occur between two distinct strains cultivated together. Evidently some invisible sexual differentiation is concerned. We must imagine some substance, or form of energy, emitted by the hyphæ which acting differentially on the two kinds of mycelia, evokes the production of gametes from one; while the other under the same stimulus continues to produce vegetative branches.

Mucor grows well when supplied with proteids and carbohydrates. It is also able to nourish itself as a parasite. As a general rule, however, it draws its nutrition from dead organic matter and is to be classed among the saprophytes. Not having any chlorophyll or other photosynthesising pigment, it is absolutely necessary for it to find organic substances containing carbon. It is also unable, like other plants not possessing chlorophyll, to build up proteins from inorganic substances and it requires 
supplies of organic nitrogenous substances. It cannot make use of nitrates for this purpose.

Unlike yeast it normally requires free oxygen for its respiration. It is, however, capable of life in a submerged condition. The supply of free oxygen is then limited and in response to this changed condition the hyphæ undergo transverse division. The portions of the hyphæ so formed each gives rise to a rounded cell not unlike that of yeast. These cells when introduced into a sugar solution bud and behave very like yeast-cells. They change the sugar of the solution into alcohol and carbon dioxide and thus show that, in this form at least, Mucor is able to subsist by means of anaerobic respiration.

\section{PRACTICAL WORK.}

Examine a growth of Mucor on moist bread with a lens. Make out the creeping hyphæ and the vertical branches with mature and immature sporangia. Cultures for microscopic examination may be made of the "spontaneous growth" on bread as follows: Draw a sterilised needle through the vertical branches, not letting it touch the surface of the bread, and with it innoculate a test-tube containing bouillon gelatine. Stir the liquid gelatine well with the needle to distribute the spores. With a sterile glass-rod put drops of the gelatine while still liquid on to sterile micro-slides which should be set aside and kept moist under a bell-glass. The slides may be taken out at intervals and examined microscopically. If it is intended to use a high objective in the examination the preparation should be covered. Before covering a drop of alcohol is added to prevent the inclusion of air bubbles. The cover-glass is then laid on the preparation, and the slide is gently warmed over a flame so that the cover-glass settles into position by the melting of the gelatine.

In these preparations the early stages of germination should be studied and the growth and structure of the mycelium. Beautiful preparations may be made by first fixing the growing mycelium with iodine vapour and then staining with Delafield's hæmatoxylin. This may be done by inverting the slide and laying it across the neck of a bottle containing iodine crystals, so that the preparation hangs into the neck of the bottle. Fixing is accomplished in a few minutes and then a drop of Delafield's hæmatoxylin is added to the gelatine and the slide returned to the moist chamber for 30 minutes or an hour. At the end of this time the stain should be washed away, the preparation covered and warmed gently. This preparation will keep for several days and shows the cytoplasm a pale violet while the nuclei appear as dark purple globules.

The vertical branches should be examined. In covered preparations they will of course be pressed down on the gelatine. The sporangia will be seen in different stages of development with the transverse septum at first flat and later bulged into the sporangium and forming the columella. The differentiation of the spores may be traced and the changes which convert the wall of the sporangium from a smooth, colourless membrane to a thick greyish wall studded closely with tiny crystals.

The diameter of the thickest hyphie and the size of the spores and sporangia should be estimated by means of the Ghost-micrometer.

Spores sown in gelatine bouillon produce plants which bear ripe sporangia in two days if the cultures are kept at ordinary room temperature. 


\section{LECTURE XIII.}

Plants which, like Mucor, do not possess chlorophyll and are not differentiated into leaves, stems and roots like the higher plants, are called Fungi. Their want of chlorophyll renders it impossible for them to form organic substances from inorganic, and hence they are compelled to derive their food from pre-existing organic substances. The fungi are therefore parasites or saprophytes. Of the plants which we have already studied Yeast and Mucor are fungi. Another very common fungus is Penicillium glaucumcommonly called blue mould. Like Mucor it is found growing on various moist organic substances, but it is even more common than Mucor.

The mycelium of Penicillium resembles that of Mucor in general appearance. It forms a white felt of delicate filaments or hyphæ spreading over the surface of the substance on which it is growing. Microscopic examination shows, however, that its hyphæ are subdivided into a number of segments by transverse walls. These segments are cylindrical, and a fairly thick layer of highly refringent protoplasm may be seen lying against the inner surface of their cell-walls. The axial vacuole is broken up into a number of spherical droplets by thick diaphragms of protoplasm extending across the cavity of the cylinder. The protoplasm appears homogeneous and is comparatively free from granules. Some of the few granules which do appear may be shown by staining to be nuclei, others are minute oil drops. The number of nuclei in each segment is small, perhaps 4-8. Hence the segments are small coenocytes. Another point of difference wherein' the hyphæ of Penicillium are unlike those of Mucor is the fact that the hyphæ of the former do not taper, but are uniform in thickness right up to the growing tip. The terminal cœnocyte of a hypha has a rounded end and this is filled with protoplasm so that there is no room for a vacuole. Elongation of this cœnocyte takes place just like the elongation of the whole cœnocyte of Mucor, but as soon as it has grown in length so as to equal once and a half or twice the length of the mature cœnocytes of the hyphæ a transverse 


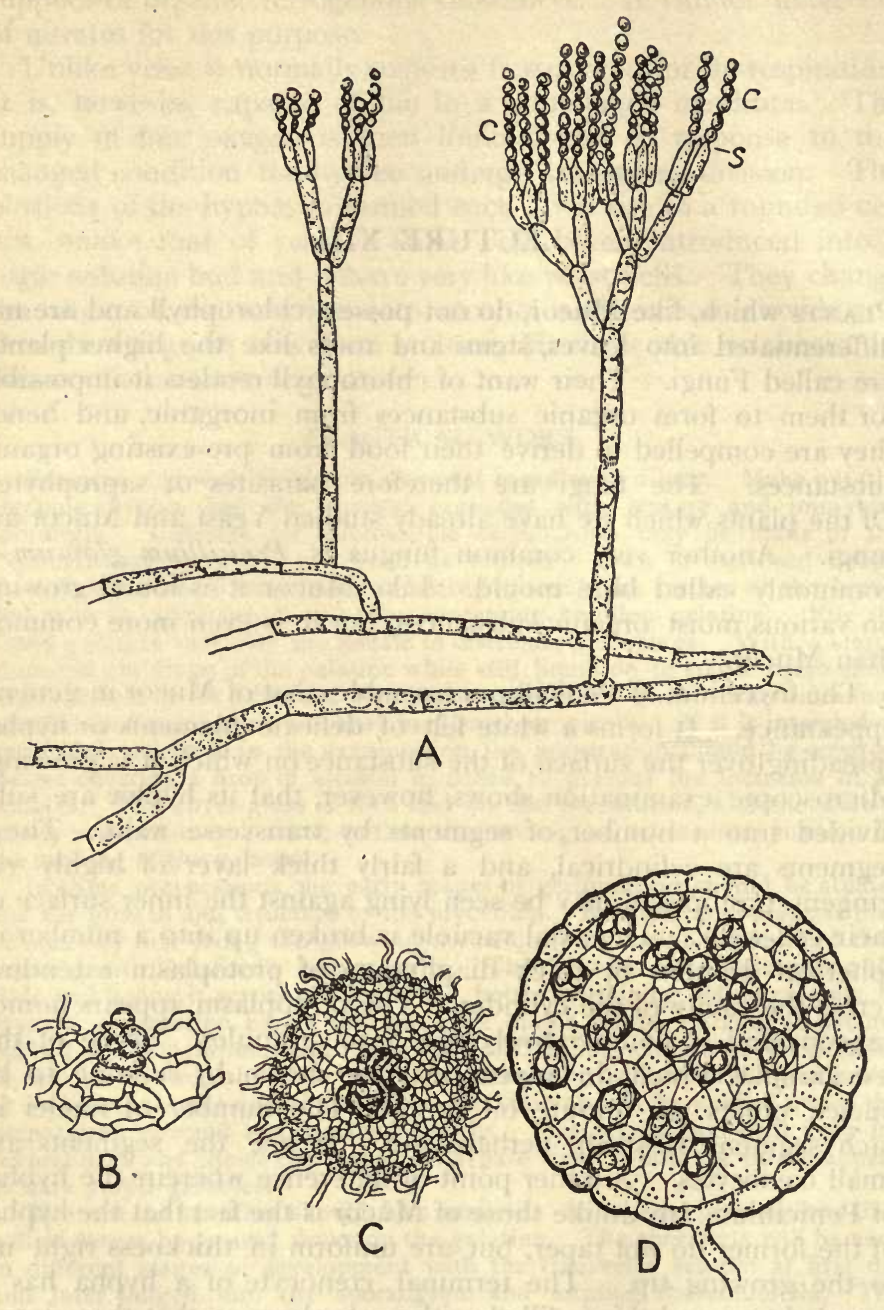

FIG. I8.-Penicillium glaucum, $\times 4$ 0o. A. part of mycelium with condiophores; $c$, c inidia; $s$, sterigma. B, C and D, stages in the formation of the ascocarp. (After Brefeld.) 
wall is formed cutting off the posterior more vacuolate portion as a separate segment from the growing tip, which continues its forward growth. Once the segments are thus cut off from the growing apex they cease to elongate markedly. Lateral branches develop as outgrowths from the segments usually immediately behind the transverse wall nearest the apex. These outgrowths push out to form new cylindrical growing segments and by elongation and subdivision develop into new lateral hyphæ. By a continuance of this process from many segments of the first hypha complicated branching systems are developed. As the growing tips of all the hyphæ respond chemotropically in a positive sense to the concentration of food substances in the medium, the whole mycelium spreads in a radial manner, and extends from the region where the food-substances are exhausted by its own absorption to the surrounding regions as yet untapped by its inroads.

When growth of the mycelium has proceeded for four or five days upright branches are formed, the first appearing in the oldest and consequently the central parts of the mycelium. They resemble the hyphæ of the mycelium, so far as can be seen, except in their response to external stimuli, which causes them to assume a vertical instead of a horizontal position. Each vertical branch consists of an unbranched shaft formed of four or five cylindrical segments. The rigidity necessary to retain them in the vertical position is conferred on these branches by the osmotic pressure within the vacuoles of the cœnocytes, as may be seen when the cytoplasm is rendered permeable by iodine vapour or bisulphide of carbon. On the top of these are supported segments which give rise to lateral branches, and the segments of these latter may give rise to more branches. As all the branches formed respond in the same manner towards gravitational force they arrange themselves parallel to one another and vertical to the mycelium. The vertical branching system reminds one of a complicated candelabrum. On the terminal conocytes or segments of this branch-system, which is called a conidiophore, a small group of peculiar shaped cœnocytes is formed. Each of these is bottle-shaped, having a cylindrical basal portion continued into a narrow neck-like part. The latter is called the sterigma. The bottle-shaped cœnocytes occupy the position of the candles in the candelabrum. The tip of each sterigma is covered with a plastic wall and the internal pressure in the coenocyte bulges it out to form a minute globule - a conidium. This is a tiny mass of protoplasm containing a nucleus, and soon its covering membrane becomes 
thickened and loses its extensibility. While it is thus being matured a second conidium is produced from the sterigma below and pushes up the first. Repetition of this process produces a chain of conidia from the tip of each sterigma. The older conidia forming the outer end of the chain have a blue coloration in their walls which confers the characteristic blue colour on the tips of the mature conidiophores and has caused this plant to be called blue mould in contradistinction to Mucor, which not having this colour, is called white mould. These older conidia are very easily detached from the sterigmata and being very minute $(2 \mu$ in diameter) are readily borne on the feeblest draughts of air and scattered far and wide. When they fall on suitable substances they absorb water and their protoplasm, which at first filled the cell-wall entirely, now becomes vacuolated. The wall is thus distended and the conidium increases in size. Then it forms a bulge and this grows out into a cylindrical cell. The nucleus subdivides and so the cell becomes a cœnocyte, which elongates, divides and so gives rise to a hypha. By branching in the manner described above, the hyphæ develop into a mycelium and the latter ultimately produces conidiophores and conidia, thus completing the life-history. The conidia are single asexual cells and may be classed as spores according to our definition.

Reproduction by means of conidia occurs in the normal growth of this fungus and is very prolific, but when it develops under conditions in which the supply of oxygen is limited, Penicillium resorts to another method-the sexual one.

This process is begun by the development on the mycelium at numerous places of pairs of short branches, which we may provisionally call gametangia. These gametangia are cylindrical, their bases are close together and they coil round one another spirally. Actual fusion of the protoplasm of the two gametangia is believed to take place, but has not been observed. The coiled gametangia now give rise to short branches called ascogenous hyphæ which grow out in every direction. Meanwhile the hyphæ which support the gametangia also form numerous branches which grow up over the ascogenous hyphæ interlacing in a confused tangle with one another and forming a compact covering over them of several layers in thickness. The walls of the outer layers become thickened and dark-yellow in colour and form a hard, resistant rind to the mass which is now spherical in form. After a pause in growth of several weeks the ascogenous hyphæ form a series of peculiar thick branches with hook-like 
bends, which give rise to chains of ovoid cells-the asci. In each ascus eight ovoid cells, ascospores, are found. In addition to the hooked branches the ascogenous hyphæ produce narrow tubular branches which penetrate into the inner layers of the envelope. These tubular branches seem to have the function of absorbing the materials-proteins and cellulose-stored in the inner layers and of transferring them to the developing ascospores. Not only are these cells absorbed, but even the ascogenous hyphæ and the walls of the asci are dissolved, so that finally only the outer brownish-yellow wall of the envelope containing the ascospores is left. The envelope of this fructification or ascocarp cracks open when it dries and the ripe ascospores are set free. The formation of the ascocarp from the production of the gametangia to the liberation of the ascospores takes from six to eight months.

The ascospores have a diameter about twice that of the conidia. They germinate on moist organic surfaces by cracking open the outer yellow layer of their cell-wall and pushing forth an outgrowth which develops into a mycelium.

Penicillium may be found growing on a great variety of organic substances and hence is a very common plant. This shows that it can supply its needs from a large number of different materials. It is in fact omnivorous. Apparently this adaptability is closely connected with its great wealth in enzymes. Probably from no other plant have so many enzymes been isolated as from the subject of our study. Penicillium has been shown to use diastase for liquefying starch and converting it into maltose, cytase for rendering cellulose soluble, invertase, inulinase and zymase for breaking down other carbohydrates ; it has emulsin for glucosides, protease and casease for attack on proteins, lipase for fats and catalase which sets oxygen free from peroxides. It is probable that a further search would bring to light a still larger variety of enzymes in the répertoire of this extraordinary plant.

By these enzymes it is enabled to draw the carbon it requires for its growth and respiration from proteins, carbohydrates, fats, organic acids and alcohols.

Notwithstanding its omnivorous character Penicillium preserves a refinement of discrimination which would do credit to the most experienced epicure. This discrimination is strikingly shown in its attitude towards tartaric acid.

Pasteur found that when Penicillium grew in a nutritive solution containing tartaric acid, the acid which before was optically inactive became optically active. That is to say, it acquired the power of rotating the plane of polarisation of a beam of polarised 
light passing through it. This surprising observation led Pasteur to the discovery that the tartaric acid which he supplied to the plant was a mixture of two substances resembling each other chemically, both having the empirical formula $(\mathrm{HCOH})_{2}(\mathrm{COOH})_{2}$, but differing from each other in the physical characteristic that one rotates the plane of polarisation clockwise and the other counter-clockwise. In the mixture these two effects counterbalance each other and the polarised beam is not rotated. But when Penicillium is in contact with the mixture it assimilates the tartaric acid which rotates the plane to the right ( $d$-tartaric acid) while it practically leaves untouched that which rotates the plane of polarisation in the opposite direction ( $l$-tartaric acid). Hence, it may be assumed that Penicillium possesses an enzyme which can render $d$-tartaric acid suitable for its metabolism while this enzyme is ineffective with $l$-tartaric acid. The discovery of the two forms of tartaric acid revealed by the selective action of Penicillium led to the further discovery of a large number of other carbon compounds in the molecules of which the atoms and atomic groups are capable of being arranged in several different ways round certain of the carbon atoms. Distinctive physical properties depend on these arrangements while the individual atoms and atomic groups of these substances, which are called stereo-isomers, remain the same. Thus it becomes very probable that the means by which Penicillium can deal with dextro-tartaric acid is ineffective with lævo-tartaric acid because it fits the atomic arrangement of the one and does not fit that of the other. This case and others of a similar nature have led to the "Lock and Key" theory of enzyme action. According to it the specific action of an enzyme on a certain substance depends on the fact that the spatial arrangement of the atoms and atomic groups in the enzyme-molecule fit in some way the arrangements of the atoms in the substance acted upon.

Comparatively simple nitrogen compounds form a suitable supply of nitrogen for Penicillium. Thus it can utilise amidocompounds and peptones.

We have seen that fungi resemble animals in their nutrition. They are both dependent on organic carbon compounds to supply them with the carbon they require, and they are as a rule unable to utilise inorganic nitrogen as a supply of that element. Hence, they are classed together as holozoic organisms. Many fungi, however, can utilise much simpler nitrogenous compounds than animals. Thus yeast can utilise ammonium tartrate as a source of nitrogen, while it is found that Penieillium thrives on ammonium 
nitrate. On the other hand, the higher animals at least require a supply of the more complicated nitrogenous compounds such as some of the higher proteins. This would indicate that plants alone can build up the nuclei of these substances and that the rest of living nature is in this matter, as well as in carbohydrate formation, dependent on them.

Under normal conditions Penicillium respires free oxygen and of course gives off carbon dioxide and water. Its respiration is aerobic. It is found that the ratio of the volume of carbon dioxide given off to the volume of oxygen absorbed (respiratory ratio, $\mathrm{CO}_{2}: \mathrm{O}$ ) is dependent on the source of carbon supplied to the mould; thus with sugar the ratio is $\mathrm{I}$, with tartaric acid it is 2.9 .

When Penicillium is cut off from a supply of free oxygen it is found to be able to subsist by anaerobic respiration. As with yeast, during this process carbon dioxide is evolved and alcohol is produced. It has already been pointed out that anaerobic respiration is incomplete combustion. Consequently it does not render available to the plant the maximum amount of energy contained in the carbohydrates at its disposal. When the conidia germinate under conditions where anaerobic respiration only is possible they bud in a manner closely resembling yeast cells.

\section{PRACTICAL WORK.}

From a growth of Penicillium on moist bread isolate a portion with a needle, mount it in a drop of spirit, add a drop of water and cover. It is necessary first to moisten with spirit because water alone will not wet the conidıa, which behave as if they were greasy.

Put drops of peptone-broth-gelatine on three or four sterilised microscopic slides. When it is set stab each with a sterilised needle which has touched the conidia of a pure culture derived from the growth on the bread. Put the slides aside in petri-dishes moistened on the inside and examine on successive days. For examination it is well first to moisten with a drop of spirit, then with water and cover. The preparation should then be gently warmed to allow the cover-glass to settle into position.

Careful drawings should be made of the germinating conidia and of the hyphæ to which they give rise, showing the cœnocytic segments and the mode of branching.

When the conidiophores are mature a preparation should be exposed to iodine vapour. Note the collapse of the stalk when the protoplasm of the cœnocytes has thus been made permeable. Stain the specimen in dilute Delafield's hæmatoxylin for 24 hours and mount and examine. Careful draw ngs should be made and estimates of size recorded on the drawings by means of the Ghost-micrometer. 


\section{LECTURE XIV.}

Fucus or sea-wrack is one of the best-known sea-weeds. It contributes more than perhaps any other sea-weed to cover the halftide rocks of our coasts. It has an olive-brown colour. When examined it is seen to be composed of a small disc-shaped sucker, by means of which it is attached to the rock, and a cord-like stem which supports a flat branching portion. The bifurcations of this portion are in one plane and the branches are flattened in the same plane. The stem appears to be continued up into the flattened portion or frond as a cord-like mid-rib which may be traced upwards almost to the extreme tips. The mid-rib bifurcates with the branching of the fronds. The surface of the plant is slippery and mucilaginous to the touch and the edge of the frond is more or less notched. Some kinds are possessed of bladders filled with gas formed in the tissue of the fronds at irregular intervals along the mid-rib; and often the ends of the fronds are swollen into cushion-like enlargements. The general surface of the plant is smooth but it is studded over with what appear to be small warts when the plant is out of water. These structures are most noticeable and most numerous on the swollen ends of the branches. When the plant is submerged it is easily seen that the wart-like bodies are tufts of fine hairs which issue from the top of a slight prominence.

If the frond of Fucus is cut across it will be seen that it is composed of two distinct layers. The outer or cortical layer, which is comparatively tough and is coloured brown, and an inner medullary substance which is transparent and almost colourless and has a mucilaginous consistency.

If a thin transverse section of the frond be prepared and examined microscopically we shall see that the cortex is composed of several layers of cells coloured a golden olive-brown. The outer ones, which form a continuous coating covering over the whole frond, appear square in outline in the transverse section. Their walls are thin except those which form the outer surface of the plant, and these outer walls together form a sheet which may 
be called the cuticle. Within the superficial layer the cells of the cortex are larger and they increase in size from without inwards. They are polyhedral and they measure approximately the same in every direction. (Cells with this shape are called isodiametrical.) Like the superficial cells they have thin walls and they appear completely filled with protoplasm, in which is embedded a single nucleus and countless very minute chromatophores. These latter contain the olive-brown colouring matter which gives the colour to the frond. Like the chloroplasts of the green cells we have examined, the chromatophores are made of protoplasm and they contain the four pigments found in the chloroplasts, viz. chlorophyll $a$ and $b$, xanthophyll, and carotin. In addition to these four there is in them another brownish pigment, fucoxanthin. While in the green plants the quantity of the two chlorophylls present in the chloroplasts is about five or six times that of the yellow pigments, in the chromatophores of Fucus the ratio of the yellow plus fucoxanthin to the chlorophylls is $12 / 17$. This difference accounts for the difference in colour. The presence of the chlorophyll may be very easily demonstrated by immersing the frond in hot water. This causes the yellow pigments to bleach and the undiluted green of the chlorophyll present becomes apparent. The same result may be obtained by a short immersion in spirit.

As might be expected, the chlorophyll in these cells is present to enable the plant to form carbohydrates from the carbon dioxide in the surrounding water. When Fucus has been exposed to bright light a granular substance is found in these cells. It is probably a carbohydrate. Thus we realise that Fucus derives its carbon by photosynthesis in a manner similar to other plants possessing chlorophyll.

The inner layers of the cortex are composed of cells with more irregular shapes than those of the outer layers just described. They are elongated in one direction and have thicker cell-walls. Their protoplasm does not completely fill the cavity of the cell and a vacuole of considerable dimensions is seen. The chromatophores are not so densely crowded in the protoplasm. The change from the outer to the inner cortex is not abrupt but the characters of the cells of the two layers grade into one another. The central region of the frond is called the medulla. It is composed of strands of elongated cells which resemble hyphæ, and cohere loosely together at irregularly spaced points of contact, leaving irregular spaces between them. Branches from the cells of the inner cortex penetrate between the hyphæ of the 
medulla. Like the cell-walls of the inner cortex, those of the medullary cells are thick. Where the cells come into contact with one another the walls have thin spots (pits) upon them and these thin spots are finely perforated. Through these perforations excessively fine strands of protoplasm pass, linking up the protoplasm of adjacent cells. Similar connections are found between the cells of the cortex. The cells of the medulla possess very few chromatophores but they are peculiarly rich in tannin granules. Evidently the tissue of the medulla acts as a storehouse for the latter material.

In the mid-rib the medullary hyphæ are disposed parallel to one another and run longitudinally in the frond. Their elongated form, added to the fact that they possess terminal perforations in their walls, fits them probably for transmitting substances and for conveying stimuli in a longitudinal direction.

Where the transverse section passes through one of the tufts of hairs emerging from the surface, it will be seen that the hairs take their origin from the sides of a small depression in the surface of the frond and pass out through a small orifice. The margin of the orifice is often slightly raised above the surrounding tissue. The hairs expose a large surface to the surrounding water and probably function as absorbing organs for the mineral salts needed by Fucus in its nutrition.

Observation shows that Fucus grows by additions made to the ends of the branches. On the very edge or tip of a growing branch there is a small groove running in the same plane as the frond is extended. Microscopic investigation shows that the bottom of this groove is formed of a linear series of large rectangular cells which frequently divide by means of partition walls. These walls cut off segments parallel to the inner and longitudinal sides or faces of the cells. The segments formed on the longitudinal faces subdivide and give rise to the cells of the cortex while those cut off from the inner surface develop into the medullary hyphæ. Thus from the growing groove all the tissues of the plant are formed.

Sometimes the middle cells of the series forming the bottom of the groove cease dividing while those towards either end continue. In this way two groups are formed from the originally continuous series and as each continues growth and subdivision two growing regions are developed, each of which ultimately gives rise to a branch, and bifurcation, or dichotomy, results.

Normally the central cells of the groove give rise to the mid-rib composed of its elongated medullary hyphæ and the cortex, while 
the ends of the groove form the flattened parts of the frond on each side of the mid-rib. These latter persist only for a time and as the apex grows and forms new tissues the older tissues at each side of the mid-rib gradually wear away and leave the mid-rib alone persisting. The mid-rib thus stripped of the lateral parts of the frond persists and forms the cord-like stem of the plant.

The localisation of the growth of the plant at definite spots, e.g. the tips of the fronds, necessitates a constant supply of nutritive materials to these places for the manufacture of these new cells. The supply is conveyed by the elongated cells of the mid-rib from the cells of the cortex all over the plant.

We have seen that the cortical cells manufacture carbon compounds by photosynthesis ; they also-especially the tufted hairsmay be supposed to absorb nitrates and other salts from the seawater. These materials, either in the crude form or more probably after being synthesised into proteins, are transmitted from the outer cortex through the elongated cells of the inner cortex into the medullary hyphæ and so into the mid-ribs where they are conveyed in the parallel hyphæ to the growing points.

In Fucus then we have a marked division of labour among the tissues, some carrying on absorption, some photosynthesis, some acting as channels of communication. In addition to these we have special groups of cells set aside for growth, a special part again acting as an organ of attachment, and, as we shall see, all these somatic cells are different from the reproductive cells. Such physiological specialisation, as is invariably the rule, is associated with structural differentiation. Evidently such structural differentiation is impossible in unicellular organisms in which the single cell has to discharge a number of functions simultaneously. So that it is only in multicellular organisms we can expect to find marked division of labour and with it differences in form and structure (morphological and structural differentiation) among the individual cells.

The reproductive cells of Fucus are very markedly differentiated from the somatic. They are formed in the cushion-like terminations (receptacles) of some of the branches. The receptacles are studded over with a number of wart-like prominences from which, as we have seen, delicate hairs emerge. The opening through which the hairs emerge leads into a small spherical cavity, called a conceptacle, formed in the cortex of the frond. The cells composing the wall of this conceptacle support the hairs just mentioned; among the bases of the hairs the special capsules for 
the gametes are found. They are of two kinds-oogonia and antheridia.

The beginnings of these two organs resemble those of the hairs. A cell from the cortex forming part of the wall grows out into the conceptacle ; as it elongates it divides transversely. The terminal segment projecting into the cavity becomes an oogonium or an antheridium as the case may be. The basal segment forms its stalk. In the case of the oogonium the terminal cell enlarges

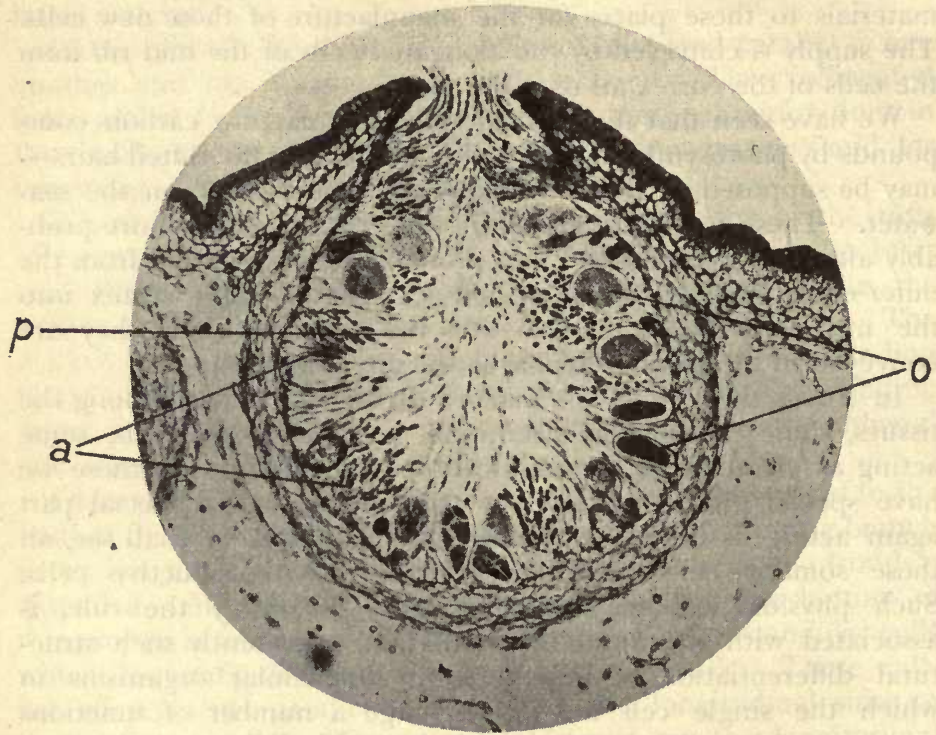

FtG. I9.-Fucus platycarpus, receptacle, transverse section passing through a conceptacle, microphotograph, $\times$ roo. $a$, antheridia; 0 , oogonia; $p$, paraphyses (hairs).

greatly and becomes ovoid so that it is just visible to the unaided eye. It is filled with dense protoplasm and is olive-brown owing to the large number of chromatophores it contains. Its nucleus divides and the resultant nuclei undergo two successive divisions, so that there come to be eight nuclei in the protoplasm of the oogonium. Round this mass two separate membranes are formed within the original cell-wall of the oogonium, and the mass divides into eight pieces each containing one nucleus. By the successive liquefaction and rupture of the original cell-wall and of the outer 
of the two inner membranes the eight masses-the ova-are cast out of the oogonium into the cavity of the conceptacle, still loosely held together by the innermost membrane.

Meanwhile the development of the antheridia is proceeding. As in the case of the oogonium, the terminal cell becomes the gametangium or sac containing the gametes. In this case, however, its protoplasm breaks up into sixty-four nucleated particles, each of which is very minute and pear shaped, furnished with two cilia, an eye-spot and a yellow chromatophore. These are the sperms. They are launched into the conceptacle, still enclosed in an inner membrane, by the rupture of the antheridium. While the development of the sperms has been proceeding the stalk cell of the antheridial hair produces another cell which displaces the first antheridium and continues the growth of the hair; branches are formed and numerous antheridia similar to the first are produced as terminal cells. We find therefore that each antheridial hair gives rise to a large number of antheridia in a prolonged succession. The branched hair with its numerous antheridia looks like a minute bush.

When the tide leaves a plant of Fucus the water imbibed in the frond evaporates slowly and causes a contraction. This seems to squeeze out the detached ova and sperms still enclosed in the inner membranes of the gametangia along with a drop of watery mucilage from the conceptacles. The drop is retained at the opening of the conceptacle by the bunch of hairs issuing through it. Here the inner membranes of the gametangia dissolve or rupture and the gametes are at last entirely free.

The very minute sperms appear actively motile and swarm round the large ova. One of them embeds itself in the surface of the latter and is gradually absorbed. After it has been thus engulfed its nucleus has been seen to pass across the cytoplasm of the ovum and make its way to the centrally placed nucleus of the latter. A fusion of the two nuclei then takes place. When fertilisation has thus been effected, the ovum loses its attraction for other sperms, and those which previously were aggregated round it move off to unfertilised ova. The oosperm formed in fertilisation immediately secrets a cell-wall over its surface. It is washed away from the conceptacle-hairs by the returning tide, settles on some solid object and immediately grows into a new Fucus plant.

No asexual reproductive cells have been found in Fucus. The great morphological and structural differentiation of the somatic cells of Fucus has its counterpart in the complete differentiation 
of its sexual cells. And in the lower forms of vegetable life, such as we have been considering hitherto, it is usual to find that differentiation of the somatic cells is associated with differentiation of the reproductive also. Hence we are led to suppose that the sexual cells of the simplest and therefore the most primitive organisms were similar to one another. Thus while the fusing gametes of Chlamydomonas are identical with one another, a physiological distinction is already visible in such a simple organism as Spirogyra, and in the more complex organisms, Vaucheria and Volvox, where already some somatic differentiation is perceptible, marked morphological and structural differentiation is apparent in the gametes. They are plainly distinguishable as the quiescent large ovum and the motile minute sperm. This differentiation is still more marked in Fucus.

Following a somewhat similar line of thought and finding that sexual reprcduction normally occurs periodically in the life history of the more complex forms, while in the simpler or lower forms reproduction is either always, or more frequently, effected by asexual cells (spores), we surmise that asexual. reproduction is the more primitive method. Asexual reproduction may be effected by simple cell division or budding (Spirogyra, bacteria, yeast), or by segmentation of the protoplasm within the cell-wall into two or more masses (bacteria, yeast, Chlamydomonas). The spores when liberated are smaller than the adult cell and evidently, unless they find adequate supplies to enable them to meet the necessary demands of energy and material, they will perish. Thus we often find spores specially provided with a store of food material such as fat, carbohydrates and proteins. Should they exhaust or lack this they will be exposed to the hunger stimulus. We may imagine that those which are naked, or only incompletely covered with a cell-wall, will, in response to this stimulus, respond as the simplest organisms do, by ingesting or engulfing other cells. The spores being set free usually in numbers together will naturally under starvation conditions tend to ingest one another and thus obtain sufficient nutriment to persist and carry on till change of conditions provides for their development. This procedure, which in the first instance was supposedly initiated by chance, might become normal by the recurrence of the same conditions. In some process like this we may present to ourselves the origin of sexual reproduction. Support of this view is found in the observation that the sexual cells of many of the lower plants may be made to act as spores (i.e. reproduce the plant without fertilisation) if they are richly supplied with food. The germination of 
a gamete without fertilisation is technically known as parthenogenesis.

In some species of Fucus the antheridia and the oogonia develop in the same conceptacle, in others they are formed in the conceptacles of different plants.

These unisexual (dioecious) individuals lend themselves to interesting experiments. Supposing the ova of one of these dioecious species be collected in a watch-glass in salt water and into the same glass the sperms of a second dioecious species are introduced, fertilisation will take place and hybrid oosperms result. When the plants grow to maturity it is found that they are intermediate in their characteristics between the two parents. If now the sperms of the first plant are added to the ova of the second, similar hybrids will also be produced and these hybrids are identical with those produced in the first experiment. One might be inclined to think that such a result was only to be anticipated and pass on without further reflection. But this simple observation shows that the characters of the parents are transmitted in the gametes, notwithstanding their minute size ; and further, that these characters are equally represented in the excessively minute sperms and in the larger ova, and that the influence of the sperm on the development of the embryo is as potent as that of the ovum. Hence it is probable that the amount of hereditary material contributed by the sperm is equal to that contributed by the ovum. Owing to the unequal amount of cytoplasm in the two cells it seems highly improbable that the cytoplasm is the hereditary substance and controls the development of the offspring. While the bulk of cytoplasm in the two gametes is very different it may be observed that the bulk of granular material in the nuclei (chromatin) of the two gametes is at least approximately equal. This observation suggested to investigators that the chromatin of the nucleus is the hereditary substance or germ-plasm, and the view is supported by many other considerations, some of which will be spoken of later.

If the chromatin of the nucleus of the gametes is the germ-plasm then we may with equal probability assume that the chromatin of the nucleus of a ispore is its germ-plasm. Wherein then may we expect reproduction by spores to differ from that by gametes? Evidently the spore contains the germ-plasm of one individual only, while the oosperm, or zygote, contains that of two. Offspring developing from an oosperm will be the mean of two individuals so that idiosyncrasies will tend to be averaged out, while the individual peculiarities of the single parent may be looked for in 
the offspring developing from the spore. On the other hand, there is double the chance of variation in the individual which comes from the oosperm. These two results tend in different directions and on the whole we may look for greater numbers of variations in sexual reproduction, while we may expect among the individuals produced from spores wider and more unbalanced differences.

Thus we see that sexual reproduction. possesses certain qualities which are not shown by asexual reproduction and under certain conditions these qualities may well be advantageous, and so have become fixed in certain organisms.

In order to introduce new qualities by sexual reproduction it is evidently advantageous that the sexual cell should originate from plants as widely separated as possible. In stationary organisms like plants this demands that the gametes themselves should be motile. As the organs of motility are cilia the cells must be small and hence the supply of reserve material in the motile cell is limited. A compromise securing enough stored food material for the developing embryo and an introduction into its constitution of the foreign element is secured by the differentiation of the gametes into the large quiescent ovum charged with store materials and the active travelling sperm coming possibly from a distance, or from an individual or organ possessing a different genealogy from the ovum.

\section{PRACTICAL WORK.}

Cut transverse sections of the receptacles of Fucus platycarpus. Place these sections in a staining block containing salt water. Select out the thinnest and mount in a drop of sea-water and cover. Notice how the cell-walls of the medullary cells swell up and become gelatinous. Sketch the elements of the various tissues, cortex and medulla. Observe and draw the conceptacles. Good complete median sections of the conceptacles are hard to obtain and usually must be built up from many fragments. The walls, the hairs, the antheridia and the oogonia are easily identified. The antheridia are on shrub-like hairs formed of branching filaments of cylindrical cells which support the sac-like ovoid antheridia with coarsely granular contents, which are yellow when mature. The oogonia are the most prominent objects in the preparation. The mature oogonia show the eight rounded ova enclosed in the transparent membranes. The ova are olive-brown. Stages between the first beginnings of the oogonium up to the formation of the ova are easily found and should be drawn.

Ripe sperms and ova are often found in these preparations or they may be collected by immersing mature receptacles in a small quantity of seawater. In this manner the act of fertilisation and the first stages in the development of the embryo may be studied.

The dimensions of the ova and sperms should be estimated by means of the Ghost-micrometer, 


\section{LECTURE XV.}

Polysiphonia is one of the Red sea-weeds. In general its species are small and inconspicuous, being at most a few centimetres in height, but it makes up for its diminutive size by its beauty of colour and of structure.

Our study will be of Polysiphonia fastigiata which is always found growing on one of the Brown sea-weeds (Ascophyllum nodosum), a large Fucus-like plant with a narrow frond and large and conspicuous bladders. Polysiphonia appears like tufts of coarse dark red-brown hair over this plant. Although it always grows on this plant, apparently it uses the brown weed only as a support and is not parasitic upon it. Technically it is an epiphyte.

When submerged in water Polysiphonia shakes itself free and appears as a delicate filiform plant, of an exquisite deep rose colour, the branches of which taper out into great fineness. Examined microscopically each branch is seen to be cylindrical and composed of segments. Each segment is made up of an axial cylindrical cell surrounded by about eighteen prismatic cells which form a cortex round it. All these cells are of the same height. The axial cell, which is somewhat bulged at its equator, and so somewhat barrel-shaped, has a diameter about equal to its height. The radial sides of the prismatic cells are as high as the axial cell but measure radially only about one-fourth or one-third their height. Owing to their wedge-like form their outer tangential wall is about twice as wide as their inner wall and its width equals about $\frac{1}{4}$ of its height. The upper and lower ends of the cortical cells are roof-shaped-the ridge running radially in the stem, and fitted between the adjacent ends of the cortical cells of the segment above and below. Owing to this bevelling of the ends of these cells their outer tangential walls are elongated hexagons when viewed from outside. Often a slight spiral twist of the branch imposes on these cells a distortion, which relieves the regularity of construction from geometrical severity. The distinct outlines and graceful proportions of the cells and their 
symmetrical arrangement confer on this branch an architecture which is strikingly beautiful.

The walls of these cells are comparatively thick, and against their inner surface is the usual layer of cytoplasm containing one or more small nuclei (invisible till stained) and countless chromatophores. These latter are very minute and so closely packed together that the cytoplasm appears almost uniformly coloured with them. They have a rose colour, due apparently to an intense red pigment, which completely masks the presence of a green colouring substance. This latter is plainly seen when
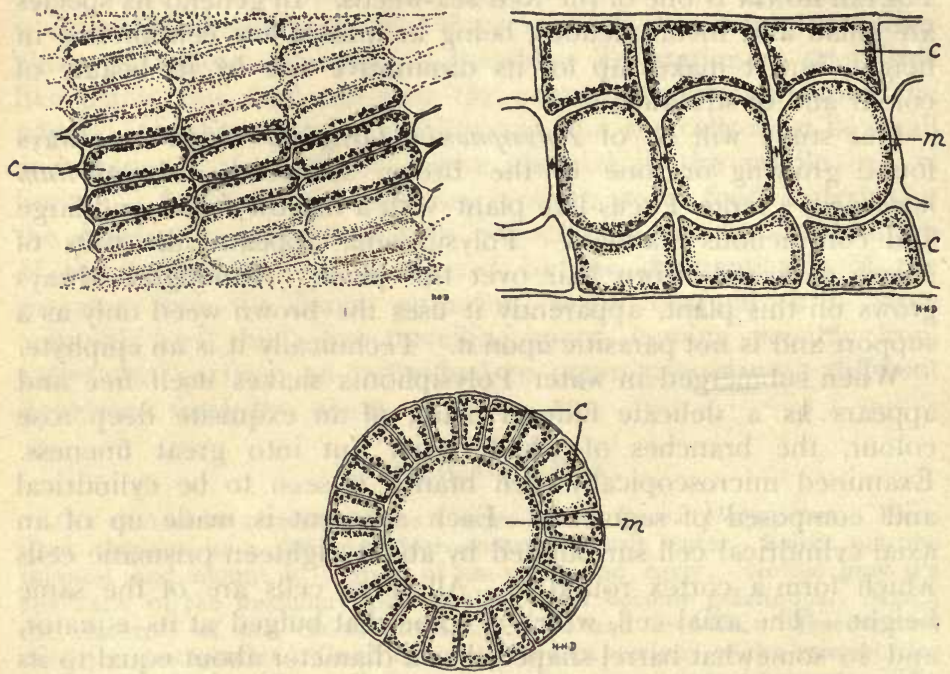

FIG. 20.-Polysiphonia fastigiata, branch above on left, sketch drawn when microscope is focussed on the near side, on the right, when the focus is transferre $t$ to the median plane; below is a reconstructed transverse section. All $\times \mathrm{I}_{50}$. $c$, cortical cell $m$, medullary cell.

the red pigment is removed by alcohol or hot water. The green colour is due to the presence of chlorophyll, possibly in its two forms $a$ and $b$ together; very probably with a certain amount of xanthophyll and carotin. In the cytoplasm are also often seen granules of a substance resembling starch and crystals of protein. The contents of the axial cells resemble those of the cortical cells save that the chromatophores are not so densely crowded in their cytoplasm.

As we pass in the examination of one of the branches of 
Polysiphonia from its base out to its end, we find that it bears two kinds of lateral shoots. The first are just like the main axis possessing a cortex and a chain of axial cells; the second are filamentous, being a single row of cells tapering to a point, and supporting a few similar filamentous branches. The name branches may be reserved more properly for the former; the latter are described as hairs. The extreme end of a branch is rounded, being occupied by a small hemispherical cell. Immediately next to this and below it are found several discshaped cells. These have arisen as segments cut off from the base of the hemispherical cell, and each gives rise to the cells of one whole segment of the stem. At a short distance back from this short apical cell longitudinal divisions are formed in the disc-shaped cells cutting off the cortical cells from the axial cell. The first division which is formed partitioning the discoid cells from the apical cell is not absolutely complete, but the transverse wall is left perforated by an exceedingly minute pore, through which a fine thread of protoplasm passes connecting the discoid cell with the apical cell. When the next segment is cut off a similar protoplasmic connection is formed. Hence the whole series of axial cells in a branch are connected by these fine protoplasmic strands. They may be often clearly seen by focussing deep into the branch. In the same way the partitions of cellulose separating the cortical cells from the axial cell are perforated and protoplasmic connections are left binding the cortical and axial cells of a segment. It is evident that this method of development cannot provide protoplasmic connections between the cortical cells of adjacent segments. But such connections are to be found in the older parts of the branch. They are produced in a very peculiar manner. The nucleus of a cortical cell moves towards one end and there divides in two. One of the nuclei thus formed moves still closer to the end and becomes separated off into a very small cell. The partition wall, however, is perforated, and so the cytoplasm of the cortical cell and its tiny fragment are continuous. The cytoplasm of the fragment now grows out towards the nearest cortical cell of the adjacent segment, dissolving its way through the barrier of cellulose. When the channel is complete and connection is made with the cytoplasm of the cortical cell of the neighbouring segment the nucleus of the fragment passes through the channel and enters the adjacent cortical cell. The fragment now shrinks in size and only a fine thread of protoplasm, with a slight enlargement upon it, 
persists. Thus the protoplasm of the cortical cells of the successive branch segments become connected and the number of the nuclei in these cells is augmented.

Branches are formed close to the apex. They arise by the formation of a lateral bulge on one of the disc-shaped segments beneath the apical cell. This becomes cut off from the discshaped cell and acts as a hemispherical cell. If longitudinal divisions do not quickly supervene it becomes a hair : if they occur it is called a branch. No chromatophores are found in the cells of the hairs.

Carbohydrates are formed in the cortical cells of the branches by the chromatophores which contain chlorophyll and accessory pigments. They are often stored there, and in the axial cells, in the form of the starch-like bodies already mentioned. The necessary salts, especially nitrates, and water appear to be absorbed all over the surface; possibly the hairs are specially effective in this absorption. The primary protoplasmic connections of the axial cells convey proteins elaborated from the carbohydrates and mineral supplies to the apical cell and the growing region immediately below it. The slower growth of the basal parts and the repair of their waste is provided for by the radial connections and the secondary connections of the cortical cells. The small size of the plant renders the development of a special conducting tissue such as is found in Fucus unnecessary. For respiration Polysiphonia utilises the oxygen dissolved in the sea-water. It is therefore aerobic.

Polysiphonia has two very distinct methods of reproduction, an asexual and a sexual process. They are of great interest as elucidating the life-history of higher plants.

Asexual reproduction is effected by means of tetraspores. These, as their name indicates, are formed in fours in a mother-cell-the tetrasporangium. The tetrasporangium arises as follows : one of the cortical cells of a segment near the apex divides longitudinally into an inner and an outer segment. The inner segment divides transversely into a large upper cell and a small basal one. The upper one grows and swells into a large spherical cell, which is the tetrasporangium. Its nucleus divides into four, and the cytoplasm cleaves into four masses each containing one of these nuclei. These cells are the tetraspores. They are arranged tetrahedrally in the sporangium. At first, owing to their mutual pressure, they each have three flat pyramidal faces. Later, as the wall of the sporangium stretches they become spherical. They then escape by the breaking up of the branch in which they are formed. 
Usually a large number of tetrasporangia are formed in a branch which becomes distorted and nodular as they mature.

The tetraspores on their escape float passively in the water. When they settle on a suitable support they adhere to it and form round themselves a cell-wall. The wall in contact with the support is sucker-like and fixes the germinating spore. A transverse division establishes an apical cell and immediately initiates the characteristic growth of the plant.

The sexual reproduction of Polysiphonia presents many features of interest. The sperm cells are not provided with cilia; they are developed in large numbers in special organs-the antheridia, which arise in the same manner as the hairs. Unlike these, however, at an early stage one of the branches of the hair develops a cortex. From the cortical cells numbers of small cells are developed. They are produced like the cells of short bifurcating filamentous hairs, but so closely crowded together that they form a compact tissue-like mass. Their terminal cells form the superficial layer of the club-shaped antheridium, which is attached to the axis by the basal part of the hair. When mature, the protoplasmic contents of each superficial cell emerges as a naked cell and is borne about by the currents in the water.

The female gamete is called the carpogonium. It too is the terminal cell of a lateral hair, which is developed from a cortical cell of a branch. Immediately after the differentiation of the cortex of a filiform branch, one of its cells divides longitudinally into an inner and an outer segment. The outer segment subdivides and develops into a short filament of four cells-the carpogonial hair (often called branch), which turns upwards so as to be almost parallel to the row of axial cells. The terminal cell of this branch produces a long process while its base is enlarged so that the cell may be compared to a flask with a long and very slender neck. This cell is the carpogonium. The filiform process is called the trichogyne. The inner segment of the cortical cell now divides transversely into two, one of these, the upper portion, is called the auxiliary cell. It is approximately at the same level as the base of the carpogonium. Meantime the cortical cells at each side of the carpogonial hair develop and subdivide, giving rise to short filaments of cells which grow round the carpogonial hair, envelop it, and converge on its outer side. These filaments cohere together and form a spherical receptacle coating over the carpogonial hair while they allow the trichogyne to project through a minute gap between them. This whole structure, attached by the base of the branch on which it has developed, is called a procarp. Usually 
other bifurcations of the branch are also attached to the common base.

When the sperms emerge from the superficial cells of the antheridium they are carried by currents in the surrounding water, and apparently their numbers are sufficient for chance alone to secure that at least one will reach almost every trichogyne. Once a sperm touches a trichogyne it adheres to it and the thin membrane of the trichogyne dissolves at the point of contact, and the protoplasm of the sperm forms connection with that of the carpogonium. The nucleus now moves out of the sperm and passes
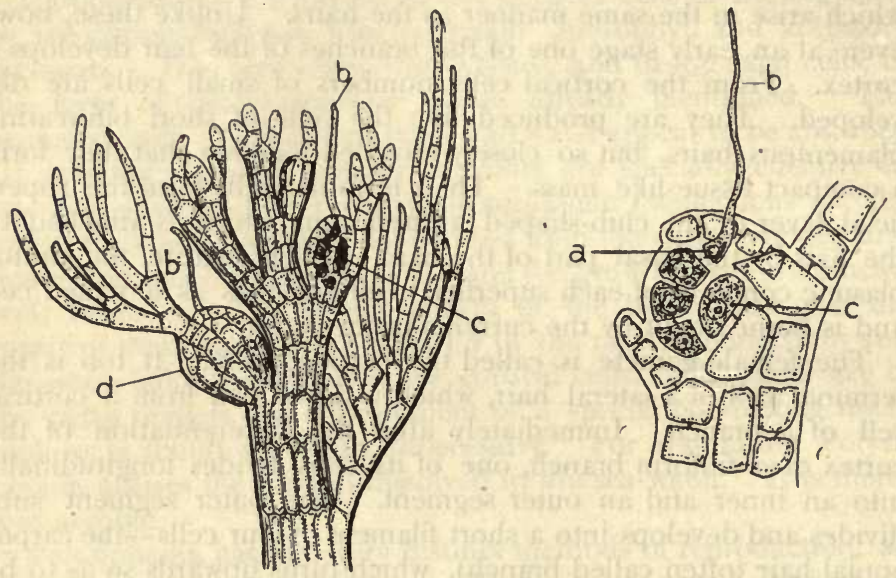

FIG. 2I.-Polysiphonia. On the left, ends of branches and cystocarps, $\times$ I Io. On the right, $a$, procarp, longitudinal section, $\times 600$. $a$, carpogonium; $b$, trichogyne; $c$, auxiliary cell; $d$, young cystocarp. (Partly after Oltmanns.)

down along the trichogyne and fusing with the nucleus of the carpogonium forms the oosperm.

The cytoplasm of the carpogonium is stimulated by this fusion to develop a papilla, which grows toward the adjacent auxiliary cell. The nucleus of the oosperm divides and one segment of it moves into the papilla. The latter continues its growth through the cell-wall and finally penetrates into the auxiliary cell and unites the cytoplasm of the carpogonium with that of the auxiliary cell. Along this connecting bridge the segment of the oosperm nucleus passes and takes up its position in the auxiliary cell. The process reminds one strongly of the formation of the 
secondary protoplasmic connections among the cortical cells. Once in the auxiliary cell the segment of the oosperm-nucleus divides repeatedly and some of its segments move into buds formed on the surface of that cell. These buds become the origin of short filaments, the terminal cells of which enlarge, become ovoid and are called carpospores. The mass of carpospores so formed is enclosed in the receptacle formed by the filaments enclosing the procarp. An opening is left into this fructification or cystocarp, as it is called, at the pole towards which the cortical filaments converge and through it the carpospores are set free when they mature.

Plants bearing tetrasporangia do not produce antheridia or carpogonia. Neither do sexual plants produce tetrasporangia. It is evidently of great interest to ascertain how the two forms of Polysiphonia are related. Observation has brought to light the surprising fact that a plant bearing tetrasporangia never gives rise normally to one bearing tetrasporangia. Similarly a sexual plant never produces from its carpospores a sexual plant. The adage that " like begets like" is strikingly contradicted by the life-history of Polysiphonia.

The carpospores are set free usually in the late summer or autumn. They germinate immediately and give rise to plants which do not attain their full size before the winter. In the following spring they continue their growth and begin the formation of reproductive organs in May, and about June form tetrasporangia. The tetraspores are set free in July and germinate forthwith. The plants arising from them mature quickly, and in August form antheridia and carpogonia. The carpospores arising from these are set free from the cystocarps in August or September, and so the cycle is completed. Inasmuch as the life-cycle is not wholly controlled by seasonal changes, the life-histories of all individuals in one locality do not synchronise with one another, and one often finds tetrasporic plants and carposporic plants out of season; but the calendar given above is that which has been noted in a series of carefully observed cultures. The want of simultaneity, however, in no way interferes with the sequence of the different stages and in no case has it been found that a carpospore gives rise to a carposporic plant. A regular alternation is the rule. A carposporic plant bearing sexual organs, antheridia and carpogonia and producing carpospores, is developed from a tetraspore. While a plant producing tetrasporangia containing tetraspores is the outcome of a carpospore. The line of descent is thus a regular alternation of sexual plants and asexual plants. 
It is of great interest that this alternation of sexual and asexual generations which we have just studied in Polysiphonia, occurs in less precise and clear-cut phases in many simpler and more primitive plants, while it is found firmly established and with unfailing reiteration in the life-history of all the higher types of vegetation. Nothing strictly comparable to it characterises the life-histories of animals. Its occurrence is one of the broad distinguishing characteristics of the vegetable kingdom.

\section{PRACTICAL WORK.}

- Mount a small piece of Polysiphonia in sea-water. Observe that when the branch is caused to roll over by pushing the cover-glass on one side it is evidently cylindrical. Sketch with low power. With the high power make drawings of it at different levels of focus. The first is to be of an external view of a portion of a branch. It will show the outer surfaces of the cortical cells on one side of the branch. Those in the middle line of the branch next the observer will show their complete outer wall, which being viewed perpendicularly will not be foreshortened. The outer faces of those removed from this line, forming the curved surface of the cylindrical stem, are oblique to the line of vision of the observer and appear foreshortened and consequently will have to be represented narrower on the drawing. The number of cells visible on the near side of the branch by focussing at different levels should be carefully noticed. The drawing should also show the manner in which the cortical cells of the adjoining branch-segments interlock. The cell contents of the nearest cortical cells should be recorded and careful search made for the protoplasmic communications between the cells.

The second drawing should represent a median optical section of the branch and should show about three successive axial cells in section and on each side of them radial sections of the cortical cells. Carefully record the dimensions of the cells in both drawings.

Reconstruct a transverse section of the stem. Being a cylinder its transverse section must be a circle. Make a circle having a diameter equal to that of your longitudinal optical section. Within this draw a concentric circle having a diameter equal to that of the axial cell in the longitudinal section. Now subdivide the circumference of the outer circle into as many divisions as there are cortical cells-i.e. double the number recorded in the first drawing. These divisions should equal the width of the middle cortical cells in the first drawing. Draw radii from the points marking these subdivisions on the outer circle to the inner circle. In this way the outlines of the cells will be obtained as they would be seen in transverse section.

Search should be made for the reproductive organs, tetrasporangia, antheridia and procarps in their various stages of development. The carpogonial branches and trychogynes are very difficult to observe. The details of their structure can only be made out by embedding and section-cutting. 


\section{LECTURE XVI.}

IT is a highly suggestive fact that all the simplest and most primitive forms of plant life are inhabitants of water and it is only among the more highly developed that we find those which are so constructed as to be able to support themselves on land. Of the examples which we have discussed so far only the two fungi are adapted to sub-aerial conditions. Many of the fungi, however, are aquatic and even those which produce aerial organs have the greater part of their mycelium continually immersed in a liquid or moist substratum, or are embedded in the watery tissues of their hosts, and the aerial organs are formed only in connection with mechanisms to secure the distribution of their spores by means of air currents. It is interesting to note that it is in the fungi which are thus sometimes partially adapted to sub-aerial life that we find peculiar tubular organs developed for the intracellular transmission of the gametes to effect fertilisation, so that the transport necessary to effect fertilisation is independent of aerial conditions.

The simple body of these aquatic or semi-aquatic plants is not differentiated like that of the higher plants into stem, root and leaves. Being for the most part completely immersed in a fairly uniform medium, the raw materials needed for nutrition may be absorbed all over their surface. Hence no advantage would be obtained in the development on their undifferentiated body or thallus of special areas for the absorption of carbon dioxide and for photosynthesis, or for the absorption of mineral salts. These more simple and undifferentiated plants thus primarily adapted to an aquatic life are conveniently classed together as Thallophyta, a name descriptive of their simple morphology, which is in harmony with the physiological uniformity of the medium in which they live.

Our subject for this lecture-Marchantia polymorpha-is a land plant, but one possessing so many of the characteristics of aquatic vegetation that it seems as it were to illustrate in many ways how a land flora may have originated from aquatic and semi-aquatic plants. 
Its thallus-for its body is not differentiated into stem, root, and leaves - is flat and ribbon-like, and grows over moist soil. It is dark green in colour and shows many bifurcations. The ends of the branches show deep indentations and their edges are irregular and slightly crinkled. The indentations at the ends of the branches mark the position of the growing regions of the thallus. Passing back from these to the older parts a shallow groove may be seen which marks the position of the mid-rib. When the growing region divides to form a bifurcation naturally the mid-rib divides too. If closely observed the surface of the thallus on each side of the mid-rib may be seen to be marked out into very small rhomboidal areas, and in the middle of each of

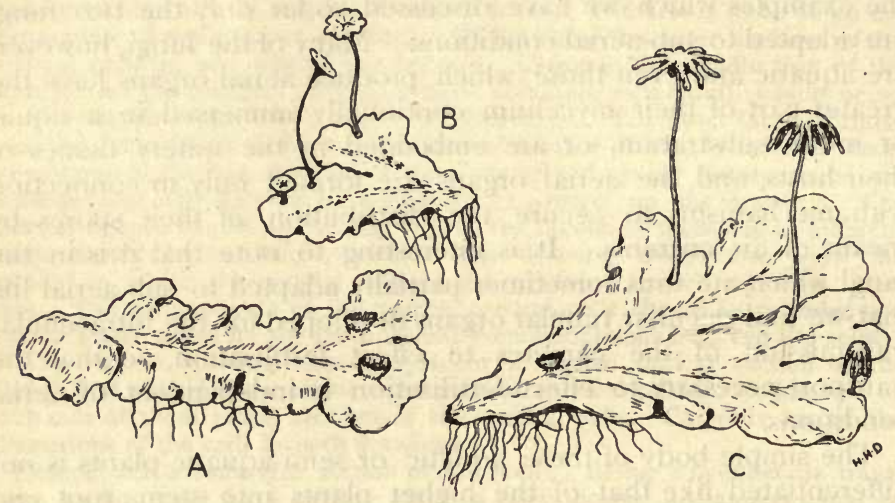

FIG. 22.-Marchantia polymorpha. A, plant with cupules, winter condition; $\mathrm{B}$, plant with antheridiophores; C, plant with archegoniophores.

these areas a minute red speck is seen. These areas mark the position of air-chambers in the thallus immediately below the superficial layer of cells, and the speck is a short tube or flue perforating this layer and ventilating the chamber below.

If the thallus is raised from the soil and its lower surface studied, it will at first appear covered with the adherent soil particles. When they are removed the mid-rib is seen as a low ridge running medianly in the thallus; from the under surface spring countless fine colourless hairs which penetrate among the particles forming the surface of the soil and fix the plant to them. They are called rhizoids. There are also numbers of irregular membranous scales attached to this surface of the plant on either side of the mid-rib. 
The minute structure may be best made out from a transverse section of the thallus. Under the low power this section shows that the mid-rib of the plant is much thicker than the edges. The upper region of the section is green owing to the presence of tissues containing much chlorophyll, the lower two-thirds to seveneighths is colourless or at least not coloured green. From the lower surface spring numbers of tubular structures, the rhizoids, and what in the section look like chains of cells, which are the transverse sections of the scales.

With a high power further details may be made out. The cells of the upper superficial layer are small and almost square in crosssection. They have thin walls and their protoplasmic lining con-

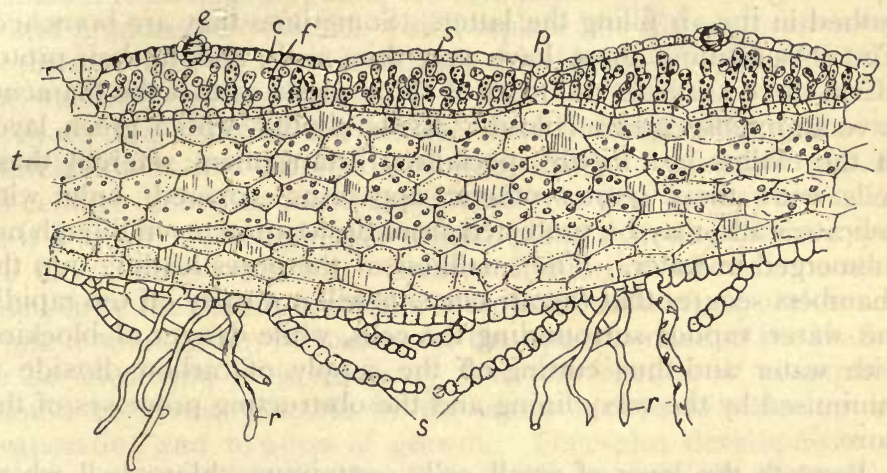

FIG. 23.-Marchantia polymorpha, thallus transverse section, $x$ roo. $c$, cuticle; $e$, ventilating pore leading into air-chamber; $p$, partition; $r$, rhizoids; $s$ (on upper surface), superficial layer; $s$ (on under side), scales; $t$, layers of large cells.

tains small ovoid chloroplasts embedded in it. They are uniform in size, and fitting closely together, they form a continuous membrane covering the upper side of the thallus. Here and there the section cuts through a ventilating pore or the cells forming the short tube or collar surrounding it. One can see that the collar consists of four tiers of narrow cells. Two of these tiers are raised above the superficial layer and two are below it. Inspection of the collar in a surface section of the thallus shows that each tier is an oval ring composed of four cells, so that the collar surrounding the pore is built up of sixteen cells in all. The four cells of the lowest tier have each a blunt tongue-like bulge upon them more or less obstructing the aperture of the pore. These 
obstructions and the inside of the wall of the pore are coated with a waxy material which is not easily wetted, so that a drop of water finds it very difficult to enter by the pore. Immediately below the superficial layer are the air-chambers of the thallus. They are separated from one another by small vertical walls one or two cells thick resting on the floor of these chambers. The cells forming these walls and the floors of the chambers are similar to those of the superficial layer in size and form, and like them they contain chloroplasts. The cavities of the chambers are partially filled with dark green cells. Examination of these shows that the cells are somewhat barrel-shaped and they are grouped together in short filaments 3 or 4 cells in length. These filaments grow up from the floor of the chamber and extend into its cavity, being bathed in the air filling the latter. Sometimes they are branched. The cells forming them have very thin walls, and in their protoplasm there are great numbers of chloroplasts so that the filaments have an intense green colour. Evidently this whole green layer of the thallus is a highly specialised arrangement whereby these cells with their photosynthetic apparatus, covered only with delicate walls, may be protected from desiccation, even though not submerged in water. The smallness of the pores leading into the chambers secures that the air currents will not carry off too rapidly the water vapour surrounding the cells, while danger of blocking with water and thus cutting off the supply of carbon dioxide is minimised by the waxy lining and the obstructing processes of the pore.

Beneath the layer of small cells containing chlorophyll which form the floor are several layers of large cells with little or no chlorophyll. These cells often contain a certain amount of starch and always a large watery vacuole. Their function seems to be to hold a supply of water for the green cells and to store the material these have formed in photosynthesis. Their walls are relatively thick, sometimes they have narrow groove-like marks upon them which are thin places on the walls; these are called pits. Probably the latter facilitate the movement of liquid from one cell to another.

The lower surface of the thallus is formed of two or three layers of small irregular cells. . Their walls are often coloured violet or brown, they are without chlorophyll. The rhizoids are seen to be outgrowths from some of these cells at the surface. They are of two kinds; rhizoids with straight tubular walls, smooth on the inside as well as the outside, and others with constricted walls furnished on their inside with numerous peg- 
like ingrowths projecting into the cavity of the cell. Very little protoplasm can be seen in any of these rhizoids, they are chiefly filled with water and their function, in addition to fixing the plant, seems to be drawing water from the soil to the plant. Of course this water will be a dilute solution of salts and hence these rhizoids supply the plant not only with water but also with all the other constituents it requires as food, except carbon. If the demands of the plant exceed the supply from the soil evidently the walls of these flexible tubes will tend to collapse. Probably the pegs on the inner walls are to guard against complete collapse which would close these channels of supply.

A curious phenomenon is often seen in these rhizoids. When a rhizoid loses its protoplasm, the cells adjoining its base grow into it and produce new rhizoids which replace the old one; as many as three or four of these secondary rhizoids may be found within the wall of the primary one.

From this short sketch we see: that although the thallus of Marchantia is not differentiated into distinct vegetative organs, its cellular differentiation is considerable, so that we may recognise in it a much more highly developed plant than any of the types we have hitherto studied. Greater division of labour is necessitated since the thallus is seldom completely submerged, and hence while it would often be useless for the upper surface of the thallus to be absorbent, it is necessary that the function of absorption should be always possible by the under surface to make up for evaporation and to allow of growth. Then also development of the storage tissue enables the plant to tide over periods of drought, but the thickening of the thallus which is necessary to secure this end cuts off the lower surface from the light and necessitates the limitation of the photosynthetic tissues to the upper surface. The dorsal position of the thin walled photosynthetic cells necessitates protection from evaporation: at the same time free access of the air and its carbon dioxide is required. The chambered structure of the upper tissues with pores in the roofs of these chambers meets both these requirements.

Marchantia has two prolific methods of asexual reproduction. The first is a purely vegetative process and depends upon the short-lived nature of the vegetative cells. These cells appear normally to have a span of life of about one or two months. As the new cells are formed at the tips of the branches, the cells at a short distance from these tips become senescent and die. As soon as the cells forming the bifurcation uniting two branches are involved in this senile decay the two branches become independent 
of one another and each forms a separate plant. This forms another example of the frequent-though at first sight paradoxical -connection of death with reproduction.

The second method of asexual reproduction is also very prolific. It is carried on by means of gemmæ, which are small lensshaped masses of cells about a millimetre in diameter, and when detached from the plant grow into new plants. These gemmæ are found attached to the bottom of small cup-shaped organs (cupules) developed on the middle of the thallus. The cupules have a colourless serrated margin. Each gemma is attached to the bottom of the cupule by a short stalk. It arises as a twocelled hair. The distal cell enlarges and subdivides and thus produces a lens-shaped mass of cells. There are two indentations in the periphery diametrically opposite to one another. These become the first growing points of the young plant. When the gemma has attained its full size it is set free by the breaking of the stalk formed of the basal cell, and if it finds itself in a suitable position, certain of its superficial cells grow out and develop into rhizoids. The rhizoids develop from specially large cells of the gemma which do not possess chlorophyll. They are found on both sides, but only those develop which have a chance of being of use to the young plant. This purposeful development has been shown to be due to the response of these cells to three kinds of stimuli. Thus moist air and a positive gravitational stimulus evoke their development, while light and a negative gravitational stimulus inhibit it.

The response then to these stimuli secures that the rhizoids of the young plant are normally developed on that side which is in contact with the ground. As soon as these rhizoids begin their absorptive functions the growing points in the two depressions on the margin of the gemma become active and they soon convert the gemma into a small thallus. At first there is no differentiation in the gemma into dorsal and ventral aspects, either side can develop according as external stimuli direct its growth: but soon a certain point is reached in the development when the dorsiventrality of the tissues of the young plant is established and then the dorsal side loses the power of developing rhizoids, etc., like the ventral aspect, and the ventral aspect loses the power of developing as the dorsal. The cells of the gemma, which have developed in a vertical plane while in the cupule, are not differentiated in their responses to these stimuli, but those formed from the growing regions, when the gemma is in a horizontal position, respond differently to the same stimuli. Hence we must assume 
that different characteristics are dealt out to the different cells as they are formed in the growing region in response to external stimuli, or that these stimuli differentially inhibit or develop potentialities in the dorsal and ventral cells.

\section{PRACTICAL WORK.}

Sketch both aspects of the thallus of Marchantia polymorpha, magnifying it 4 to 8 diameters.

Cut off a thin piece from the upper surface, mount and examine. Sketch the rhomboidal areas and the central pore, using the low power.

Make a transverse section and mount. This requires to be very thin to show the structure of the dorsal tissues satisfactorily. A sketch should be made with the high power of the green layer, showing the cells round the pore, those of the roof, floor and photosynthetic filaments of an air-chamber.

Sketches should also be made of the cells of the storage tissue and of the two kinds of rhizoids.

Specimens showing cupules and gemmæ are easily obtained in May and June. The vegetative structure of the thallus may be studied at any season of the year. 


\section{LECTURE XVII.}

Asexual reproduction by gemmæ in Marchantia usually takes place in the autumn and spring, while sexual reproduction is commonly observed during the summer. Hence it is that the sexual organs are not commonly found on the plants with most cupules. Plants in the sexual stage are very easily recognised. They are furnished with very remarkable vertical branches. These branches are of two kinds, antheridiophores and archegoniophores. Both kinds seem never to occur on the same plant. They are developed at the growing region and form a direct continuation of the mid-rib; but they bend vertically upwards, using an indentation at the end of the horizontal branch to emerge from underneath the margin of the thallus. The margin of the thallus often by subsequent growth closes round them so that to casual observation they appear as growing up through the thallus.

The antheridiophore has a whitish stalk about $\mathrm{r}-3 \mathrm{~cm}$. long, which supports a disc-shaped top. The stalk is roughly prismatic, having five faces. Two of these faces are bowed outwards and three are grooved. The grooves on two are very deep and the sides close over them, so that two vertical tubular channels are formed running down the length of the stalk. At the upper end the stalk spreads out to form the disc. Eight distinct lobes on the margin of the disc show that we have to do with a system of branches, each lobe representing the apex of a branch. The middle of the disc is thick and massive and the margin is membranous. Its surface resembles the upper surface of the thallus and is provided with numerous air-chambers, each with its central pore. In addition to these pores the upper surface of the disc is studded over with many dark purple spots which are most numerous along the low ridges which run radially out to the lobes of the margin. These spots indicate the openings of narrow canals leading down into deep rounded cavities in the antheridiophore. The cavities are lined by a layer of cells, the walls of which are coloured a deep purple. Each cavity is almost completely filled with an egg-shaped body-the antheridium-attached 
to the base of the cavity by a slender stalk. The antheridium is a thin-walled sac containing countless sperm-cells. When the sperms are ripe, if water finds its way to the upper surface of the antheridiophore it is drawn through the narrow canal, and passing into the cavity leads to the rupture of the antheridium. The sperms are discharged as a milky droplet and are driven out on the surface of the antheridiophore. They are threadlike and very

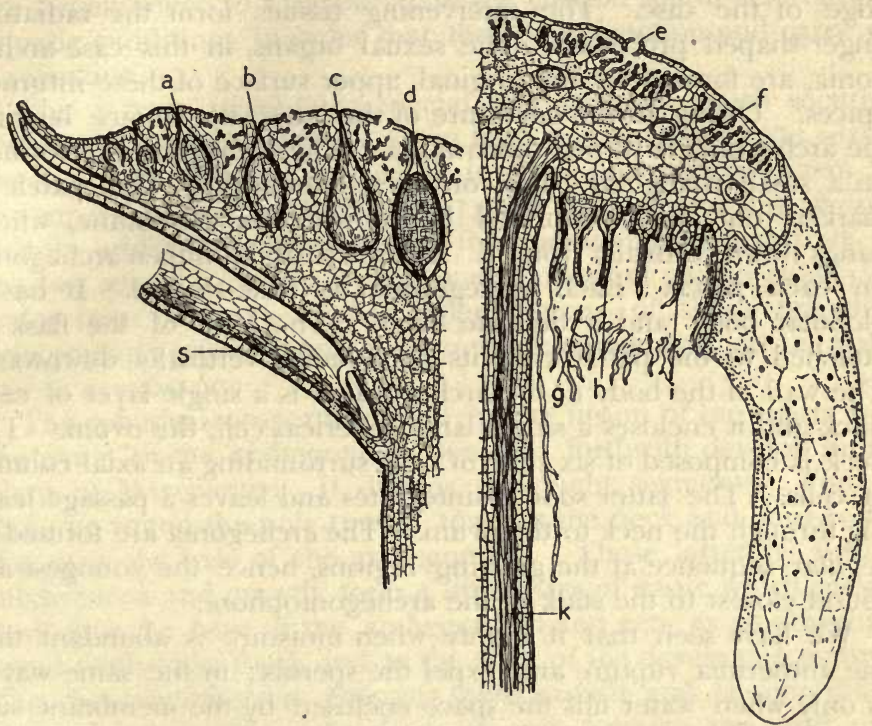

FIG. 24.-Marchantia polymorpha, longitudinal section of antheridiophore on left and of archegoniophore on right, $\times 25$. $a$, young antheridium cut in median plane; $b$, outside of young antheridium; $c$, antheridial cavity showing the cells which line it ; $d$, median section of older antheridium; $e$, ventilating pore; $f$, photosynthetic filaments; $g$, membrane surrounding archegonia; $h$ and $i$, archegonia; $k$, rhizoids in one of the longitudinal grooves of the stalk; s, scale on antheridiophore. (Partly after Kny.)

minute ; they possess two long cilia. While still in the antheridium the sperms appear as a ring or flattened spiral, but when they are set free they assume the shape of a cork-screw blade. They are carried by dew or rain falling on the upper surface of the antheridiophore over its margin and are, in all probability, conducted to the soil by rhizoids which are attached to the under surface of the disc, and travel down the stalk by the two tubular channels already mentioned. 
The archegoniophores are similar to the antheridiophores in their relation to the thallus. They are also differentiated into a stalk and disc; but the stalk is usually longer and the disc is subdivided into finger-shaped processes. Like the antheridiophore the archegoniophore is a branch-system, but the growing tips very early turn downwards and inwards towards the top of the stalk, so that their positions are marked by deep indentations in the edge of the disc. The intervening tissues form the radiating finger-shaped processes. The sexual organs, in this case archegonia, are formed on the original upper surface of these inturned apices. Owing to the curvature of the latter, the surface bearing the archegonia is turned downwards. The archegonia are formed on a small triangular patch on each branch-tip. This patch is marked out and surrounded by an irregular membrane, which hangs down vertically from it. There are about fifteen archegonia on each patch. Each archegonium is flask-shaped. It has a globular body and a delicate neck. The base of the flask is attached to the patch while its neck hangs vertically downward. The wall of the body of the archegonium is a single layer of cells thick and it encloses a single large spherical cell, the ovum. The neck is composed of six rows of cells surrounding an axial column of cells. The latter soon disintegrates and leaves a passage leading through the neck to the ovum. The archegonia are formed in regular sequence at the growing regions, hence the youngest are found closest to the stalk of the archegoniophore.

We have seen that it is only when moisture is abundant that the antheridia rupture and expel the sperms; in the same way it is only when water fills the space enclosed by the membrane surrounding the archegonia that the disintegrating column of cells in the neck of the mature archegonium swells up and opens the way up to the ripe ovum. These disintegrating cells give rise probably to some soluble substance which acts chemotactically on the sperms; for the latter have been observed to crowd round the neck and swim towards its opening.

The two longitudinal grooves of the stalk of the archegoniophore are like those of the antheridiophore traversed by bundles of rhizoids which take their origin near the outer end of the finger-shaped rays of the disc. Thus at their base they pass close by the membrane surrounding the archegonia and put this tiny reservoir of water in connection with the water of the soil. The sperms conveyed there by the rhizoids of the antheridiophore may be supposed to find themselves under the orienting influence of the dilute chemotactic substance liberated from the archegonia. 
The different grades of its concentration occurring in the grooves of the archegoniophore lead the sperms on among the rhizoids until they reach the archegonial patch. There they are set free among the archegonia and are attracted to an archegonium, the neck of which has just opened. One passes in and fuses with the ovum.

Thus it is evident that while the vegetative parts of this plant are largely suited to a terrestrial life the ciliated sperm-cells require aquatic conditions in order that they may function and carry on fertilisation.

This is only possible by special arrangements which secure a water connection between the two branches supporting the sexual organs. The development of an internal conducting system by the superficial rhizoids is a point of great interest, and it is obvious that in addition to transmitting the sperm-cells, the rhizoids of the two sexual branches must play an important part in conveying water from the soil to the upper regions of these branches, bringing up supplies needed in growth and making good the water lost in evaporation.

The spherical oosperm formed by the fusion of the sperm with the ovum in the archegonium does not forthwith develop into a plant of Marchantia. It divides into eight segments. Four of these lie round the pole turned towards the neck and four round that next the base of the archegonium. These latter by further subdivisions and growth, form a little mass of tissue which presses itself into the base of the archegonium and acts as an absorbing organ (called the foot), and as the stalk of the developing embryo. The distal hemisphere enlarges more rapidly and is soon differentiated into a thin outer layer of cells forming the wall of a capsule and containing a vast number of polyhedral cells fitting closely together. ${ }^{\circ}$ Some of these cells elongate rapidly and develop tubular walls tapering to each end. Their protoplasm disappears after it has deposited spiral thickenings on the inside of the cellwalls. These are elaters. The others are completely filled with protoplasm. Their nucleus is large, and soon divides into four. Round the four resultant nuclei the cytoplasm separates, and so four globular cells (spores) are formed simultaneously in each mother-cell. They are arranged tetrahedrally in the mother-cell and are slightly flattened on three faces by mutual pressure. The outer surface of the walls of these spores is covered with a finely granular deposit from the remains of the protoplasm of the mother-cell.

During the development of the embryo into the spore-capsule, 
the stalk and the foot, the archegonium is stretched and keeps pace with the growth of the embryo within. Outside the archegonium a collar of tissue grows up round it and arches over it and finally the stalk of the archegoniophore elongates considerably, so that it becomes 3 or $4 \mathrm{~cm}$. long. Evidently the effects of fertilisation are not limited to the ovum but they are transmitted to the surrounding tissues.

When the spores and elaters are ripe within the capsule, or sporangium, the stalk elongates and shoves the sporangium out through the top of the archegonium, and out of the in-arching collar, so that it hangs down free and uncovered from the underside of the archegoniophore. At first the protruding sporangium looks like a dark green knob. Later it becomes yellow in colour and splits at the top with 4-6 radial clefts. The mass of spores and elaters, which were apparently in a state of compression,

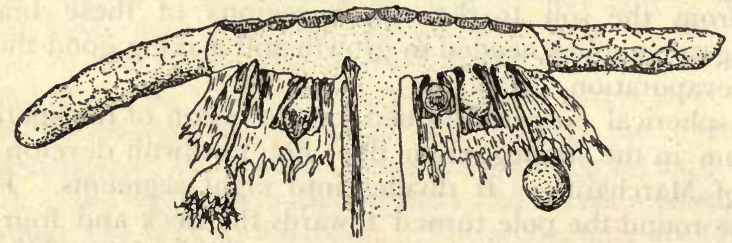

FIG. 25.-Marchantia polymorpha, top of archegoniophore, vertical section, $\times 8$. Fertilised archegonia with embryo-sporophytes in different stages of development are seen.

expands and a large number of spores are liberated. The remainder are retained in the tangled mass of elaters, which adheres to the base of the sporangium, and are shed out in dribs and drabs by the hygroscopic curling and uncurling of the elaters. These motions of the elaters are due to the unequal expansion of the cellulose wall and of the spiral bands of the elaters when imbibed with water. Hence motion must result, when they lose water to a dry, or absorb water from a moist atmosphere. The whole apparatus acts like an intermittent censer or pepper-pot, shaking out small quantities of spores at a time. The height of the archegoniophore facilitates this scattering, so that the spores will not fall out on the soil in a compact mass.

Thus we find an advantage in the elevation of the archegonia in the archegoniophore which compensates for the difficulty that elevation introduces in the way of fertilisation. The spores on reaching the ground germinate immediately and produce a small 
filamentous plant. Divisions are soon developed which transform it into a plate of cells attached to the soil with rhizoids. Growth and differentiation proceed till a diminutive plant of Marchantia is formed.

Comparison of the life-history of Marchantia with that of a simpler organism like Fucus brings out a striking difference. The oosperm of Fucus grows and differentiates directly into an individual which resembles the parents which produced the ovum and sperm. This is not the case with Marchantia. The fertilised ovum grows into the small structure composed of sporangium, stalk and foot, which develops in the archegonium on the archegoniophore. The growth of this structure takes place at the expense of the materials in the archegoniophore, which are absorbed and transmitted to it by the foot. This spore-producing apparatus is entirely dependent and parasitic on the archegoniophore. Plants, like the parents which produced the ovum and sperm, are only formed when the spores germinate and develop. Compared with Fucus the spore-producing apparatus is a new structure introduced into the life-history, and without parallel in the life-history of the simpler plant. The plant which produces the gametes or sexual cells is appropriately called the gametophyte, while the individual which develops from the oosperm and produces spores is called the sporophyte. The complete life-cycle of Marchantia comprises an alternation of these two phases. The first phase is the gametophyte. It consists of the creeping bifurcating thallus, which, absorbing water and mineral salts from the soil by its rhizoids, constructs organic substances by photosynthesis in its green tissues. For reproduction it forms archegoniophores and antheridiophores, producing archegonia and antheridia with their ova and sperms. The second phase is the sporophyte, much simpler in structure, composed of the sporangium, its stalk and foot, and wholly parasitic on the gametophyte.

Evidently such a life-history is more comparable to that of Polysiphonia. In the latter we have a somewhat similar alternation of carposporic phase-the gametophyte-with the tetrasporic phase-the sporophyte.

Another point of difference between the true thallophytes, which, as we have seen, are primitively aquatic plants, and the representatives of terrestrial plants like Marchantia, is to be found in the structure of the sexual organs. The gametangia or receptacles of the sexual cells in the former are bounded by the cell-wall of a single cell within which the gametes develop. In the higher types the gametangia are multicellular and are built up, like the 
antheridia and archegonia of Marchantia, of a large number of cells. The structure of the archegonia is very uniform in large groups of the higher plants and differs very strikingly from that of the oogonia of the algæ. Hence it is sometimes convenient to speak of the groups of the land flora which are characterised by this special form of female gametangium as the Archegoniates. Marchantia is one of the Archegoniates.

\section{PRACTICAL WORK.}

Examine the archegoniophores and antheridiophores with a simple lens. Make sketches of them from various aspects, magnifying them 4 or 5 diameters.

Prepare transverse sections of the stalk. Make sketches showing the longitudinal grooves containing the rhizoids.

Prepare radial vertical sections of the disc of both organs and make sketches illustrating the structure and position of the antheridia and archegonia.

Material collected in May and June will illustrate the development of the antheridia and archegonia. Sections of the antheridiophores will show the mother-cells of the sperms and the production of the sperms. Archegoniophores at the same time will show the development of the archegonia, fertilisation, and the early stages of the sporophyte.

If a drop of water is placed on one of these antheridiophores and watched for a few'minutes with a simple lens, the ejection of the sperms may be easily observed. They appear like minute puffs of milky fluid emitted from the antheridial cavities into drops of pellucid water.

Material taken later in the year, viz. July to October, shows the various stages in the development of the sporophyte up to the dehiscence or rupture of the sporangium and the emission of the spores from it. The structure and movements of the elaters may be observed microscopically by mounting some of them dry and uncovered on a slide. 


\section{LECTURE XVIII.}

Funaria hygrometrica is a very common moss often found on rocks and walls, and especially in sandy situations. It grows in patches, large numbers together forming a close sod. The separate plants in these colonies are about $2 \mathrm{~cm}$. high: each has a wiry vertical stem beset closely with small ovate-pointed leaves. The stem is brown, the young leaves round the top of the stem are green, those lower down are brownish. Rhizoids which are

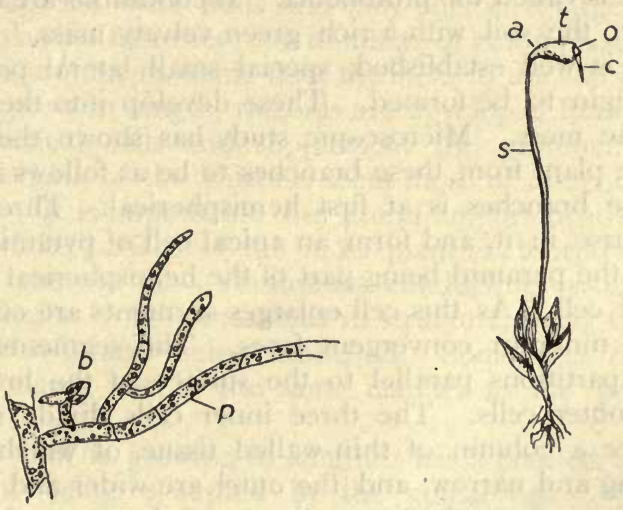

Fig. 26. - Funaria hygrometrica. $\quad p$, a piece of protonema with bud $(b), \times 80$. On right is shown a leafy gametophyte with sporophyte attached, $\times 2$. $a$, apophysis ; $c$, calyptra; $o$, operculum; $s$, seta; $t$, theca. (Partly after Sachs.)

thread-like and of a brown colour are attached to the lower part of the stem and fix it in the soil.

Many of the plants support at the tip of their leafy stem a slender, wiry and twisted stalk, bent at the top. To it is attached a pear-shaped body-the sporangium. At first the sporangium is green, later it becomes yellow and then brown. In its early stages it is usually more or less completely covered with a membranous 
cap, the calyptra. As the sporangium ripens, underneath the calyptra may be seen a tiny brown circular lid, the operculum. Later the calyptra and then the operculum come away and allow a fine brown dust to shake out from the sporangium. The grains of this dust are the spores.

When these spores are shed they are tetrahedral in shape and each is covered with a brown outer wall. They absorb water and become spherical. The brown outer layer breaks and the colourless inner layer of the wall is pushed out as a tubular cell. This grows on and forms a filament of cells, in which there are usually numbers of rounded chloroplasts embedded in the protoplasmic film lying against the inner surface of the cell-wall. As the filament grows it creeps along the surface of the soil and forms large numbers of branches, some of which grow down into the soil. 'The cells of the filament which are exposed to the light are rich in chloroplasts, those which dip into the soil are colourless. The whole branching filament is called the protonema. If conditions are favourable it will cover the soil with a rich green velvety mass. When the protonema is well established, special small lateral pear-shaped branches begin to be formed. These develop into the stem and leaves of the moss. Microscopic study has shown the development of the plant from these branches to be as follows: The end cell of these branches is at first hemispherical. Three oblique partitions arise in it, and form an apical cell of pyramidal shape, the base of the pyramid being part of the hemispherical surface of the original cell. As this cell enlarges segments are cut off from each of its inner or convergent faces. The segments are then divided by partitions parallel to the surface of the branch, into inner and outer cells. The three inner cells divide repeatedly and produce a column of thin-walled tissue, of which the axial cells are long and narrow; and the outer are wider and somewhat shorter. These form the inner tissues of the stem of the moss. The outer cells formed from the first segments of the apical cell give rise to the outer cells or cortex of the stem, and to the leaves. The cortical cells develop thick brown walls and so the cortex becomes a rigid tube encasing the stem and giving it stiffness. As these tissues are being developed and differentiated from the segments of the apical cell, that cell continues to grow and form fresh segments so that growth is continuous. Leaves are formed from certain of the outer segments of the apical cell. By repeated divisions these segments form a small shelf of cells beside the apex. This is a single layer of cells thick and one of the cells centrally placed becomes an apical cell ; by subdivision this gives 
rise on each side of itself to new cells which are added on to, and increase, the length of the young leaf.

The cells immediately behind this apical cell divide longitudinally and form a mid-rib of narrow cells running down the middle of the leaf, while the sides of the leaf remain but one cell thick. The cells of the mid-rib are long and narrow with thin cell-walls and resemble those of the axial strand of the stem, but are not connected with it. The apical cell of the leaf continues its growth and division for a time: when it ceases the leaf continues to grow for a short time by the enlargement of the individual cells forming it ; but that too ceases soon. The mature leaf has an oval outline, and is attached to the stem by a broad base. It has a pointed apex. The cells on each side of the mid-rib fit together closely, they are somewhat elongated, in the direction parallel to the mid-rib, and in form are polyhedral; their walls are at first colourless and subsequently brownish. They contain protoplasm in the form of a film lying against their walls and they enclose a large vacuole. In the protoplasmic film there are numbers of chloroplasts.

At first the protonema alone fixes the stem to the soil but later, as the stem grows in length, rhizoids are developed from some of the lower cortical cells and supplement the protonema. The principal functions of the rhizoids seem those of attachment and reproduction. It is improbable they are of importance in absorption. The whole surface of the moss plant is absorptive. This explains the absence of a continuous conducting system. The rhizoids are similar to the protonema in structure, being composed of cylindrical cells and being branched. Sometimes they give rise to new plants, just in the same manner as the protonema does.

While the stem is growing in length it has a pointed apex, the extreme point being occupied by the apical cell. When the sexual organs are about to be formed, the apex becomes flattened and broader. On the male shoot the leaves round the apex are reddish in colour and on the rounded apex itself are formed a number of short hairs-the paraphyses, and antheridia. The paraphyses are composed of four or five cells attached end to end. The cells at the base are narrow and the distal cells are large so that each hair is club-shaped. The antheridia are not quite so long as the paraphyses. They are elongated sacs on short narrow stalks attaching them to the apex. The wall of the sacs is formed of a single layer of polyhedral cells which contain chloroplasts. The top is closed by a few large colourless cells-the lid-cells. The sac is filled with a vast number of minute cubical cells fitting 
closely together, each with very thin cell-walls and completely filled with protoplasm. The nucleus of each of these spermmother-cells is elongated and continues to grow in length so that it forms a short spiral thread in the outer part of the cell. At this stage if water comes in contact with the antheridium, the swelling up of the mucilaginous material in the base of the antheridium expels the central mass of sperm-mother-cells, bursting open the lid-cells of the antheridium.

Outside the antheridium the sperm-mother-cells continue their development. The cytoplasm forms a coating over the elongated
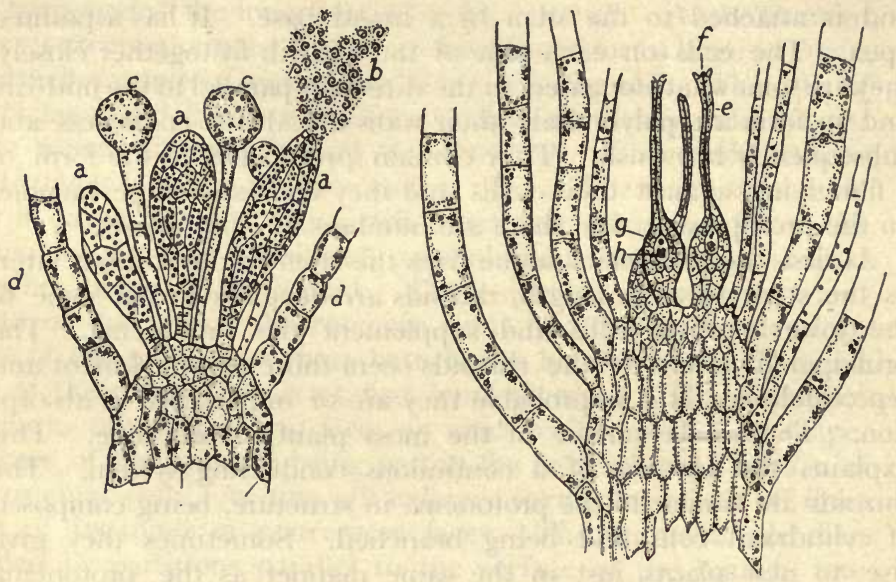

Fig. 27.-Funaria hygrometrica, apex of male branch on left, and of a female branch on the right, $\times 135$. $a$, antheridium; $b$, sperms being set free; $c$, paraphysis ; $d$, leaf; $e$, neck of archegonium; $f$, neck-canal; $g$, venter of archegonium; $h$, ovum.

spiral !nucleus and is produced into two long cilia. By the motions of these cilia, the sperms thus formed begin turning round and round in the mother-cell.

Archegonia are usually found at the apex of a shoot which is a bifurcation of the branch which bears the male shoot. The leaves round this apex are similar to the ordinary foliage leaves and surround a number of paraphyses as well as the archegonia.

The archegonia are in shape like tall narrow flasks on short pedicels. The body is small and the neck long and narrow. The ovum is contained within the body and the passage of the 
neck is occupied by a single column of elongated cells-when these cells disintegrate they leave a canal leading down the neck to the ovum.

The sperm-cells, which by this time have disentangled themselves from the debris of the mass of sperm-mother-cells expelled from the antheridium, are attracted to the necks of the mature archegonia. A single sperm passes through the fluid remains of the neck-canal-cells and makes its way to and fuses with the ovum. The final maturing stages of the archegonia and antheridia can only take place in presence of water and this at the same time supplies a water-way for the transit of the sperms from the male to the female branch. The guidance of the sperms in this passage seems to be due to the formation of a chemotactic substance emitted by the archegonia, developed possibly from the disintegrating neckcanal-cells. The nature of this substance can only be inferred from the fact that it has been found possible to divert sperms from their natural goal by introducing a source of sucrose into the liquid submerging the sexual shoots. Thus it has been observed that sperms will turn aside from archegonia and travel towards, and finally enter capillary glass tubes containing a solution of that sugar. The extreme sensitiveness of the sperms to such influences may be judged by the fact that a solution of 0.001 per cent. exercises an attraction for them. These chemotactic responses are particularly interesting as they afford a chemical and mechanical basis for a phenomenon which might otherwise be attributed to spontaneity or purposeful volition.

The fertilised ovum, or oosperm, in the archegonium divides into an upper and a lower hemispherical cell. They each by oblique divisions give rise to an apical pyramidal cell. The lower one produces a small mass of tissue-the foot-which presses into the bottom of the archegonium, and makes firm connection with it; the upper forms a cylindrical mass which grows much more vigorously and pushes the upper part of the archegonium before it. At first the growth and stretching of the archegonium keeps pace with the elongation of the embryo it contains, but later the archegonium is torn by the embryo's continued growth, and the upper part is separated as a cap-the 
calyptra-on the top of the embryo, the base remaining as a sheath-the vagina-round the foot.

Under the calyptra the upper end of the embryo enlarges and forms the sporangium, or theca, supported on the bristle-like stalk or seta. The theca is pear-shaped with a slight obliquity. Its tapered end is continuous with the top of the seta and is bent,

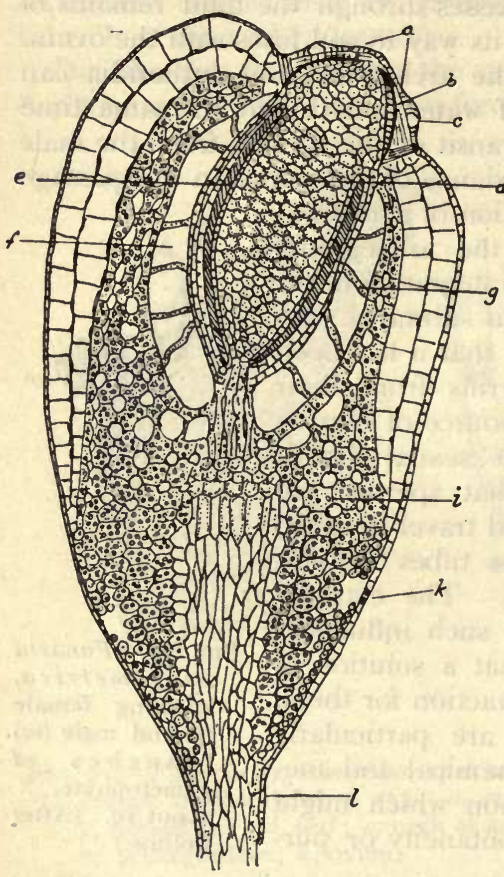

FIG. 29.-Funaria hygrometrica, theca longitudinal section, $\times 40$. $a$, operculum; $b$, peristomium ; $c$, annulus ; $d$, columella ; $e$, sporogenous cells; $f$, air-space ; $g$, suspensory filament ; $i$, photosynthetic cells of apophysis; $k$, stoma; $l$, epiderınis. just where it joins the seta, at right angles. The axial tissue of the seta, which forms a strand of elongated cells throughout its length, is continued up into the tapered base, or apophysis, of the theca. 'This strand has apparently the function of conveying materials absorbed by the foot up the seta to the developing theca.

Externally obliquely pearshaped, the theca may be compared to a thick walled wine-glass contracted at its rim and closed with a small lid-the operculum. The wall of the theca is composed of about five layers of cells. At the bottom, however, it is many layers thick, so that the space enclosed by the wall reaches only about half way down the theca, the lower half being formed by the solid apophysis. In this space is slung a solid cylindrical core. The core is held in position there by elongate cells, passing from it to the inner surface of the wall of the theca, which traverse the thimble-shaped space separating the core from the wall.

In the outer region of the core a layer is differentiated, the cells of which are richer than their neighbours in protoplasm and give rise to the spores. This is the sporogenous layer, and the whole 
mass within this layer forms the columella. 'The upper- end of the columella is cone-shaped and projects into the slightly domed operculum, while its lower end is much attenuated and joins the axial tissue of the apophysis.

Originally the tissue of the operculum is continuous with that of the wall of the theca, but as differentiation proceeds a groove is formed on the outside marking off the operculum and defining the rim of the theca. Immediately above the rim a row of large cells with easily broken radial walls is formed, and its outer walls separate as a continuous band by the rupture of the radial walls. This band is called the annulus. The separation of the annulus leaves the operculum free to fall off. Like the wall of the theca the operculum is a few cells thick. The lower surface of the operculum is cut off from the top of the columella by a single layer of cells, which is continuous with the inner layer of the wall of the theca. The outer and inner walls of this layer of cells are strongly thickened while the radial walls connecting these are thin and early disappear, leaving the thickened walls as two separate membranes over-arching the top of the columella. These two coneshaped membranes split with sixteen radial clefts from the apex to the edge and so form two rows of sixteen teeth each attached to the inside of the rim of the theca. This apparatus is called the peristomium.

As the theca matures, the cells of the sporogenous layer each give rise to four spores; the thin-walled tissue of the columella loses water and shrivels up, tearing open the space in which these spores lie. It is at this moment the cell-walls of the annulus give way and the operculum is cast off, exposing the peristomium. The teeth of this latter are very sensitive to changes in atmospheric moisture and by their hygroscopic curvatures alternately bend in and out of the cavity of the theca, each time raking out a small quantity of the ripe spores, thus scattering them in small quantities over a considerable time. During this period the seta, which is also hygroscopic and twisted, causes the theca to gyrate irregularly. In this way the combined arrangements of the theca form a very efficient mechanism for distributing the spores and preventing them from falling in large masses which would lead to waste by the overcrowding of the developing moss plants. The dependence of the common species of Funaria on the hygrometric state of the atmosphere for the distribution of its spores is marked by the specific title Funaria hygrometrica.

With the production and shedding of the spores the development of the whole apparatus consisting of the foot, seta and theca 
is completed and its function discharged. It then turns brown and withers. Taking its origin from the gametes of the leafy moss-plant it has developed into an organism wholly unlike its parent. It is evidently the asexual spore-producing phase-the sporophyte-succeeding the sexual phase - the gametophyte-and we have to do with an alternation of generations quite similar to that occurring in Marchantia and probably comparable to that found in Polysiphonia. The two phases are relatively simply organised and equally developed in Polysiphonia, while in Marchantia the sporophyte generation is inconspicuous compared to the large green complicated thallus of the gametophyte. In Funaria we have the two generations fairly equalised in size and complexity of structure. In Polysiphonia the two generations are independent of each other from the germination of the reproductive body which gives origin to each of them. This is not the case with Marchantia. There the germinating spore gives rise to the physiologically independent holophytic gametophyte. The generation developing from the oosperm obtains all its substance and nutriment from the tissues of the supporting gametophyte. It never makes attachment with the earth to obtain a supply of the nitrates and other mineral salts absorbed by holophytic plants; it possesses no photosynthetic organs or tissues, whereby it might manufacture carbohydrates from carbon dioxide and water. It develops wholly parasitically on the gametophyte, at first wholly enclosed in it, and only breaking forth from its surface when development is complete and it becomes necessary to shed the spores.

In Funaria, during the early stages of development, the sporophyte is entirely parasitic upon the gametophyte. Through the foot it absorbs all its requirements from the tissues at the base of the archegonium, organic substances; mineral solutions and water. As it develops, chloroplasts are formed in its superficial cells and no doubt supplement the organic substances transmitted from the gametophyte, by carbohydrates produced in situ by photosynthesis. Tissue specially differentiated for this function is developed round the conducting strand in the base of the theca and forms the apophysis.

The cells forming the outer layers of the apophysis are thin walled and contain large numbers of chloroplasts, and the carbon dioxide of the air is conveyed to their surfaces by intercellular passages which extend inwards among the cells from the surface. Lest these intercellular passages should, by permitting too free an escape of water vapour, allow the drying up of the photosynthetic 
cells, their entrance is constricted to a narrow pore or stoma. On each side of the stoma is a curved cell, a guard-cell, reminding one of the similar apparatus round the pore leading into the photosynthetic chambers of Marchantia. But it must be remembered that those pores are structures in the gametophyte, while the stomata of Funaria belong to the sporophyte. It may be remarked here that stomata having the structure of this type are only found on sporophytes, and they occur with very little modification on the sporophytes of all the higher plants. With this photosynthetic organ, the sporophyte of Funaria in its later stages must be almost, or completely, independent of the gameto-

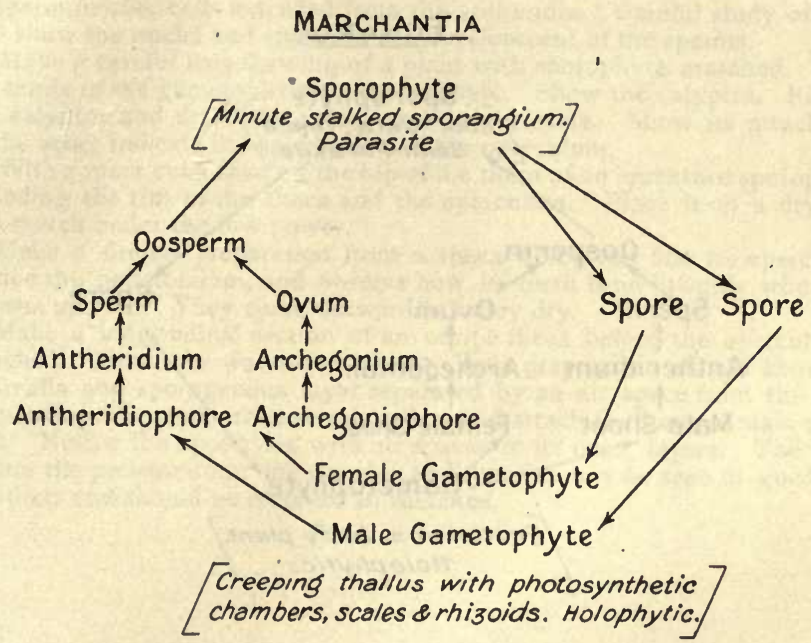

F1G. 30.-Diagram summarising the life-history of Marchantia.

phyte, so far as its carbon supply is concerned. Apparently' however, for all other substances it is dependent on the gametophyte. Hence, its parasitism is not quite so complete as the sporophyte of Marchantia.

The life-histories of Funaria and Marchantia may be summarised by the two schemes shown in Figs. 30 and 31 .

The formation of buds on the protonema and upon the rhizoids is a very prolific method of asexual reproduction. Such reproductive rhizoids are usually formed on the lower parts of the stem but they may arise from almost any part of the gametophyte, especially from the detached parts kept in a moist atmosphere. 
In this way the stem, the leaves and even the archegonium of the gametophyte may, by the production of rhizoids, originate new plants. The sporophyte has the same power, and plants may be derived in like manner from parts of the theca and seta.

An interesting observation has been made on this method of reproduction in Funaria. If leaves from around the antheridia on the male shoot are taken off and induced to give rise to new plants, it is found that these plants can only produce male shoots bearing antheridia. Whereas plants similarly developed from leaves of the female shoot, and even from the archegonium itself, produce the normal bisexual plants with male and female

\section{FUNARIA}

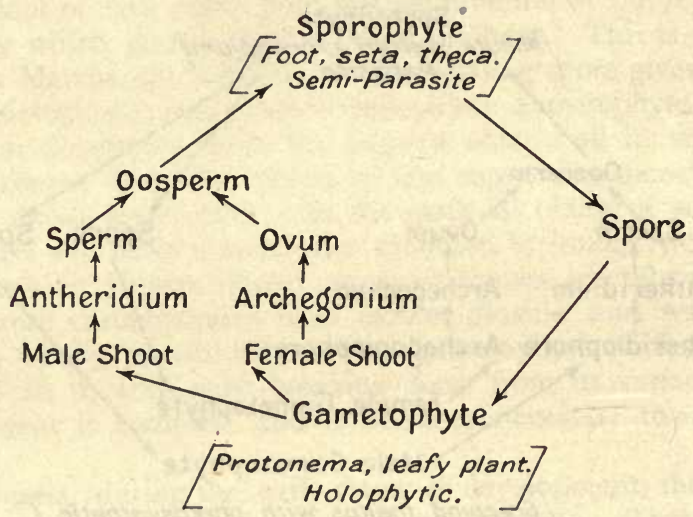

FIG. 31.-Diagram summarising the life-history of Funaria.

shoots. Apparently just before the bifurcation of the stem takes place, a cell division occurs in which the two cells arising receive different potentialities from the parent cell. In this way we may suppose that either a substance is omitted from one that is necessary for the formation of archegonia or something is included which inhibits their development.

\section{PRACTICAL WORK.}

Examine portion of a patch of Funaria and notice how closely the sexual shoots grow together.

Remove a small group of plants by breaking the sod carefully. Wash 
away the sandy soil and expose the rhizoids; lay out a few plants on a piece of white paper, or better, opal glass, and examine with a lens. Make a sketch showing the stem with its branches, the rhizoids and leaves. Also draw a specimen with sporophyte attached. Show the calyptra.

Mount a few leaves in water, sketch with the low power. Also sketch a small group of cells from the blade of the leaf. Record the shape of the chloroplasts. Observe that they are usually ovoid. Sometimes one is seen constricted in the middle. Instances will be found where the constriction is deep and ultimately leads to fission. The chloroplasts multiply by fission. Application of liquor iodi will bring out minute starch grains in the chloroplasts.

Prepare longitudinal sections of the tips of male and female shoots. Make drawings illustrating the form of the antheridia and archegonia. Estimate their size by means of the Ghost-micrometer. Observe the masses of sperm-mother-cells extruded from the antheridia. Careful study of these will show the nuclei and stages in the development of the sperms.

Make a careful lens drawing of a plant with sporophyte attached. Mark the limits of the gametophyte and sporophyte. Show the calyptra. Remove the calyptra and draw the theca on a larger scale. Show its attachment to the seta; indicate the apophysis and the operculum.

With a razor cut a slice off the top of the theca of an immature sporophyte, including the rim of the theca and the operculum. Place it on a dry slide and sketch under the low power.

Make a similar preparation from a theca which has lost its operculum. Notice the peristomium, and observe how its teeth bend inwards when you breathe upon it. They curve outwards as they dry.

Make a longitudinal section of an unripe theca before the operculum is detached. Mount in water and cover. Your preparation should show the columella and sporogenous layer separated by an air space from the wall. Suspending filaments traverse this space and attach the central mass to the wall. Notice the apophysis with air spaces in its outer layers. The operculum, the peristomium, the annulus and the rim may be seen in good preparations and should be recorded in sketches. 


\section{LECTURE XIX.}

ThE "Male Fern" Aspidium filix-mas is easily procured for study. It is one of the commonest of our native ferns and is found in large quantities in hedgerows and woods. Most people are familiar with its large, green, graceful leaves, converging to a centre where are found the young, incurved leaves, covered with golden-brown scales, reminding one of the furry paws of an animal. The mature leaves together form a funnel-shaped crown, 2 or 3 feet in diameter. At the margin of the funnel are the recurved leaf-tips. Their stalks run down together to the top of the stem. The stalk of each leaf is continued upwards as a central shaft-the rachis. On each side of this there is a series of leaflets-the pinnægradually diminishing in size from below upwards. Each pinna is attached to the rachis by a short stalk which is continued outwards as a mid-rib in the leaflet. The general outline of each pinna is feather-shaped, its length being about ten times its breadth at the base, but its margin is deeply indented. The indentations are often so deep that they reach the mid-rib, and may divide the pinna into secondary leaflets-the pinnules. This is especially the case in the inner parts of the basal pinnæ. The lower parts of the rachis and of the leaf-stalks are covered more or less thickly with golden-brown scales.

The stem can only be satisfactorily seen by digging up the plant and washing away the soil which adheres to it. It will then be found to be an irregular, rough and often somewhat curved cylindrical mass, entirely covered over with the bases of leaves. The part which is buried deepest in the soil is somewhat narrower than the upper end and converges to a point. The top of the stem is covered over by the incurving young leaves, each of which is curled inwards and rolled up on itself; below and outside these are the fully developed green leaves. The lower part of the stem is closely covered with the quill-like remains of the partially-decayed bases of older leaves. From these older leaf-bases numerous roots come 
off. These are dark brown in colour, cord-like and almost wiry in consistency. The soft tissues and young leaves are at the top and the hard material and old leaves at the base of the stem. Evidently it grows at the top and there forms the new leaves.

The relation of the leaves to the stem is best seen by splitting the latter down longitudinally. The upper part of the stem is composed of whitish, cheese-like and somewhat slimy tissue.

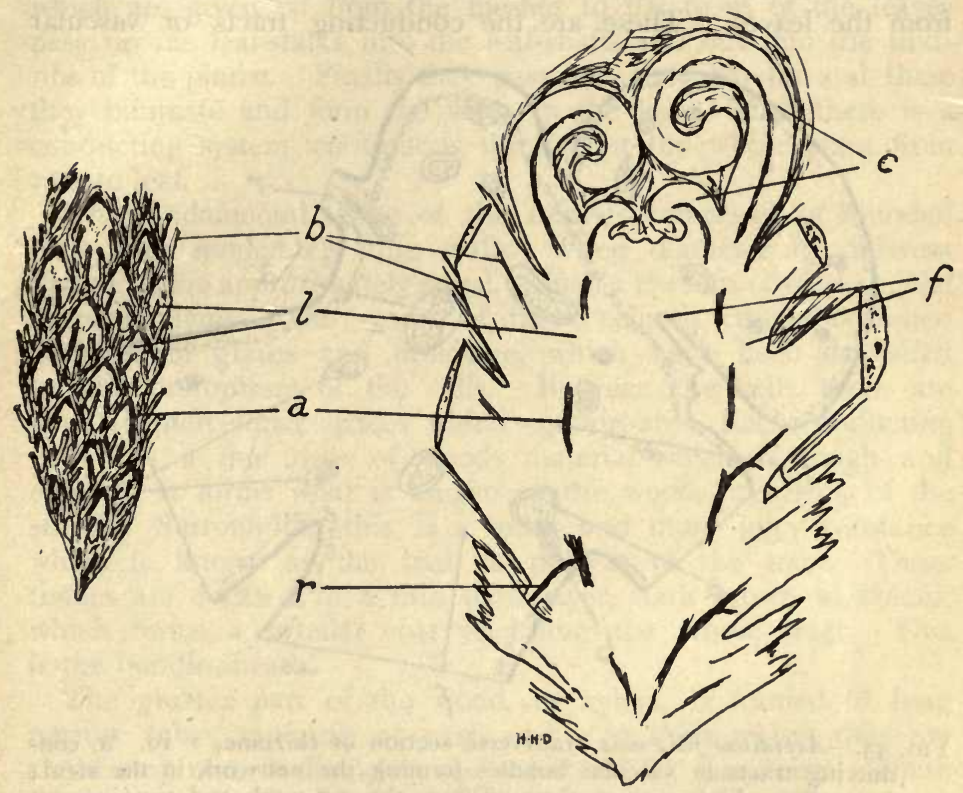

Fig. 32.-Aspidium filix-mas: on the right, stem cut longitudinally, $\times 3$. $a$, net-work of conducting tracts; $b$, branches from net-work supplying leaves; $c$, young leaves curved over apex; $f$, fundamental tissue; $l$, meshes of net-work forming leaf-gaps; $r$, conducting tract of root; on the left is shown the net-work with the fundamental tissue removed.

The extreme top is a low cone. Viewing the cut surface with a lens small prominences on the side of this cone may be made out. These prominences are the beginnings of leaves which in a year's time will have grown into small incurved leaves, similar to those which now are seen outside them curving over the top. In the meantime those which are now in this position will have developed into mature foliage-leaves. The 
foliage-leaves retain their fresh green colour for one season and then wither back, leaving only their base attached to the stem. On the edge of the cut surface of the longitudinally split stem we can make out the attachment of many of these older leaves.

On the cut surface fibrous tracts of tissue are visible here and there. These tracts are cord-like and are found in the stem close to the bases of the leaves. Similar but finer cords enter the stem from the leaves. These are the conducting tracts or vascular

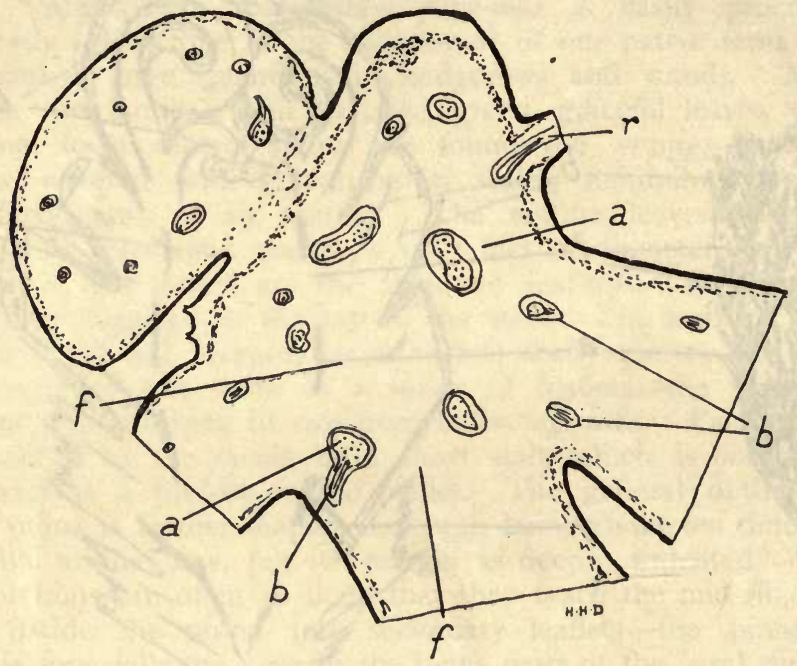

FIG. 33.-Aspidium filix-mas, transverse section of rhizome, $x$ Iо. $a$, conducting tracts or vascular bundles forming the net-work in the stem; $b$, small conducting tract given off from the net-work and running out into the leaves; $f$, fundamental tissue; $r$, conducting tract entering a root.

bundles which transmit water and other materials through the plant. They form a tubular net-work in the stem, close to the bases of the leaves. Each mesh of the net-work which is irregularly rhomboidal in outline corresponds to a leaf base. Threadlike tracts are given off from the inside of the mesh, which, converging slightly towards one another, then turn upwards and pass out into the corresponding leaf. This net-work of conducting tracts is embedded in the soft white slimy substance of the stem which is called the fundamental tissue. The roots also 
contribute cord-like tracts to the net-work of conducting tracts in the stem. There are usually about three roots which emerge from the base of each leaf. Each of these roots has an axial conducting tract which passes from the root into the base of the leaf-stalk just above the junction of the latter with the stem. It then makes its way across the outer fundamental tissue of the stem and joins with a tract of the net-work. The tracts which are given off from the meshes to the bases of the leaves pass up the leaf-stalks into the leaf-shafts and out into the midribs of the pinnæ. Finally they pass into the pinnules and there they bifurcate and form the veins in the leaf. Thus there is a conducting system continuous throughout the whole plant from root to leaf.

The fundamental tissue of the stem is composed of rounded cells with moderately thin walls. Their diameter in different directions are approximately equal. Such a tissue is often described as parenchyma. The cavity of these cells is chiefly occupied with starch grains and mucilage, which have been deposited by the protoplasm of the cells. Between the cells there are angular intercellular spaces which contain air. Each conducting tract has a fine core of woody material which is tough and elastic. It forms what is known as the wood, or xylem, of the strand. Surrounding this is a softer and more juicy substance which is known as the bast or phloem of the tract. These tissues are encased in a thin hard layer, dark brown in colour, which forms a tubular coat enclosing the whole tract. This is the bundle-sheath.

The greater part of the wood, or xylem, is formed of long narrow tubes tapering at each end. At their widest they are about $0.05 \mathrm{~mm}$. in diameter, and are about $\mathbf{I}-5 \mathrm{~mm}$. long. These tubes are called tracheids. In transverse section they appear polygonal. The walls of these tubes are formed of wood which is a substance derived from cellulose, differing from it in its physical and chemical properties. It is more rigid, more permeable to water and less ductile than cellulose. The walls are of considerable thickness but have thin places upon them called pits. The pits of the tracheids of the ferns usually have the form of transverse .slits which are quite close together, so that the thick portions between them may be compared to the rungs of a ladder. Hence these tubes are called scalariform tracheids. The tapering ends of the tracheids overlap each other in the xylem core and where they are in contact with one another the pits on the adjacent walls correspond, so that at these points the 
dividing partition between two tracheids is extremely thin. Among these scalariform tracheids there are one or more strands of tracheids having thin walls supported by an internal spiral thickening. These tubes are narrower than the scalariform tracheids and being formed first are called protoxylem. The cavities of the tracheids contains only water or dilute solutions. There is no protoplasm or granular contents.

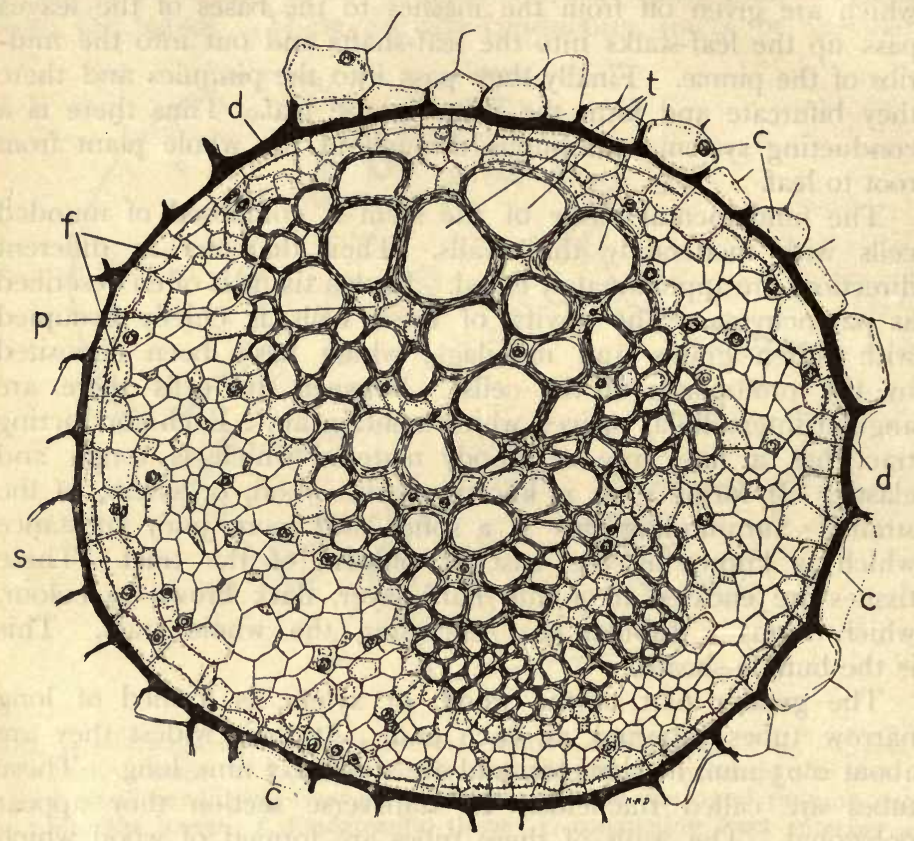

FIG. 34.-Aspidium filix-mas, small conducting tract or vascular bundle, transverse section, $\times 250$. $c$, inner walls of cortex forming bundle sheath ; $d$, endodermis; $f$, pericycle ; $p$, wood-parenchyma; $s$, sievetubes in bast; $t$, tracheæ in wood.

The phloem, which is the soft layer covering the xylem strand, is chiefly composed of sieve-tubes. Like the tracheids they too are spindle shaped and polygonal in cross section. Their walls are very thin and are formed of cellulose, and where two sieve-tubes adjoin are marked out into irregular areas-the sieve-plates, which are perforated by exceedingly fine pores. The tubes are lined with a very thin film of protoplasm which is continuous with 
the protoplasm of adjoining sieve-tubes through the perforations of the sieve-plates. The central cavity of the sieve-tubes is filled with a dilute sol of proteins often containing also dissolved sugar. Filling up the interstices between the tracheids in the wood and the sieve-tubes in the bast are thin walled polyhedral cells with cellulose walls and protoplasmic, and often starchy contents. Their diameters in different directions being approximately equal, they are called respectively the wood and bast-parenchyma. The outer-

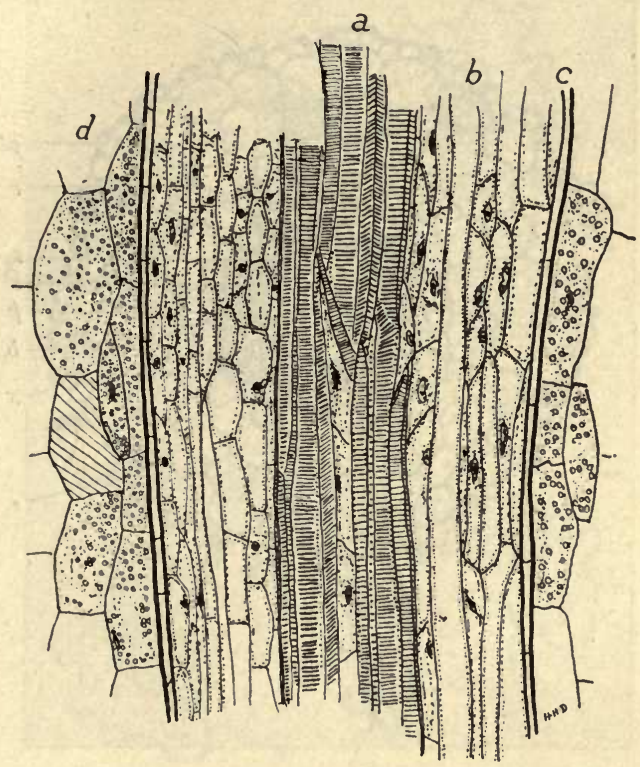

FIg. 35.-Aspidium filix-mas, small conducting tract or vascular bundle, longitudinal section, $\times$ r7o. $a$, tracheæ; $b$, bast ; $c$, bundle sheath.

most layer of the conducting tract is formed by the endodermis. It is easily recognised by its being next the brown cell-walls of the sheath isolating the strand from the surrounding fundamental tissue. The cells of the endodermis are oblong. Their greatest length is parallel to the axis of the bundle and they fit together closely, leaving no gaps. Between the endodermis and the outside of the bast is a single layer of cells, the pericycle. The cells of the pericycle roughly correspond with those of the endodermis. The walls of the endodermis and pericycle are uncoloured. 
The outer layers of the fundamental tissue of the stem have very thick, hard and brown cell-walls, forming a tissue known as sclerenchyma. It forms a continuous coating over the bases of the mature leaves and the stem, and is only penetrated by the conducting strands entering from the roots.

The roots have a comparatively simple structure. A single conducting tract runs down the axis of each. It is covered by a

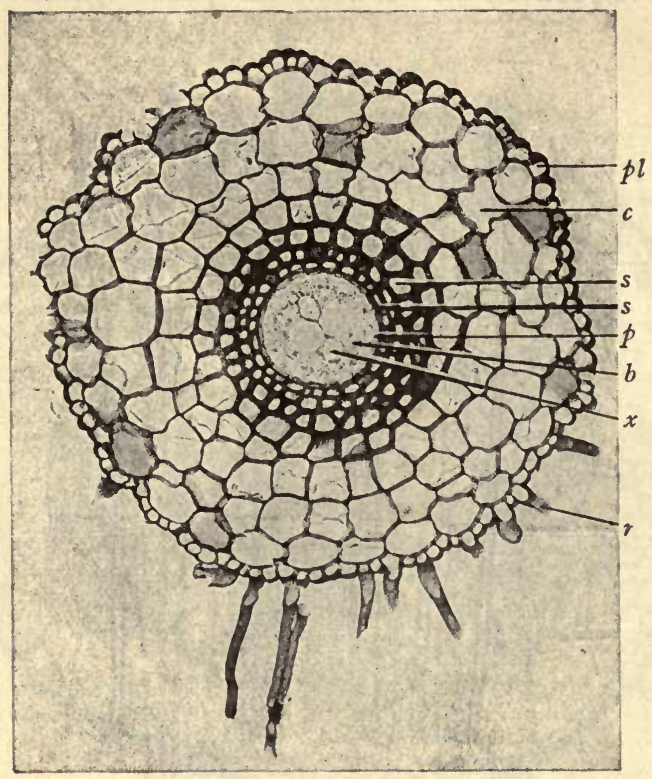

FIG. 36.-Aspidium filix-mas, root, transverse section, $\times 350$. $b$, bast; $c$, cortex; $p$, pericycle; $p l$, piliferous layer; $r$, root-hair $s$, sheath of conducting tract; $x$, wood. (From Evans' An Intermediate Textbook of Botany.)

thick coat of fundamental tissue - the cortex. In the outermost layer of the cortex certain isolated cells grow out at right angles to the surface and develop into root-hairs which grow out among the particles of the soil and absorb water and various dissolved substances. The inner layers of the cortex develop thick and brown walls and form a sclerenchymatous sheath round the conducting tract. An apical growing cell, like that of Funaria, adds to the root at its tip. It is pyramidal in shape. Its apex points towards 
the base or attachment of the root. From the sides of this cell are formed flat segments which produce the cortex and conducting tract. Its base forms segments which give origin to an irregular cap-like mass, the outer cells of which have little cohesion and simply form a lubricating substance to facilitate the forward passage of the growing root through the soil.

The leaves are essentially organs for exposing the photosyn-

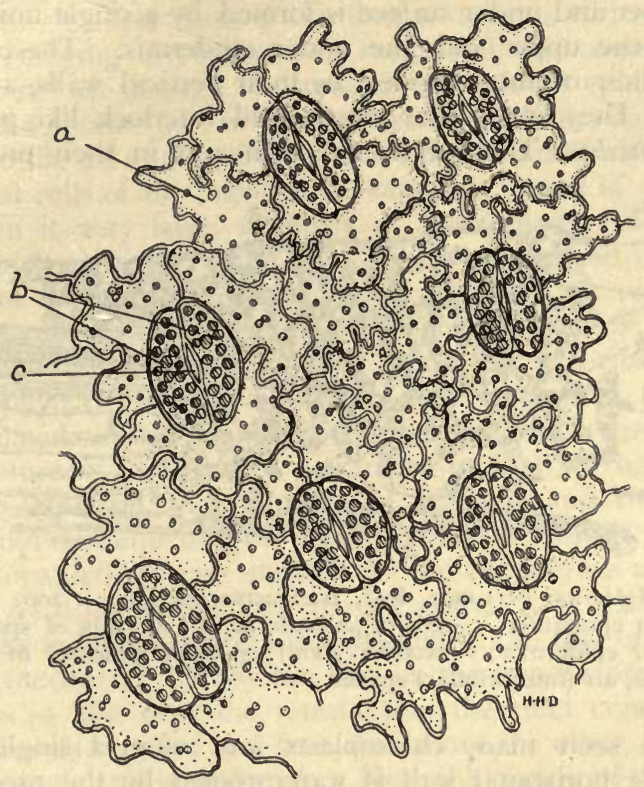

FIG. 37.-Aspidium filix-mas, lower epidermis, $\times$ 300. $a$, epidermal cell with scattered chloroplasts; $b$, guard-cells with crowded chloroplasts ; $c$, stoma.

thetic cells to the light and air. The leaf-stalk and rachis have the function of supporting these cells in a suitable position and maintaining their communications with the rest of the plant. The leaf-stalk is a rigid tube formed of the outer sclerenchymatous layers of fundamental tissue continuous with that of the stem. This tube is filled with parenchymatous fundamental tissue continuous with the soft fundamental tissue of the stem, but differing from it in that its cells contain chloroplasts. Embedded in this 
soft green fundamental tissue are the thread-like conducting tracts given off from the meshes of the network in the stem. A similar arrangement of tissues is found in the mid-ribs of the pinnæ and pinnules. In the upper parts of the rachis the sclerenchymatous walls are reduced and finally disappear. The photo-synthetic tissue forms a wing on each side of the mid-ribs, in which the ultimate branches of the conducting tracts bifurcate.

The green tissue of the pinnules is about $0.75 \mathrm{~mm}$. thick. Their upper and under surface is formed by a single uniform layer of cells-the upper and the under epidermis. These cells are flat and their outlines, formed by their vertical walls, are sinuous or wavy. They fit together closely and interlock like pieces of a jig-saw puzzle. Their walls are thin and in their protoplasmic

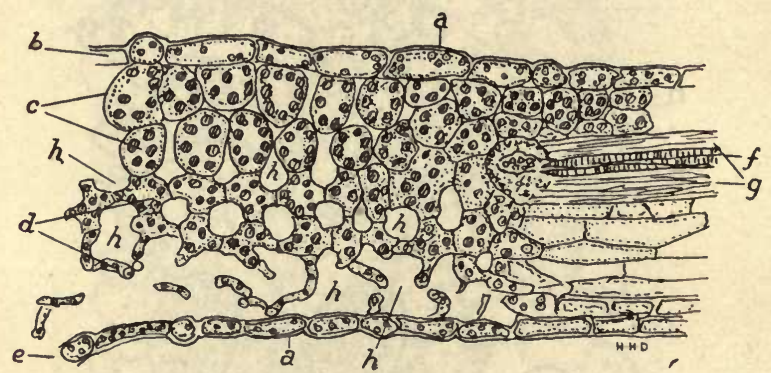

FIG. 38.-Aspidium filix-mas, leaf, transverse section, $\times 200$. a, cuticle; $b$, upper epidermis; $c$, cells of palisade layer ; $d$, cells of spongy layer ; $e$, lower epidermis; $f$, wood (xylem); $g$, bast (phloem) of conducting tract; $h$, air (intercellular) spaces.

lining are seen many chloroplasts and often a single nucleus. Their outer horizontal wall is waterproofed by the presence of a substance called cutin in the cellulose. The outer walls of the adjacent cells form a continuous membrane over the whole leaf, called its cuticle. The cells of the upper epidermis fit together without leaving gaps. In the under epidermis, however, minute slits are found - the stomata. They are about $0.02 \mathrm{~mm}$. in length and may open about $0.004 \mathrm{~mm}$. wide. There are about thirty or fifty of these slits to the square millimetre. The stomata are limited on each side by a guard-cell. 'The guard-cells are crescentic and bend round the stomata. They are much richer in chloroplasts than the other epidermal cells.

Between the upper and lower epidermis is the mesophyll-the photosynthetic tissue proper of the leaf. It is composed of five or 
six layers of thin-walled cells of irregular shapes. Near the upper epidermis the mesophyll cells fit more closely together, while in the neighbourhood of the lower epidermis they are very loosely put together and leave between them large spaces. There is no sharp division between these two layers in this leaf as there is in many others; but notwithstanding this, they may be called the palisade and spongy parenchyma respectively. The spaces between the cells of the spongy parenchyma and the smaller ones between those of the palisade layer form a continuous system of irregular airpassages ramifying throughout the mesophyll, and communicating with the outer air through the stomata in the lower epidermis. In this way the greater part of the surface of each mesophyll cell is exposed to the air and is free to interchange gases with it. The thin-walled cells of the mesophyll contain a lining of protoplasm carrying in it very large numbers of chloroplasts. The central space of each cell is occupied by a large vacuole; and the osmotic pressure of its dissolved substances bearing on the protoplasmic lining distends the cell-walls and keeps the cells stiff or turgid.

The conducting tracts emerging from the mid-ribs penetrate the mesophyll. They travel in the upper region of the spongy parenchyma about half-way between the upper and lower epidermis. The structure of the tracts is similar to that we have already studied in the stem. The number of tracheids and sieve-tubes is reduced and consequently the tract becomes finer. It is throughout accompanied by and encased in the endodermis and bundle sheath. As it passes out into the mesophyll it is still further reduced - the reduction being most marked in the bast on the upper aspect of the tract. Finally the bast on the upper side completely disappears so that near the termination the tract consists of two parallel strands of wood and bast, one above the other, surrounded by the pericycle and endodermis. Near the margin of the pinnules each of these attenuated tracts bifurcates.

Outside the endodermis the cells of the mesophyll are elongated and accompany the tract as an ill-defined sheath. These cells are poor in chloroplasts and with the conducting tracts constitute the veins of the leaf. Fitting closely together they cut off the strand from the intercellular system of the mesophyll.

Enough of the structure of Aspidium has already been described to show that this fern is a very complicated and highly developed organism. Its organs are highly specialised to perform various functions and these functions are broadly conditioned by the fact that it is a holophyte which lives upon land. The materials needed for growth are obtained partly by the root-hairs, 
water, nitrates and other mineral substances from the soil, and partly by the mesophyll, carbon dioxide from the air. The wide separation of these parts necessitates a very perfect system of transport. This is provided in the conducting tracts or vascular bundles. The motive force to effect this transport is the heat energy absorbed by the leaf-cells. Energy absorbed by the mesophyll cells, either in the form of light or heat, causes evaporation from their surfaces into the air-spaces of the leaf. This loss tends to diminish the bulk of the watery contents of the vacuoles of the cells. But the osmotic pressure of the dissolved substances in the vacuoles opposes the collapse of the protoplasm and presses it outwards, so water has to be drawn into the vacuole. A supply for this is at hand in the cells of the sheath which in turn draw water from the woody tracheids. These latter afford an easy passage for water rising from the roots through the stem and leaves. Thus the evaporation from the mesophyll cells draws the water up through the capillary tubes of the wood of the conducting tracts from the root, where the supply is kept up by the absorption of the root-hairs.

Evidently in a low-growing plant like the fern if evaporation removes water from the upper end of the tracheidal tubes the atmospheric pressure acting on the water surfaces round the root-hairs would force up water to take the place of that which is lost by evaporation. But in plants much higher than $30 \mathrm{feet}$, which is near the limit to which atmospheric pressure can raise water, evidently such a mechanism must break down. As a matter of fact even low-growing plants need not rely on atmospheric pressure for the lifting of the water in their tracheidal tubes. For water, when contained in vessels which it thoroughly wets (as in the tracheids) and completely fills, leaving no bubbles, transmits a pull just in the same way as a metal wire would. Thus the removal of water from the mesophyll cells by evaporation produces a pull in the water of the vacuoles; this is transmitted to, and down through, the water contained in the tracheidal tubes throughout the conducting tracts of the plant. The mesophyll cells are prevented from collapsing under this stress by the osmotic pressure of the dissolved substances in their vacuoles, while the ladder-like and spiral thickenings of tracheids support their thin walls and prevent them buckling inwards. Evidently the pits are essential in order that the flow of water may be facilitated from one tracheid to another.

The elimination of water vapour from the mesophyll cells through the passages and stomata is called transpiration and the 
current which it produces in the tracheids is called the transpiration-current. It is evidently of great importance in bringing a supply of water and dissolved mineral substances to the mesophyll cells. The water thus supplied enables these cells, containing chloroplasts, surrounded with air and exposed to light, to manufacture carbohydrates. In the dissolved substances carried by the transpiration-current these cells also find the remaining elements needed for the building of protoplasm, and it is highly probable that the mesophyll cells are the laboratories in which not only carbohydrates but also proteids and protoplasm are synthesised.

After the leaf has attained its definite size, the indefinite retention and accumulation of these substances in it would serve no purpose. They are required, however, at the growing regions of the stem and root where new cells are being formed and tissues differentiated. 'The transport of these materials to the growing' regions is usually assigned to the bast, or phloem, of the conducting tracts and more particularly to the sieve-tubes. The mechanism of their transport is, however, unknown. Their presence in the sieve-tubes may be detected; and by breaking the continuity of the bast, growth at the tips is suspended, but further than this little is known of the process.

The solution absorbed from the soil is very dilute and to obtain sufficient quantities of the necessary materials transpiration and evaporation are evidently of importance. These processes have also another essential rôle. For the purpose of photosynthesis leaves must be displayed to the light and are often unavoidably exposed to direct sunlight. The light absorbed being largely converted into heat would lead to a rise of temperature beyond the limits of resistance of the cells, were it not that evaporation cools the leaf. This function may be readily appreciated by exposing side by side in the sun a living leaf with a piece of dry but equally light-absorbing material-or a dead dry leaf. The living leaf will remain cool to the touch while the others become hot.

When the leaves of the fern are exposed to light, the process of photosynthesis liberates oxygen among the green cells of the plant, and part of this oxygen is used in respiration by those cells. In darkness when oxygen is not produced within the green tissues and always in the colourless tissues of the stem and root, oxygen must be conveyed to the cells. For it is easy to show that Aspidium cannot continue to respire anaerobically without injury. This supply of oxygen finds its way to the cells throughout the plant by means of the air-passages and intercellular spaces in the 
tissues. The cells which are not in direct contact with these passages or with the outer air, must obtain their oxygen supply in solution from adjacent cells. The sluggish life of the plant which is practically without movement, and does so very little external work, explains how it is that gaseous and liquid diffusion through these channels is sufficient to keep up the requisite supply.

Life on the land necessitates mechanical properties different from those which are suitable to aquatic organisms. These properties are chiefly associated with the supporting or skeletal tissues. In water, buoyancy associated with a certain amount of tenacity is sufficient to attain the exposure of the photosynthetic organs to light, and to secure them against injury. Sub-aerial plants must support their leaves on rigid structures to display them to the light, but they must yield in wind and recover their position when favourable conditions return. The requisite rigidity is obtained by a suitable disposition of sclerenchyma and the elastic recovery is usually provided for by a combination of parenchyma with sclerenchyma or by means of parenchyma alone. Thus the bases of the leaf-stalks which must always stand erect are constructed out of a thick-walled tube of sclerenchyma forming their superficial tissue. The cells of this sclerenchyma have thick hard walls and they are not easily stretched or compressed so that the lower part of the stalk is very rigid. To displace it at all one side must elongate and the other contract. If, however, any distortion is produced, evidently this tube must be flattened and its volume correspondingly reduced. Such a reduction is not only resisted by the walls of the tube but is opposed also by the cells of the fundamental tissue within it. Any pressure on them tends to stretch their walls, for their vacuoles are practically incompressible, and hence a tension on every cell-wall throughout the tissue acts to restore the original position of the leaf-stalk. In the upper parts of the rachis where there is less sclerenchyma and more thin walled tissue, the rigidity of the organ diminishes but its flexibility increases. Here the flexure of the rachis is chiefly resisted by the walls of the parenchyma. The bending produces a pressure on the cells which distorts them more and more from the spherical form. Owing to the incompressibility of the vacuole their volume must remain the same, therefore their wall is stretched. When the bending force is removed the pressure disappears and the elasticity of the cellulose walls restores the cells to their more spherical original shape. In this region the sclerenchyma-or mechanical tissue as it is called-is not continuous round the rachis and consequently only acts like 
isolated bands, those on the convex side alone taking up the tension. Those on the concave side being very slender are only to a very small degree mechanically efficient.

The pinnæ and pinnules practically owe their entire mechanical support to the osmotic pressure of the substances in their vacuoles acting as a compression member and to the tenacity of their cellwalls and to those of the conducting strands acting as a tension member. The destruction of one of these members, as in an engineer's girder, leads to the collapse of the structure. This may be seen by destroying the semi-permeability of the protoplasmic membranes in the cells and thus rendering the osmotic pressure ineffective. The easiest way of doing this is to kill the protoplasm of the leaf either by surrounding it with steam or a poisonous vapour. The pinnæ immediately hang down in a flabby condition.

These considerations show that the usual comparison of the veins of the leaf to the ribs of an umbrella and of the soft tissue to the silk is mechanically unsound. The cell-walls of the veins and soft tissue being the tension members of the system are in reality mechanically equivalent to the silk of the umbralla, while the osmotic pressure of the vacuoles acting on the protoplasmic lining of the mesophyll cells is the compression member and corresponds to the ribs of the umbrella.

\section{PRACTICAL WORK.}

Dig up a plant of Aspidium, shake it free from the adhering earth, and sketch it about one-fifth natural size, showing the large leaves, the incurved young leaves and the rough remains of the old leaves covering the stem. Show some of the roots and their attachment and the tapering oldest part of the stem.

Pull off one or two of the older leaf-bases and note that each carries with it two or three dark brown roots which emerge from the very base of the leaves. Also notice that the conducting tracts usually break across at some little distance up in the leaf-stalk and when the leaf is removed remain attached to the stem. 'They appear as short threads hanging out of the fractured surface.

Cut off the leaves and leaf bases as close as possible to the stem. Sketch one side of the stem showing the cut surfaces of the leaf-stalks, each showing five or six conducting tracts cut across as they pass into the stem. The bases of the leaves are separated from one another by very dark brown grooves. These grooves are lined by the superficial sclerenchyma of the stem and this is the only superficial tissue of the stem exposed.

Cut the stem longitudinally down its median axis with a strong sharp knife. Put one half to steep in ro per cent. nitric acid. Smooth the cut surface of the other half with a razor. Sketch this surface about half its actual size. Note the flattened apex, the incurving young leaves and leaf bases of the older ones. Show the dark brown sclerenchyma forming a tubular casing for the leaf bases and the whitish fundamental tissue of the stem and leaf-stalks. Mark the large portions of the conducting tracts exposed in the stem and the finer tracts given off from these and passing outwards and upwards into the fundamental tissue of the leaf stalks. 
Take the half of the stem which has been soaking in the dilute nitric acid, wash it and with the head of a large pin carefully scrape away the axial column of fundamental tissue and expose the net-work of large conducting tracts from the inside. These will appear pink, while the fundamental tissue is coloured deep orange by the acid. Where the section runs longitudinally in a leafbase the tracts passing down it may be displayed by scraping away the fundamental tissue and even their connection with the sides of the mesh corresponding with the leaf-base may be ascertained. A sketch should be made of the net-work of conducting tracts and of its branches.

Cut a transverse section of a leaf-stalk. Mount in water and cover. If permanent preparations are desired the sections, from fresh material, should be soaked for some minutes in spirit, and then washed in water. If material preserved in spirit is used the sections may be placed in water immediately; select out the thinnest of the sections-and they must be very thin-and place them in a staining-glass containing a mixture of watery saffranine and Delafield's hæmatoxylin. When they are sufficiently deeply stained, i.e. in ten minutes to half an hour, wash in water and pass through two changes of spirit to anhydrous spirit which must be closely covered. Anhydrous spirit may be prepared by putting roasted copper sulphate into ordinary spirit. The anhydrous copper sulphate dehydrates the spirit in contact with it. The volume of the roasted copper sulphate should be at least about quarter that of the spirit treated. In this way most samples of commercial spirit may be rendered sufficiently anhydrous. Of course the spirit, during treatment and till use, should be kept in a tightly-stoppered bottle. From the anhydrous spirit the sections should be transferred to oil of cloves. In this at first it is necessary to keep the sections forcibly submerged. Otherwise they come to the surface and absorb water from the atmosphere. When the sections have become quite clear the surplus oil should be removed from them by touching them with absorbent paper. They may then be placed in a drop of Canada balsam on a slide and covered.

The general outline of the leaf-stalk is convex on the lower side and flat or even slightly concave on the other. The greater part of the section is composed of fundamental tissue. The outer layers are thick walled and sclerenchymatous, and form the sclerenchymatous tubular casing of the leafstalk. In the unstained section these walls are colourless; they take a red colour from the staining mixture. The walls of the inner fundamental tissue are much thinner. Their outline is circular in the transverse section. They take a purple colour from the stain. Embedded in the thin-walled fundamental tissue are the cross sections of several conducting tracts. They are circular or oval in outline. The wood-tracheids have thick walls which stain red, the bast.walls are thin and stain purple, while the bundle-sheath has walls which are orange-brown in colour. A drawing of one conducting tract should be made under the high power.

Next prepare a transverse section of a pinnule. This may be most easily done by rolling several pinnules in a tight cylinder in order to give mutual support. Sections of this cylinder may now be cut with a razor and floated out in water. A large number should be prepared and the thinnest selected mounted in boiled water and covered for examination. When a suitably thin section is found it should be sketched and the drawing should show the upper epidermis, the palisade and spongy parenchyma and the lower epidermis. Also the position of the conducting tracts should be marked.

Portions of the upper and lower epidermis should be sliced off other pinnules and mounted. These will show the peculiarly shaped epidermal cells and the stomata. Estimate the size of the stomatal openings with the Ghost-micrometer. 


\section{LECTURE XX.}

So far we have considered the outstanding external features and the most important points in the gross and minute anatomy of Aspidium, and we have seen in a general way how the construction and the arrangement of its parts meet the requirements of the plant for nutrition, respiration, and growth. It now remains to examine the outlines of its life-history and its methods of reproduction.

During the summer leaves are unfolded which, while they resemble in shape and structure the foliage-leaves formed early in the year, present this difference that the upper pinnæ are studded over on the under surface with numbers of round, brown patches, about a couple of millimetres in diameter. Examined with a lens each patch is seen to be composed of a heap of rounded bodies. Each heap is called a sorus. There are some five or six sori on each pinnule. The sorus is covered over by a membranous scale, the indusium, which is circular in outline with a deep indentation on one side. The indusium is held in position by a short stalk or column attached to the under surface of the leaf and rising up from the midst of the sorus. The bodies forming the sorus are sporangia. Each has the form of a biconvex lens and it is attached

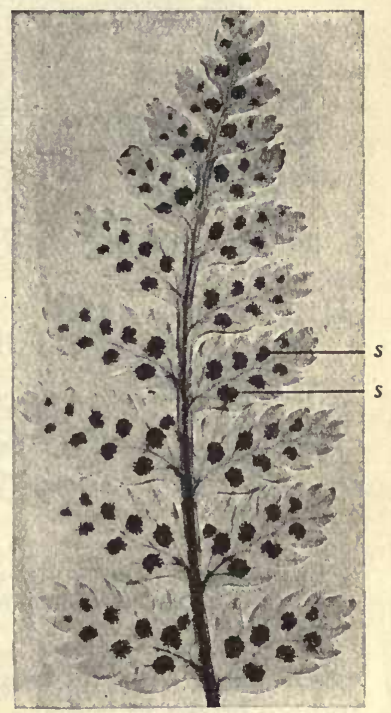

FIG. 39.-Aspidium filix-mas, pinna of leaf. $s$, sorus. (From Evans' An Intermediate Textbook of Botany.)

to the leaf by a slender stalk. The diameter of a sporangium is about $0.2 \mathrm{~mm}$., and the length of its stalk is somewhat more than its diameter. The sori are found on the surface of the leaf immediately below a conducting tract. Here the tissue of the leaf 
forms a little rounded cushion, the placenta, and to this the stalks of the sporangia and of the indusium are fixed. The structure of the sporangia is peculiar and most interesting, as revealing a beautiful natural mechanism for the distribution of the spores. Each sporangium is attached by its edge to its stalk. A single row of cells forms a ridge round its edge. This row is composed of two unequal parts. The longer part, the annulus, which occupies three-quarters of the circumference starting from the attachment of the stalk, is composed of about fifteen similar cells. Each is approximately cubic in form. Its inner wall and those

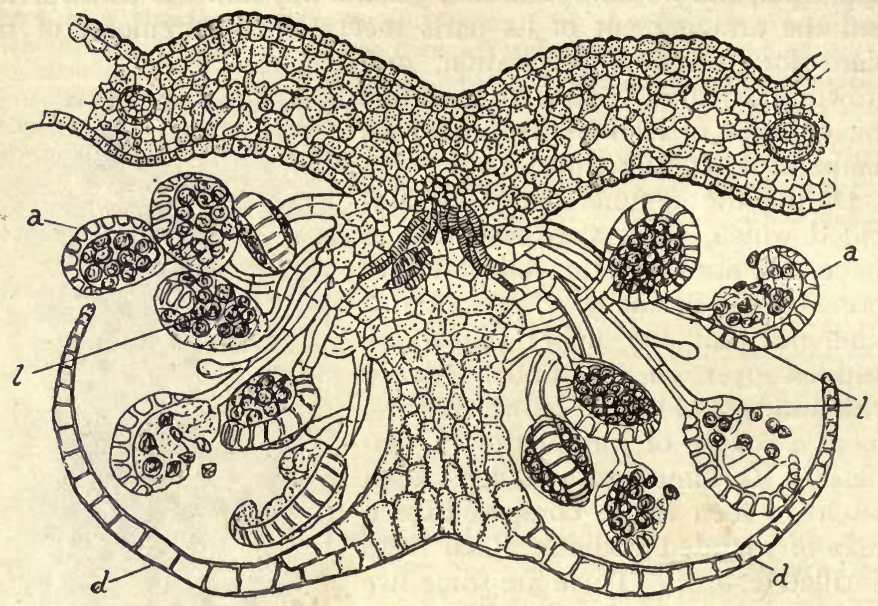

FIG. 40.-Aspidium filix-mas, sorus on leaf, transverse section, $\times 45$. $a$, annulus ; $d$, indusium ; $l$, stomium or lip-cells. (After Kny.)

separating it from its neighbours in the row are much thickened and are brown in colour. The material of these walls is very rigid and elastic and forms a U-shaped band partly enclosing each cell, while that of the remaining outer and side walls is soft and pliant like pure cellulose. The cells of the annulus soon lose their protoplasmic contents but remain filled with water. The remainder of the marginal row, the stomium, is composed of uniformly thinwalled cells, more flattened in form than those of the annulus. Their thin walls are easily torn asunder. The convex sides of the sporangium are formed of a single layer of flat cells, also with thin walls. The cavity enclosed by the annulus and sides is filled with 
forty-eight small brown spores. In their early stages they adhere together in fours, showing that they have been developed as tetrads in separate mother-cells. As the spores and the sporangium ripen, the water filling the cavity of the cells of the annulus diminishes in volume by evaporation through the outer thin walls. The cohesion of the water and its adhesion to the walls draws the walls together. The thin outer and side walls collapse under this tension and are thrown into folds, while the U-shaped radial and inner walls, resisting the pull more effectively owing to their rigidity, are bent into a $\mathrm{C}$-shape. This alteration of form of the component cells leads to a shortening of the outer margin of the annulus and changes it from being convex to being concave externally.

This distortion of the annulus tears the cells of the stomium asunder and makes a rent in the sporangium which spreads radially inwards across the wall. Thus the lower half of the sporangium as it hangs from the under side of the leaf with the spores lying in it; is bent backwards by the contraction of the annulus, the rigid walls of each cell of the latter being compressed like a C-shaped spring by the tension in the water. As evaporation proceeds the tension mounts higher and higher till at last the cohesion of the water is overcome and the water breaks. The tension thus relieved releases the spring, which immediately recovers its original U-shaped form, and in doing so jerks out the spores in the sling-like portion of the sporangium attached to the annulus. The tension at which the break occurs has been found to be about three hundred atmospheres. If the break does not occur simultaneously in all the cells of the annulus, the breaking of the water successively in different cells communicates a series of jerks to the sling, and the spores from each sporangium are ejected in small numbers at a time. The efficiency of this mechanism for distributing the spores at different intervals and in different directions will be realised when it is remembered that the sporangia in the sori ripen successively and that they are oriented in all directions round the placenta.

The spores when ejected are tetrahedral in form with a brown cuticularised outer layer to their cell-wall. They contain a nucleated mass of protoplasm, including many chloroplasts. Once on damp soil they absorb water and become spherical. The inner layer of wall is forced out and breaks through the cuticularised layer, thus forming a tubular outgrowth into which the cell-contents pass. The chloroplasts elongate, divide, and multiply. The tubular cell divides and a filament is formed. Sooner or later 
colourless outgrowths are formed from some of the cells of the filament. These outgrowths possess no chloroplasts and they insert themselves between the particles of soil. They are rhizoids. The cells of the filament enlarge and divide. The divisions in the cells of the filament, which were at first only transverse, are now often longitudinal and so the filament is gradually converted into a plate of cells, and at the same time cell division becomes more and more limited to a single cell on the margin and to its immediate segments. This cell occupies a depression in the margin and the growth of its segments confers slowly a roughlytriangular form on the plant. The apex being occupied by the

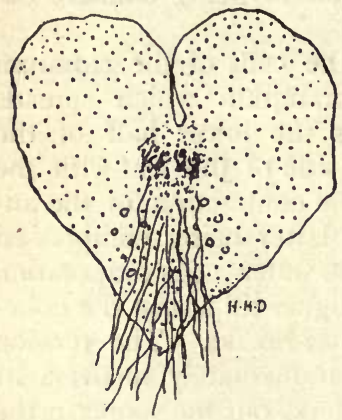

FIG. 4r.-Aspidium filix-mas, under-side of gametophyte, $\times 6$, showing rhizoids. Archegonia are indicated on the cushion just below the growing point, and antheridia are seen scattered among the rhizoids. spore and the original filament, the growing cells are located in an indentation in its base. From the under surface of this triangular plate rhizoids are formed. The plate, often called the prothallus, is a very delicate structure not often exceeding a square centimetre in area. It has a translucent green colour. It remains a single layer of cells thick except immediately behind the growing region where it becomes several layers thick, and a cushion-like structure is formed.

About the same time as the establishment of this cushion sexual organs begin to be developed. The antheridia are formed first and arise principally on the older narrow parts of the prothallus and on the under side of the cushion. The formation of sexual organs on the prothallus, which has itself developed from a spore, shows clearly that we must regard the prothallus as a gametophyte. The antheridia are almost globular in form and are attached without a stalk directly to the gametophyte. They are composed of a central mass of about forty-eight minute cells enclosed in a rounded cavity formed by two ring-shaped cells lying one on the other, and one plano-convex cell acting as a lid. The nucleus of each of the small inner cells elongates and assumes a spiral form and the cytoplasm clothes it with an exceedingly fine covering of protoplasm which at one end is produced into a tuft of cilia. The absorption of water causes the two ring-shaped cells of the antheridium to swell up unevenly and to distort the en- 
closed cavity to a stellate form. The reduction of the volume of the cavity so produced causes the inner mass of cells to burst through the lid and ejects them from the antheridium. The delicate walls of these latter quickly dissolve, and their contents escape as spiral, ciliated, free-swimming masses of protoplasm. They are the sperm-cells.

The archegonia are formed exclusively on the cushion, close behind the growing region of the gametophyte. They are flaskshaped but much smaller and shorter than those of Marchantia and of Funaria. Their body, or venter, is wholly embedded in the cushion while the short curved neck projects from its under surface and is directed obliquely backwards away from the growing region of the gametophyte. When the archegonium is formed the cells composing the wall of its basal part enclosing the ovum
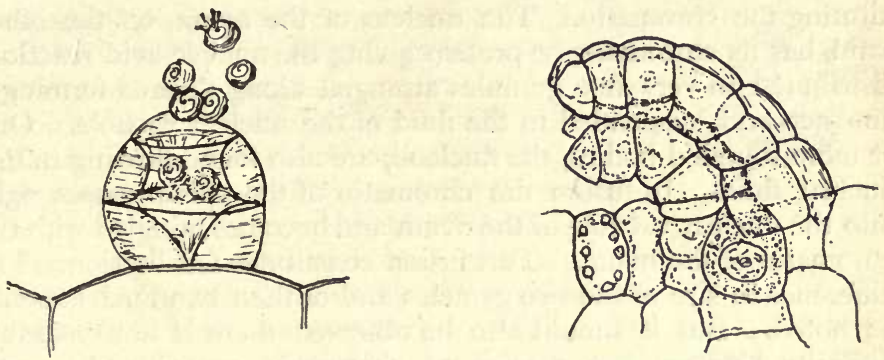

FIG. 42.-Aspidium filix-mas; on left, antheridium discharging sperm-cells ; on right, an archegonium ready for fertilisation, $\times 250$.

do not differ from the surrounding cells of the cushion, and the ovum appears as a spherical cell embedded among the superficial thin-walled parenchymatous cells of the cushion. It is distinguished from its neighbours by its distinct nucleus, by its opaque and finely granular protoplasm and by its poverty in chloroplasts. The neck, which is composed of five rows of cells, projects from the surface immediately over the ovum. The axial core vertically over the ovum is composed of a single prismatic cell, the neckcanal-cell. The four rows surrounding the neck-canal-cell each consists of about five cells. The preparation of the ovum is completed by the formation at its distal pole of a small lenticular cell, the ventral canal-cell. Contact with water will now cause the neck-canal-cell to swell up and burst open the distal end of the neck, and the disintegration and solution of the neck-canal-cell 
and of the ventral canal-cell open a way down to the ovum which lies in the body or venter of the archegonium.

The same supply of water which brings about the ejection and maturation of the sperms will probably open the necks of several archegonia. As in the case of Marchantia and Funaria the sperms are guided chemotactically to the ova. In this case it has been found that malic or maleic acid acts as an attractive substance. Hence it is probable that either of these organic acids is developed in the disintegration of the neck and ventral canal-cells.

The sperms swarm round the lately opened necks and crowd down into their canals. One only-as far as has been observedenters into the ovum and its nucleus fuses with the nucleus of the ovum. Before fusion the two nuclei are very different in appearance. The sperm-nucleus is a spiral band or ribbon of apparently homogeneous protein giving the reaction of nucleic acid and constituting the chromatin. The nucleus of the ovum, on the other hand, has its chromatin, or protein giving the nucleic acid reaction, distributed in very fine granules arranged along threads forming a fine net-work suspended in the fluid of the nuclear vacuole. One or more rounded bodies, the nucleoli, are also found floating in this nuclear fluid. In fusion the chromatin of the sperm passes right into the nuclear vacuole of the ovum and becomes mingled with the chromatin of the ovum. This fusion constitutes fertilisation. The difference in size of the two gametes and of their two nuclei should be noted. But it should also be observed there is not the same disparity between the masses of chromatin contributed to the oosperm by the two gametes.

Immediately after fertilisation the oosperm becomes coated over with a cell-wall. It then divides and becomes a spherical mass of cells. This mass grows rapidly in size and soon bursts the archegonium. Part of it pushes out as a root and grows downwards into the soil, while from the opposite hemisphere, which is turned towards the growing region in the indentation of the parent gametophyte, a first leaf and stem are developed. During the early stages of the growth of these organs, the new plant retains connection with the gametophyte by means of a short, suctorial organ and derives food from it. This organ is called the foot, and is developed from the same hemisphere of the oosperm as the first root. The first leaf soon turns upward, usually through the indentation in the gametophyte, and expands a single leaflet resembling those of the fern-plant in appearance, and having also their opaque green colour unlike the transparent green of the gametophyte. During the development of these organs the nutrition of the young plant is supplied 
through the foot from the parent gametophyte; but when connection has been established between the new plant and the soil by means of the root, and when its carbon supply is secured by its first leaf, food from the gametophyte becomes superfluous and the gametophyte gradually shrivels away. Meanwhile the new plant develops new and larger leaves, its stem waxes stronger, additional roots from its base are produced, and it is soon recognisable as a young fern-plant.

The structure of these organs resembles that of the organs of the mature plant ; the conducting system, however, is less complicated in its formation, and in the young stem appears as a single core of wood surrounded with bast. As the stem strengthens, this core branches and forms the conducting net-work which is characteristic of the mature parts of the stem.

All the leaves of the young plant are at first simply nutritive in their function, supplying carbohydrates by photosynthesis and raising the transpiration-current. Spore-producing leaves-sporophylls-are only produced later.

From the life-history just described, it is evident that the fern-plant is a sporophyte comparable to the sporophyte of Marchantia or of Funaria, but so constructed that it is able to carry on an independent life in all but its initial stages. It is fitted for a wholly terrestrial existence, and we have seen how the complicated structure of its leaves, stems and roots enable it, though living in sub-aerial conditions, to carry

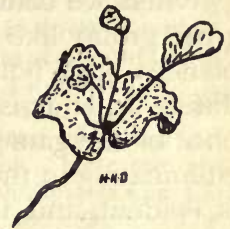

FIG. 43.-Aspidium flix-mas, gametophyte with young sporophyte attached, $\times 2$. on its life-processes, which are similar to those discharged by the submerged organs of aquatic plants.

For the ripening and dissemination of the spores dry conditions are essential. But the germination of the spores and the growth of the gametophyte demand conditions of moisture. Furthermore, the gametophyte resembles the submerged thallophytes in its water requirements more than it does the land-plants. For the successful formation of its gametes and for the process of fertilisation aquatic conditions are imperative. Looked at from this point of view the fern is properly described as amphibious. Its sporophyte generation is distinctly terrestrial while its gametophyte generation is almost entirely aquatic. Comparing its life-history with those of the forms which we have already studied we see that the importance of the sporophyte in the life-cycle is in proportion to the extent to which the plant has withdrawn from aquatic and taken to terrestrial conditions. 
Notwithstanding the much more complex structure of the sporophyte of Aspidium and the great differences between the forms of the gametophytes of the ferns, the mosses and the liverworts, the life-histories of these are evidently similar and the structure of their sexual organs resemble each other in many respects. These broad resemblances have led botanists, in the first instance merely for the sake of convenience, to classify these three sorts of plants together under the name of Archegoniates, all being characterised by the possession of a multicellular archegonium which contains the ovum. These same characteristics distinguish them from the more simplyconstructed Algæ and Fungi which, almost without exception, have their ovum retained in an oogonium of unicellular construction. In contradistinction to the Archegoniates the Algæ and Fungi are described as Thallophytes, a name suggested by the fact that their plant body is never differentiated into stem, root and leaves.

When we compare the different kinds of plants included in a great group like Archegoniates, in addition to the broad resemblances just noticed, a correspondence of the parts in these different plants is forced upon us. For example, the correspondence of the gametophyte of Marchantia, arising from a spore and culminating in the production of sexual cells, with that of Aspidium is evident, and the correspondence of the sporophyte of Funaria with that of Marchantia is equally clear. Again, the theca of Funaria corresponds with the capsule of Marchantia and the archegonium of Aspidium corresponds with the archegonium of Funaria. Such parts of different plants which correspond to one another in their origin, in their relation to the other parts of the plants considered and in their position in the lifehistory, are termed homologues, and such parts are said to be homologous to one another. Homologous parts may, and often do, discharge the same function, but this is by no means always the case; thus the sporophyte of Marchantia does not carry on photosynthesis, while that of Funaria does to a slight extent, and the sporophyte of Aspidium is very efficient in this respect. This difference in function does not, however, prevent these three parts from being homologous. Again, similarity of function does not necessarily indicate homology. This will be readily understood from the comparison of the leaves of the moss, Funaria, with those of Aspidium. They both exercise the function of photosynthesis; but their positions in the life-history are wholly different. The leaves of Funaria are organs of the gametophyte while those of Aspidium belong to the sporophyte. They are therefore not homologous. Such parts performing the same function are said to 
be analogous. Thus homology is a relational similarity while analogy is a similarity of function of parts.

\section{PRACTICAL WORK.}

Sketch the under surface of a fertile, or sporangium-bearing, pinnule of a sporophyll. Observe the indusium and sporangia.

Make a transverse section passing through the sorus. Sketch, showing the placenta, indusium and sporangia.

Scrape off some sporangia approaching maturity into boiled water (to remove the adhering air bubbles) and mount in water. Sketch showing the stalk, annulus, stomium and spores of the sporangia.

Scrape off some sporangia from a ripening sorus on to a dry glass slide. Observe with the low power. Notice the bending backwards of the annulus and the consequent tearing open of the sporangium. Observe the sharp jerks of the recurved annulus.

Gametophytes for examination may be obtained by sowing the spores shed from the leaves on sterilised peat mould. The peat mould is placed in a flower saucer, and moss spores, etc., which it might contain are killed by pouring boiling water over it. The earthenware saucer is covered with a glass plate and is placed standing in a shallow glazed saucer containing water. A good crop of gametophytes may be expected in a month or so.

Take a gametophyte and place it upside down upon a glass slide. Gently remove the adherent particles of peat. Add a drop of water and cover. Observe the colourless rhizoids and the green cells. Examine one of the latter with the high power. Note the numerous chloroplasts and the various stages in their division. Look for antheridıa and archegonia. The former may be recognised by their circular outline and stellate cavity. Careful focussing will reveal the two ring-shaped cells. Often a mass of spermmother-cells may be seen in the antheridium and the beginning of the motion of the sperms may be detected within them.

The archegonia are less easily recognised, chiefly because they are sunk in the thicker part of the gametophyte. From the surface aspect of the preparation only two of the four parallel rows of cells forming the neck are at once visible. A dark brown streak between them indicates the neck-canal leading down to the ovum. Focussing down along this brings the cavity of the venter and the ovum into view.

Reconstruct the shape of an archegonium from a series of optical sections taken in the neck and the venter.

Sketch an older gametophyte with young sporophyte attached. 


\section{LECTURE XXI.}

THE homology of parts and the correspondence of stages in the life-histories of two divergent organisms are often brought to light or made more apparent by comparison with a third organism which is intermediate between the two. These intermediate organisms are often spoken of as links or synthetic forms.

It is owing to its character as such a connecting-link between the Ferns on the one hand and the Seed Plants on the other that the study of the structure and life-history of Selaginella is 'of particular interest.

There are large numbers of different kinds of Selaginella. Only

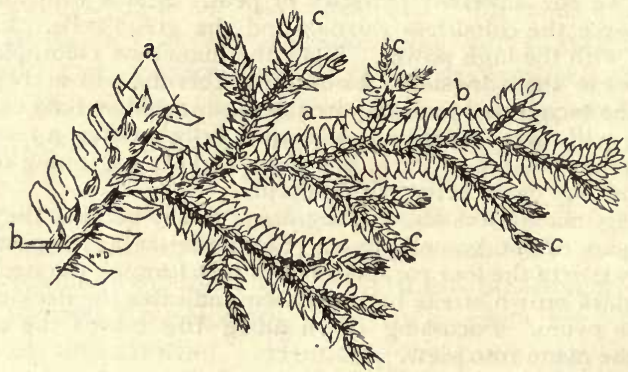

FIG. 44.-Selaginella martensii. $a$, lateral leaves; $b$, dorsal leaves; $c$, cones.

one of them is native to the British Isles. As it is rare and diffcult to obtain, our study will be carried out on a tropical species, Selaginella martensii, which is usually grown in green-houses in this country.

This Selaginella is a low-growing, delicate and moss-like plant. Its white translucent stems lie prostrate on the soil, branching only in one plane-that parallel to the surface of the soil. The ends of the branches are closely covered with green leaves. The leaves are oval in outline, but pointed. Their edges are serrated. A midrib runs from the tip to the base of each. They are attached directly to the stem, having no stalk. They are distinctly of two sizes and 
are arranged in four rows down the stem. Two rows of small leaves are along the upper or dorsal aspect of the stem and there are two rows of larger leaves, one along each side. It may also be seen, especially in the older parts of the stem where the leaves are more widely spaced, that the leaves occur in pairs on the stem-a large lateral leaf and a small dorsal leaf on the other side being exactly opposite and forming a pair. 'The leaves of adjacent pairs are on alternate rows. Closer examination with a lens shows a minute shell-like scale on their upper surfaces very near the base.

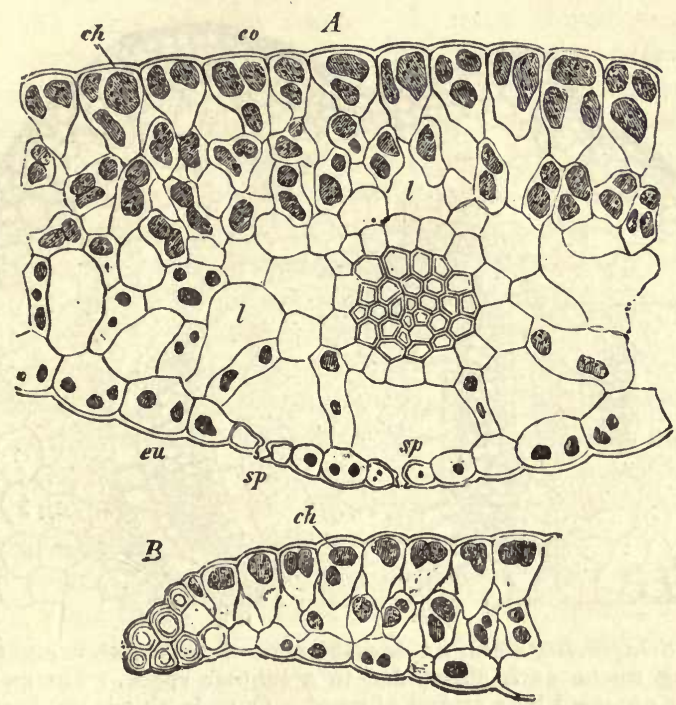

F IG. 45.-Selaginella, leaf, transverse sections, $\times 300$. eo, upper, $e u$, under epidermis; $l$, air-spaces; $s p$, stomata. (From Evans' An Intermediate Textbook of Botany. After Goebel.)

This inconspicuous organ is called a ligule. It is to all appearance functionless. It is rarely found on the leaves of living plants, but is of interest inasmuch as it characterises the leaves of important groups of fossil plants. In Selaginella it may be regarded as an heirloom, proving the antiquity of its descent. At the bifurcations of the stem sometimes a stiff wiry organ is found directed from the under surface of the stem towards the soil. It is a rhizophore. From its slightly bulbous end thread-like roots grow and penetrate into the soil. In some specimens the ends of some of the branches tend to turn upwards and assume a vertical position. The leaves on these 
vertical branches, or cones, are all similar in size and are distributed uniformly round the stem. In the angles between the leaves and the stem are seen minute whitish globular bodies which are sporangia.

In structure the small leaves resemble those of a fern rather than those of a moss, with which we might at first be inclined to compare them. They have a thick mid-rib, and on either side of it the green tissue is several layers of cells thick and, as in the fern, the mesophyll towards the under surface is more loose and

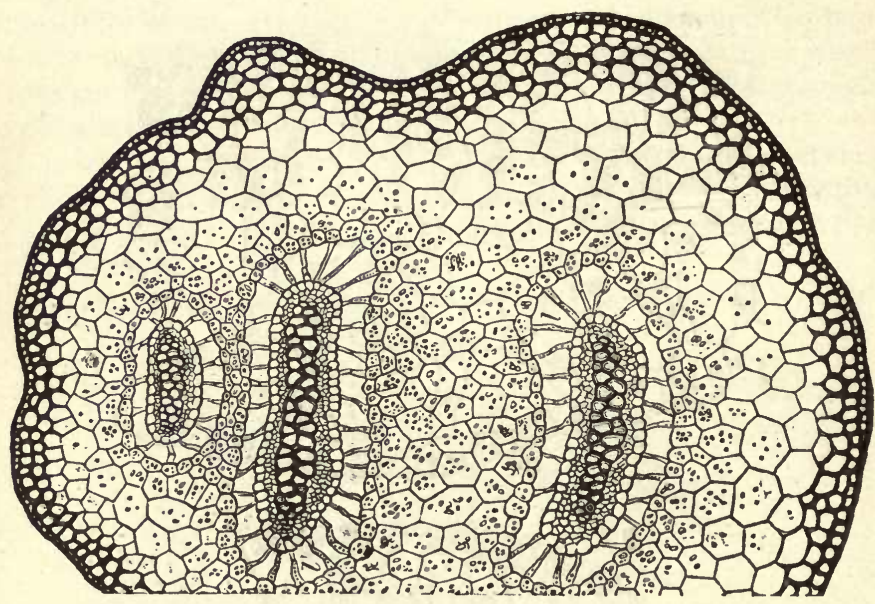

Fig. 46.-Selaginella, stem, transverse section, $\times$ I50, showing three conducting tracts, each suspended in a tubular space. The axis of each tract is occupied by a strand of wood. Outside this is the bast. (From Evans' An Intermediate Textbook of Botany. After Sachs.)

spongy than that above it. It is covered over on both sides by an epidermis and this sheath of cells is perforated on its under side by stomata. The outer walls of the epidermis are covered with cuticle; the remaining walls of the epidermis and of the mesophyll are thin. In each cell of these tissues there are one or two large chloroplasts, a state of things very unlike the other ferns and strangely reminiscent of the Algæ.

The structure of the stem is peculiar. It is a shaft of fundamental tissue traversed lengthwise by a tubular passage and covered over by the epidermis. Running down the tube is a vascular tract slung in its axis by elongated cells which are con- 
nected with the inner surface of the tube and take their origin from the outside of the conducting tract. The conducting tract resembles one taken from Aspidium. It consists of a strap of wood oval in cross section encased in a sleeve of bast. The greater part of the strap of wood is made up of scalariform tracheids, one or both of its edges are occupied by a thread of spiral and annular tracheids. These form the protoxylem, and if we examine a conducting tract near to the growing end of a branch we will find that the protoxylem threads are differentiated before the rest of the wood which develops subsequently towards the axis of the tract. A tract developing in this manner, the protoxylem being at the outside, is called exarch. These exarch conducting tracts are not uncommon among the Ferns but are seldom found in the stem among the Seed Plants. The bast contains sieve-tubes like those of Aspidium. The elongated cells suspending the conducting tract in the tube formed by the cortex correspond to the endodermis of Aspidium.

The structure of the rhizophores and roots resemble in a general way that of the stem, except that the cortex fits closely on the conducting tract, and there is little or no trace of the cavity surrounding the tract. Further, there is but one thread of protoxylem in the wood and it is laterally placed. In addition to these differences the root carries a root-cap of loose cells covering its growing apex. The growing region of the rhizophore is naked while that of the stem is covered over by the young leaves.

The leaves of the cones have a structure similar to those on the prostrate stems, but in the angle formed between them and the axis of the cone there is a single globular sporangium, attached to the angle by a short stalk. When the sporangia are mature it is seen that they are of two kinds: the smaller are smooth and eggshaped; they are called microsporangia, while the larger, which are trilobed, are called megasporangia. Each kind breaks open along a line of weakness running on the sporangium at right angles to the direction of the mid-rib of the subtending leaf, or sporophyll, splitting the sporangium in half. From the microsporangia large numbers of microspores are shed, while from the megasporangia four megaspores come. Both the microspores and megaspores show from their shape that they have been formed in tetrads in a common mother-cell, being tetrahedral in form and having three flat converging sides and one rounded or spherical side. As a matter of fact the early stages in the development of the two kinds of sporangia are identical. Each originates as a stalked globular mass of sporogenous cells covered over with two layers of cells 
which become the wall of the capsule. The central globular mass now forms a layer of cells lying against the inside of the wall. This layer is called the tapetum inasmuch as by the products of its deliquescence the spores are nourished. In the microsporangium each of the sporogenous cells becomes rounded and by two successive divisions gives Irise to four, spores. The remains of the mother-cells and of the tapetum are; used up in the development

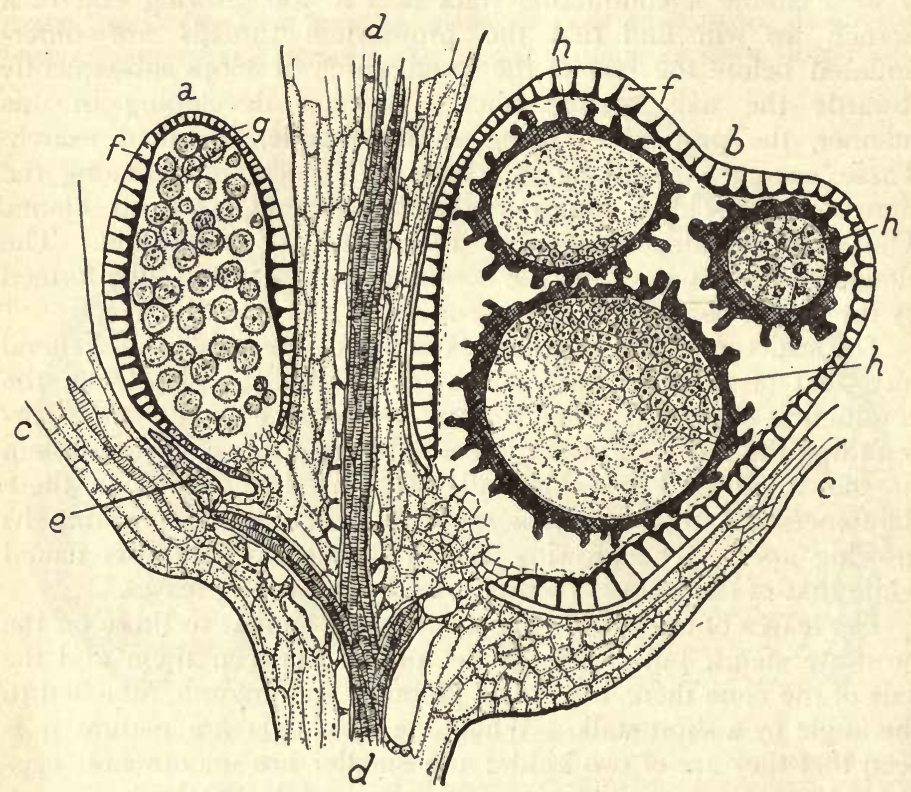

Fig. 47.-Selaginella, base of cone, longitudinal section, $\times 80$. a, microsporangium; $b$, megasporangium ; $c$, sporophyll ; $d$, axis of cone; $e$, ligule; $f$, wall of sporangium; $g$, microspores; $h$, megaspores. (After Chamberlain.)

of these spores and in the formation of their thick and warty coat. The development of the megasporangia is quite similar to that of the microsporangia except that only one mother-cell divides and gives rise to spores, while the others and the tapetum are absorbed by the growth of these four which attain a much greater size than the microspores; the microspores being about $0.025 \mathrm{~mm}$. and the megaspores about $0.55 \mathrm{~mm}$. in diameter.

The germination of the spores may begin before they are shed 
from the sporangium, but it is usually continued on the moist soil. The microspore begins by its division into two very unequally sized cells, the larger one practically occupying the whole spore while the smaller one is very minute. This latter one undergoes no further development and is the only somatic or vegetative cell of this reduced gametophyte. The large cell segments and produces a layer composed of eight cells lying against the inside of the spore coat and a mass of rounded cells occupying the space surrounded by the eight. Within each of the cells forming the central mass a sperm develops in a manner resembling the de-
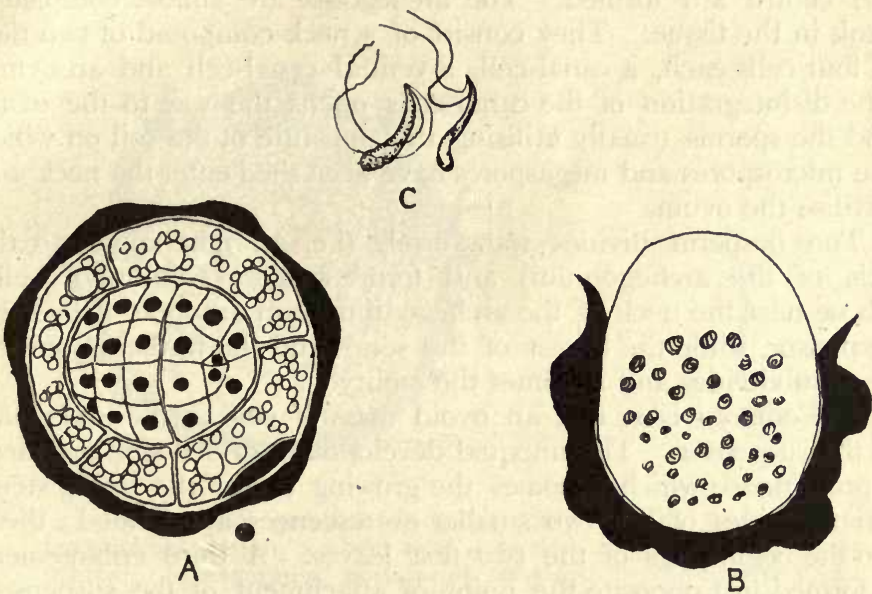

Fig. 48.-Selaginella, germination of microspores. A, section of microspore showing sperm-mother-cells surrounded by the cells of the antheridium. The blackened cell below on the right is the somatic cell of the gametophyte. B, bursting of the spore-coat and liberation of the sperms. A and $\mathrm{B}, \times$ about I250; C, more highly magnified. (After Lyon.)

velopment of the sperms of the Ferns and Mosses, but the sperm of Selaginella is club-shaped with a corkscrew bend in it, and it possesses two cilia only, resembling in this respect the sperms of the Mosses and not those of the Ferns. When the sperms are approaching maturity the cells forming the enclosing capsule and the remnants of the sperm-mother-cells disintegrate and become mucilaginous. By large absorption of water they swell up, burst the spore-coat, and set free the sperms.

Meantime the germination of the megaspores is taking place. The original single nucleus of the spore divides repeatedly and its 
segments aggregate in the protoplasm immediately within the three converging flattened sides of the spore. Cell-walls are formed dividing this protoplasmic mass into cells each containing a nucleus, and thus a tissue is formed at the apex of the spore. Later on, by further nuclear divisions followed by the formation of cellwalls, the whole body of the spore is filled with tissue. The growth of this gametophyte bursts open the apex of the spore and exposes the first formed tissue within the spore. In this tissue the archegonia are formed. The first to arise are centrally placed on this exposed tissue. If they fail to be fertilised others further from the centre are formed. The archegonia are almost completely sunk in the tissue. They consist of a neck composed of two tiers of four cells each, a canal-cell, a ventral canal-cell and an ovum. The disintegration of the canal-cells opens the way to the ovum and the sperms usually utilising the moisture of the soil on which the microspores and megaspores have been shed enter the neck and fertilise the ovum.

The oosperm divides transversely (i.e. at right angles to the axis of the archegonium) and forms a short column of cells. Those next the neck of the archegonium form an organ called the suspensor, while the lowest of the series ard the furthest from the neck subdivides and becomes the embryo.

The embryo is at first an ovoid mass of cells at the lower end of the suspensor. The unequal development of one side produces a prominence which becomes the growing end of the young stem. On the sides of this two smaller excrescences are formed; these are the beginnings of the two first leaves. A third enlargement is formed just opposite the point of attachment of the suspensor. This becomes the foot which acts as an haustorial organ and absorbs the food material stored in the gametophyte. Later on the first root arises in the tissue between the foot and the suspensor. Very soon the growth of the embryo causes it to burst out from the gametophyte and the containing spore coat. Its root grows down into the ground to absorb water and the necessary mineral supplies, while the stem pushes forth producing leaves to carry on photosynthesis and transpiration, and thus the young sporophyte is launched.

Comparison of Selaginella with Aspidium shows that the gametophyte of the former is much simpler in its construction than that of Aspidium. The vegetative apparatus of the male gametophyte is limited to the single small cell formed by the first division of the microspore, and this corresponds to the whole prothallus of Aspidium. The small capsule of cells surrounding the sperm- 
mother-cells is a single antheridium formed on this diminutive prothallus. The whole gametophyte is thus practically reduced to a single antheridium. Having no vegetative organs it is entirely dependent on store materials laid up in the spore for its support.

Although not quite so diminutive as the male, the female gametophyte of Selaginella is very inconspicuous compared to that of Aspidium. It often forms a few rhizoids from the exposed part of the prothallus but neither these, nor the small amount of green cells also formed in this region of the prothallus, are very effective nutritive organs, and consequently the female gametophyte is

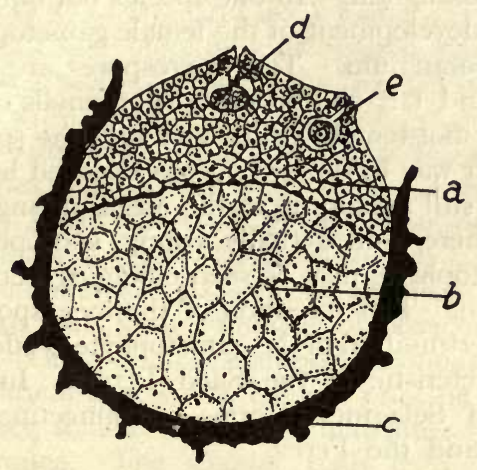

FIG. 49.-Selaginella, germinating megaspore, longitudinal section, $\times$ about roo. $a$, reproductive; $b$, vegetative portion of female gametophyte ; $c$, spore-coat; $d$, embryo; $e$, ovum in archegonium. (After Lyon.)

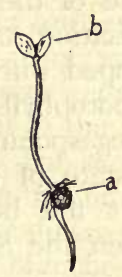

FIG. 50.- Selaginella martensii, young s porophy te. $a$, megaspore; $b$, first leaves (cotyledons).

almost as completely dependent on the materials stored in the spore by its parent sporophyte as is the male gametophyte.

The differentiation of the gametophyte generation into male and female individuals is a complication not found in Aspidium. However, it should be mentioned that often small prothalli of Aspidium are found which develop antheridia only. Probably insufficient nutrition in these cases has precluded the formation of the cushion on the prothallus and consequently there has not been sufficient thickness to allow of the development of the archegonia. Thus the sexual differentiation of Aspidium is probably due to external conditions. But we have in Marchantia an example of sexual differentiation which is as complete as that of Selaginella. In Marchantia, however, the spores which produce the male and 
female gametophytes are indistinguishable, and it is possible that external conditions decide the sex of the plant. In Selaginella the external difference of the spores at the time of shedding shows that the sex of the gametophyte is already decided in the spore, and external conditions are powerless to alter it. Plants which are thus characterised by two kinds of spores, each giving rise to sexually distinct gametophytes are called heterosporous. They are found among the higher fern-like plants; and all the Seed Plants are heterosporous.

It has already been noticed that the spores of Selaginella begin their germination in the sporangium. In one species not only the germination but the whole development of the female gametophyte takes place in the megasporangium. The microspores are shed from the microsporangia and fall on the lower sporophylls of the cone. When the cone is moistened with dew or rain the sperms are liberated and make their way to the archegonia exposed by the rupture of the megaspores still remaining in the megasporangium. Fertilisation takes place there, and actually young sporophytes, developed within the gametophyte, may be seen emerging between the sporophylls of the cone. The retention of the megaspore in the megasporangium until fertilisation of the gametophyte is effected is a state of things characteristic of the Seed Plants. In this respect the heterospory of Selaginella forms a connecting-link between the Seed Plants and the Ferns.

\section{PRACTICAL WORK.}

Sketch a piece of Selaginella martensii showing the prostrate branching system, the arrangement of the leaves, the rhizophores and roots.

Make transverse sections of the stem, and record your observations on them in sketches with the low and high power.

Mount several leaves in water. Look for the ligule. Examine the under surface of the leaf and note the stomata, and the presence of single chloroplasts in some of the cells.

Pick off some of the cones and boil them in dilute potassium hydrate to clear them. Mount them in water and examine. The microsporangia and megasporangia will be seen, and the ligule on some of the sporophylls.

Cut longitudinal sections of the cones and supplement with these your observations with the cleared specimens.

Shake out some of the spores on to a piece of filter paper in a Petri dish. Moisten with rain-water and keep in a warm room for a few weeks. Examine at intervals. Notice the bursting of the spores and the female gametophyte protruding from the megaspore and later the stem and first two leaves and the root of the embryo sporophyte. 


\section{LECTURE XXII.}

WE have seen that Selaginella is in many ways a connecting-link between the Ferns and Seed Plants. The truth of this statement will be much more fully recognised when a study of the structure and life-history of some of the Seed Plants has been made.

We will begin our study with the examination of the Scots Pine, Pinus silvestris. This tree forms forests in northern European countries, but is found scattered in mountainous parts of the British Isles. Its stout woody stem, heavy branches and strong roots form a great contrast to the corresponding parts in the common Ferns. Its stem tapers from base to summit and at intervals puts forth a number of branches all round it at the same level. These branches support secondary branches. The secondary branches, however, emerge only on the sides of the primary branches, so that the lateral branching systems have a dorsiventral appearance. The slender ends of the stem and branches are clothed with green leaves. Examination of these ends show that they terminate with a bud and that they are covered over with large numbers of small scale-like leaves arranged in spirals upon their surfaces. In the axils of each of these scales is a tuft of two green needle-shaped leaves. The bases of the two green leaves are continuous with, and attached to, a very short shoot or branch inserted in the angle, or axil, formed by the scale with the supporting stem. The attachment of the green leaves to the top of the dwarf shoot is concealed by a number of diminutive scales which are fixed round the base of the leaves close to the top of the dwarf shoot. The dwarf shoot has a limited life only, and after producing its two green leaves it ceases to grow, and it is detached from the stem and falls off after three or four years. While the greater part of the slender terminations of the stem and its branches are covered over with scales including dwarf shoots, larger buds like that at the apex are formed in the axils of the scales at the base of each season's growth. These grow out into long shoots and are the origins of the groups of branches on the main stem. Similarly a pair of large buds which produce branches are formed 


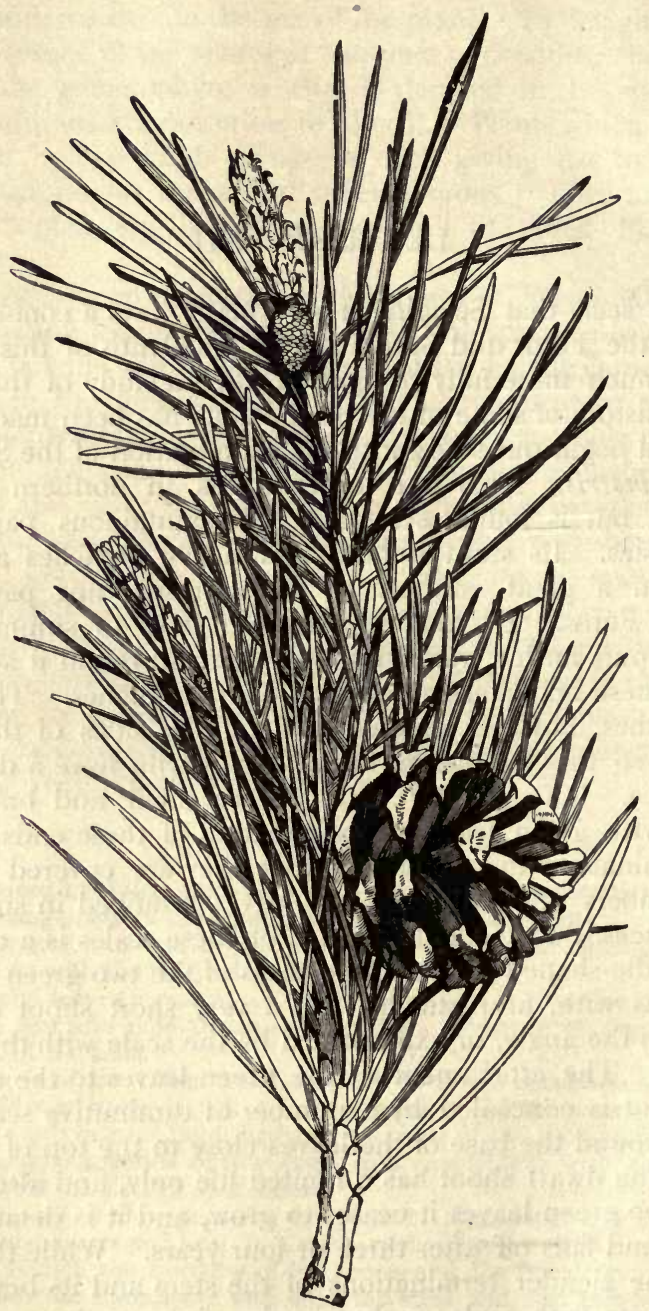

FIG. 51.-Pinus silvestris, end of branch with seed-cone of this year and one of the year before last. (From Evans' An Intermediate Textbook of Botany.) 
at the base of each season's growth at the end of the lateral branches and thus provide for the dorsiventral branching of the lateral branches.

The stem is prolonged downwards into the soil by a large root. The downward extension of this tap-root depends on the depth of the soil. It sends off side roots and these branch repeatedly. The finest branches of the roots are delicate and thread-like.

The green, or foliage, leaves are long and narrow. Each is a half cylinder in form and tapers towards both ends. Its flat surface at the base is turned towards its neighbour in the tuft, its upper part is loosely twisted. A single vein runs down the middle of each leaf containing two small vascular bundles, or

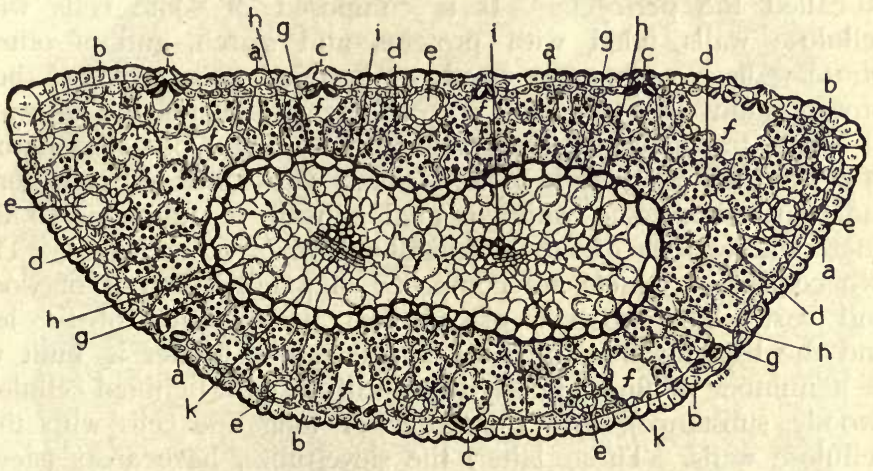

Fig. 52.-Pinus silvestris, leaf transverse section, $\times 58$. $a$, cuticle; $b$, epidermis; $c$, stomata; $d$, mesophyll-cells with infolded walls; $e$, resinducts; $f$, air-spaces; $g$, endodermis ; $h$, transfusion tissue; $i$, wood or xylem; $k$, bast or phloem.

conducting tracts. Outside the vein is the mesophyll of the leaf, and it is protected by a fibrous layer, which is in turn coated by the epidermis. The epidermis is composed of thick-walled cells forming strong fibres elongated in the direction of the length of the leaf. The outer walls of its cells are covered with a firm cuticle. The epidermis is perforated by longitudinal rows of stomata, which lead into air-cavities in the mesophyll. The stomata are bordered, as in the case of the Archegoniates, by two guard-cells. These are sunk below the general level of the epidermis, their walls are much thinner than those of the other epidermal cells and they contain numerous chloroplasts, while the other cells have none. The mesophyll-cells are peculiar, they are isodiametrical and fit fairly closely together. From their thin walls 
incomplete partitions extend into the cavity of the cell, dividing the cell up into a number of incomplete compartments. In these cells are found large numbers of chloroplasts embedded in the film of protoplasm lying against the wall. Between the isodiametrical mesophyll cells and the epidermis there is a sheet of elongated fibres which is continuous under the epidermis, except below the stomata, where it is replaced by V-shaped mesophyll cells enclosing between their two branches the cavity which leads through the stoma to the outside. The mesophyll is bounded on its inside by a uniform layer, one cell thick, the endodermis. Its cells are oblong and fit closely together. The tissue between the conducting tracts and the endodermis is called the pericycle. It is composed of some cells with cellulose walls filled with proteins and starch, and of others whose walls have become woody or lignified and have lost their protoplasmic contents and are, in fact, tracheids. The walls of these tracheids are furnished with bordered pits, the nature of which we will study later. These two kinds of cells form the transfusion-tissue, which is analogous in its functions to the finer ramifications of the vascular bundles of other leaves. The two conducting tracts are composed of parallel strands of wood and bast. The wood is placed next the flat side of the leaf and the bast towards the convex side. The former is built up of a number of fine capillary-tubes with walls of lignified cellulose (woody substance), and the latter of long fine cells with thin cellulose walls. These latter, the sieve-tubes, have areas (sieveplates) on their walls perforated with very minute pores, and through these the mucilaginous contents of the sieve-tubes are continuous. The two conducting tracts run down the leaf enclosed in the transfusion-tissue and pass without any marked change in their direction into the dwarf shoot. Here the four strands contributed by the two leaves remain separate for a short distance, but gradually converging, they soon fuse together and form a tube surrounding the narrow pith. This tube formed of the downward prolongation of the conducting tracts of the leaves enters the cortex of the stem which supports the dwarf shoot obliquely. As it approaches the pith it divides into two strap-like portions which bend downwards and run in a steep spiral on the outside of the pith towards the base of the stem. In their course downwards the two straps fuse with the bundles coming from other dwarf shoots. Owing to these fusions, the number of bundles in the stem at any level, except at the very top, is less than the sum of the bundles contributed by the dwarf 
shoots above. In fact the number seen in any cross section varies from six to fifteen. The wood of the conducting tract, which is towards the flat surface of the leaf, is placed on the inside of the tract when the latter turns downward in the stem and the bast lies on its outer side. In the leaf the bast lies directly against the wood, but in the stem a thin layer of growing cells - the cambium - separates the two.

The tissue of-the stem in which the conducting tracts are

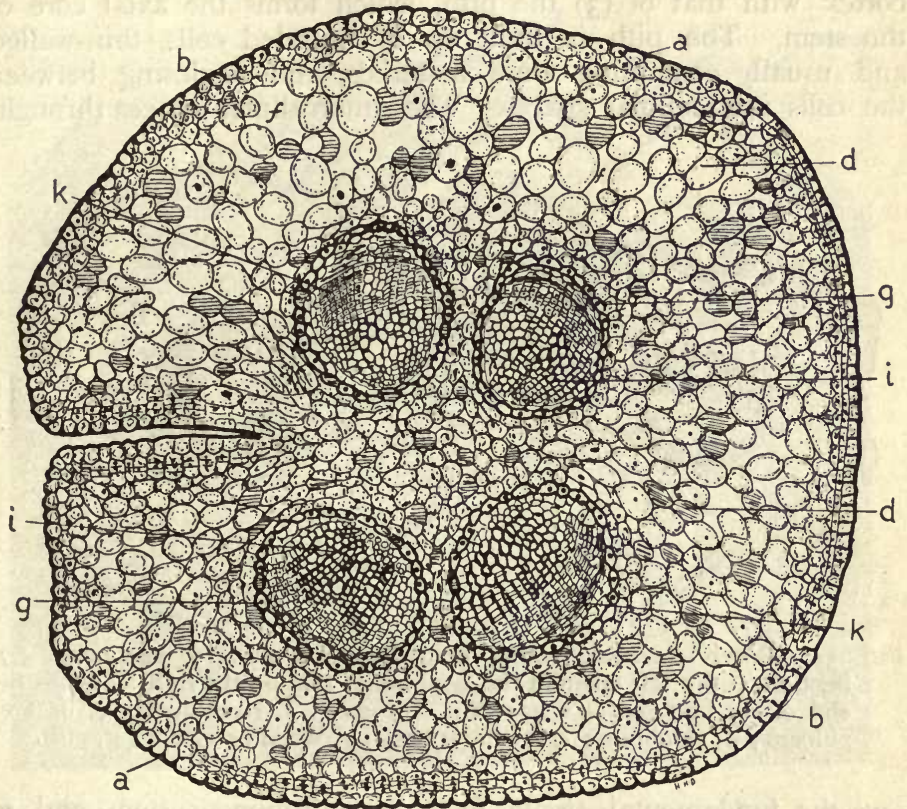

FIG. 53.-Pinus silvestris, apex of short shoot, transverse section at the point of attachment of the two leaves, $\times 5^{8}$. $a$, cuticle; $b$, epidermis ; $d$, cortex; $g$, endodermis ; $i$, wood or xylem.

embedded is called the fundamental tissue. It is limited on the outside by the single layer of cells forming the epidermis, which is continuous over the whole surface of the end parts of the branches, the dwarf shoots and their leaves. The tough outer layers of its outer cell-walls form a nearly impermeable sheet-the cuticle-coating the whole; this is only perforated by the stomata. Within the epidermis the fundamental tissue is divisible into three regions: (I) Outside the conducting tracts 
is the cortex which is composed of cylindrical cells with intercellular spaces between them, their axes being parallel to the axis of the stem. They are thin-walled and the outer cells contain chlorophyll. Starch grains are usually found in all. Between the conducting tracts and separating them from one another are (2) the medullary rays. They are composed of cubical or oblong cells with thin walls, containing stores of carbohydrates. They connect the fundamental tissue of the cortex with that of (3) the pith, which forms the axial core of the stem. The pith is built up of rounded cells, thin-walled and usually containing store materials, and enclosing between the cells intercellular spaces. The intercellular spaces through-

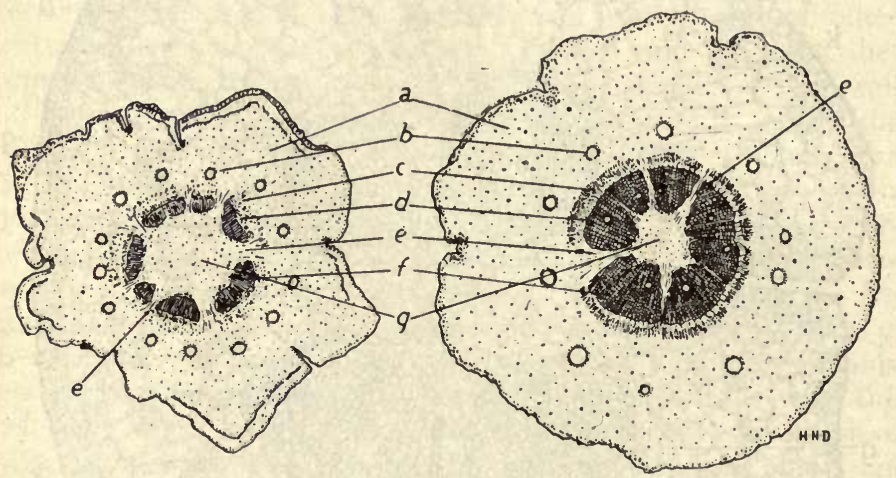

FIG. 54.-Pinus silvestris, young long shoots, transverse sections, $\times 7$. Section on the left taken at the beginning, that on the right towards the end of the first season's growth. $a$, cortex; $b$, resin-ducts ; $c$, bast or phloem ; $d$, cambium; $e$, medullary ray; $f$, wood or xylem; $g$, pith.

out the fundamental tissue form a continuous system and so allow of a slow interchange of gases between the inner tissues and the outside. Throughout the stem and leaves there is another system of passages in the form of branching-tubes lined with cubical cells which exude resin into the tubes. These resin-passages are found in the wood, in the medullary rays and in the cortex of the stem and of the mesophyll of the leaf. Being surrounded by turgid cells the resin in these ducts or passages is under pressure, and when the tree is wounded is extruded through the surface of the wound. In these cases the resin appears to act as an antiseptic and coating the wound prevents excessive loss of water by evaporation, 
The stem grows in length at its tip. This tip is rounded and is formed of small thin-walled cells, which fit closely together. They are isodiametrical and polyhedral in form. Protoplasm fills their cavities completely, and the nuclei of these cells are comparatively large. At the extreme tip the cells divide at short intervals and so their numbers increase rapidly. They all resemble each other and are quite undifferentiated. At a very short distance below the tip small excrescences on its

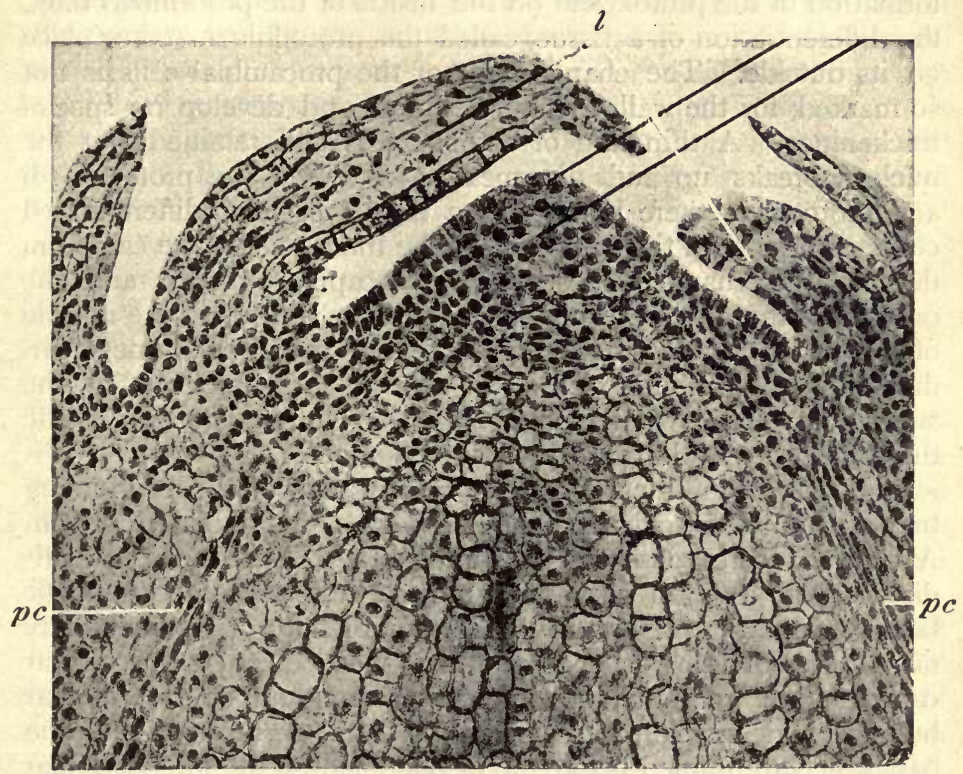

Frg. 55.-Pinus silvestris, growing tip of stem, longitudina section. $l$, young leaves ; $p c$, procambial tracts. (From Evans' An Intermediate Textbook of Botany.)

side are developed. These are the beginnings of the scales and of the dwarf shoots. At first the cells of these resemble those of the extreme tip, being quite undifferentiated from one another. Later on some of the deeper cells of the short shoots and of the tip of the stem, which supports them, elongate and divide longitudinally only, thus forming tracts running from the scales and dwarf shoots into the apex, or tip, of the stem. These are the procambial tracts and are the forerunners of the vascular 
bundles. The first part of the bundle to be differentiated is the innermost part of the wood. To form it the inner cells of the procambial tract elongate and become tubular, spiral and annular bands are laid down inside their walls while the protoplasmic contents disappear and the walls become lignified. The spiral and annular tubes developed first from the procambium form a continuous thread of tissue called the protoxylem, running on the inner side of the wood strand. Nearly as soon as the formation of the protoxylem on the inside of the procambial tract, the differentiation of a tissue called the protophloem takes place on its outside. The change here of the procambial cells is not so marked, for the walls remain cellulose and develop no special thickenings. A thin film of cytoplasm is still retained, but the nucleus breaks up and disappears. Between the protophloem and protoxylem there is left over. a thin layer of undifferentiated cells. These form the cambium. The inner cells of the cambium develop the characteristics of woody capillary tubes, and the outer cells those of sieve-tubes, while the cells forming the middle of the layer divide longitudinally and give rise to new undifferentiated cambium cells. In this way the thickness of the strand of wood is increased by additions on its outside, while the bast receives additions on its inner surface.

The growth in thickness of the two strands of the conducting tract naturally stretches the medullary rays in a radial direction. Apparently in response to this stimulus the cells of the rays, equidistant from the pith with the cambium in the conducting tracts, begin to divide, and to add to the radial length of the medullary rays. This dividing layer in the rays is called the interfascicular cambium, in contradistinction to the cambium of the vascular bundles, which is called fascicular cambium. The wood and the bast which are formed before the development of the interfascicular cambium are called primary wood and primary bast. Wood and bast formed subsequently to that stage are secondary. The fascicular and interfascicular cambium now form a continuous sheet outside the wood intersecting the medullary rays. This sheet lays down on its inner surface wood-tubes and medullary-ray-cells, and on its outer surface sieve-tubes and medullary-ray-cells. These new elements sometimes correspond to those already laid down in the primary arrangement of tissues, but sometimes they do not. In the latter case medullary-ray-cells laid down radially opposite the primary wood strands initiate a ray which does not extend as far as the pith and does not reach the cortex. Such rays are called secondary, in contradistinction to those which extend from the 
pith to the cortex, which are the primary rays, and persist from the primary arrangement of tissues obtaining before the differentiation of interfascicular cambium. The secondary medullary rays of Pinus are usually only a single layer of cells thick and from one to ten, or even fifteen, cells deep. The interfascicular cambium forms woody tubes in the primary medullary rays. The primary rays are thus reduced in thickness, as they extend outwards, keeping pace with the thickening of the wood, and soon become, like the secondary rays, only one cell thick. The reduction in the size of the primary rays by the addition of secondary wood converts the separate woody strands, such as we have seen are found near the apex, into a continuous woody cylinder a little lower down. This change is externally visible in the greater rigidity and hardness of the stem at this level, compared with its succulent and herbaceous consistency nearer to the tip.

A considerable amount of secondary wood may be formed by the close of the first growing season. Thus, if at the end of the summer we cut across a tip developed during the previous spring, we will find the woody cylinder already consolidated into a rigid tube. The primary wood forms ridges running down the inside of this tube and the protoxylem threads occupy the crests of these ridges. The position of the protoxylem on the inner aspect of the woody strands is characteristic of the wood of the stems of Seed Plants. Such wood strands are called endarch and contrast with those of the Ferns in which the protoxylem is often surrounded by, and embedded in, the woody strand (mesarch).

As the season progresses the woody tubes or tracheids formed by the cambium are narrower than those formed earlier in the season. At the same time their walls are thicker. At the end of the summer the activity of the cambium ceases. Cell division is suspended and no differentiation of cambial cells into tracheids and sieve-tubes takes pläce. However, with the return of spring growth recommences. The cambium-cells grow in thickness and they divide longitudinally. At the same time differentiation begins among the cells forming its inner and outer layers. The former become tracheids and the latter sieve-tubes. The tracheids which are formed in the spring are much wider than those formed in the summer and their walls are thinner. The cambium adds tracheid after tracheid to the wood, and as the season advances the radial diameter of the tracheids successively added becomes gradually smaller and their walls thicker. In this way the layer of wood formed during each growing season gradually changes in character; that formed in the spring is built of wide tubes with thin walls, 
and passes gradually into that formed in the late summer which has thick walls and narrow tubes. The latter is denser, there being more wood substance in it. The recommencement of growth in the spring abruptly starts with the large tubes, which form porous wood.

The succession of layers of wood formed in the successive seasons is clearly marked out by this abrupt change from late summer to early spring wood. So when a piece of pine wood is cut across you will see a succession of light and dark concentric zones. The light zones are the spring wood and pass outwards gradually into the dark zones which are the summer wood. Outside each dark zone there is an abrupt change and on its outside comes the spring wood of the next year. Evidently this zonation enables you to tell the age of a branch or stem when cut across,

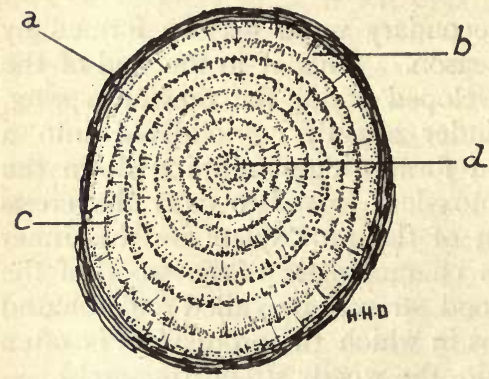

FIG. 56.-Pinus silvestris, cut surface of branch nine years old. $a$, bark; $b$, spring wood; $c$, summer wood; $d$, pith. each year being represented by a ring in the cross section. These year-rings are not of uniform thickness and their comparative sizes constitute a record of the history of the tree, and shows how the succeeding years were favourable to growth or the reverse. In longitudinal sections of the wood these zones appear as parallel bands, each zone representing a hollow tube of wood. If the longitudinal section is radial, i.e. running through the axis of the stem, the spacing of the parallel bands depends directly on the thickness of the year-rings; if, however, the longitudinal section does not pass directly through the axis it is tangential, i.e. tangential to some cylindrical surface concentric with the pith, and the spacing of the parallel bands is normally wider near the middle line of the section. In oblique tangential sections evidently the year-rings will appear as ellipses, and in this way they will produce the beautiful figure seen in panels of wood cut tangentially and slightly obliquely in the stem.

The structure of the roots differs somewhat from that of the stem. In them a single axial procambial core of elongated cells gives rise to the conducting tracts. The protoxylem is differentiated at a few positions on the surface of this cylindrical core, and 
the protophloem is also formed on its surface, at places alternating with the groups of protoxylem. The primary wood differentiates in the central part of the procambium and forms a core of wood connecting the protoxylem threads. The protoxylem groups thus lie on the outside of the primary wood. The woody core of the root is consequently described as exarch. In the pine there are usually three groups of protoxylem, so that the primary-wood-core is a three-sided prism with protoxylem threads upon its angles. On the flat, or slightly grooved, sides of the prism are the threads

\section{$\begin{array}{llllll}P & C x & C & X y & \text { En }\end{array}$}

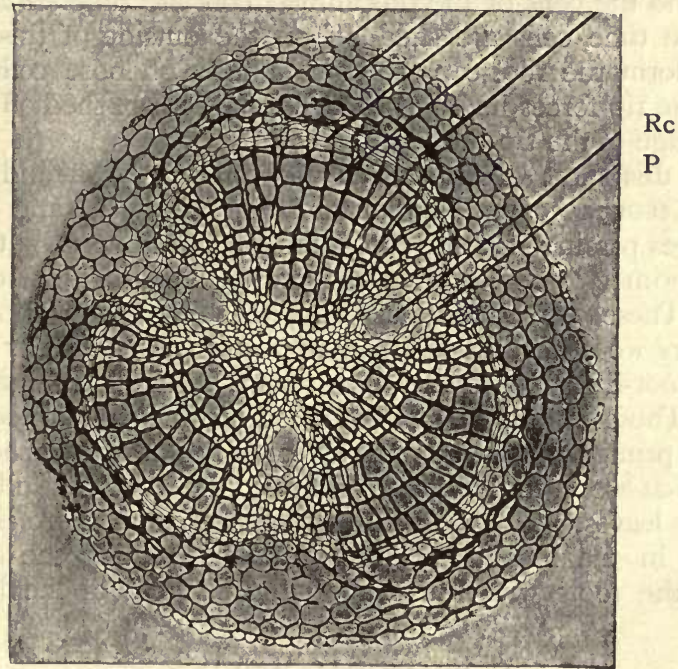

Fig. 57.-Pinus silvestris, transverse section of root, $\times 40 . \quad P$, piliferous - layer; Cx, cortex; En, endodermis; P, phloem; C, cambium; Xy, xylem; Rc, resin-canal. (Photomicrograph by Flatters, Milbourne, \& McKechnie.) (From Evans' An Intermediate Textbook of Botany.)

of protophloem. Between them and the wood is the cambium. While the primary wood is differentiating the cambium forms primary bast on the inside of the protophloem and, by the formation of tracheids on the flat face of the woody prism, converts it into a cylindrical core of wood. Interfascicular cambium now forms outside the protoxylem threads, and unites the cambium into a continuous sleeve ensheathing the core of primary wood and protoxylem. The formation of secondary wood by this continuous cambium increases the thickness of the woody cylinder, just in the same 
manner as the activity of the cambium of the stem adds to the thickness of the wood in that organ.

The arrangement and growth of the tissues outside the conducting tracts is much the same in the root as in the stem, except that near the tips the outermost layer of all contains certain cells which elongate themselves at right angles to the surface and grow out as tubular cells into the surrounding soil. These are the root-hairs.

The tip of the root is composed of growing cells like those of the extreme apex of the stem, and from them the cells of the cortex and the cells of the procambial core are differentiated. At the same time cells are shed off on the outside of this growing tip and form the root-cap, which is a mass of loose cells through which the tip forces its way. The cells which are shed off lubricate the passage of the tip through the soil.

From this short description of the origin, growth and arrangement of tissues you will understand that the water and dissolved substances passing into the root-hairs are transmitted by the cortex to the primary wood and to the protoxylem in the ends of the roots. These are continuous with the outer and more recent secondary wood next to the cambium and pass the water collected by the root-hairs up to the most recent parts of the wood of the stem. These outer layers of the wood of the stem are continuous with the primary wood and protoxylem of the newly formed branchtips, which are in direct connection with the woody strands coming from the leaves. Hence even in the oldest trees there is a direct channel in the recently formed wood connecting the absorbing tips of the roots with the evaporating surfaces of the leaves.

\section{PRACTICAL WORK.}

Take the end of a branch of Pinus silvestris. Identify the long and dwarf shoots. Observe the circle of long shoots formed round the main stem at the base of each year's growth. Notice the subtending scale of the dwarf shoots, and their sheathing scales surrounding the bases of the two foliage-leaves. Make out the number of seasons during which the dwarf shoots persist. Record these observations in your sketches.

Prepare and mount thin transverse sections of a leaf. Make a sketch showing the epidermis with its thick walls and its cuticle, the sunken stomata, the inner fibrous layer, the mesophyll with its inflected cell-walls, the bifurcating cells beneath the stomata, the resin-ducts, the endodermis, the transfusion tissue, and the two conducting tracts formed of wood and bast.

Prepare and mount a transverse section of the terminal soft and succulent part of a branch, cut in early summer before the foliage leaves on the dwart shoots are mature. Sketch the section marking the epidermis, cortex, primary medullary rays and pith, The primary vascular bundles composed of protoxy- 
lem, primary wood, primary bast and protophloem should also be shown. The resin-ducts will also be conspicuous.

Prepare and mount transverse sections of a part of the stem which is two or three years old. Make an outline sketch of the whole. Fill in details on a sector of the sketch, showing the pith, the protoxylem, the primary wood, the primary medullary rays, the spring and summer wood of the two or three rings, the secondary medullary rays, the cambium, the secondary bast, the primary bast (the protophloem cannot be recognised) and the secondary cortex.

Transverse sections of the young growing tip of the root and of part which is two or three years old should also be prepared. Notice the change of the prismatic, or grooved, core of primary wood into a solid cylinder by the addition of new wood. The position of the protoxylem at the angles of the primary wood may be easily identified by the well-marked primary medullary rays which are directed radially inwards from the cortex, and terminate at the protoxylem. 


\section{LECTURE XXIII.}

WhILE the procambial tracts are forming the protoxylem tubes, the cells of the apex surrounding them are expanding rapidly. Large vacuoles develop in their protoplasm, increasing their volume at a quick rate. This expansion is chiefly in a longitudinal direction, and is probably to be attributed to the action of the osmotic pressure of soluble substances in the protoplasm in pushing out the plastic vertical walls of the cells. The water supplied by the wood tubes leading up to the apex allows this osmotic pressure to act. The elongation of these cells involves that of the protoxylem tubes, and the longitudinal extension of their walls is plainly indicated by the drawing out of their spiral thickenings and the increased spacing of the rings. The most rapid elongation of the apex takes place immediately after the differentiation of the first protoxylem tubes. Consequently those which are nearest the pith have the most widely spaced thickenings : those outside them, being formed subsequently to the period of maximum growth, have their spiral and annular thickenings closer together.

The protoxylem tracheids being formed in the midst of soft undifferentiated tissues are circular in cross section. Once secondary developments have consolidated the woody cylinder the new tracheids are developed under mutual pressure between the rigid woody cylinder and the cohering cortex outside. The effect of this pressure is seen in the fact that the secondary tracheids are prismatic in form-polygonal in cross section.

The development of these tracheids takes place somewhat as follows: each cambium cell is prismatic in form. Its long axis is parallel to that of the stem. Its cross section is rectangular and oblong and so oriented in the stem that its two longer sides are parallel to the surface (tangential) while the two shorter sides seen in the transverse section are radially disposed. These prismatic cells are terminated above and below by a bevelled end. If you have followed this description you will realise that the cambium cells appear as small rectangles in transverse sections, as very long rectangles in radial sections and as long parallelograms with sloping 
end walls in tangential sections of the stem. These cells increase in thickness in a radial direction, and after their nucleus has divided a longitudinal wall is formed which divides the prism tangentially into an inner and outer portion. The outer remains undifferentiated and grows and divides, the inner one slowly changes and becomes a tracheid. It elongates considerably, the originally bevelled end becoming more tapered and pushing between the similar ends of other developing tracheids above and below. During this growth the mutual pressure of these ends distorts their original form and forces them to become polygonal prisms. This distortion does not take place in the middle region of the developing tracheid, and consequently it retains its rectangular shape undistorted. Simultaneously with this elongation the developing tracheid grows in width. Its growth in width appears to be largely controlled by the water supply. When this is limited the osmotic pressure is unable to distend the wall. Hence the tracheids formed in periods of drought (usually in summer) have smaller cavities than those produced when water is abundant in the stem (viz. in spring). When mature the tracheids are about $2 \mathrm{~mm}$. in length and vary from $0.04 \mathrm{~mm}$. to $0.02 \mathrm{~mm}$. in width. While this enlargement of the cell has been taking place the protoplasm within has been active in adding to the thickness of the wall. The new layers composed of woody cellulose soon so increase the strength of the wall that further enlargement is impossible. Certain areas on the radial walls are not thus thickened but remain as thin circular patches on the wall of the tracheid. These patches are called pits. They resemble flexible membranes stretched across circular openings in the radial walls between adjacent tracheids. The central part of the membrane is slightly thickened and forms a cushion of cellulose called the torus : the peripheral part is excessively thin. On the margin of the pit the protoplasm forms a deposit of lignified cellulose which is at first a collar and then grows up into a dome overarching the pit membrane. The dome is never completed but leaves a more or less constricted opening in its top leading into the pit. Domes overarching the pit membrane separating adjacent tracheids are simultaneously developed. These structures on the walls are called bordered pits. They are completed in the cell before the remains of the protoplasm disappear and leave the tracheid without granular contents. The bordered pits are about $0.02 \mathrm{~mm}$. in diameter: they are placed in a series along the radial walls of the tracheids, 20 to 50 on each wall. Occasionally, especially in the summer tracheids, bordered pits are found on the tangential walls, The 
flexibility of the membrane allows it to be displaced, so that it may lie against the dome on either side, while the torus blocks the aperture of the dome. As will be explained in a later lecture, these peculiar little valves play an essential rôle in enabling the evaporating leaves to draw up a continuous supply of water through the plants.

A comparison of the appearances presented by the various structures composing the wood in sections taken in different directions will bring out clearly their relations and will enable you to reconstruct mentally the solid form of each. For this purpose it is necessary to examine the appearance of the tracheids, medullary rays, and bordered pits in three sections taken at right-angles to each other, viz. in transverse, radial and tangential sections.
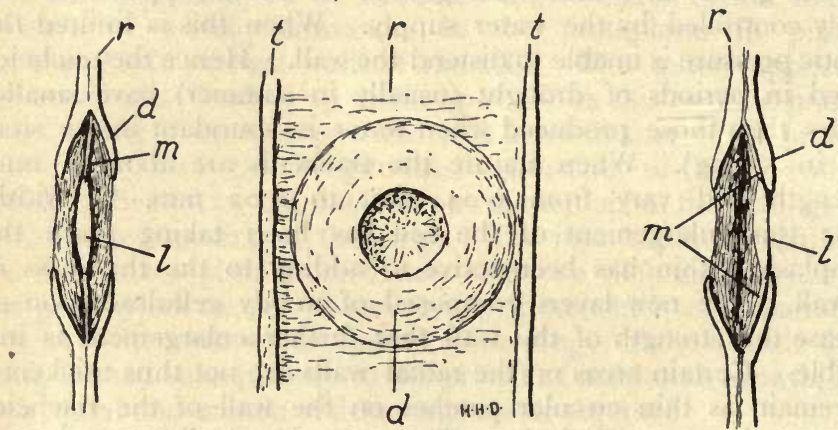

Fig. 58. - Pinus silvestris, plan (in middle), section (at either side) of bordered pit, $\times$ about rooo. $d$, dome ; $l$, torus ; $m$, membrane ; $r$, radial wall of tracheid; $t$, tangential wall. The section on the left shows the membrane and torus in a median position; that on the right shows them drawn against the dome.

\section{(I) Transverse section :-}

The whole of the secondary wood of Pinus, i.e. all the wood except an insignificant portion immediately round the pith, is built up of tracheids. Each cambial cell originates a radial row of them so that in transverse section we see their rectangular cross sections forming radial series. The regularity of these series is only disturbed if the section is at the level of the overlapping ends, or if, as occasionally happens, the cambial cell divides radially, and the radial series becomes double. As you have already learned the walls of the summer tracheids are thicker than those formed in the spring. In transverse sections these thick-walled tracheids form dark bands crossing the radial rows at right angles. These 
dark bands are separated from one another by light ones formed by the thin-walled spring tracheids. Comparison of the abruptness of the transition on either side of the bands will enable you to determine in which direction the pith and the cortex lie. In the transverse section, the pits are seen almost exclusively on the radial walls. At the level of a pit this wall in section looks like two Ys placed so that the ends of the two branches of one are approximated to the ends of the two branches of the other and connected across the angle of the $\mathrm{Ys}$ by a thin line. The branches of the

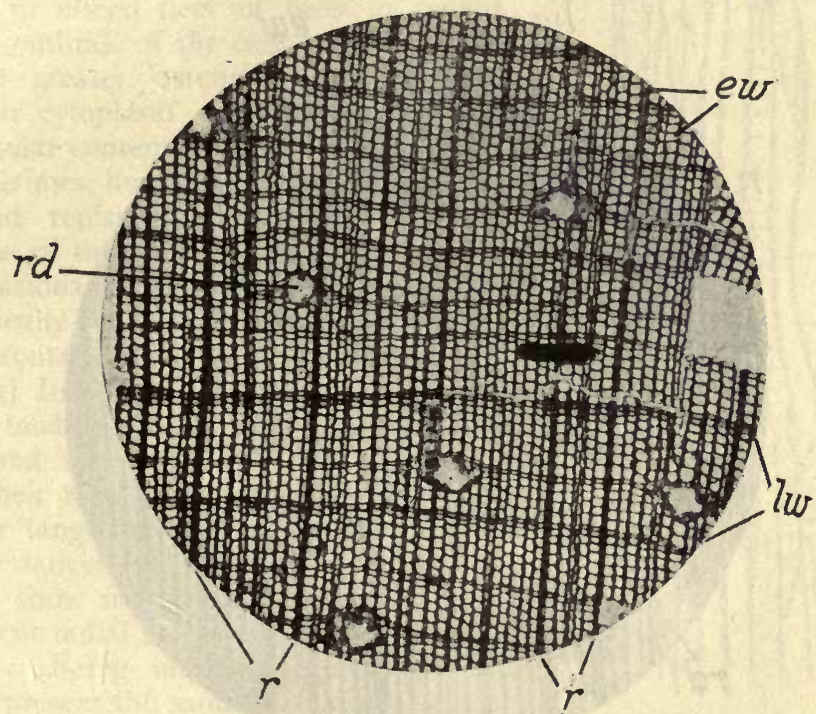

FiG. 59.-Pinus silvestris, microphotograph of transverse section of wood, $\times 32$. ew, early, or spring wood; lw, late, or summer wood; $r$, medullary ray; $r d$, resin-duct.

Ys represent the section of the domes on each side of the pit. The vertical limb of the Ys corresponds to the section of the radial wall.

In the transverse section of the wood the medullary rays are also seen. They appear as radial bands of cells, one cell thick, interposed between the radial rows of tracheids. The medullary-ray-cells appear oblong, their greatest extension being in a radial direction. Usually they are filled with protoplasm in which is seen a nucleus and often starch grains and oil globules. Sometimes the protoplasm has disappeared and the wall of the cell alone 
remains as a tracheid. Resin-ducts are also seen in the transverse section of the wood. They are found in thickened parts of the rays and may be cut transversely or longitudinally and are lined with a layer of cells which secrete resin into the ducts.

(2) A radial section of the wood shows the same structures as the transverse, but naturally they appear very different, being viewed from an aspect at right angles to that shown in the transverse section. The tracheids are seen in longitudinal section, their tangential walls being cut, while their radial walls are seen in

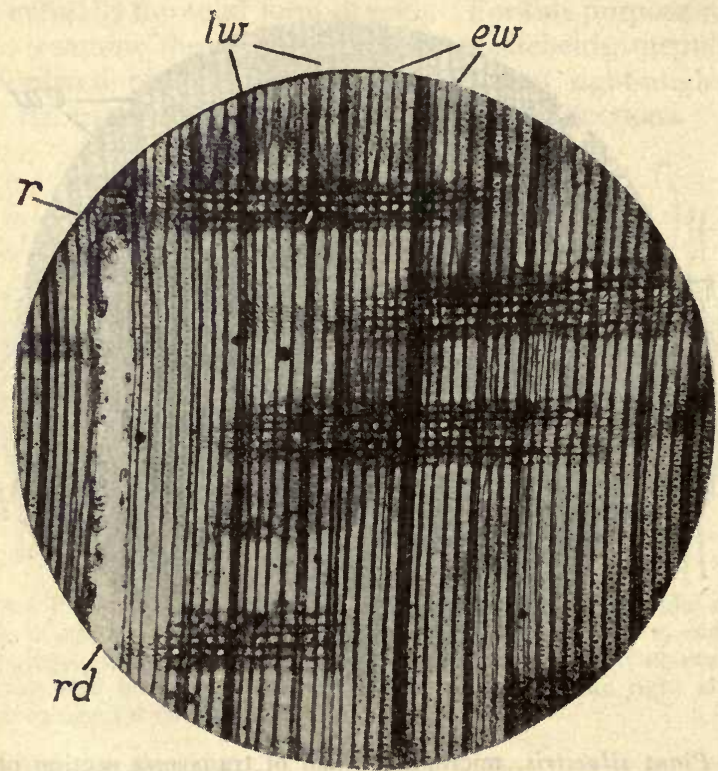

FIG. 6o. - Pinus silvestris, microphotograph of radial section of wood, $\times 32$. ew, early, or spring wood; lw, late, or summer wood; $r$, medullary ray; $r d$, resin-duct.

plan. In the summer wood the tangential walls are closer together than in the spring wood, consequently the wood tissue in that region appears more dense and the successive layers of summer wood appear as successive vertical bands alternating with the more transparent bands of spring wood. It is on the radial walls of the spring wood, between the more widely spaced tangential walls, that the bordered pits are most plainly seen. Focussing down upon them their structures come into view in the following suc- 
cession: (a) The opening of the dome, which is more or less slit-like; (b) the circular outline of the pit membrane within which is a smaller concentric circle, the outline of the torus; $(c)$ the opening of the dome on the further side of the membrane. In this radial section the medullary rays appear as walls of cells crossing the tracheids, varying from one to fifteen tiers of cells in height. The outlines of the cells are oblong and their greater extension is horizontal. Their cytoplasm with its nucleus and granular contents appear to fill the cells; sometimes, however, empty tracheids are found replacing these cells, especially those of the uppermost and lowest tiers. Occasional resin-ducts are seen rising vertically from ray to ray, or running horizontally in a ray.

(3) In tangential sections of the stem the tracheids are again seen in longitudinal section. This time, however, it is their radial walls which are cut and their tangential walls are seen in plan. The tangential walls are usually smooth and show no special structures, but on the cut radial walls are seen the bordered pits. Being radially symmetrical the pits present the same appearance on this section as they do on the transverse section. In tangential sections of the stem the medullary rays are cut at right angles to their course. Being walls of one cell thick and of a variable height they appear as vertical series of cells. The height is somewhat greater than their width. Most of them are filled with protoplasmic and granular contents, and often seem to bulge into the empty cavities of the adjacent tracheids. Often the uppermost and lowest tiers of cells have lost their contents and have

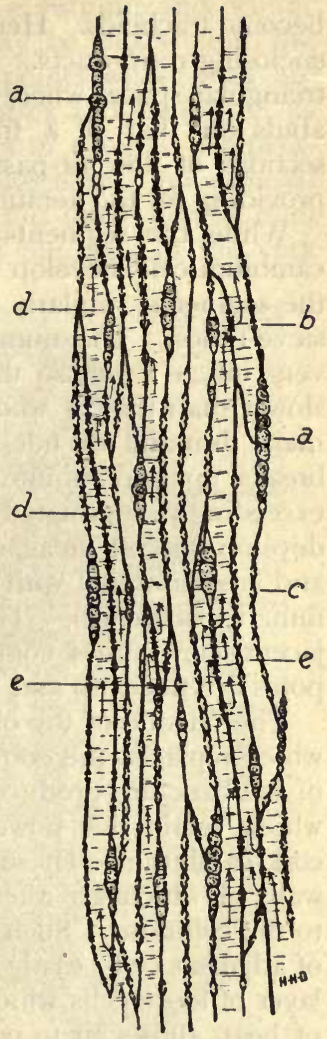

FIG. 6r. - Pinus silvestris, diagrammatic tangential section of wood transmitting transpiration-current, $\times 60$. $a$, medullary ray; $b$, bordered pit with membrane in median position and capable of transmitting water; $c$, bordered pit with membrane drawn against dome and capable of resisting tension of sap; $d$, tracheid full of water and transmitting current; $e$, tracheid emptied of water showing the bordered pits on its walls with membranes drawn against the domes. 
become tracheids. Here and there rays are seen several cells thick enclosing resin-ducts. Careful examination will also show minute triangular spaces where the wall separating two medullary-ray-cells abuts on that of a tracheid. These spaces represent the cross sections of fine air-passages running inwards along the rays and providing for the aeration of the inner tissues.

While the segments which are cut off the inner faces of the cambium cells develop in the manner just described into tracheids, the segments similarly separated from the outer faces grow into sieve-tubes. The number of segments formed on the outside is very much fewer, so that the rate of formation of bast is much slower than that of wood. The walls of these outer segments remain thin and do not become lignified. The nucleus of the cell breaks up into minute fragments and the cytoplasm becomes an exceedingly attenuated layer against the walls. The radial walls develop special areas, sieve plates, which are rounded in outline and are perforated with numbers of very fine pores (say $\mathrm{I} \mu$ or $0 \cdot 00 \mathrm{I}$ $\mathrm{mm}$. in diameter). Through these pores the protoplasm of adjacent sieve-tubes communicates. As the sieve-tube ages these pores are filled up and the communication is cut off.

The outside of the older parts of the stem is covered with bark which replaces the cortex of the younger parts. The outer parts of the bark are produced by a layer of cells situated in the cortex which retain the power of dividing. This layer is called the cork-cambium. These cells give rise to cells on their outside, the walls of which are altered by the addition of a substance, suberin, to the cellulose. Such walls are very impervious to water and those of adjacent cells easily part asunder. They thus form a covering layer of loose cells which, though waterproof and a bad conductor of heat, allows air to penetrate very slowly. It is called the cork. As the growth of the wood and bast stretches the cortex new corkcambiums are formed more deeply within the stem, while the outer cork spalls off as rough scales from the surface.

We have seen that there is direct connection between the wood of the young absorbing roots and that of the transpiring leaves through the outer layers of the wood of root and stem. Even in the lower parts of those stems where the wood is formed of the growth of perhaps many decades of years, by far the greater part of the upward water current is conveyed in a few outer layers. The older more axial wood acts as a skeleton round which the functioning tissues form a veneer. The carbohydrates and proteins formed in the leaves are usually supposed to pass down the bast to be distributed to the cambium and the growing regions of the branches 
and roots. Some are also stored during the autumn and winter in the medullary rays and next season may be thrown into the tracheids to be transmitted to the various points where they are needed for growth. The outer tissues-cortex and bark-act as non-conductors to diminish the suddenness of external temperature changes, as waterproofing to isolate the bast from external osmotic actions, and as armour to protect the inner tissues from injury. At the same time the looseness of the cells in the cork permits of an exchange of gases through this coating which is necessary for the respiration of the inner cells, viz. those of the cambium and medullary rays.

From this brief study of the vegetative structure of the Pine and cursory consideration of the function of its parts we must turn our attention to its reproduction.

Two kinds of cone are found on the Pine which are concerned in its reproduction : the dust- or pollen-cones and the seedcones. They are both borne near to the ends of the branches. We will begin with the examination of the pollen-cones.

The pollen-cones are yellow in colour, they are somewhat egg-shaped and are about $\mathbf{I} \mathrm{cm}$. long. They are attached to the

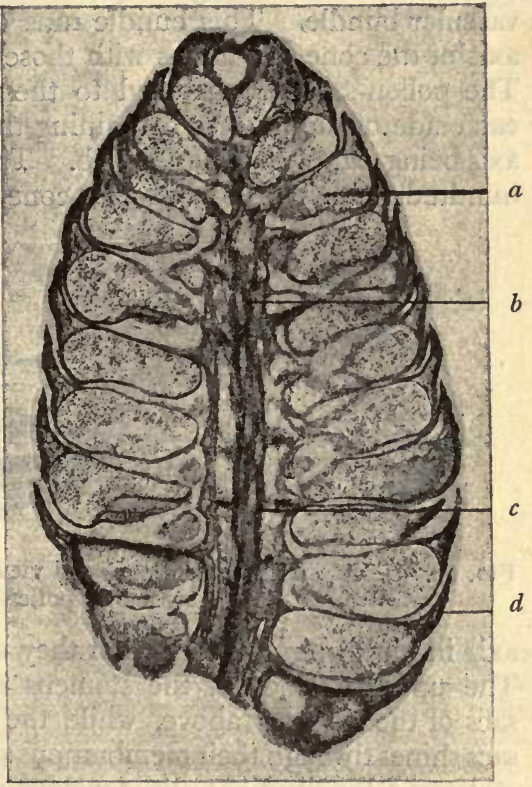

FIG. 62.-Pinus silvestris, pollen-cone, longitudinal section, $\times$ r2. $a$, pollen-sac; $b$, axis of cone ; $c$, conducting tract in axis; $d$, outer end of stamen. (From Evans' $A u$ Intermediate Textbook of Botany.)

present year's growth by short stalks. A large number of them form groups on the base of the long shoot replacing lateral dwarf shoots.

The stalk of the pollen-cone springs from the axil of a scale on the long shoot. The stalk itself bears a number of overlapping membranous scales, and is continued upwards as the thick, almost 
fleshy axis of the cone. On this axis above the membranous scales just noted, the sacs containing the dust or pollen are crowded. These sacs are roughly cylindrical with rounded ends; they are fixed in pairs on the under side of special triangular scales called stamens. The base of the stamen-the apex of the triangle-is attached to the axis, while the broader distal end of the stamen beyond the pollen-sacs is reflected upwards as a membranous flap. The greater part of the stamen is thin and membranous but down its middle line is a ridge of tissue which is traversed by a single vascular bundle. This bundle runs down into the bundles of the axis of the cone and unites with those of the supporting long shoot. The pollen-sacs are attached to the under side of the stamen at each side of the mid-rib containing the vascular bundle, their long axis being parallel to the mid-rib. There are between sixty and one hundred stamens in each pollen-cone and they are arranged on the
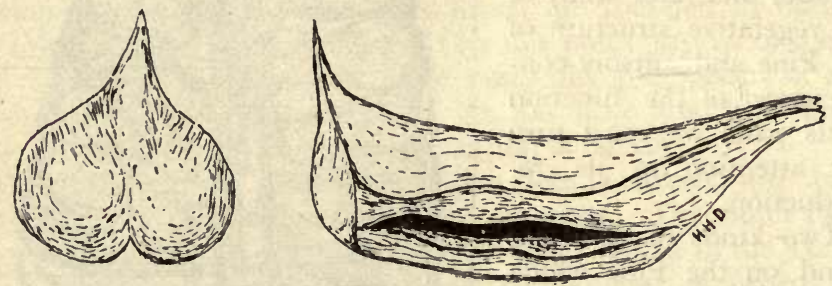

FIG. 63.-Pinus silvestris, stamen, end view on left, side view on right, $\times 25$. Side view shows pollen-sac dehiscing.

axis in close spiral lines so that they completely cover it from view. The upturned ends of the stamens cover the ends of the pollensacs of the stamens above, while the yellow colour of the pollensacs shines through their membranous substance. As the pollen-cone matures a longitudinal slit develops in the wall of each pollen-sac. This slit is formed by the contraction of the outer layer of cells in the wall which, as water evaporates from them, are compelled by their rigid internal supports to contract tangentially thus tearing the wall longitudinally. Through this rupture the yellow dustthe pollen-contained in the sac is released and falls on to the upper surface of the stamens below. The pollen is retained there by the upturned outer ends of these stamens. It is finally set free, by the elongation of the axis separating the stamens, and is launched on the wind by the swaying of the branches.

The pollen coming from these cones is a uniform fine yellow powder. Each grain is a rounded mass about $0.04 \mathrm{~mm}$. in diameter. 
It is contained in a thick cell-wall. The outer layer of this wall is formed of cutin and bulging away from the inner layer forms two large bladder-like outgrowths on the wall of the rounded mass. These bladders are each about half the volume of the rounded mass which lies between them. They contain air, and by decreasing the specific gravity of the pollen-grain and greatly increasing its surface they cause it to be easily carried on the wind. The central mass is composed of one large rounded cell into which projects a hemispherical structure composed of a small disc-shaped cell, the stalk-cell, and a small plano-convex cell, the body-cell.
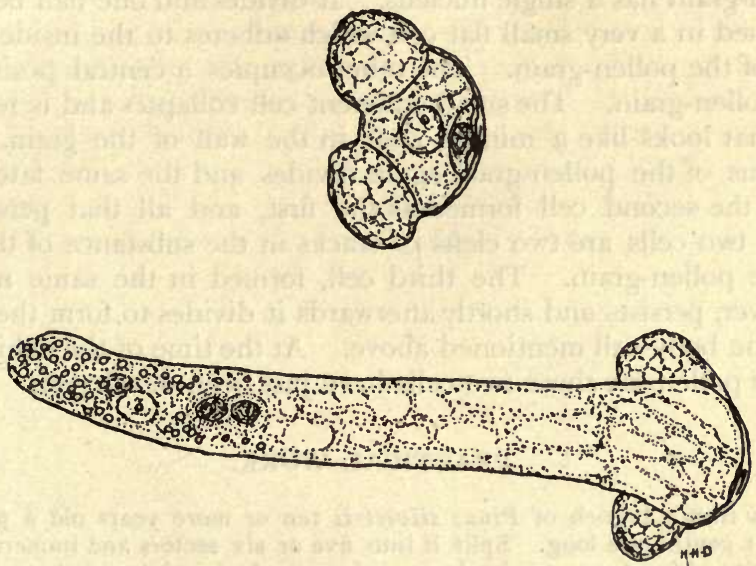

FIG. 64.-Pinus silvestris, pollen-grain, $\times 250$. That above, just about to germinate, shows two transitory cells against the inside of the coat of the pollen-grain. The lower grain has developed the pollen-tube. The two dark bodies in the tube are the nuclei of the sperm-cells, the two lighter are the nuclei of the tube and the stalk-cell respectively.

Acquaintance with the previous types suggests that the pollengrains are spores and that the sacs which contain them are sporangia, and if this is so, their relations to the stamens would indicate that these latter are spore-bearing leaves - sporophylls. A study of the development confirms these surmises. The stamens arise as excrescences on the side of the apex of the axis of a developing pollen-cone. They correspond with leaves in their development and in their relations to other parts. 'The pollen-sac arises on these leaves as a mass of cells. The cells forming some of the deeper layers of this mass differentiate as a group of thin-walled cells completely filled with protoplasm and having large nuclei. 
These are the pollen-mother-cells. The nucleus of each divides twice and produces four nuclei. Round each nucleus the cytoplasm segregates and forms a spherical cell. A cell-wall is formed covering each, and from the remains of the cytoplasm of the mother-cell the cutinised layer and bladders are formed. The four pollengrains arise as a tetrad in a mother-cell, in a manner quite similar to the spores of the liverworts like Marchantia, the mosses like Funaria and the ferns like Aspidium and Selaginella. Thus, considered from the point of view of development, the presumption that the pollen-grains are spores gains probability. At first the pollen-grain has a single nucleus. It divides and one half becomes enclosed in a very small flat cell which adheres to the inside of the wall of the pollen-grain. The other occupies a central position in the pollen-grain. The small adherent cell collapses and is reduced to what looks like a minute cleft in the wall of the grain. The nucleus of the pollen-grain again divides and the same fate overtakes the second cell formed as the first, and all that persists of these two cells are two clefts or cracks in the substance of the wall of the pollen-grain. The third cell, formed in the same manner however, persists and shortly afterwards it divides to form the stalkand the body-cell mentioned above. At the time of the dehiscence of the pollen-sac these two cells have just been developed.

\section{PRACTICAL WORK.}

Saw from a branch of Pinus silvestris ten or more years old a piece of about I centimetre long. Split it into five or six sectors and immerse them in spirit. After ten or twelve hours or longer, change the spirit for a mixture of spirit and glycerine, about half and half, and leave for twenty-four hours. This renders the wood more easy to cut. Split some of the sectors tangentially in the wood and some tangentially in the bast. Smooth all the cut surfaces with a sharp razor and prepare thin sections from the transverse, radial, and tangential surfaces. Place the sections in water and select some of the thinnest of them for staining. Lift these latter into a mixture of watery saffranine and Delafield's hæmatoxylin and stain for fifteen to thirty minutes. Wash in several changes of spirit and finally clear in oil of cloves. See that the sections remain completely submerged in the oil. When the sections appear quite clear-this may be tested by observing them in a strong light before a black background-lift them from the oil and lightly touch them against blotting-paper (to remove the free oil), and place them in a drop of canada balsam on a slide, and cover. During the staining of these sections mount and examine others in water.

Carefully examine the three kinds of sections, and sketch, using the high power, portions of the different tissues, viz. of the wood, bast, cortex and medullary rays, as seen in all three.

By careful comparison of the appearances presented in the three sections reconstruct a medullary-ray-cell and draw it in perspective, marking the length of each of its sides as determined by the Ghost-micrometer. 
Sketch, using the high power, the bordered pits as seen in the three sections.

Sketch a group of pollen-cones on the supporting branch. Using a simple lens, sketch one cone showing the scales and stamens. Detach a single stamen, and, using the low power or simple lens, sketch it from its upper and lower aspect, showing the pollen-sacs, the mid-rib, and upturned end.

Shake out some of the pollen on a dry slide and examine with a low power. Observe the central mass and observe its air-bladders. Shake out others into a drop of spirit, cover, examine and sketch. Note the large cell and the small enclosed group of the stalk- and body-cell. Careful examination will reveal one or two of the crack-like vestiges of the temporary cells. 


\section{LECTURE XXIV.}

OTHER cones are found on the pine which in contradistinction to the pollen-cones are called seed-cones. Like the former they are found among the dwarf shoots at the ends of the branches; but while the pollen-cones are found in groups replacing groups of dwarf shoots, the seed-cones occur in small numbers and are formed at the end of the annual growth replacing lateral shoots of unlimited growth. The pollen-cones persist only for a single season and then

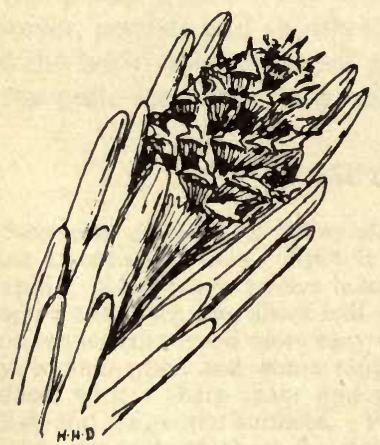

FIG. 65.-Pinus silvestris, young seed-cone surrounded by short shoots, $\times 4$.

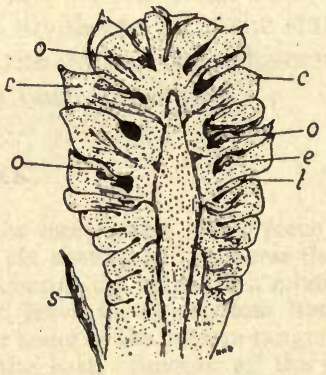

FIG. 66.-Pinus silvestris young seed-cone, longitudinal section, $\times$ 37. $c$, seed-scale (carpel); $e$, embryo-sac; $l$, bract-scale; $o$, ovule (megasporangium); $s$, scale.

fall off, after the pollen is shed, leaving a bare region on the stem, while the seed-cones remain attached for three seasons, and are to be seen in the first, second and third year of their development adhering to the same branch separated from one another by a season's growth. During this development their appearance changes profoundly. The young seed-cone is found beside the apex of a branch; it resembles a small green bud and is eggshaped. As it grows its green becomes tinged with crimson, and 
by June in the first year it is an egg-shaped body about half a centimetre long and about $0.25 \mathrm{~cm}$. in diameter. It is attached by a short stalk close to the apex of the supporting stem. The stalk is comparatively stout and is prolonged upwards as a succulent axis to the cone. On it are set some sixty or seventy small wedgelike scales arranged round the axis in a series of spirals. These scales are called carpels. They are at first of a succulent consistency. Closer examination of the carpels shows that each is composed of two parts, the large, fleshy wedge-like scale and a small spur-like projection springing from its base and united to its under surface. On the upper surface of the larger scale near its base and on each side of its middle line is a rounded or egg-shaped body, the ovule. Later on, this ovule becomes the seed and hence the fleshy scale is often called the seed-scale, while the small spur-like scale is termed the bract-scale. It may be mentioned that in some trees similar to the pine the bract-scales are relatively much larger, thus in the larch they project beyond the seed-scale and in the fir the seed-scale seems to be an excrescence upon the bract-scale. In fact the whole carpel might be compared to the leaf of Selaginella subtending a sporangium, the seed-scale corresponding to the ligule disproportionately enlarged.
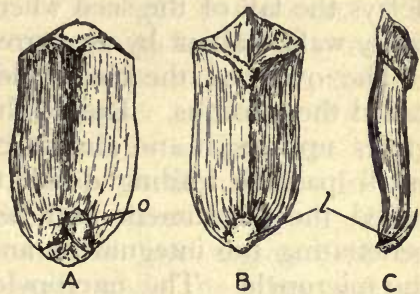

Fig. 67.-Pinus silvestris, seed-scale (carpel). A, seen from above; B, from below; and $\mathrm{C}$, from the side. $o$, ovules; $l$, bract-scale.

At the distal end of the preceding year's growth are found seedcones which are one year old ; they are some 4 or $5 \mathrm{cms}$. long ; they are green in colour and instead of standing out erect from the supporting branch their apex is turned downwards by the curvature of their stalk. The spaces between the carpels have been closed by the growth of the latter and now the outer ends of the carpels fit closely and cohere together, so that one sees only the outer ends of the seed-scales. They appear as quadrate areas on the surface of the cone and each is raised in its middle so as to resemble a low blunt pyramid. When the carpels of the cones in this stage of development are examined, their seed-scales are found to be much enlarged and fleshy though somewhat tough structures. The bract-scales on the other hand have not developed and are only found with difficulty, persisting as minute tongues or spurs at the inner end of the carpel. The ovules have much increased in size and a re easily visible as egg-shaped whitish masses 3 or $4 \mathrm{~mm}$. in 
length. Their outer ends seem embedded in the scale while their pointed inner ends project free from it and are directed towards the axis of the cone at the side of the attachment of the scale to the cone. This pointed end is often tipped with brown.

The cones which are two years old are found just below the growth of the preceding year. They are brown and woody, but have not grown further in size. Their stalk is straight and the scales are widely divergent from one another. By this time the ovules have become seeds and some of them are very probably already shed. The seeds are larger than the ovules and they are brown in colour. They are covered with a hard coat to which is attached a brownish semi-transparent wing formed from some of the tissue of the upper surface of the seed-scale which has adhered to the seed and detached itself from the seed-scale. This wing delays the fall of the seed when shed from the cone, and makes it easily wafted about by air-currents.

The ovule on the seed-scale consists of a small mass of tissue called the nucellus. Round this, a collar of tissue develops which grows up over it and almost completely covers it, leaving only a small passage leading down to the nucellus. The covering is called the integument and becomes the seed-coat; the passage penetrating the integument and leading to the nucellus is called the micropyle. The micropyle is directed towards the axis of the cone and slightly away from the middle line of the upper surface of the carpel. One of the cells centrally placed in the growing nucellus elongates parallel to the axis of the nucellus and divides transversely into four. One of these four grows rapidly, while it crushes and partially absorbs the remaining three and other surrounding cells. This enlarging cell is known as the embryo-sac. As we shall see, it is in reality a megaspore. The megaspore continues to enlarge so that its protoplasm is reduced to a lining adhering to the wall. Meantime its nucleus divides and this division is succeeded by divisions in its products. The numerous nuclei so formed are distributed in the layer of cytoplasm lying against the wall of the megaspore or embryo-sac. Cell-walls now begin to be formed and separate the superficial nuclei into cells. The unenclosed nuclei continue to divide and the cytoplasm increases in bulk, gradually invading the central vacuole. With the continuance of cell-division the whole cavity of the embryo-sac is ultimately filled with tissue, which is called endosperm. During this time the nucellus is considerably encroached upon and distorted by the growth of the embryo-sac. It is finally reduced to a conical cap of cells covering the top of the endosperm. The apex of this cap 
is immediately underneath the micropyle. Early in the second year of the cone the endosperm is thus an egg-shaped mass occupying the greater part of the ovule. About this time certain cells on the surface of the endosperm grow in size. These cells are about five or six in number and are situated round the pole of the endosperm which is nearest to the micropyle. These each divide into an inner and outer cell: from the former an ovum is developed and the latter produces the short neck of an archegonium. Evidently the endosperm is a gametophyte and the cell in which it develops is a megaspore quite comparable with that of Selaginella. The ovum grows rapidly in size, and the adjoining cells of the gametophyte differentiate as a capsule or venter enclosing the ovum, and convey to it the necessary materials for its growth. The cells surrounding the archegonium grow up round the neck, so in this way the latter finds itself at the bottom of a slight depression. The ovum, which by this time has grown so as to be about $\mathbf{I} \mathrm{mm}$. in length and is consequently easily visible to the naked eye, contains a large nucleus which is located in its upper end near the neck. This nucleus now divides just as the nucleus of the ovum of the archegoniates divides in preparation for fertilisation. In this division a small lenticular mass of protoplasm is separated immediately below the neck. It contains one segment of the nucleus and forms the ventral canal-cell, while the other segment persists as the nucleus of the mature ovum which now awaits fertilisation.

To understand how fertilisation is effected we must return to the pollen which has been shed from the pollen-cone. This dust is scattered abroad in great quantities so that some of it falls on, or is wafted to, the ends of all the branches in the neighbourhood of the pollen-cones. Some falls on the young seed-cones standing erect at the top of the current season's growth. The carpels of these are divergent and some of the pollen-grains find their way down between them to their base. Here they are detained by a drop of sticky fluid exuded from the micropyles of the immature ovules. As this fluid decreases by evaporation or absorption the pollen-grains are drawn by its retreating surface through the micropyle and are left adhering to the surface of the nucellus. The tube-cell of the pollen-grain now bulges out and presses into the top of the nucellus, firmly anchoring the grain there. The carpels of the cone close the spaces between each other by their growth and thus the pollen-grains are completely shut in from adverse conditions and remain quiescent during the autumn, winter and spring. 
In the spring and early summer the megaspore, as we have seen, germinates within the nucellus and becomes filled with the tissue of the gametophyte (prothallus, endosperm) and forms its archegonia. During the germination of the megaspore the pollen recommences its development. The tube-cell penetrates more deeply into the nucellus and the body-cell breaks away from the stalk-cell. Its cell-wall disappears and it divides to form two cells which pass into the hypha-like tube-cell growing through the nucellus towards the archegonia in the gametophyte. These two

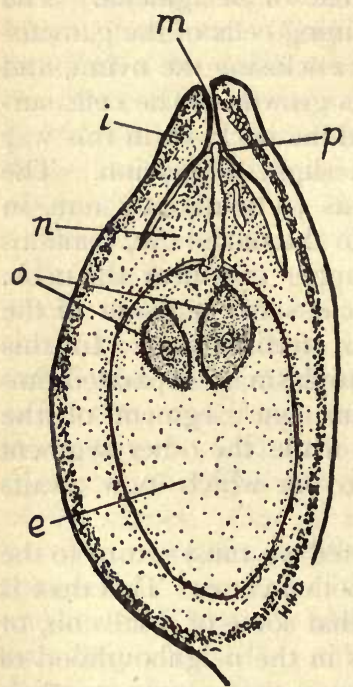

Fig. 68. - Pinus silvestris, ovule at time of fertilisation, longitudinal section, $\times \mathbf{I} 8$. $e$, endosperm ; $i$, integument ; $m$, micropyle; $n$, nucellus; $o$, ova; $p$, pollen-plant. cells, as their subsequent history shows, are the sperms or male gametes. They are conveyed in the pollen-tube to the neck of one of the archegonia and when the tube forces its way between the neck-cells, its tip becomes dissolved and the two sperms along with the other nuclei of the pollentube pass into the ovum. The nucleus of one of the sperms fuses with the nucleus of the ovum and fertilisation is effected.

Evidently we should regard the filiform growth from the pollen-grain as a male gametophyte which develops parasitically on the nucellus, and when mature produces the sperm-cell which fertilises the ovum. Thus the two gametophytes of the pine are very diminutive in size and parasitic in their nutrition. They both reach maturity one year after the germination of the spore.

The nucleus of the oosperm divides immediately into two and then into four. These four nuclei move to the basal end of the oosperm and become shut off from one another by cell-walls. The four cells so formed each gives rise to a filament of four cells. The cell of each filament furthest from the neck of the archegonium becomes an embryo and the remaining part of the filament elongates rapidly and is called the suspensor. It has the function of pushing the growing embryo more deeply into the surrounding gametophyte (endosperm) according as the food materials in that tissue become 
exhausted. In this way four embryos may be formed from the one oosperm : only one of these, however, continues its development. The others are absorbed along with the material of the endosperm by the successful one. In some other conifers the four cells below the suspensors cling together and jointly give rise to one embryo.

The embryo now develops into a cylindrical mass of cells, lying lengthwise in the ovule which is now becoming the seed. The blunt end of this embryo is turned away from the micropyle and becomes the growing region of its developing stem, and round it grows a crown of embryonic leaves-the cotyledons-about twelve in number. The other end of the embryo becomes the root, or radicle, and is covered by a large root-cap. Round the embryo and in close contact with it, is the gametophyte or endosperm, which has by this time absorbed most of the nucellus. The only remnant of this latter is a paper-like membrane intervening between the hard seed-coat and the gametophyte. In this condition the seed is shed from the seed-cone and by means of its wing is borne on the winds often to considerable distances.

After a period of quiescence the seed germinates if suitable conditions prevail. The embryo then recommences its growth and, absorbing the food material stored in the endosperm and rupturing the seed-coat, pushes out its radicle through the micropylar end of the seed. The stem below the cotyledons elongates while the cotyledons remain in the seed and draw out the supplies which it contains. Finally when all the stores are exhausted the seed-coat is shoved off and the cotyledons expand, and the seedling starts on its independent life.

The short study we have devoted to the life-history and structure of the Scots' Pine, insufficient though it be in many respects, leaves us in no doubt as to there being a general similarity between the tree and the comparatively small ferns Aspidium and Selaginella. The general manner in which they are constructed and the peculiar metamorphoses which they undergo are fundamentally similar in both cases.

An alternation of generations has come to light in the lifehistory of the pine. The fact that the gametophyte generation is wholly enclosed in the sporophyte renders this less evident, but the study of the origin and development of the pollen-grains and embryosacs establishes beyond doubt that the former are homologous to the microspores and the latter to the megaspores of Selaginella. Thus the Pine is heterosporous. Further, the pollen-tube and its contained cells are evidently homologous to the male gametophyte 
just as the endosperm contained in the megaspore or embryo-sac is homologous to the female gametophyte of Selaginella. The female gametophyte of the latter possesses chloroplasts and produces rhizoids and consequently is at least partly independent and self-supporting, but the male gametophyte is dependent for its supplies throughout its short life on the stores laid up by the sporophyte. Neither of the gametophytes of the pine show any

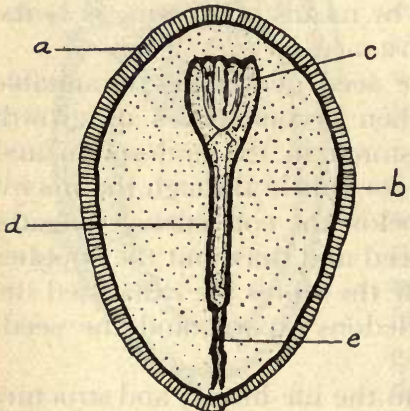

Fig. 69.-Pinus silvestris, seed longitudinal section, $\times 9 . \quad a$, seed-coat; $b$, endosperm; $c$, cotyledons; $d$, root; $e$, suspensor.

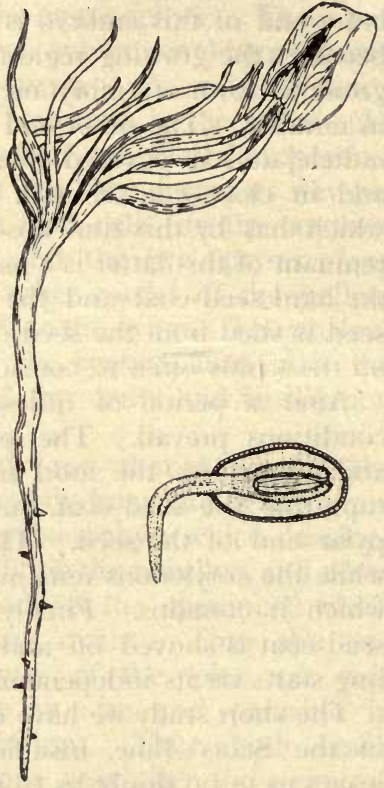

Fig. 70.-Pinus, germinating seed and seedling, $\times 3$. (After Sachs.)

independence of the sporophyte, but both, like the male gametophyte of Selaginella, are wholly dependent on the sporophyte for their food. The dependence even appears to go further, for both require in addition the nursing attention of the living cells of the parent sporophyte to administer the food to them. Their attitude towards the sporophyte may thus be compared to that of a child towards its nurse or of a parasite towards its host. Evidently while the sporophyte has gained in importance and complexity the gametophyte is reduced in size and independence. 
The increase in the proportional size and in the complexity of structure of the sporophyte has resulted in giving to the pine a vastly more efficient equipment for life on the land. The mechanical arrangement of its parts and the details of its structure, internal and external, enable it to carry on its life under the ordinary conditions on the land even when exposed to dry atmospheric conditions and a relatively small supply of water in the soil.

This complete equipment of the sporophyte for life on the land would be useless if, as in the case of the Fern and Mosses, the gametophyte required aquatic conditions for the passage of the sperm to the ovum. The ability of the male gametophyte to live as a parasite on the nucellus and the retention of the female gametophyte within the ovule enable this generation to dispense with aquatic conditions while the sperm cells are transmitted through the tissues of the sporophyte in the pollen-tube to the ovum. The possibility of this state of things is led up to, as we have seen, in a species of Selaginella where the microspores fall on to the megaspores before they are shed from the megasporangium, and the fertilisation of the female gametophyte is effected there. In the heterosporous Pine the connection of female gametophyte and the megasporangium is more complete and more prolonged. There the inner tissues of the ovule or megasporangium remain in nutritive contact with the megaspore (embryo-sac) throughout its germination, its development and sexual maturity. Even later during the development and early differentiation of the embryo this connection is maintained and is continued, till both the gametophyte and the tissues of the megasporangium are finally disintegrated by the germination of the seed. This retention of the female gametophyte in the megasporangium and the continued nutritive connection between the two till the independence of the embryo is established constitute the really important characteristics which distinguish Seed-Plants from the present ferns. It is a point of the highest interest that this distinction was not always maintained. Numbers of fossil heterosporous fern-like plants have been found having these characteristics. They belong to an ancient flora and date as far back in the Earth's history as Palæozoic time. These plants have died out in more recent periods, and have left a gap separating the present ferns and the Seed-Plants.

From another point of view the seed is a most remarkable structure. In its tissues are found an epitome of the whole lifehistory of the seed-plant. Its coat and remnants of the nucellus are portions of a sporophyte: the embryo-sac and endosperm 
represent the gametophyte; the embryo enclosed in these latter is the new sporophyte. In the seed, then, we have represented three generations; to these we must add the remnants of a fourth individual, the pollen-tube or male gametophyte, which persists

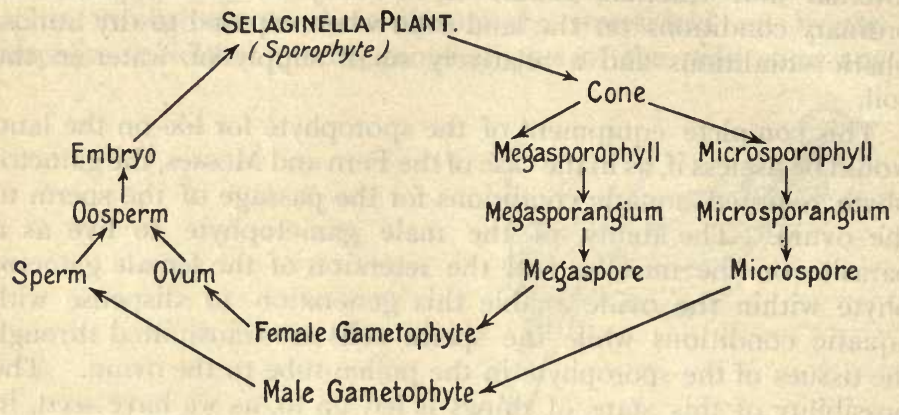

FIG. 7r.-Diagram summarising the life-history of Selaginella.

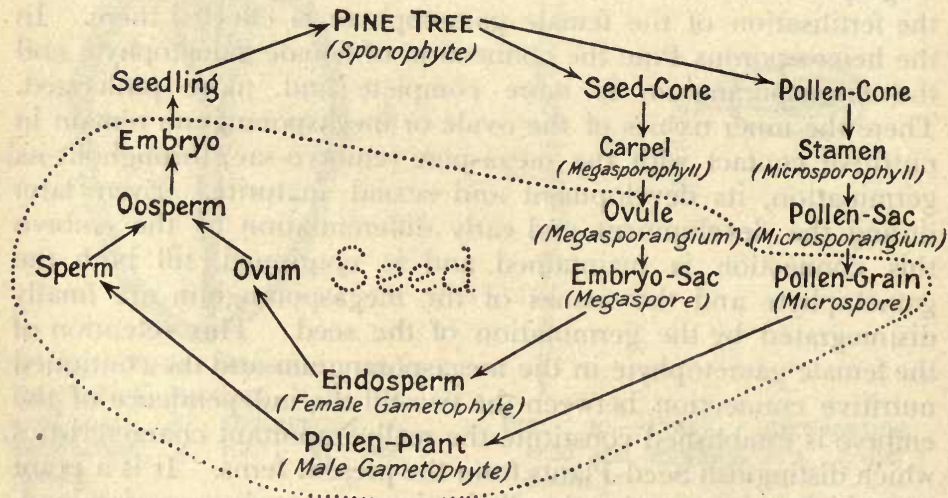

FIG. 72.-Diagram summarising the life-history of the Pine.

in the nucellus after fertilisation. It is a megasporangium containing a megaspore, a female gametophyte, an embryo-sporophyte, and the remnants of a microspore and male gametophyte.

I will now chalk upon the blackboard a diagrammatic scheme summarising the life-histories of Selaginella and the Pine Tree, which you will find helpful in understanding the homologies and relations of their various parts. 
The heterospory of Selaginella produces a slight differentiation in the later stages of the sporangia. The four large megaspores distort the shape of the containing sporangium, so that as the spores come to maturity it is distinguishable from the smaller and smoother microsporangium. The differentiation of the sporangia is much more complete in the pine, and there is little similarity in form between the microsporangia or pollen-sacs and the megasporangia or ovules. Furthermore, the leaves with which the two kinds of sporangia are associated are widely different in form and are easily distinguished as carpels or megasporophylls and stamens or microsporophylls. As in Selaginella the sporophylls are grouped together on axes to form cones or strobili but, while in Selaginella the microsporophylls and megasporophylls occur together in the same strobilus, in the Scots Pine they are separate and form microstrobili (pollen-cones) and mega-strobili (seed-cones). In other pines these two kinds of strobili occur on different trees or sporophytes, and thus there is a differentiation into microsporophytes and megasporophytes.

Thus we may trace in the Archegoniates and Seed-Plants a series beginning with the simple differentiation of the sexual cells formed on the same gametophyte as is found in Aspidium, passing to the differentiation of not only the gametes but also of the gametophytes, and finally leading up to those cases where two kinds of sporophytes are developed as well as two kinds of gametophytes.

The comparison of the individuals belonging to these different groups, the tracing of their life-histories and the establishment of the various homologues exhibited by each has contributed much to the philosophy of botany, and has controlled speculation by exact knowledge.

The occurrence of the alternation of generations in so many different groups of plants, its modifications and the extraordinary gradations connecting these modifications with one another constitute a problem which ever and anon obtrudes itself on the reflecting mind. Why should the life-histories of the different groups of plants resemble each other in their broad outlines? Why should intermediate forms be found connecting the most diverging extremes? What is the underlying principle responsible for the existence of homologues?

Although we are still far from a complete answer to these questions, the endeavour to find a solution for them has contributed, as we shall see later, some of the most elevating speculations of biological science. 


\section{PRACTICAL WORK.}

Sketch the end of a branch of Pinus silvestris, cut in June of three seasons' growth, and with seed-cones attached to the terminal portions of each increment.

Cut a green cone of the preceding season down its axis with a sharp knife. Sketch the smooth cut surface, showing the axis, the seed- and bractscales, and the ovules.

Isolate a single carpel and draw it from its upper and its lower aspect, showing the point of attachment, the two ovules on the upper surface, and the minute bract-scale on the under surface.

Make several median longitudinal sections of the ovules passing through the micropyle. From a comparison of the clearest make out the position of the integument, nucellus, female gametophyte (endosperm), and archegonia with their large ova. Notice also the inroads of the male gametophytes into the pointed end of the nucellus and the downward passage of pollentubes towards the archegonia. Record these observations in drawings. Estimate the actual size of the gametophytes and the ovum by means of the Ghost-micrometer.' 


\section{LECTURE XXV.}

SEED-PLAnTs are divided into two great subdivisions. The first comprises plants resembling the pine, and they bear their megasporangia or ovules quite exposed so that the microspores may be carried directly to the nucellus. In the second subdivision the ovules are completely enclosed in a capsule formed by a single megasporophyll (carpel) or by several megasporophylls cohering together. In this case the pollen-grain itself does not reach the ovule but is arrested by a special receptive portion of the carpel. There it germinates and produces a tubular gametophyte for traversing the tissue of the carpel as well as that of the nucellus. The first subdivision is called the Gymnosperms; the second, the Angiosperms.

To illustrate the salient features of these Angiosperms we select two examples. The first of these is the Buttercup. Any kind of Buttercup will serve our purpose, but perhaps Ranunculus bulbosus or Ranunculus repens are the most easily obtained.

The plant is herbaceous and its leaves, stems and roots are easily recognised. In the case of $R$. bulbosus the upright stems spring from a bulbous mass from the lower side of which many cord-like roots grow. The larger green leaves, which are comparatively soft and hairy, are attached to the top of the bulb by their somewhat hairy gutter-shaped stalks. The upright stems bear smaller leaves with short widened stalks; their branches terminate in flowers. The leaves are subdivided into three leaflets (pinnæ) and these are further so deeply indented as to form leaflets of a second order (pinnules). The leaf-stalk is continued upward and forms a rachis connecting the pinnæ together, and ribs on the pinnæ and pinnules run down and pass into the rachis.

The upright stems are tapering cylinders. They are hollow and tubular, as may be readily seen by cutting them across. The wall of these tubular stems is a few millimetres thick, and is composed of the fundamental tissue and of the vascular bundles. When cut across the fundamental tissue appears a translucent green, slightly darker towards the outside. Embedded in this are 
whitish cords much tougher than the fundamental tissue. These are the conducting tracts or vascular bundles. It is possible to isolate one of these cords and pull it out of the softer fundamental

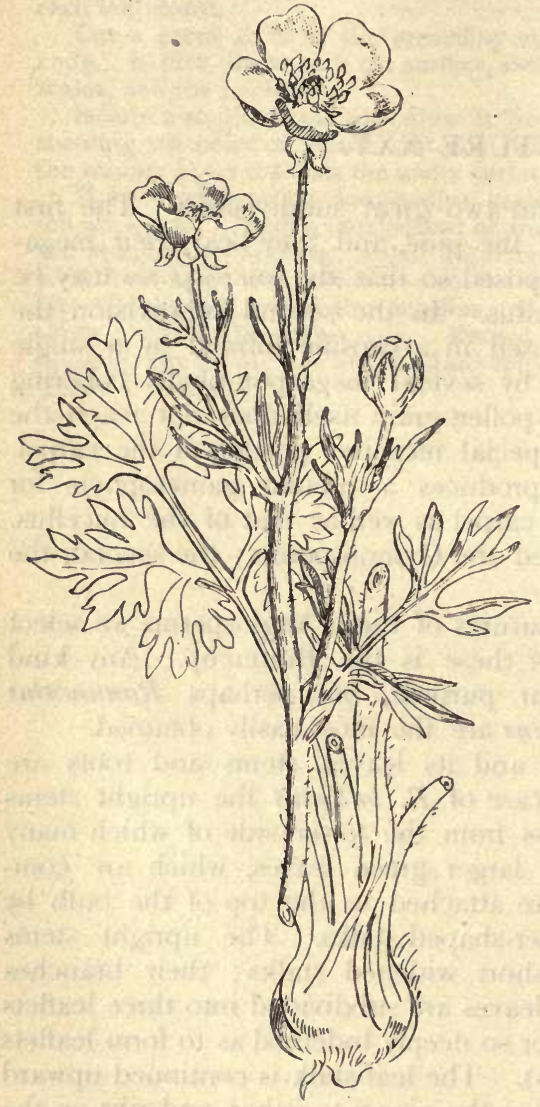

Fig. 73.-Ranunculus bulbosus. (After Sowerby.) tissue and trace it upwards or downwards in the stem.

Microscopic investigation reveals the finer details of structure. Here as in the ends of the branches of the Pine the fundamental tissue is divisible into cortex, medullary rays and pith. The surface of the stem is formed by an epidermis, one layer of cells thick. The outer walls of these cells are coated over with thin cuticle, which forms a more or less waterproof and impermeable membrane over the whole stem. The cuticle and the epidermis are perforated here and there with stomata, each guarded by a pair of cells. These guard-cells are the only cells of the epidermis which possess chloroplasts. The other cells of the epidermis are colourless. Usually one can see a nucleus and a lining film of cytoplasm in each. Here and there isolated cells of the epidermis grow out at right-angles to the surface as tapering spinelike hairs. Within the epidermis is the cortex. It is formed of thin-walled cylindrical cells, with rounded ends, set parallel to the axis of the stem. These cells contain the usual cytoplasmic lining in which are embedded a nucleus and many chloroplasts. A large vacuole occupies the middle of the cell. Between these cells are 
conspicuous intercellular spaces, which are more striking in the neighbourhood of the stomata. The cells of the deeper layers of the cortex are poorer in chloroplasts, and close to the connecting tracts its cells possess few or none; but they often contain numbers of starch granules. The cells of the medullary rays and pith are similar to those of the inner cortex, and sometimes contain even larger amounts of starch. The cells of the pith extend for only a few layers inside the conducting tracts, the whole axial region of the stem being occupied by the central cavity.

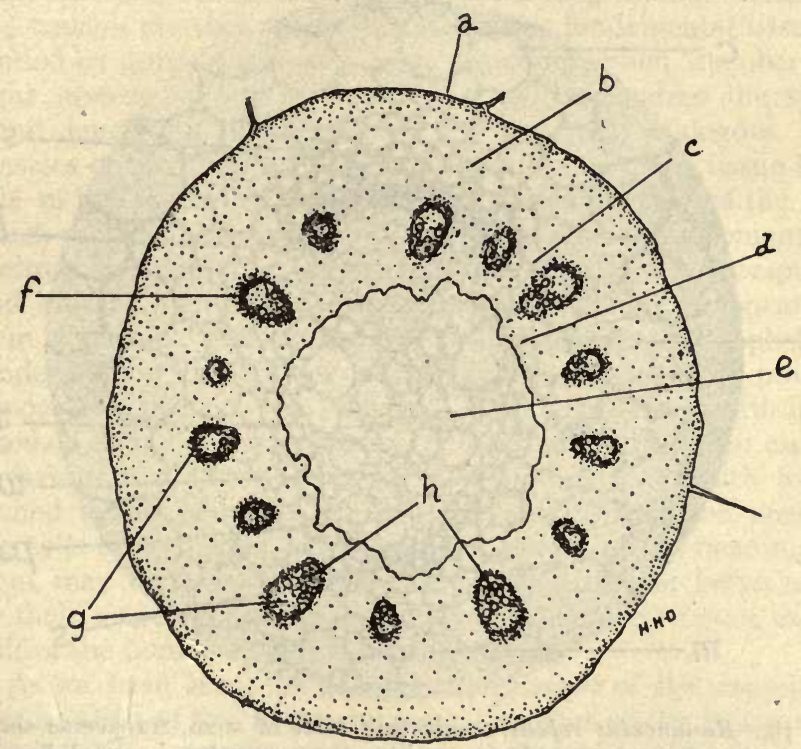

Fig. 74. -Ranunculus repens, stem, transverse section, $x$ ro. $a$, epidermis; $b$, cortex ; $c$, medullary ray; $d$, pith; $e$, space in pith ; $f$, bundle-sheath ; $g$, bast; $h$, wood.

The conducting tracts or vascular bundles form very distinct cords of tissue traversing the fundamental tissue in a longitudinal direction. They are formed like those in the young Pine stem of two parallel strands-the wood (xylem) and the bast (phloem). These tracts stand out more from the surrounding tissues than do those of the young Pine stem. This is chiefly due to the fact that each tract is enclosed in a tubular sheath of tough and fibrous tissue-the bundle-sheath. But the most important difference 
between these conducting tracts and those of the Pine is that they do not possess a layer of cambium between the bast and the wood, and hence these bundles do not grow in thickness. The dividing cells of the procambial tracts differentiate completely into wood, bast and fibrous bundle sheath leaving none over to develop as cambium. Bundles or conducting tracts which like these have no cambium are called "closed bundles" in contradistinction to those which continue to grow in thickness, which are called "open".

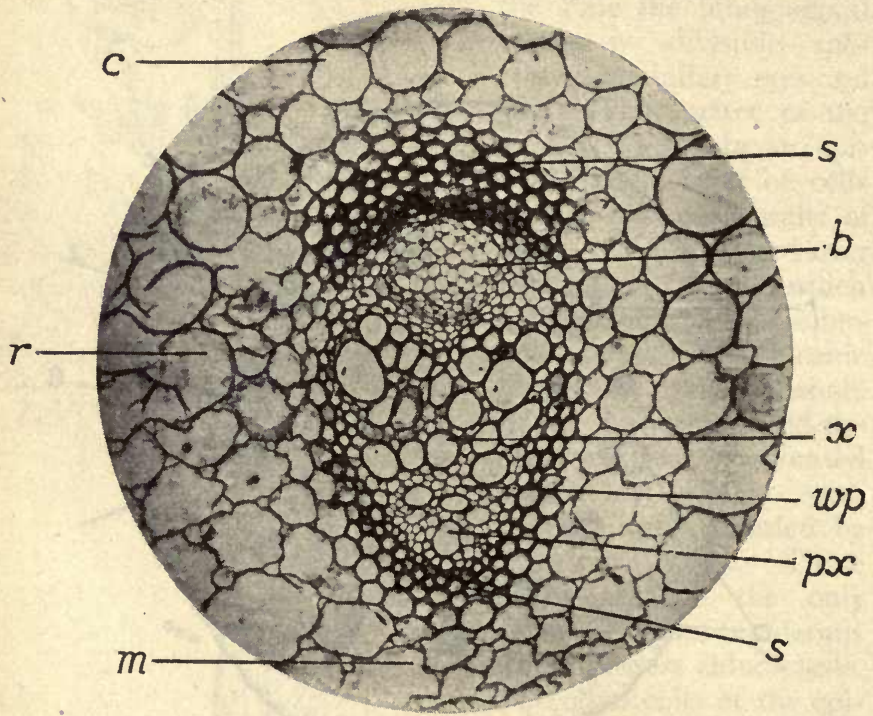

Fig. 75.-Ranunculus repens, conducting tract of stem, transverse section, $x$ roo. $b$, bast; $c$, cortex : $m$, pith ; $p x$, protoxylem; $r$, medullary ray; $s$, sheath; $w p$, wood-parenchyma; $x$, tracheæ of wood.

In general it will be found that trees and shrubs have "open" bundles in their stems and roots, while plants with "closed" bundles remain herbs. The vascular bundles of practically all leaves are "closed".

The bundle-sheaths appear to discharge the functions of a skeleton, i.e. they support and protect the soft tissues; but their exact rôle in these functions is not perhaps at first sight obvious. The cells forming the sheath are long narrow spindle-shaped cells. Their walls are very thick, so that the cavity of the cells is greatly 
reduced. They fit together closely and their tapering ends overlap one another for a confiderable distance. The total cross section of the ensheathing tube enclosing the conducting tract is small. Its greatest diameter does not exceed $0.5 \mathrm{~mm}$. Hence, by its own stiffness it contributes little to the rigidity of the stem. Its stiffness is further lessened by the fact that it is always extremely reduced in thickness and often even discontinuous along two lines, one at each side of the conducting tract. The fact is the sheath is not constructed to oppose transverse stresses, under which it is quite flexible, but to take up and resist longitudinal stresses. It is a tension member, while the cells of the fundamental tissue, distended by osmotic pressure, form the compression member of the rigid system. Thus in the rigidity which enables the stem to stand erect the bundle-sheaths play a part analogous to the muscles of the himan body, while the fundamental tissue has the rôle of the skeleton. The case is parallel to that of the leaves, which we have already noticed, and may be similarly demonstrated. Destroy the resistance of the fundamental tissue to compression, and without altering the mechanical properties of the sheaths, the stem collapses. As we have seen, this may be accomplished by rendering the protoplasm of the cells permeable by poisonous vapours or by heat. A protective function of the sheath is exercised on behalf of the strands of the conducting tract and especially in favour of the bast. The outer section of the sheath forms an arched tunnel in which the bast is protected from the pressure of the cells of the cortex, which at times, owing to the bending of the stem, may be considerable. The woody tubes are better adapted by their own structure to resist this pressure, and hence the inner half of the bundle-sheath is less developed.

As we have seen, the general arrangement of the tissues in the mature stem of the Buttercup resembles that of the young stem of Pinus. Changes take place subsequently which make the structure of the older stem of the Pine more complicated. Hence, we might expect the tissues of the strands of the conducting tracts would be less complicated than those of Pinus. Microscopic observation does not confirm this surmise. The constituents of the bast and wood tissues of Ranunculus are more various and more differentiated from one another than those of Pinus.

The bast is divisible into the protophloem and primary bast. The former lies next the outer sheath. It is composed of thin-walled, elongated, tubular cells, all similar to one another, and containing a watery sol of proteins enclosed by the thin film of protoplasm lining their walls. The protophloem is a very thin strand containing 
about a dozen or less of these narrow cells in its cross section. The constituents of the primary bast are of two kinds-wide prismatic tubes and narrow cells. The former have transverse endwalls. They are lined with a protoplasmic layer of great tenuity and contain a watery protein-sol and dissolved sugars. In the protoplasmic film may be detected small fragments of nuclear substance, and also minute starch grains. The end-walls of these tubes are perforated and act as sieve-plates. Through these perforations the contents of adjacent sieve-tubes are continuous. Often sieve-plates are formed on the longitudinal walls where two tubes are in contact with one another. The other constituents of

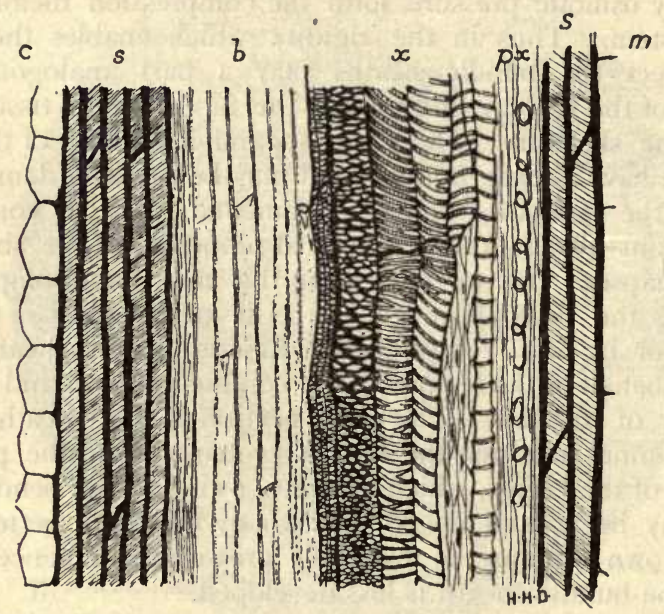

FIG. 76. - Ranunculus repens, conducting tract, longitudinal section, $\times 250$. $b$, bast; $c$, cortex; $m$, pith ; $p x$, protoxylem; $s$, sheath; $x$, wood.

the primary bast are the companion cells. They lie between the sieve-tubes, and are of the same length as they are: but in cross section they are much smaller. Their walls are equally thin, but their cavities are practically filled with protoplasm, leaving no visible vacuole. A large elongated nucleus completes their contrast with the sieve-tubes. A few flat tubular cells of equal length with the siave-tubes lie on the inner surface of the bast. They are procambial cells, which have failed to differentiate and have lost the power of dividing.

The wood or xylem, like the bast, is composed of two parallel filaments. That nearest the axis of the stem is the protoxylem. 
It is made up of very narrow capillary tubes. The walls of these tubes are lignified, and internal thickenings are to be seen on them. These thickenings in the protoxylem have the form of spiral bands or rings. The two forms often merge into one another, even in the same capillary tube. The length of these tubes is sometimes considerable, but they appear always to remain limited by the length of the original cell which produced them. Their protoplasm does not persist but their cavity is normally filled with water or dilute solutions. Consisting of the empty walls of single cells they are tracheids. Outside the protoxylem is the stouter filament, composed of the primary wood. The primary wood is built up of the following constituents: (I) Tracheids rather shorter than those in the protoxylem make up the greater part of this tissue. Their walls are moderately thick and lignified, and they are studded over with very minute bordered pits, smaller but essentially similar to those of the Pine. When the walls of the tracheids are very thick the tracheid is called a fibre. Vessels, very long tubes with lignified cell-walls which are closely covered with pits. To the formation of the vessels several, or many, cells contribute: as their side-walls become lignified and pitted their end-walls are absorbed, and so a continuous cavity is formed by the throwing together of a linear series of cells. Between and round about the tracheids, fibres and vessels are found: (3) Short prismatic cells with walls more or less lignified and retaining their cytoplasm and nucleus. These form the wood-parenchyma. There is often a layer of wood-parenchymacells round each of the larger vessels. This layer is frequently interrupted where the vessels come into lateral contact with one another or with tracheids or fibres. The location of the spiral and annular tracheids in the protoxylem is doubtless to be associated with the fact that they are developed from the procambium, while the stem is still elongating. 'Those forms of thickening manifestly easily accommodate themselves to this extension. The continuous thickening of the pitted walls occurs consequently only in that part of the wood which is formed subsequently to the period of great elongation; and we actually have a record of the change in the rate of elongation of the growing stem in the separation of the turns of the spiral thickenings. The innermost tracheids of the protoxylem have very steep spirals, while in the outer ones the spirals are flat and the turns close together.

In the veins of the leaf there are conducting tracts quite similar in structure to those of the stem. They are, however, more reduced in size not only by the diminution in the numbers of 
elements composing them, but also by the reduction in size of the elements themselves. The veins branch repeatedly in the leaf, tapering smaller and smaller, until they disappear altogether. The conducting tracts running in them suffer a similar diminution and finally also lose themselves in the green tissue. At their extreme tips the conducting tracts consist of one or two spiral tracheids accompanied by a similar number of narrow elongated cells representing the bast. Throughout the leaf the bast is on the under side of the wood, and this relative position is retained as the conduct:ng tract, now rendered more massive by the coming together of the various branches, passes into the rachis and leafstalk. Five or six of these tracts enter the stem from each leafstalk, and, after crossing the cortex, turn down the stem just outside the pith. The largest of the conducting tracts, coming down from the middle of the leaf-stalk, enter among the tracts already in the stem coming from higher leaves. They pass down a considerable distance in the stem before they merge with other conducting tracts. The lateral tracts of the leaves fuse immediately with the conducting tracts of the stem.

The green tissue of the leaf of the Buttercup is very distinctly differentiated into an upper stratum of palisade-parenchyma and a lower one of spongy-parenchyma. The cells of the latter are very irregular in form and are loosely put together, being in contact with their neighbours at only a few points. Between them are large intercellular spaces. As is usual, these spaces communicate with the outside through stomata in the lower epidermis. The cells of the palisade layer are comparatively regular in shape, being cylindrical with rounded ends. Between them are spaces which are continuous with those of the spongy-parenchyma. The walls of these two layers of cells composing the mesophyll are thin. The cells are green owing to the large number of chloroplasts in the layer of cytoplasm lining their walls. In this cytoplasm a nucleus is also usually seen. The cells are tense and rigid, owing to the osmotic pressure of the solutes in their vacuoles. The mesophyll is covered over above and below by the epidermis which resembles the epidermis of the stem. Stomata are for the most part found only in the lower epidermis. The veins containing the conducting tracts are composed of cylindrical cells lying parallel to the direction of the veins. The longitudinal walls of these cells are thick and evidently strong enough to resist a considerable tension. The finest ramification of the conducting tracts are embedded in the upper layers of the spongy-parenchyma.

The roots are developed inside the cortex of the stem or bulb, 
and from the conducting tracts of the stem branches are given off to supply them. As these branches pass out they undergo a twist and at the same time converge together. The protoxylem, which is innermost in the stem, passes to the outside of the wood in the root, while the filaments of primary wood drawing together occupy the axis of the root. In this way a core of primary wood forms the axis of the root, while three, four or five ridges of protoxylem run along its surface. In each groove, between the ridges of protoxylem, lies a strand of bast. A layer of cells - the pericycle - covers this cylinder of conducting tissue and over all is a thick coating of cortex. The innermost layer of the cortex limits the conducting

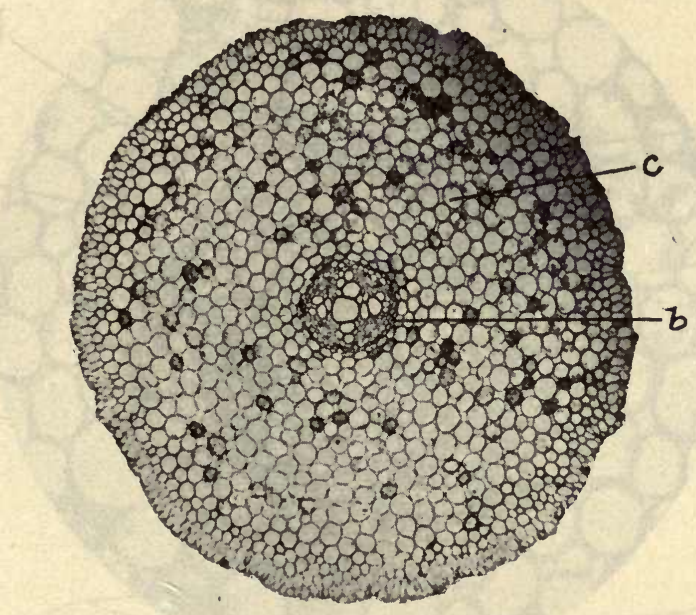

FIG. 77.-Ranunculus repens, root transverse section, $\times 50$. $b$, conducting tract ; $c$, cortex.

tract and is known as the endodermis. The outermost layer produces elongate tubular cells projecting from its surface-the roothairs. A root-cap is formed covering thegrowing tip of the root and facilitating the passage of the root among the soil particles by the exuviation of its outer dead cells.

From the description just given of the anatomy of the Buttercup it will be evident that the conducting tracts, or vascular bundles, are the channels of transport putting the absorbing surfaces of the root into communication with the photosynthetic leaves. The water entering at the root-hairs carries with it various nutritive substances in solution, derived from the soil, and is 
conveyed into the wood of the conducting strand of the root. There are special cells, passage cells, in the endodermis, opposite the protoxylem filaments, which appear to be exceptionally permeable and facilitate this transit. The traction of the evaporating surfaces in the leaves draws the water into the capillary tubes of the wood and upwards through the wood of root, stem and leaf.

To understand how this takes place it is necessary to emphasise a property of water which does not commonly come under

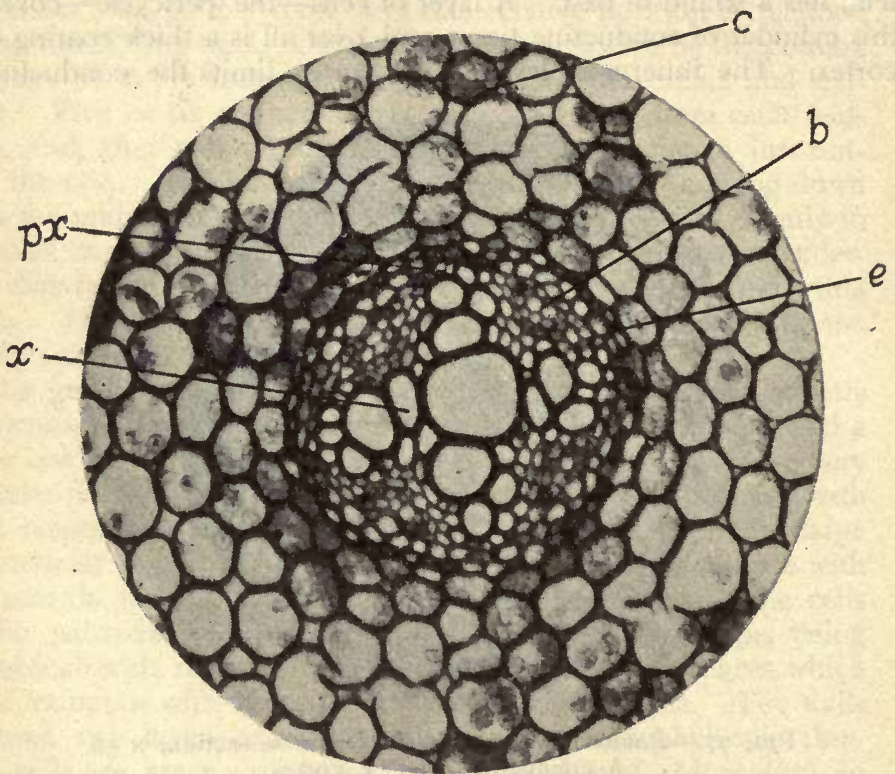

Fig. 78. - Ranunculus repens, conducting tract of root, transverse section, $\times$ I75. $b$, bast ; $c$, cortex ; $e$, endodermis ; $p x$, protoxylem; $x$, woodtracheæ.

notice. Water is capable of transmitting a pull and under suitable conditions may, by its tenacity, or tensile strength, be used like a rope or a wire to drag a weight. This tenacity usually escapes our observation because the necessary conditions to allow it to come into force are not realised.

I have here a glass tube about $80 \mathrm{~cm}$. long and a centimetre in diameter. It is bent into the form of a letter $\mathrm{J}$ and is furnished with a bulb blown upon the short arm. The capacity of the bulb is approximately the same as that of the long arm. The tube, 
which is closed at the end of the long arm, is partly filled with water. By inclining the tube the water may be made to occupy the long arm and the bend. Now if the tube be brought into an upright position the water will remain in the long arm. It is held there by the atmospheric pressure pressing on the surface in the short arm. This is easily proved by attaching an airpump to the short arm, and removing the atmospheric pressure.

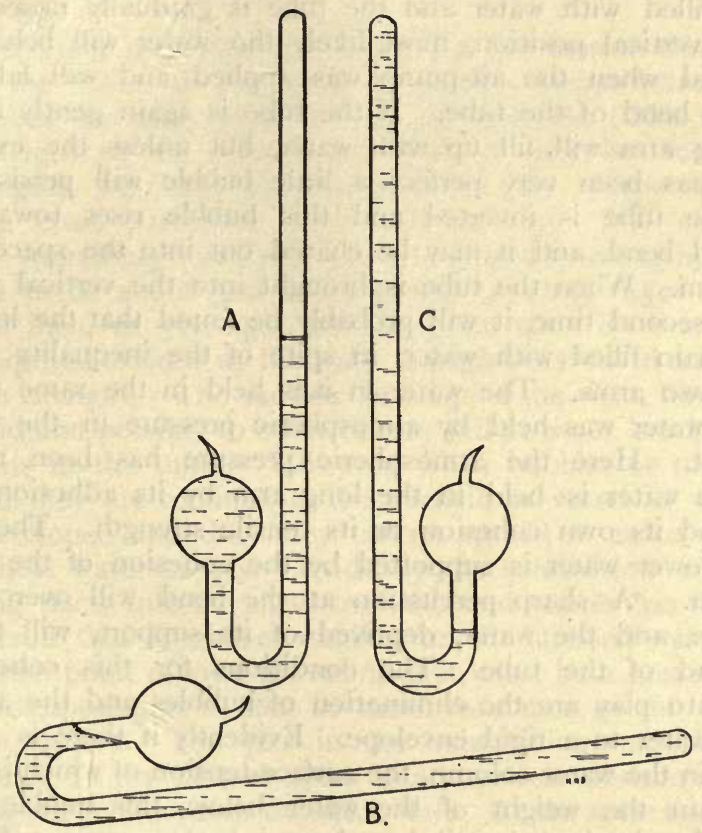

FIG. 79. - J-shaped glass tube; A, in vertical position, contained water lying in the bend; $\mathrm{B}$, inclined to bring the water into the long $\operatorname{limb} ; \mathrm{C}$, in vertical position, the water being retained in the long limb by its cohesion and by its adhesion to the glass.

Immediately the water falls down out of the long arm and occupies the bend rising to the same level in each arm. In this second similar tube special precautions have been taken to free the inner surface of the tube from grease, so that the water will wet it and adhere to it. Further, by prolonged boiling I have made certain that all dust particles in the water itself are also wetted. While the water in the tube was still boiling the 
tube was sealed off by melting the narrow steam-exit in a flame. This last operation secured that very little air remained in the tube. Hence the tube now acts as a water-hammer, i.e. when, by inclining it, the water is allowed to rush from end to end, its concussion makes a sharp metallic click. Evidently very little air is left in the tube to act as a cushion and to soften this concussion. If by inclining the tube the long arm is completely filled with water and the tube is gradually raised again into a vertical position, most likely the water will behave just as it did when the air-pump was applied and will fall down into the bend of the tube. If the tube is again gently inclined the long arm will fill up with water, but unless the expulsion of air has been very perfect a little bubble will persist in it. Now the tube is inverted and this bubble rises towards the upturned bend, and it may be chased out into the space in the short arm. When the tube is brought into the vertical position for the second time, it will probably be found that the long arm will remain filled with water, in spite of the inequality of level in the two arms. The water in it is held in the same position as the water was held by atmospheric pressure in the first experiment. Here the atmospheric pressure has been removed and the water is held in the long arm by its adhesion to the glass and its own cohesion or its tensile strength. The weight of the lower water is supported by the cohesion of the column above it. A sharp percussion at the bend will overcome its cohesion, and the water, deprived of its support, will fall into the bend of the tube. The conditions for this cohesion to come into play are the elimination of bubbles and the adhesion of the water to a rigid envelope. Evidently if there is a single bubble in the water column, the surface-tension of which is unable to sustain the weight of the water below, this bubble will be enlarged and enlarged until the column is broken across. Similarly if there is any spot where the water does not adhere to the wall, a bubble will be formed there and the column will be broken. It is of interest to notice that if we could enclose the bubble in a tiny cell, the outside of which would be wetted by the water, the complete breaking of the column would be prevented. It should further be observed that, although undissolved air in the form of a bubble, unless thus enclosed, breaks a tensile column of water, experiment has shown that dissolved air does not destroy its cohesion.

We will now turn our attention to the water in the plant. The moistened walls of the cells of the mesophyll lose water into the spaces of the leaf. To make good this loss water is 
drawn from the tracheids of the conducting tracts. The water in the tracheids wets and adheres to their walls. There are no bubbles in these tracheids, and consequently their watery contents are in a condition to exercise their cohesion and to transmit the pull, generated by evaporation, to the water in the neighbouring tubes, and similarly from these to the water lower down in the woody tubes of the leaf, stem and root. So the water is dragged up from the roots just as a rope or a wire might be.

In a small plant like the Buttercup the tension developed to raise the water in the plant is probably insignificant. But in tall trees the drag must be very considerable. Not only must the weight of the column of water (it may be 200-300 feet in length), but also the resistance of the narrow tubes and of the cross partitions must be overcome. The cohesion of water containing dissolved air, or its tensile strength, has been shown to be about 300 atmospheres, i.e. about $4500 \mathrm{lb}$. to the square inch, or $310 \mathrm{kgm}$. to the square $\mathrm{cm}$. So that even the pull required to draw up the water in high trees would not seriously tax its strength. The sudden bendings of the stem, however, due to wind and possibly to other causes, will at times break the water columns in the tubes. However, when bubbles or ruptures are formed they are confined to the tracheid or vessel in which they are developed and thus they cannot spread so as to cut across the whole water supply. Evidently the subdivision of the conducting tracts into the small tracheids and vessels has this important result that it confers stability on the stressed water. From any other point of view the subdivision must be a great disadvantage, interposing as it does so much resistance to the movement of the current. The pits in the subdividing walls lessen this resistance as the thinner spots interpose less obstruction to the moving water than does the thick wall. At the same time the thin membranes are not so strong to prevent the rupture spreading from the element which contains it. The structure of the bordered pit is a mechanism to give the necessary strength to the membrane and at the same time leave its permeability unimpaired. When there is water on both sides of the membrane and the membrane occupies a median position, the resistance is low; but if a rupture in the water develops on one side of it, the water is drawn away by the tension from that side, but adhering to the pitmembrane drags the torus against the dome. In this position the dome, which is thick and woody, supports the thin membrane and the torus fills up the perforation in the dome. Thus the 
surface of the water adhering to these structures receives all the support of the thick parts of the wall when a rupture occurs, while, as long as none develops, the resistance to the flow is that of the thin pit membrane.

The recognition that the water rising in the vessels and tracheids is usually in a state of tension shows why it is advantageous that the supports in these tubes should be of the form of internal hoops and spirals. These are forms of support preeminently suited to resist crushing forces such as the tensile adhering water would exert. It is interesting to note that while the strength of the tubular woody walls and their internal supports are utilised to counteract the tendency of the vessels and tracheids to collapse, it is the osmotic pressure exerted by the solutes which prevents the thin-walled mesophyll cells from crumpling in under the tension.

The evaporation, kept up from the leaf-cells by the inflow of heat there, is by far the most important factor in raising water in the plant; but at certain periods, viz. early spring, when the buds are opening, and before the leaf-cells can evaporate, and sometimes in warm nights when the moisture of the air checks evaporation, an upward current of water may be detected in the wood. This appears to be due either to the osmotic pressure of the solutes in the wood of the root drawing in water and raising it in the stem, or else to a pumping action located in the outer cells of the root. The action is spasmodic and temporary. The force exerted is called root-pressure. Probably its chief importance is to force water into those tubes where ruptures have occurred and so reestablish the columns of water which will transmit the tension to raise the transpiration current.

\section{PRACTICAL WORK.}

Make a sketch of a plant of Ranunculus bulbosus or of $R$. repens, showing the leaves, stem and roots.

Cut across the roots and immerse their cut surfaces in a watery solution of eosin. While transpiration is drawing up the solution into this plant, prepare a number of thin transverse sections of a root, a stem and a leaf. As they are cut transfer them into water in a staining glass so that they may be ready for examination.

In the transverse section of the root note the outermost layer forming the surface. It is the piliferous layer and its cells here and there grow out to form root-hairs. Within this is the thick cortex. In transverse section the cells appear rounded and are thin walled. Their cavities are chiefly occupied by starch grains. Between them small intercellular spaces are seen. The cortex is bounded internally by the endodermis. Its cells exhibit an oblong outline. Their walls are thickened and somewhat cutinised and so more or 
less impermeable. Passage cells, the walls of which are not cutinised, are seen at intervals in the endodermis. Within the endodermis is the conducting tract. Its outermost layer is the pericycle, composed of small thin-walled cells retaining their protoplasmic contents. The vessels and tracheids of the root occupy the centre of the cross section and appear in it as a stellate patch. The primary wood forms the central part of this patch while the spiral tracheids of the protoxylem form the tips of the rays of the star. There are usually four such rays of protoxylem. They are radially opposite the passage cells of the endodermis. The bast appears as narrow thin-walled elements between these rays.

The transverse section of the stem appears as a perforated disc, the greater part of which is composed of fundamental tissue. It is bounded on the outside by the epidermis. The majority of its cells are almost square in transverse section. Their outer walls are thickened and cutinised. Occasional tapering hairs are seen which are out-growths of single epidermal cells. Unlike the root hairs their walls are thick. Within the epidermis are the cortex, medullary rays and pith. The characteristics of the cells composing these tissues have already been described. The structure of the vascular bundles and their sheath is easily made out. Careful drawings should be made of the whole cross section under the low power showing the position of the bundles and of parts of the various tissues under the high power.

Drawings should also be made of the transverse section of the leaf, showing the characteristics of the epidermis, palisade and spongy parenchyma.

In addition to these sections, sections should be made of the stem of the plant whose roots have been immersed in the eosin solution. They should be cut with a $d r y$ razor and transferred to a $d r y$ slide. Without covering them they should be examined with a low power. It will be seen that the eosin, which has entered the roots, has been drawn up in the wood and occupies the cavities of the tubes of the wood. It stains their walls, and in time spreads to the adjacent tissues. The observation shows that the tubes are the water carriers in the tract. 


\section{LECTURE XXVI.}

LiKe the Pine the Buttercup reproduces itself by means of seeds. The seeds of the Buttercup are formed in connection with the flowers. The flowers are produced at the ends of the branches of the upright stem. The ends of these branches terminate in a coneshaped enlargement. This is the axis or thalamus of the flower on which the specialised floral leaves are inserted. The leaves belong to four different categories.

The five or six outermost or lowest are ovate and pointed; they

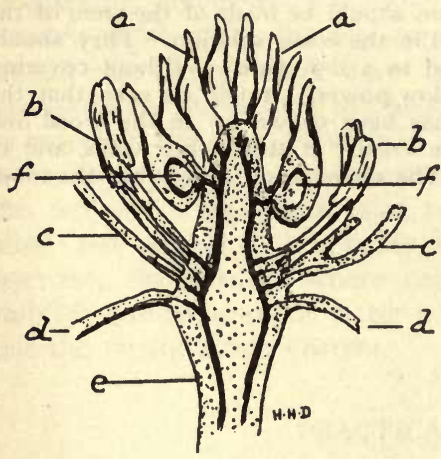

FIG. 80,-Ranunculus repens, flower,

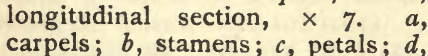
sepals; $e$, axis showing conducting tracts ; $f$, ovules. are attached by a broad base to the lower part of the cone-shaped axis, where its diameter is greatest. They are pale green. Each is called a sepal and they form together a circle, or whorl, round the axis, known as the calyx. In Ranunculus bulbosus their outer ends are bent backwards along the stalk of the flower. In $R$. repens each is boat-shaped and is directed obliquely upwards. While the young flowerbud is developing the sepals cover it over, and only when it is approaching maturity do they bend backwards and open to allow the inner leaves to expand. Hence it is believed that their function is to protect the soft tissues and immature organs of the developing flower.

Within and above the calyx is the corolla. It is composed of the same number of petals as the calyx has sepals. The petals are attached to the axis just above the sepals and they alternate with the sepals. They are brilliant yellow, glossy on the upper surface and dull beneath. The yellow colour is due to a pigment 
held in special protoplasmic bodies, the chromatophores, in the cells. They are narrow at their base, by which they are attached to the axis, and their distil end is expanded. There is usually a curved indentation on their rounded outer end. Just at the base on the upper surface there is a minute pocket formed by a scale attached to the petal, with its opening directed upwards. The inside of the pocket is moistened with a sugary, viscid juice. Veins may be seen ramifying through the petals just as they are visible in the foliage leaves.

Above the whorl of petals a large number of filiform organs are attached to the sloping side of the conical axis. These are the stamens. Their points of attachment are arranged in spiral lines upon the axis. Each stamen is formed of a tapering white stalk, the filament, bearing at its top an ovoid enlargement, the anther, yellow in colour and with a deep longitudinal groove. On each side of this groove is a bulging mass, the anther-lobe. When the stamen is mature each anther-lobe splits or dehisces longitudinally and emits a yellow powder, the pollen.

The origin of a stamen is similar to that of a foliage-leaf. It begins as a small rounded excrescence on the side of the axis formed of undifferentiated cells. Gradually the distal part of the excrescence enlarges and the beginnings of the filament and anther may be discerned. In the anther a shallow, longitudinal groove appears dividing it into two lobes. The growth of the tissue forming the bottom of the groove widens it and separates the two anther-lobes. This tissue is called the connective. In the connective and filament a small conducting tract is developed which makes connection with the conducting tracts of the axis. In each anther-lobe two columns of cells differentiate. The cells forming them are distinguished from their neighbours by their larger nuclei, more granular contents and larger size. Round each column is a layer of crushed cells called the tapetum. The columns are called the archesporia and give rise to the spores or pollengrains. The component cells are the pollen-mother-cells, which are similar in their characteristics to the pollen-mother-cells of the Pine and the spore-mother-cells of the Archegoniate. As the archesporia increase in size they come to occupy more and more of the anther-lobes so that the sterile tissue of each anther-lobe separating its two archesporia becomes a narrow partition. The nuclei of the pollen-mother-cells divide twice and so give rise to four nuclei, tetrahedrally arranged in each cell. The cytoplasm segments to form round each of these nuclei a globular mass. This develops a cell-wall, the outer surface of which is roughened 
with minute tubercles of cutinised cellulose. Each of the cells so formed is a pollen-grain. While the pollen-grains are being developed the filament of the young stamen elongates, and changes occur in the outer layers of the anther-lobes. The cells forming the tapetum are absorbed, apparently being used up in the formation of the pollen-grains and their walls. The pollen-grains thus come to lie loose in the four cavities - the four pollen-sacs-two in each lobe of the anther. The superficial layers of the anther form the outer walls of these pollen-sacs, and are composed of the epidermis and the fibrous layer. The walls of the cells of this layer are thin but strengthened by the deposit of fine ridges of lignified cellulose on their inside. These ridges are absent from the outer wall. They form reticulations on the inner wall, and

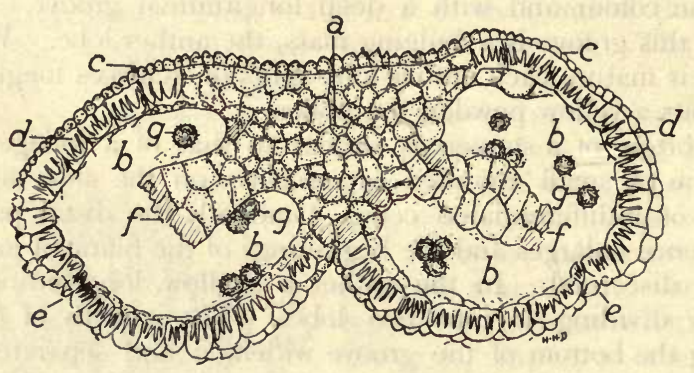

Fig. 8r.-Ranunculus repens, anther, transverse section, $\times$ 70. $a$, connective ; $b$, pollen-sacs; $c$, epidermis; $d$, fibrous layer; $e$, line of dehiscence; $f$, broken partition between pollen-sacs; $g$, pollen-grains.

they project out along the radial walls in a radial direction. It will be understood that with this form of support the outer walls of the fibrous layer are free, with the loss of water in ripening, to crumple, while the inner walls remain rigid. This leads to a strain, and to a tendency to a change in curvature of the wall of the pollen-sac which is relieved finally by the tearing of the wall. The place where the rupture will occur is decided by the weak coherence of the cells of the epidermis along a band just opposite the partition which separates the two pollen-sacs of the anther. The split in the wall along this line and the recurving of its parts simultaneously break into both of its sacs and set free the pollen.

Above the tapering, cone on which the stamens are set the axis expands into a small rounded knob. On this are set a number of carpels. The carpels have the form of flasks flattened below on 
two opposite sides. The necks are tapering and obliquely bent. Viewed from one of the flattened sides, the body is unsymmetrical and shows a bulge on one side. This side (abaxial) is turned away from the axis while the neck or style is first bent towards the axis and then turns outwards. The style terminates in a rough surface formed of rounded cells which secrete a sticky fluid. This surface is called the stigma. The swollen body of the flask-shaped

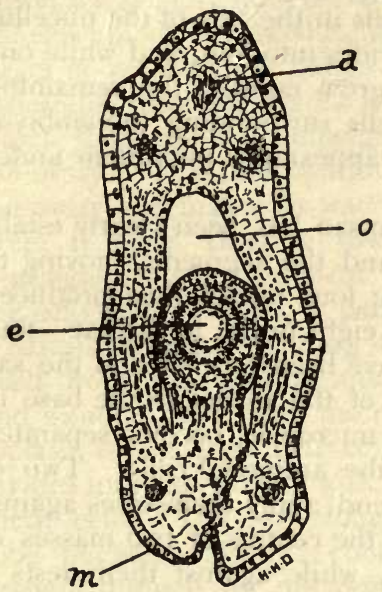

Fig. 82.-Ranunculus repens, young carpel before fertilisation, transverse section, $\times 45$. $a$, conducting tract in mid-rib; $e$, embryo-sac in ovule attached by stalk to the margin $(m)$ of the carpel; 0 , loculus or cavity of ovary.

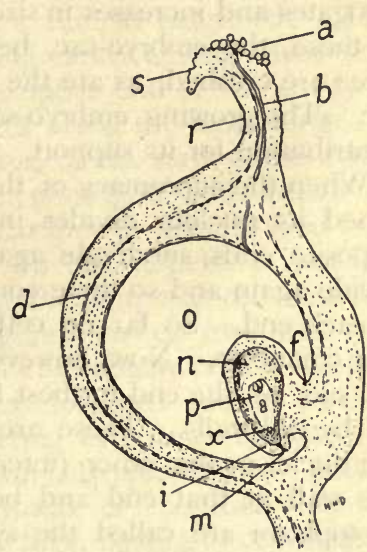

FIG. 83.- Ranunculus repens, carpel, longitudinal section, $\times I_{3}$. $a$, pollen-grains germinating on stigma $(s) ; b$, pollen-tubes passing down style $(r) ; d$, conducting tract in mid-rib of carpel; $f$, stalk ; $i$, integument ; $m$ micropyle; $n$, nucellus of ovule; 0 , cavity, or loculus, of ovary; $p$, embryosac ; $r$, style; $s$, stigma ; $x$, ovum.

carpel is called the ovary. A cross section of the ovary shows that it encloses a cavity. The wall of this cavity is a few layers of cells thick and along that side of the ovary, which is turned away from the axis, is seen a ridge or vein in which a conducting tract runs. There is a groove in the inner (adaxial) side of the ovary and on each side of the groove a smaller conducting tract is found. Enclosed within the cavity of the ovary and attached to its adaxial side is an egg-shaped body, the ovule. It is attached by a stalk 
which is bent back on itself where it enters the ovule. The ovule consists of a central egg-shaped mass of cells-the nucellusenveloped in two coverings, the integuments. The integuments do not close over the top of the nucellus but leave a passage leading to it. This passage is called the micropyle. Owing to the bend in the stalk the micropyle is directed towards the base of the stalk. A conducting tract runs from the base of the nucellus down the stalk and joins one of the two conducting tracts in the adaxial side of the ovary wall. One of the cells in the axis of the nucellus elongates and increases in size. It divides into four, and while one of these, the embryo-sac, begins to grow rapidly, the remaining three are crushed, as are the other cells surrounding the embryosac. The growing embryo-sac to all appearance puts them under contribution for its support.

When the supremacy of the embryo-sac has been clearly established its nucleus divides into two and the segments, moving to opposite ends, subdivide again. The four nuclei thus produced divide again and so there come to be eight nuclei in the sac-four at each end. So far no cell-walls have been formed and the sac is a cœnocyte. Now, however, three of the nuclei in the base of the sac, i.e. the end furthest from the micropyle, become separated off by cell-walls. These are called the antipodal cells. Two of the nuclei in the other (micropylar) end apply themselves against the wall at that end and becoming the centres of two masses of protoplasm are called the synergidæ, while against them rests a third nucleated mass of protoplasm - the ovum. The remaining two nuclei (polar nuclei), one from each end of the sac, move to the middle and coming into contact with one another ultimately fuse together and form the fusion-nucleus.

The lower outermost stamens are the first to ripen and dehisce, thus exposing their pollen on the open anther-lobes. This occurs before the styles and stigmas of the carpels of the flower have emerged above the closed anthers of the upper stamens.

Ripening proceeds from below upwards and when the uppermost anthers are opening the stigmas of the carpels come to maturity and are covered with the sticky fluid.

Various small insects, flies, beetles, and even bees visit the flowers of the buttercup to gather the honey produced in the pockets at the base of the petals. In visiting the freshly opened flowers they rub against the ripe anthers and become dusted with pollen which they carry away with them as they leave the flower. When they alight on a more mature flower, the under sides of their bodies rub off some of the pollen thus acquired on to the stigmas 
of the carpels, where it adheres. These adhering pollen-grains 'may usually be seen if mature stigmas are microscopically examined.

Just before the dehiscence of the pollen-sacs the single nucleus of the spherical pollen grain divides into two and a protoplasmic partition develops dividing the grain unequally into a small lenticular cell containing one of these nuclei and leaving over the greater part of the grain to be tenanted by the other. In this condition the pollen-grain is transferred to a stigma by an insect. It adheres among the papillæ, absorbs nutriment and immediately the larger of its two cells begins to form a tubular outgrowth. This penetrates into the stigma and, passing down among those cells which form the style, emerges in the upper end of the cavity of the ovary. The tube continues its path along the inner surface of the ovary and makes its way to the base of the stalk of the ovule. Here it turns abruptly towards the micropyle and enters it. Throughout its development this tubular growth absorbs food from the cells among which it penetrates. In fact it behaves just like a parasitic fungus. During its passage the lenticular cell detaches itself from the wall of the pollen-grain, passes into the tube and wanders down after the nucleus of the tubular cell. The protoplasm of the lenticular cell becomes elongated and its nucleus divides into two, which with the covering cytoplasm become two sperm-cells. The two elongated sperm-cells pass by the pollentube nucleus. By the continued growth of the tube they are brought through the micropyle. The tube, having forced through what remains of the nucellus applies itself to the embryo-sac. At the point of contact the wall of the sac and tube dissolve and the two sperms passing the synergidæ enter the sac. One fuses with the ovum and the other with the fusion-nucleus.

The oosperm formed of the united sperm and ovum gets a cellwall and proceeds by nuclear and cell-division to form a short column or row of cells, fixed in the upper end of the embryo-sac by one end and projecting into the middle of the sac where it terminates in a globular cell. The latter is called the embryonic cell and the rest forms the suspensor. The embryonic cell subdivides and becomes a bilobed mass. The two lobes become the two seed-leaves (cotyledons). In the groove between them the apex of the stem of the embryo develops. The root develops at the opposite pole where the embryonic mass abuts on the suspensor. The conducting tract of the root develops from the former and its cortex and root-cap from the latter.

As the embryo differentiates the ovule becomes the seed. The fusion-nucleus in the embryo-sac divides and its products continue 
to divide, while the cytoplasm of the embryo-sac increases in bulk at the expense of the nucellus and of material transported to it by the conducting tract in the stalk. Cell-walls are produced which subdivide the embryo-sac into nucleated cells. Thus the sac becomes filled with tissue, the endosperm. Meanwhile the nucellus is reduced to a membranous sheath while the outer integument is hardened and becomes the seed-coat.

Fertilisation also induces changes in the carpel which hardens and becomes a membranous coating-the fruit-adhering to the outside of the seed.

After ripening the seed passes into a dormant state, in which the suspension of vital activities seems to be complete. Thus in many dormant seeds no respiration can be detected. The protoplasm also is in a particularly stable state. In this connection it may be mentioned that some seeds, when thoroughly dry, may be-exposed to a temperature of over $120^{\circ} \mathrm{C}$. without injuring their power of germination. The addition of moisture to seeds in this state of suspended animation restores to the embryo its normal sensitiveness and enables it by respiration to set free energy stored in its own cells and in the surrounding endosperm. The water seems to act as a catalyst to the various reactions which constitute embryonic metabolism. By the absorption of the endosperm material is found for growth, and the root elongates and forces its way through the seed-coat. In response to the stimulus of gravity the emerging root turns downward into the soil. The seed-leaves are then drawn out of the empty seed and being provided-with chloroplasts make the young plant independent.

Consideration of the structure and life-history of the Buttercup shows that it is more nearly comparable with the Pine than with the Archegoniates. The absence of cambial or secondary thickening makes the structure of stems and roots appear more simple, but the actual composition of the conducting tissues, wood and bast is more complex - a greater variety of elements contribute to form them. It is, however, in the reproductive organs that we find the most marked differences. The events of the life-history show that the pollen-grains and embryo-sacs of the Buttercup, like those of the Pine, are homologous to the spores of the Archegoniates.

Thus the stamens and carpels are respectively microsporophylls and megasporophylls, and the pollen-sacs and ovules may be homologised with microsporangia and megasporangia. The microsporophylls (stamens) of the Buttercup are, however, more divergent from the sporophylls of the Ferns than are those of the 
Pine. In the megasporophylls the divergence is still greater. The closed ovary is practically a new organ furnishing special protection to the megasporangium, but involving other changes, viz. the formation of the receptive stigma, the nutritive and guiding style and the prolonged growth of the pollen-tube (male gametophyte). The megasporophyll is still evidently in its origin a leaf, but the approximation of its margins over its upper surface and their fusion changes the flat leaf into a closed capsule. This change may be followed in the development of each megasporophyll (carpel), and it is only in the final stages of development that the closing of the ovary is completed, and the condition, characteristic of the highest group of seed plants, viz. the inclusion of the ovules and seeds in closed carpels, is attained. Hence it is that this group is called the Angiosperms in contradistinction to those which, like the Pine, have the ovules and seeds exposed and are styled the Gymnosperms.

The development of special attractions for insects in connection with the spore producing axis or flower is a modification of farreaching importance, and being combined with a suitable disposition of parts evidently secures the direct transference of the microspores (pollen) to a position which will ensure fertilisation. This avoids the wasteful production of microspores, which is necessary when the arrival of the microspores into proximity with the megaspores is fortuitous, as in the case of the Pine. Thus it has been found that the bright colours and wonderfully diverse shapes and mechanisms of the flowers of the Angiosperms have direct relation with this insect-pollination and are in the vast majority of cases only found in those which are dependent on insects for the transference of their pollen to the stigma. On the other hand, where the wind is the agent for pollination inconspicuous flowers and different mechanisms prevail such as are found generally in the Gymnosperms and also in certain of the Angiosperms.

The increased complexity of the sporophyte of the Pine is associated with a reduction and simplification of the gametophyte. In the Buttercup the reduction of the gametophytes has gone a step further. The microgametophyte or male gametophyte at its maximum development is a minute cœnocytic hypha, containing three nuclei, two of which are the nuclei of the sperm-cells. No vegetative tissues are developed and no cellular sexual organs. Such a complete absence of differentiation is rendered possible by its holozoic nutrition and its parasitic habit. It is remarkable that in spite of its dimensions being greater than those of the micro- 
gametophyte of the Pine its differentiation is further reduced. The reduction has also affected the megagametophyte. Instead of the ovoid cellular mass found in the Pine, it retains the conocytic condition characteristic of the early stages of the female gametophyte of the Pine, and even when the ovum is mature there is no cellular archegonium formed for its reception. It lies in the parasitic cœnocyte just like the ova of some of the parasitic Fungi. The reduction in the number of the ova is also to be noticed, and the fusion of the second sperm-nucleus with the fusion-nucleus and that of the two polar nuclei may indicate that in these nuclei are to be recognised other ova. If this interpretation is correct the endosperm or food material resulting from this fusion would be homologous to a second embryo. Hence, it would not be homologous to the endosperm of the Pine. In fact, the former would be homologous to a sporophyte, while the latter is homologous to a gametophyte.

\section{PRACTICAL WORK.}

Make careful sketches of the flower of the Buttercup, viewed from above and below, showing the numbers and the relative positions of the floral leaves.

Slice the flower in two parts, beginning with the flower-stalk and passing upwards through the axis. Sketch the surface so made, showing the axis and flower-stalk in longitudinal section and the exact attachment of the various parts.

Isolate sepals, petals, stamens and carpels, and using a simple lens sketch each from various aspects. Name the parts of the stamens and carpels. Cut cross sections of the stamens and carpels in an opening bud. Mount in water and cover. Sections passing through the anthers will show the four pollen-sacs containing the rounded pollen-grains, the tapetal layer, the wall of the sacs, the connective and its conducting tract. A drawing should be made showing these parts. Sections passing through the ovary will show its wall with mid-rib, its fused margins and in favourable specimens an ovule attached to the inner surface of one of these margins. The stalk, nucellus and integuments of the ovule should be made out and sketched. 


\section{LECTURE XXVII.}

THE first leaves formed on the young sporophyte are usually called its cotyledons. In the Gymnosperms a variable number of these (always exceeding one) arise in a circle surrounding the apex of the stem. In the greater part of the Angiosperms there are just two seed-leaves which arise as lobes on the embryo at opposite sides of

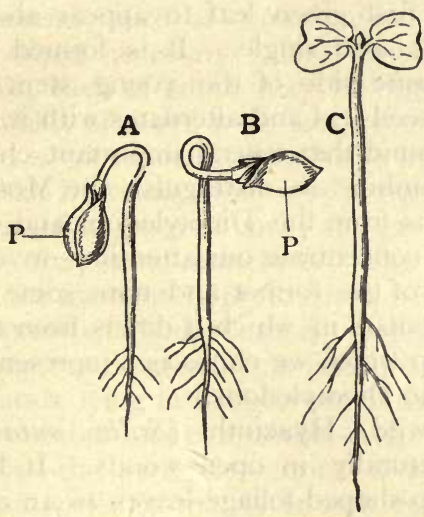

Fig. 84.-Seedling Dicotyledon. Two seed leaves (cotyledons) at $c$. (From Farmer's Practical Introduction to the Study of Botany.)

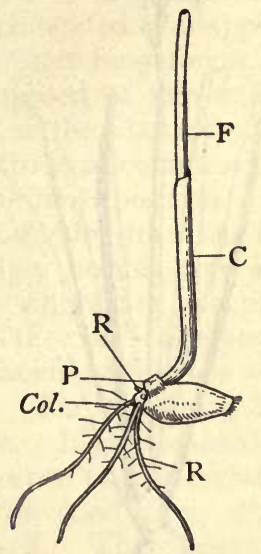

FIG. 85.-Seedling Monocotyledon. $c$, first leaf to emerge from the seed leaving the single cotyledon still enclosed. (From Farmer's Practical Intro. duction to the Study of Botany.)

the groove, from which the apex of the young embryo's stem differentiates. In their position they resemble those of the Gymnosperms but to all intents and purposes their number is fixed at two. Besides these, there is an important section of the Angiosperms in which the embryo develops first as a club-shaped mass at the lower end of the suspensor and the distal half of this mass differentiates 
as the first leaf or cotyledon. At the base of it on one side of the embryo is a group of small cells which differentiates as the apex of the stem. In this embryo the apex of the stem is lateral and the single cotyledon terminal. Angiosperms which develop in this way are called Monocotyledons while those which have an embryo with two seed-leaves and a terminal apex are called Dicotyledons. The difference is most marked at germination and is then readily recognisable without microscopic dissection. Later on, the two

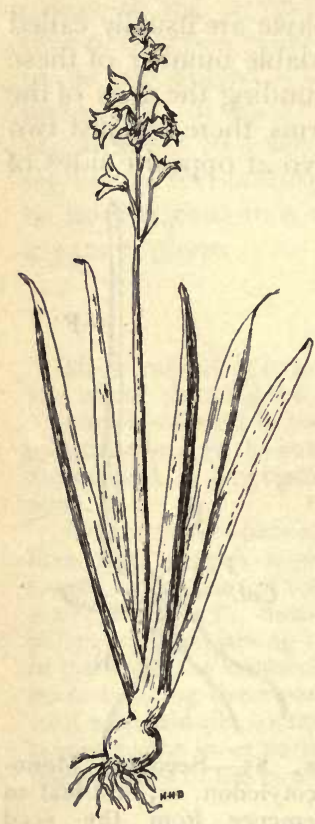

FIG. 86.-Scilla nutans. seed-leaves of the Dicotyledons are withdrawn from the seed and appear above ground usually as two entire green leaves, or remain within the seed and are easily recognised when the seed-coat is removed. But the single seed-leaf of a Monocotyledon remains attached as a rule in the seed and the first green leaf to appear above ground is also single. It is formed on the opposite side of the young stem to the first seed-leaf and alternates with it.

It is found that several important characters combine to distinguish the Monocotyledons from the Dicotyledons and we will now concentrate our attention on our example of the former and note some of the particulars in which it differs from the Buttercup which we chose as a representative of the Dicotyledons.

The wild Hyacinth (Scilla nutans) grows naturally in open woods. It has long strap-shaped foliage-leaves $20-40 \mathrm{~cm}$. long and $\mathrm{I}^{\cdot} 5^{-2} \cdot 0 \mathrm{~cm}$. wide. From among these rise slender flowering stems each supporting near its top eight or ten bellshaped blue flowers. The flower-stem bends gracefully under their weight. Each of these flowers is attached to the stem by a short flexible stalk which springs from between two small blue-purple scales (bracts).

If a plant is dug up it will be found that below the surface of the soil the leaves become white and are expanded round a bulbshaped mass. At the base of this bulb are found membranous scales which are the remains of the bases of the preceding year's foliage-leaves. From the base of the bulb issue a number of cordlike whitish-brown roots. 
If the bulb be split down longitudinally it will be seen that the leaves and outer scales are attached to a low cone- or cushionshaped mass of tissue tougher than that composing the leaves. This is the stem and from its broad base the roots spring. The flowering stern is a prolongation of this cone. In the angle of the uppermost foliage-leaf of the cone is a bud which will develop into the flowering stem of next year. Its base will form a bulb which will be coated over with the remains of the leaves of the current season.

The roots have a structure similar to those of the Buttercup. The conducting tract occupies the axis and is enveloped in a thick cortex. This distribution of tissues evidently has relation to the external conditions to which the root is subjected. It has to follow a tortuous course among the soil particles; hence the axial location of the conducting tract built up of elongated overlapping elements, such as would resist longitudinal stretching forces, is rendered necessary. The cortex, being composed of thin-walled cells, each of which may be distorted owing to the elasticity and ductility of its cell-wall, can accommodate itself to local compressions and is consequently suitable to occupy the outermost position. As in the Buttercup, the woody strand of the conducting tract has the form of a grooved core ; the grooves and ridges on this core are, however, more numerous. The crests of the ridges are occupied by the filaments of protoxylem, while the rest of the core is composed of primary wood, consisting principally of tracheids and vessels with some wood-parenchyma interspersed between. The bast forms fine strands lying in the grooves of the woody core. It is composed of sieve-tubes and companion-cells. A single layer of undifferentiated cells-the pericycle-forms a sheath over the conducting tract. From cells of this layer new roots are formed. They originate opposite one of the protoxylem-filaments and shove their way outwards through the surrounding cortex. When they emerge at the surface the ruptured cortex forms a collar at their base. The cortex resembles a thick sleeve covering the conducting tract. It is formed for the most part of rounded thin-walled cells, including between them angular intercellular spaces. It is bounded on the outside by the piliferous layer, which is a superficial layer of cells, some of which grow out at right angles to the surface and form absorbent hairs. They are very thin-walled. Their walls are lined with a very delicate film of protoplasm which in turn encloses a large watery vacuole occupying the larger part of the cavity of the cell. Their thin walls mould themselves to the surrounding soil particles and often adhere closely to them. On the inside the 
cortex is limited by the endodermis. This is a single layer of cells with cutinised cell-walls which fit together closely and seem to form a physiological barrier between the cortex and the conducting tract. The air passages in the cortex are cut off by the endodermis from coming into contact with the elements of the wood and bast. Here and there a cell of the endodermis is not thickened or cutinised like its fellows. It seems to have the function of transmitting water from the cortex to the wood and is in consequence of this

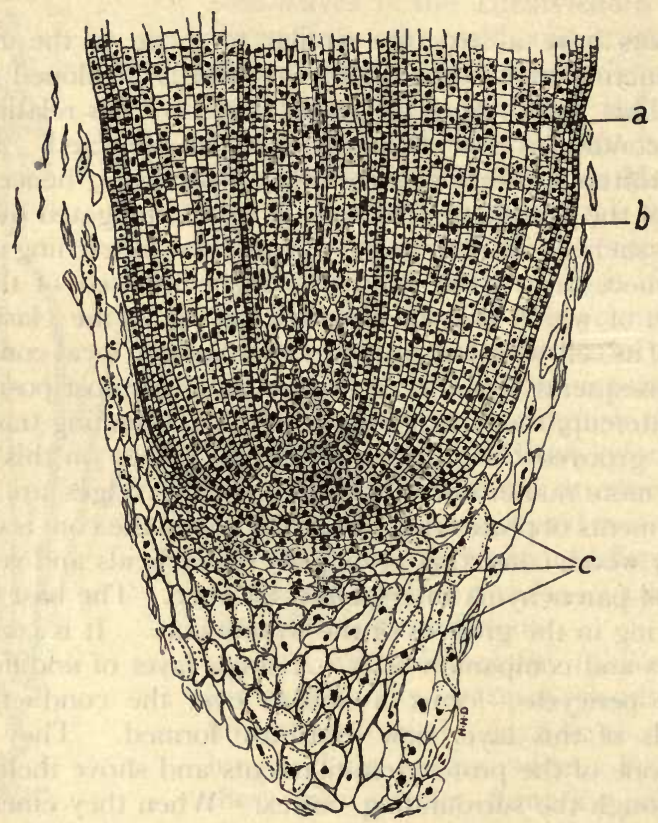

FiG. 87.-Tradescantia virginiana (a typical monocotyledon), root-apex, longitudinal section, $\times 80$. $a$, procambial tract; $b$, cortex; $c$, root-cap.

called a passage-cell. The passage-cells occur radially opposite the protoxylem-filaments.

The root grows at its tip, which is formed of a rounded cone of undifferentiated cells. The apex of this cone forms, and is covered over by, a cap of cells in various stages of cisintegration. The inner layers of this root-cap next the growing tip are rounded cells in active life; those further out loosen from one another, and the outermost part connection with one another easily and are cast off. 
Behind the root-cap the growing tip is made of cells which during the active season are constantly dividing, and the cells so produced add to the inner layers of the root-cap in front and to the cortex and conducting strand behind. Soon after formation the vacuoles of those cells, which have been added on to the cortex and conducting tract, increase greatly in volume and the cells are stretched to many times their original size. This growth adds rapidly to the length of the root and pushes the apex on through the soil, the exuviated outer layers of the root-cap lubricating its way.

The low conical stem of the bulb is composed largely of fundamental tissue formed of rounded cells filled with starch. Through this pass the conducting tracts or vascular bundles coming from the leaves. Their paths are irregular and they make frequent connections with one another. In the lower parts of the stem branches are given off from the conducting tracts to the roots. The arrangement of tissues in the flowering stem is more orderly. The mass of the stem is composed of fundamental tissue. Its epidermis of elongated cells is covered with a cuticle. The cuticle is perforated by numerous stomata and the stomata are each bordered by two guard-cells. The arrangement of the guard-cells towards one another is very like that found in the Buttercup, but the slits of the stomata are all parallel to the axis of the stem. Below the epidermis is a layer of tissue formed of prismatic elongated cells. The walls of these cells are specially thickened at the angles so that in cross section the cavity of the cell appears circular while the outline of the cell-wall is polygonal. Such tissue is called collenchyma. It is usually found on the outside of erect cylindrical organs, where the tissues are exposed to great stretching forces. The cells of the collenchyma contain chloroplasts as do the rounded thin-walled cells of the inner cortex inside them. The central fundamental tissue is made up of short cylindrical cells with rounded ends and they possess few chloroplasts but often contain starch grains and other store materials.

The conducting tracts from the bracts and flowers pass obliquely, downwards and inwards towards the axis as they enter the stem. Before reaching the axis of this organ they turn sharply downwards and then gradually turning outwards run down the stem. When in their course they reach the inside of the cortex they run vertically downwards and fuse with their fellows coming from other bracts. During their downward path they pass round the stem so that they follow a spiral course. This path of the conducting tracts is very different from that which is found either 
in the Dicotyledons or in the Gymnosperms. 'The difference is most easily appreciated in a transverse section. As we have seen, the conducting strands of a Dicotyledon form a circle in a transverse section of the stem. In a Monocotyledon, on the other hand, they appear irregularly scattered. Some are to be seen in the central region of the section. They have entered from leaves attached just above the level of the section. Those near the cortex are

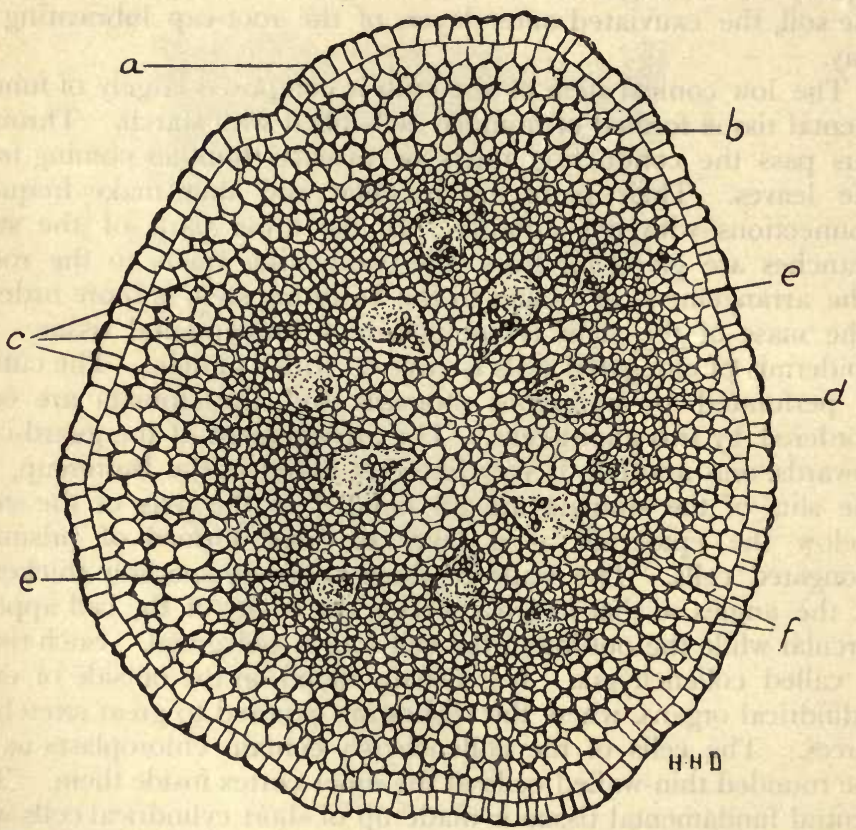

FIG. 88. - Scilla nutans, flower-stalk, transverse section, $\times$ 45. a, cuticle; $b$, epidermis; $c$, fundamental tissue; $d$, conducting tract or vascular bundle; $e$, wood or xylem; $f$, bast or phloem.

those which have come from leaves inserted at a higher level. The conducting tracts are largest just after they enter the stem and they taper gradually downwards. Hence the conducting tracts in the central region of transverse section are larger than those nearer the periphery. In structure the conducting tracts of the Monocotyledons resemble generally those of the Dicotyledons, but they never possess a cambium layer between the wood and the bast 
and usually, the number of elements being more limited, there is greater regularity in their arrangement. As a tract or bundle is traced downward it is found that the wood and bast are further reduced while the sheath increases in thickness. The conducting tracts of the flowering stem pass down and unite with those of the stem of the bulb so that the whole conducting system is continuous throughout the plant.

The leaves of the wild Hyacinth are constructed very much in the same way as those of the Buttercup. They consist of a middle layer (mesophyll) of green cells put together loosely and covered over, above and below, by a colourless epidermis. The epidermis also is coated with a cuticle, but it has no hairs and presents, consequently, a smooth and burnished appearance. The mesophyll is somewhat more porous towards the under surface, but the differentiation into palisade and spongy parenchyma is scarcely apparent. In the disposition of the conducting tracts in the leaves of the two plants there is a notable difference. In the Buttercup the conducting tracts branch in the mesophyll and form very fine branches which anastomose, or join together, irregularly and form a network in the leaf. In the Hyacinth the chief conducting strands run parallel to the mid-rib and here and there send off oblique branches uniting with a neighbour. This difference is characteristic of the two groups to which the plants belong. As the large conducting tracts lie in the veins of the leaves, we find that parallel venation is characteristic of the leaves of the Monocotyledons while reticulate (netted) venation is characteristic of the leaves of Dicotyledons. The arrangement of the stomata on the leaves constitutes also a method by which the epidermis of a Dicotyledon may be generally distinguished from a Monocotyledon. In the former the slits of the stomata are oriented fortuitously, in the latter they are parallel to the mid-rib. There are stomata on both the upper and lower epidermis of the Hyacinth.

The structure of the flowers must next engage our attention.

The flower-stalk is continued upward as the axis, to which the floral leaves are attached. In the Hyacinth the outermost floral leaves form a whorl of six. They are blue in colour ${ }^{1}$ and each is ovate in shape. The distal tapering end of each is recurved so that the whorl forms a structure like a vase surrounding the inner parts of the flower, and performs not only the protective functions of the calyx but also the attractive functions of the corolla. Such

${ }^{1}$ The pigment in this case is not held in chromatophores, but is dissolved in the vacuoles of the cells. 
an outer whorl which is not distinguishable into calyx and corolla is called a perianth. Sometimes the segments of the perianth of the Hyacinth are slightly coherent together by their bases. Immediately above the perianth are six stamens. They are attached to the axis, but sometimes adhere to the base of the segments of the perianth. Their filaments are white and tapering while their anthers are blue. The tapering end of the filament is joined to the middle of the connective so that the anther seems to pivot at its middle on the top of the filament. The anthers dehisce longitudinally and shed the pollen.

The segments of the perianth and the stamens have a similar origin to the sepals, petals and stamens of the Buttercup, i.e. their early stages resemble the early stages of foliage-leaves. Differentiation as perianth segments and stamens occurs later. After the anthers and filaments of the stamens have been outlined the four columns of sporogenous cells develop in the anthers, the pollensacs, each containing one column, are formed and the connective is differentiated. The wall of the pollen-sac, as in the Buttercup, is divisible into two layers, the epidermis and fibrous layer. The latter secures the dehiscence of the pollen-sac by a mechanism similar to that which has been described in the pollensac of the Buttercup. The pollen-grains also arise in the mothercells or sporogenous cells in tetrads just like those of the Buttercup.

Above the stamens and on top of the axis is a globular capsule tapering upwards into a delicate column ending bluntly in a rough and sticky surface. The capsule is the ovary: the column, the style: and the rough and sticky ending, the stigma. When the ovary is cut across it is seen to be divided into three compartments (loculi) by vertical partitions. 'There is a groove on the outside of the ovary corresponding to the position of each partition. In each angle formed by the converging partitions there are two rows of minute white bodies which are the ovules. This whole structure above the stamens corresponds to three fused carpels united along their adaxial surface. The fusion of the three styles and stigmas is complete. The three ovaries are still distinguishable and correspond to the three compartments of the compound or syncarpous ovary. The sides of the carpels form the partitions. The axis of the ovary, to which the ovules are attached, is formed by the fused margins of the carpels. The mid-ribs may be seen running down the outside wall, midway between the grooves.

The ovules enclosed within the compartments have very much the same structure as those of the Buttercup and contain within their nucellus a large cell, the embryo-sac (megaspore) which gives 
rise within itself to the small cœenocytic megagametophyte. As in the Buttercup it contains eight nuclei, one of which belongs to the ovum.

When the pollen-sacs dehisce and the pollen is shed each grain consists of two cells. The pollen-grains of the wild Hyacinth also are carried by insects from the anthers to the stigma. There the larger cell of the grain grows out and burrows into the tissue of

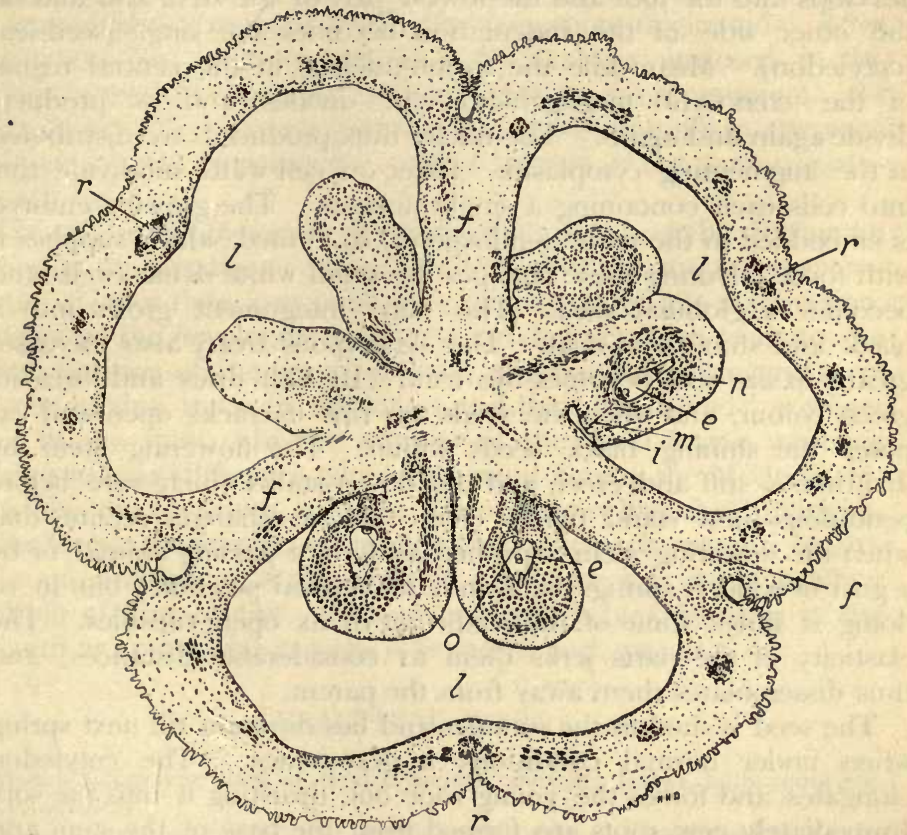

FIG. 89.-Scilla nutans, syncarpous ovary, transverse section, $\times$ зо. $e$, embryo-sac; $f$, fused sides of carpels; $i$, integument; $l$, loculus, or cavity, of ovary; $m$, micropyle; $n$, nucellus; $o$, ovum in embryo-sac; $r$, mid-rib of carpel.

the stigma and passes down through the style till it emerges in one of the compartments of the ovary. From there it grows along the inside of the compartment and makes its way to the micropyle of an ovule. It enters and transmits the two sperm-cells formed in the pollen-tube to the megagametophyte. One of these fuses with the ovum and the nucleus of the other with the fusion nucleus in the middle of the megagametophyte. 
As in the Buttercup the oosperm forms a cell-wall and by celldivision produces a short column of cells lying in the micropylar end of the embryo-sac (megagametophyte). From the two lowest cells of this column the embryo is developed. The rest of the column is the suspensor. By repeated divisions these two cells give rise to an ovoid mass of cells. A shallow indentation on one side divides the embryo into two lobes: that next the suspensor develops into the root and the lowest part of the stem and that on the other side of the indentation becomes the single seed-leaf (cotyledon). Meanwhile the fusion-nucleus in the central region of the cœnocytic megagametophyte divides and its products divide again and again. The nuclei thus produced are distributed in the augmenting cytoplasm. Later on cell-walls subdivide this into cells each containing a single nucleus. The growing embryo is embedded in the tissue (endosperm) so formed, which supplies it with food. During these changes the small white ovule swells and becomes a globular seed. The outer integument grows into a black and shiny seed-coat. The syncarpous ovary also increases greatly in size and becomes the fruit. Its wall dries and loses its green colour, and when the seeds are ripe it cracks open and exposes the shining black seeds within. The flowering stem by this time is stiff and erect, and the flower-stalks which were before pendulous now stand rigidly erect. These changes secure that when the flowering stem is pushed aside by a passing animal or by a gust of wind it springs back into its vertical position; but in so doing it slings some of the seeds out of its open capsules. The elasticity of the parts jerks them to considerable distances, and thus disseminates them away from the parent.

The seed is shed in the summer and lies dormant till next spring when under normal conditions it germinates. The cotyledon elongates and forces the young root out, thrusting it into the soil. Immediately new roots are formed from the base of the stem and fix the seedling in the soil. Just below the apex of the stem a new leaf is formed, which develops chloroplasts and carries on photosynthesis. Till this function has been initiated, the cotyledon, still retaining its top in the seed, continues to transfer nutriment to the seedling. Once independent the cotyledon atrophies and connection with the seed is severed.

From this account it appears that the life-history of the wild Hyacinth corresponds very completely with that of the Buttercup. The herbaceous sporophyte is a complex, independent, holophytic plant. In addition to its vegetative organs it produces sporophylls which are grouped together on shortened axes to form flowers. 
Spores of two kinds (microspores and megaspores) on their appropriate sporophylls are found in each flower. 'The microspores (pollen-grains) germinate as they are about to leave the microsporangia (pollen-sacs), but the gametophyte is only formed if they reach the receptive pad (stigma) on the megasporophyll (carpel). There the microgametophyte (pollen-tube) develops parasitically and transmits to the ovum of the megagametophyte its sperm. The megagametophyte also develops parasitically but without leaving the sporangium (ovule) in which the megaspore originated. After fertilisation the oosperm becomes the embryo enclosed in the megagametophyte which is still surrounded by the sporangium. As in the Buttercup this seed contains the young sporophyte, the remnants of the two gametophytes and a portion of the parent sporophyte.

The salient differences between the Hyacinth and the Buttercup consist in the arrangement and number of the parts of the sporophyte. Under these categories may be mentioned the suppression, or delay in the development, of one cotyledon, which leads to the lateral position of the growing point, the parallel arrangement of the conducting tracts in the leaves and their "scattered" arrangement in the stem. In the number of the floral leaves we have another striking difference which is general among the Monocotyledons like the Hyacinth, and the Dicotyledons like the Buttercup. In the former the number of the floral leaves in each category is usually some multiple of three, while in the latter it is more usually a multiple of four or five. Cohesion of the leaves, whether in the perianth or among the carpels, is found in both groups.

\section{PRACTICAL WORK}

Sketch a complete plant of wild Hyacinth, showing leaves, bulb, roots and flowering stem.

Make a transverse section of the flowering stem. Sketch and show the position of the conducting tracts.

Sketch a flower from above, showing the carpels, stamens and perianth.

Make a special sketch of the carpels alone.

Prepare a longitudinal section of the flower and draw it, showing the attachment of the various floral leaves.

Locate the dissolved pigment in the vacuoles of the cells of the perianth.

Make a transverse section of the ovary. Show in your sketch the three carpels and the attachment of the ovules. 


\section{LECTURE XXVIII.}

THE oosperm by repeated cell-divisions gives rise to the embryo. At first the whole embryo is composed of undifferentiated cells only, quite similar in appearance to one another. Gradually in the base of the embryonic stem and in that of the embryonic root some cells begin to elongate and to form procambial tracts. These in turn become the conducting tracts while the cells round about them become fundamental tissue and cease dividing. A similar differentiation takes place in the cotyledons. In this way the groups of cells still dividing become isolated from one another, and occupy positions at the end of the root and at the tip of the stem; and the separate growing regions of the plant are established as direct descendants from the oosperm. Hence it is that all the cells and modified combinations of cells, which constitute the mature plant, may be traced back to the subdivisions of this single cell. It may be further noted that the sporogenous cells of the sporophylls are directly traceable to the oosperm.

Each cell-division in these countless lines of cell-descent all leading back to the one common origin is preceded by the division of the cell's nucleus. Just as each cell results from the division of a pre-existing cell so the nucleus never arises de novo, but each nucleus is the result of the division of a pre-existing one. How this nuclear division is effected it is now of importance to consider.

The nucleus of a cell in the resting state, i.e. one which is not immediately about to divide, is approximately globular in form. It is limited by an extremely delicate nuclear membrane which encloses a clear nuclear fluid. Suspended in this fluid is a viscid colourless substance, which forms irregular filaments or threads, crossing the fluid and sometimes lying in contact with the membrane and anastomosing with itself, so as to form a very irregular net-work. Adhering to, or embedded in, the network are numerous granules of high refractive index, sometimes fairly uniform in size and sometimes variable in size. In many resting nuclei these granules appear to crowd the cavity of the 
nucleus so that the nucleus as a whole presents a granular appearance. Distinct from these and apparently floating free in the nuclear fluid are one or more somewhat larger granules, also of high refringency. These are called the nucleoli and are often salient objects in the resting nucleus. When such a resting nucleus is killed and stained by suitable reagents, it is found that the granules on the nuclear thread fix a basic stain readily. They probably react in this manner owing to the fact that they contain a large proportion of nucleinic acid, which is a complex organic acid possessing a high percentage of phosphorus. The substance forming these granules, owing to its readiness to fix stains, is called chromatin. The material forming the viscid thread itself does not take basic stains and is called linin. The nucleoli are also stainable, but with acid stains; and hence the chromatin granules and the nucleoli may be made to stand out plainly in preparations stained with a mixture of an acid and a basic stain. The nuclear fluid, on the other hand, does not take stain. The nuclear membrane fixes basic stains, but it is so thin that its coloration is feeble. The different behaviour of these parts towards stains has been most helpful in studying the structure of the nucleus; and with proper controls gives some indication of the chemical nature of its constituents.

When a nucleus is about to divide the bulk of the chromatingranules increases, and the anastomoses of the nuclear thread are gradually dissolved. The chromatin-granules become uniform in size and space themselves uniformly along the thread. In this state they are often called chromomeres. The thread now breaks up into a number of short lengths - the chromosomes. The nucleoli disappear and possibly add their substance to the chromosomes. The chromosomes arrange themselves in the equatorial plane of the nucleus, and form a disc usually called the equatorial plate. The number of chromosomes is usually under 100. The number is constant in the vegetative tissues of each plant. About this time the nuclear membrane breaks down, and some of the finely granular, almost homogeneous surrounding cytoplasm invades the nuclear cavity. In living cells this mass of cytoplasm appears as a highly refringent barrelshaped structure, lying in the space previously occupied by the nuclear fluid, with the chromosomes, each now bent into an L-shaped form, lying round its middle. The blunt ends of this mass terminate at the poles of the nucleus. When the cell is killed and stained this structure appears composed of a sheaf of fibres, and is called, owing to the difficulty of staining it with 
the usual nuclear dyes, the achromatic spindle. The chromosomes appear each associated with a single spindle-fibre, one limb directed towards one of the poles, while the other lies approximately in the equatorial plane extended towards the periphery. At the same time stained specimens often show stria of cytoplasm similar to the fibres of the achromatic spindle, radiating from the poles into the general granular cytoplasm of the cell. It is possible that the fibres of the achromatic spindle are produced in the killing and fixing of the protoplasm, being formed as a

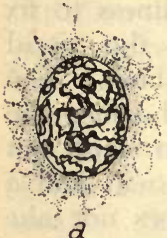

a

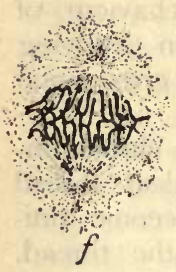

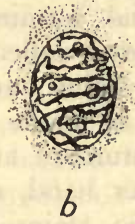

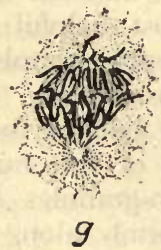

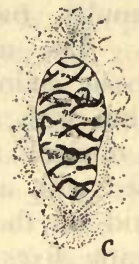

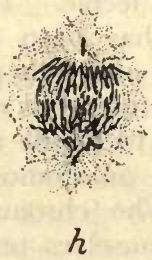

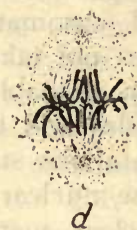

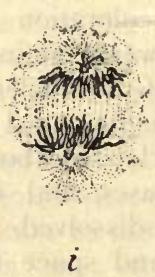

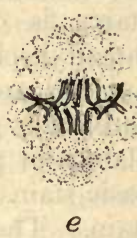

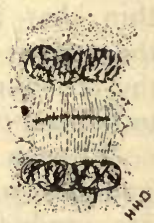

$k$

Fig. 90.-Lilium martagon, somatic mitosis, $\times 700$. $a$, nuclear thread formed from net-work; $b$, nuclear thread shortened and thickened; $c$, formation of chromosomes; $d$, equatorial (nuclear) plate of chromosomes ; $e$, longitudinal cleavage of chromosomes; $f$, beginning of the separation of the half chromosomes; $g$, parting of the halves; $h$, movement of the halves towards the poles; $i$, congregation round the poles; $k$, formation of the new nuclei from the halves and appearance of the cell-plate across the middle of the achromatic spindle.

coagulation product between the colloids of the central protoplasm and the reagents used. This surmise is rendered more probable by the fact that similar structures may be produced in egg-white with the usual fixing reagents.

The next change observable is the splitting of each chromosome longitudinally. In suitably stained specimens it may be seen that this cleavage is the result of the division of each of the minute chromomeres of the thread. The two parallel linear series formed by this division constitute the halves of the chromosomes. These 
halves part asunder and moving along their associated fibres migrate to the opposite poles. Round the poles the half chromosomes congregate and draw together to form a dense mass of chromatin and linin.

While the halves of the bisected chromosomes are moving apart, at the middle of each fibre of the achromatic spindle, a bright refringent speck appears. It is composed of a cellulose-like substance called pectose. The specks of pectose together form the cellplate. They gradually grow in size and finally coalesce to form a disc, still apparently perforated by fine filaments of protoplasm -the persisting remains of the achromatic fibres. Subsequently layers of cellulose are deposited on each side of the cell-plate and are extended out from it to unite with the lateral walls of the cell, thus dividing it into two. While this partition-wall is being constructed the masses of chromosomes at the poles become more regular by the drawing in of those limbs of the chromosomes which were still projecting into the cytoplasm, or were delayed in their motion along the fibres. As soon as, or even before, the surface of the mass is smoothened by this means, vacuoles or cavities appear in its substance. Vacuolation continues, the partitions dividing the vacuoles become reduced to filaments and only an extremely delicate pellicle remains to separate the coalescing vacuoles from the surrounding cytoplasm. Nucleoli reappear and the two nuclei resulting from the division of the original one are completely reconstituted. By this time the new cellwall is complete and the new cells, each with its own nucleus, have been produced from the original single cell. Before the next division of the new cells their nuclei will divide again in the manner just described.

The process of nuclear division which I have just outlined is called mitosis or karyokinesis. In it the following stages are for convenience of description usually recognised: (I) The spiremestage, when the nuclear thread is freed of its anastomoses and forms a continuous skein in the nuclear cavity; (2) the equatorial plate, nuclear plate or monaster stage, when the chromosomes lie in the equatorial plane of the nucleus and appear as a stellate mass when viewed from the poles; (3) the diaster stage, when the longitudinal halves of the original chromosomes, having parted asunder, come to form two stellate masses round the poles; (4) the dispireme stage, when the nuclear net-work is reformed in the two resulting nuclei.

Evidently by the process of mitosis the ever-growing material of the nucleus of the oosperm is allotted equally to all the cells of the 
resulting plant. But the complicated procedure not only secures that the bulk of nuclear material received by each cell shall be equal, but by the fission of the individual chromomeres it provides that the nuclei of every cell of the mature plant will contain a representative of every chromomere of the nucleus of the oosperm. Hence, even if the chromomeres differ among one another, mitosis secures that the resulting nuclei shall be equivalent to one another, not only quantitatively but also qualitatively.

The elaborate mechanism adopted for the accurate division of the nuclear substance naturally arouses speculation and forces us to consider what the nature of this substance must be which calls for such meticulous distribution throughout the cells of the organism. The cleavage and distribution of the chromosomes or rather of the chromomeres seem to be the essential result aimed at in mitosis, and any satisfactory hypothesis must take this fact into account. The generally received view is that the chromatin is the hereditary substance, or germ-plasm, of the organism. It controls and directs the development of the cells and secures that they shall have the characteristics of the species to which the individual belongs, and shall contain representatives of the distinctive traits, and features which characterised that individual's parents. The separate characteristics and traits, or groups of them, are probably represented by portions of this substance, viz. the chromosomes and chromomeres. Thus the chromatin of the oosperm being distributed throughout the cells of the embryo compels the development of the latter into an organism like its parents. This hypothesis fits in well with the observation that, given the necessary conditions, each and every cell of a plant or animal appears able to give rise to a complete organism, containing as it does in its nucleus the complete germ-plasm. The propagation of plants by buds, cuttings and offsets furnishes familiar examples of this phenomenon.

When the oosperm is formed by the fusion of the sperm and ovum, it receives the nuclei of two individuals, and consequently the individual produced must, if our theory be correct, combine the characters of the two. This surmise is justified by observation. Furthermore, the fact that the offspring resembles the male parent as much as the female, constitutes one of the most cogent arguments that the substance of the nucleus is the germ-plasm. For the sperm contributes little else than chromatin, and the bulk of its chromatin is approximately if not exactly equal to the bulk of the chromatin contained by the ovum.

The hypothesis further necessitates that the oosperm must con- 
tain double the amount of germ-plasm contributed by each gamete and as the number of chromosomes in each mitosis is equal to that of the preceding one it follows that the amount of the germplasm and the number of the chromosomes of the oosperm are double those of each gamete. This consideration shows, if our conclusions are correct, that there must be some point in the line of cell divisions between the successive acts of fertilisation in the sequent generations, where a reduction in the number of chromosomes, and with it a reduction in the number of transmitted characters, occurs. It is of peculiar interest that this reduction was established only after its occurrence had been predicted on theoretical grounds.

In the higher plants the reduction-mitosis usually takes place during the division of the sporogenous cells or the spore-mothercells. The large nucleus of these cells when preparing itself for mitosis develops a particularly long and attenuated nuclear thread. The length of this thread is very great, and it forms countless intricate coils in the nuclear cavity. Gradually these coils resolve themselves into a number of loops twisted more or less upon themselves. At this stage a curious and sudden change comes over the nucleus. The thread, which has been up to this more or less uniformly distributed through the nuclear cavity, is now swept across it and forms a dense and tangled mass at one side, in close contact with the large nucleolus. This stage, which is called synapsis, lasts but a short time, and then the mass unravels gradually and the whole thread emerges in twisted loops. There is some uncertainty whether the loops formed before synapsis are identical with those which emerge after it. It is possible that the thread forming the emerging loops is composed of two parts of the original thread and that each emerging loop is thus fourfold of the original thread. However this may be, the emerging thread breaks into a number of twisted portions equal to half the number of the chromosomes of the somatic cells of the plant ; e.g. if the nuclei of the somatic cells contain twenty-four chromosomes, in this mitosis twelve chromosomes only appear. Thus each of these is a double chromosome, containing a double number of chromomeres. After the separation of the chromosomes growth in thickness continues, apparently at the expense of the nucleoli which now disappear. When the chromosomes have so far thickened that their width is about half to a quarter of their length, the nuclear membrane disappears and its cavity is invaded by the cytoplasm. The achromatic spindle is formed and the short double chromosomes, having the form of pairs of twisted rods, loops or rings, 
arrange themselves in the nuclear plate. Cleavage by separation of the adjacent chromomeres occurs here and the halves of each double chromosome pass along the spindle to the opposite poles. The halves as they leave the nuclear plate usually have a V-form, and by the way they disentangle themselves from one another, they seem to show that each limb of the $\mathrm{V}$ is composed of half one of the twisted rods of a double chromosome. As the V-shaped chromosomes reach the poles they tend to break up into their component chromomeres, and becoming surrounded by a nuclear membrane they constitute a new and highly granular nucleus.
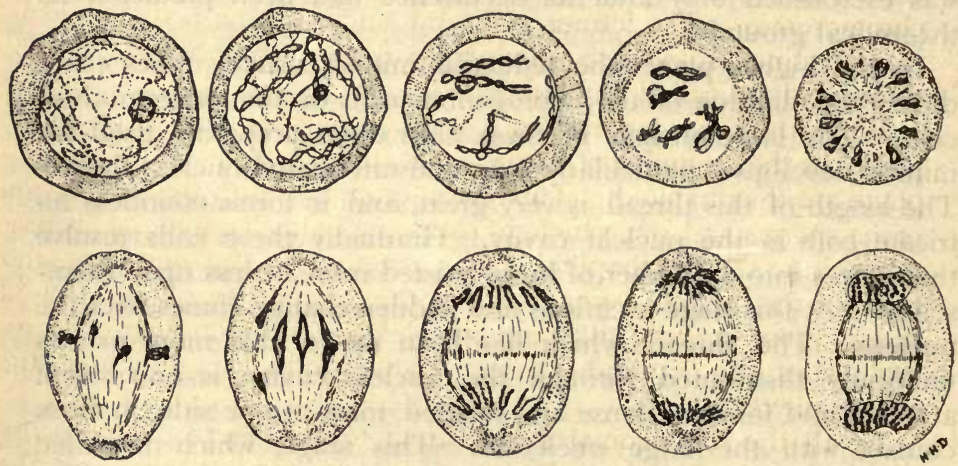

FIG. 9r. - Lilium martagon, reducing mitosis in pollen-mother-cell, $\times 700$. Stages shown consecutively from left to right. In upper row: $\mathbf{r}$, long nuclear thread formed from net-work; 2, nuclear thread twisted on itself; 3 , chromosomes formed from twisted loops of nuclear thread; 4, chromosomes shortened and thickened; 5, nuclear membrane has disappeared, cytoplasm has invaded the nuclear cavity and the chromosomes are arranged in the equatorial plane, polar view. In lower row: I, same stage as 5 above, seen from the equatorial plane; 2 , separation of half chromosomes; 3 , convergence of half chromosomes to the poles, de. velopment of cell-plate; 4 chromosomes break up into granules at poles; 5 , formation of new nuclei from these granules.

While the exact manner, in which the cleavage of the chromosomes in the equatorial plate takes place, rests in doubt, the essential differences between this reduction-mitosis and the ordinary somatic mitosis are plain: in the reduction-mitosis the number of chromosomes differentiated from the nuclear thread and the number contributed to each resulting nucleus is half that found in the mitoses leading up to the formation of the sporogenous cells and in those of the surrounding somatic cells. Furthermore, the chromomeres distributed to the new nuclei probably do not, as in the somatic 
mitoses, represent halves of chromomeres of the original thread, but are these segments themselves intact. Hence the chromomeres in the resulting nuclei may not be equivalent.

The two nuclei formed by the reduction-mitosis immediately undergo a second division. This mitosis conforms to the ordinary procedure of somatic nuclear division, except that in the differentiation of the chromosomes the reduced number of chromosomes appears. The nuclei then of the second mitosis of the sporogenous cells contain this reduced number, or if we indicate the number of chromosomes in the somatic cells as $2 x$ these nuclei, which, as we know, become the nuclei of the spores, contain $x$ chromosomes. The former cells and nuclei are termed diploid in contradistinction to those which contain the $x$ number of chromosomes and are called haploid.

When the spore germinates, a series of mitoses is initiated which preserves the $x$ number of chromosomes and the cells of the gametophyte are thus haploid. This series terminates with the production of the sperms and ova which, of course, are also haploid. Fertilisation brings the two haploid nuclei together in the oosperm, and initiates the diploid series which is the sporophyte. The diploid phase then persists till reduction in the spore-mother-cells reestablishes the haploid phase, which continues throughout the gametophyte.

In all the Archegoniates and in the Seed-Plants the reducing division normally takes place in the spore-mother-cells, and the tetrad cell-division characteristic of spore-formation is thus associated with the reducing mitosis which initiates the haploid phase. The cell-division producing the megaspore of Seed-Plants is never a characteristic tetrad division-the four cells resulting form a linear series; and it is just in connection with the production of the megaspores that an irregularity in the occurrence of the reducing mitosis has been found. Instead of the reducing division occurring in the mother-cell, it is often postponed till the first division of the megaspore (embryo-sac).

In Polysiphonia as we might expect from the formation of the tetraspores reduction takes place at their origin, and the gametophyte resulting from their germination is haploid. Haploid nuclei characterise its gametes. The oosperm is evidently diploid and produces by its nuclear and cell-divisions the diploid sporophyte, which by a reducing mitosis produces the haploid tetraspores. So the two phases correspond with the limits of the alternating sporophyte and gametophyte.

Evidently a different state of affairs must obtain in a plant like 
Fucus, in which there is no spore-formation in the life-history. In it reduction has been found to take place in the antheridium and in the oogonium; so that while the somatic cells of the plant are diploid; this reduction secures that the gametes are haploid. The diploid condition is restored by fertilisation. It is of interest to note that in the position of the reducing mitosis Fucus conforms to the animal scheme.

In other plants such as Spirogyra reduction takes place in the oosperm.

These instances suffice to establish the general truth of the proposition that in those plants whose life-history includes the production of gametes and fertilisation a reduction-mitosis is always found. The position of the reduction-mitosis in the lifecycle varies in different groups.

\section{PRACTICAL WORK.}

Fix young buds from the flowering stem of a Turk's Cap Lily-Lilium martagon -in a large volume of methylated spirit. Leave them to harden for 24 hours.

Cut transverse sections of buds about 5 millimetres and less in diameter. Float the sections in water and select out the best and transfer them into some very dilute Delafield's hæmatoxylin. Stain for r8-24 hours. Wash the sections in a change of water and pass them through three changes of spirit into oil of cloves. When the sections are quite clear lift them from the oil of cloves with a needle, gently touch them against a piece of paper, to remove the excess of oil, and transfer into a drop of balsam on a slide and cover.

While cutting the sections observe the six overlapping parts of the perianth and the arrangement of the six stamens round the three fused carpels. In the larger buds the yellow mass of pollen in the pollen-sacs is plainly visible, and the fused carpels form a triangular prism in the centre of the bud. The sections of this will show the three compartments of the syncarpous ovary. In some of these sections will be seen ovules. Where the section passes through the axis of an ovule the embryo-sac (megaspore) will be exposed to view. If by good fortune the section passes through an embryo-sac during the first mitosis, a stage of the reducing division will be shown. Somatic mitoses will probably be found in the nucellus and in the walls of the ovary. If the selection of the buds has been fortunate reducing mitoses may be found in the pollen-mother-cells in the young anthers. Any mitosis observed should be carefully sketched.

Another very good source of material in which mitosis may be studied is the developing seeds of the Crown Imperial Lily, Fritillaria imperialis. Open the erect green fruits of this lily and take out the immature seeds when they are about 5 millimetres long. Put them in methylated spirit, having previously pricked the swollen central part with a needle to allow the spirit to penetrate quickly. After $18-24$ hours the seeds may be split in two, thus opening up the enlarged embryo-sac. Remove with a needle the protoplasmic lining of the sac, wash, stain, dehydrate with spirit, clear in oil of cloves and mount in balsam. In the best preparations the lining of the sac is a thin 
pellicle with large nuclei distributed in it. No cell-walls have yet been formed and the nuclei are often seen in mitosis. The stimulus for division seems in these cases to advance as a wave across the pellicle so that successive rows of nuclei in the successive stages of mitosis may be observed.

As these preparations are largely a matter of good fortune in hitting off the exact stage in development, many preparations should be made. A successful preparation will more than repay the trouble, and affords a record of the minutest details of perhaps the most interesting and suggestive biological phenomenon.

Even the thicker pellicles, in which cell-division has already taken place, will afford preparations showing many mitoses. In these the details of the process may often be seen with the most astonishing clearness. 


\section{LECTURE XXIX.}

THE similarity between parents and offspring, though often very great, is never exact. There are always greater or smaller differences observable. The similarity is usually very striking when the two parents resemble one another very closely and come from the same stock, and greatest of all when the contributing gametes are, as in the case of some organisms (e.g. many of the Seed-Plants) derived from the same individual. But even in these cases certain differences are found. Some of these may be undoubtedly traced to the action of the surrounding conditions on the developing oosperm. Thus starvation may make the offspring of a vigorous parent puny ; and its dwarfishness and its unhealthiness cannot be said to be characteristics transmitted to it from its parent, but are acquired from its conditions. Experimentally we may show divergences brought about by differences in surroundings by taking a number of seeds, formed when the pollen of a flower falls on its own stigma, and sowing them under different conditions. The seedlings which grow up in darkness are drawn and attenuated, those which develop in absence of iron salts or of light are without green colour, those which are surrounded with an excess of salinity become more fleshy, those which lack various requirements are weak and puny. Presumably each seed inherited very similar qualities, but they acquire very different characteristics from the action of their environment. That the majority of their characteristics are inherited can scarcely be doubted, for no matter how different the conditions of growth are, if life is at all possible, all the plants are recognisable as being of the same kind as the parent. This specific similarity cannot be attributed solely to the similarity of conditions of nurture surrounding the early stages of the embryo, for it is quite as marked in the lower plants where the oosperms and spores begin their development outside the body of the parent.

Hence we recognise that the characters of each individual are in part inherited and in part acquired. It is interesting to note that in spite of a very general popular belief to the contrary no clear instance has been established of the transmission of such 
acquired characters. ${ }^{1}$ Thus so far as accurate observation only is relied on, no character, be it a deformity or an "acquirement," which is directly derived from the action of the environment, has been shown to be transmitted from the parent to its offspring. Hence it is generally believed that the surroundings are without direct influence on the germ-plasm or hereditary substance.

The study of heredity has attracted attention from the earliest times not only owing to its great intrinsic interest as one of the most inspiring secrets of nature, but also owing to its manifestly great practical importance. Despite vast numbers of experiments and speculations very little advance was made towards discovering its mechanism, or the laws of its action, till the middle of the last century. Even then, when a most important clue was discovered, it lay long unrecognised; so that this clue has only been generally utilised as an instrument of research since the beginning of the present century.

The clue was furnished by Mendel in I866. He carried out his pioneer experiments with Seed-Plants, and in point of fact with the edible pea. The facts, that the stamens containing the pollen and the carpels containing the ovules occur in the same flower, that self-fertilisation normally takes place if the flowers are not interfered with, and also that the stamens are easily removed without injury to the carpels, were great advantages in this work. It was thus possible at will to procure offspring from one parent alone, or, with equal certainty, from any two given parents.

Mendel experimented with plants which, while generally resembling each other, differed from one another in possessing either one or the other of a pair of differentiating characters (allelomorphs). The object of his experiment was to observe how these characters combine in the hybrid formed, and to deduce the laws according to which they appear in the successive generations. For example, some peas are round in contour, while others have somewhat flattened faces upon them, and are angular or quadrate in form. These properties are characteristic of certain varieties, and it is easy to obtain strains which reproduce these differences regularly in succeeding generations. In this case rotundity and angularity form a pair of differentiating (mutually exclusive) characters. Characters which may be thus paired in contrasted individuals are called allelomorphs. It should be noted that, while these two allelomorphs (roundness and angularity) are assigned to the seed,

${ }^{1}$ An important exception to this general rule may possibly be brought to light by the line of work recently operred up. 
they are in reality characters of the embryo enclosed in the seed.

At first the seeds were tested by sowing the two kinds apart. When mature plants were formed they were automatically selfpollinated. At the same time Mendel guarded by special precautions against other pollen reaching the stigmas. Plants which are thus pollinated with their own pollen are said to be "selfed," while those which receive the pollen from another plant are described as cross-pollinated or crossed.

When the embryos in successive generations produced by selfing always showed the same allelomorph, whether it was roundness or angularity, it was judged that so far as this character was concerned the peas selected could transmit either allelomorph only.

Then the experiment was tried of cross-pollinating a plant developed from one of the round peas with the pollen from one

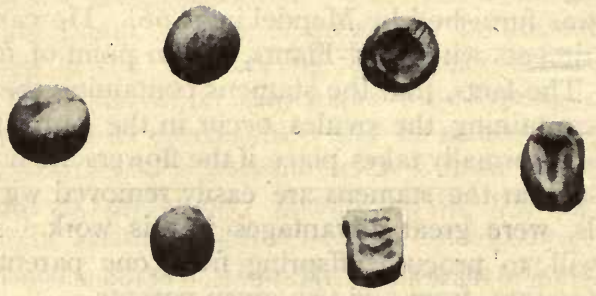

FIG. 92.- Round and angular (or quadrate) peas.

coming from an angular pea. The reciprocal cross was also made, i.e. the pollen of an angular plant was put on the stigma of a round plant. The result was the same from both crosses. Whether the round plant was used as the pollen-parent or the seed-parent, the embryos produced in the pod of the seed-parent were always found to be round. Mendel called the character which thus appeared in the hybrid to the exclusion of the other the dominant character, while that which was not visible he styled the recessive character. In this case he found that roundness is dominant and angularity recessive, no matter which way the cross is made. It may be noted that this indicates that the inheritance of the offspring is equally contributed to by each parent. The first or hybrid generation is called the $F_{1}$ generation.

The flowers of the mature plants developed from these round peas produced by cross-pollination were selfed. Embryos $\left(\mathrm{F}_{2}\right.$ generation) were developed in the selfed carpels and they were 
found to be of two kinds, viz. round and angular. When a large crop of these was harvested it was found that always approximately 25 per cent. of the crop was composed of angular embryos and 75 per cent. of round embryos. That is, in the $\mathrm{F}_{2}$ generation threequarters of the total number of embryos exhibit the dominant character and one-quarter exhibits the recessive one. When the plants from all the seeds of the $\mathrm{F}_{2}$ generation mature and are selfed, an interesting difference is found among those developed from the rounded embryos, one-third of these produce rounded embryos only, while in the pods of two-thirds both round and angular embryos are found. In these pods the proportion is 3 round to I angular. In the pods of the selfed plants derived from the angular embryos no round embryos are to be found.

The experiment may be summarised by the following diagram, where the plants derived from the round seeds are represented as circles and those derived from the angular seeds are represented

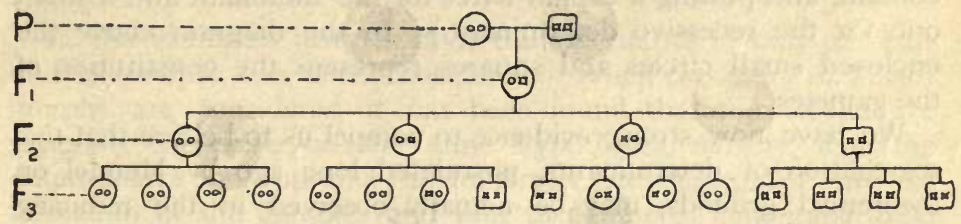

FIG. 93.-Diagram to illustrate the transmission of roundness and angularity in three generations. The small enclosed figures indicate the determinants in the germ-cells.

by quadrate outlines. In point of fact in the embryonic state the plants are round and angular respectively.

It should be particularly noticed that individuals may be found in the first generation after a hybrid $\left(\mathrm{F}_{2}\right)$ which breed true respecting either allelomorph. Thus in the $F_{2}$ some of the round individuals on being selfed will produce only round offspring, and similarly all the angular on being selfed produce only angular offspring. It follows that, with regard to the differentiating characters of a hybrid union, $5^{\circ}$ per cent. of the offspring are pure. Of this 50 per cent., half transmit one allelomorph and half transmit the other.

These observations Mendel explained as follows: The original two lots of tested seeds grew into plants which produced gametes containing in their germ-plasm determinants (factors) of roundness and angularity respectively. When crossed an oosperm was formed in which determinants of roundness and angularity were contributed 
by the respective parents. In the cell-divisions which convert the oosperm into the embryo each resulting cell has both kinds of determinants distributed to it ; but inasmuch as the determinant of roundness is dominant the embryo develops round. Originating from gametes carrying alternative determinants this individual is called a "Heterozygote". To account for the observed offspring of this hybrid plant, Mendel assumed that at the formation of the gametes a separation and sorting of the determinants of the pairs of allelomorphs take place, so that each gamete receives the determinant of roundness or the determinant of angularity, but no gamete ever receives the two determinants of a pair. Since large numbers of gametes are formed and there is an equal number of those containing the determinant of roundness as of those with that of angularity, it is evident that there is an equal chance for the unions $\mathrm{RR}, \mathrm{R} a, a \mathrm{R}$, and $a a$ (indicating the gametes by the initial letter of the allelomorph, the determinant of which they contain, and putting a capital letter for the dominant and a small one for the recessive determinant.) In the diagram above the enclosed small circles and squares represent the constitution of the gametes.

We have now strong evidence to compel us to believe that this segregation of determinants, postulated long ago by Mendel on theoretical grounds, may be actually observed in the reducing mitosis of the spore-mother-cells and in other tetrad mitoses.

The characters of roundness and angularity in the embryo are only one pair of many allelomorphic pairs in peas investigated by Mendel. Another one was also a character of the embryo and recognisable as soon as the seed is mature. It was that of greenness and yellowness. In some varieties the embryo is green in the ripe seed, while in others it is yellow. Mendel showed that this character is transmitted according to exactly the same scheme as that which defines the transmission of roundness and angularity. In this case yellowness is dominant and greenness is recessive. So that the hybrid formed is yellow and when self-pollinated it bears 75 per cent. yellow and 25 per cent. green embryos. As before when the individuals of this $\mathrm{F}_{2}$ generation are self-pollinated it is found that one third of the yellow produce nothing but yellow embryos, and two thirds of the yellow have a mixed progeny. The green when selfed produce green only.

In these two examples complete dominance of one determinant over the other of the pair is illustrated. This complete dominance appears to be the general rule. However, some cases are known where the heterozygote, or hybrid, resulting from the fusion of two 
gametes each contributing the alternate determinants, does not possess either allelomorph but has an intermediate character. The classical example of this is furnished by the blue colour of the Andalusian fowl. When blue Andalusian fowl are paired together only half of the chickens produced become blues, the remainder are "wasters," black and dirty whites in about equal proportions. Repeated efforts have been made to breed a pure progeny from blue parents without success. Assuming that the blues are heterozygotes this is what might be expected. This surmise has been established by producing whole progenies of blues without any wasters by pairing the black and white wasters.

Similar heterozygous forms are produced by plants. Thus the peculiar shade of flower in Primulas known as "crushed strawberry" cannot exist except as a heterozygous character. It is formed by the united action of the determinants of a pair of allelomorphs, of which one is deep crimson and the other white.

In the examples just described the inheritance of only one pair of allelomorphs has been considered. When two pairs of allelomorphs are considered it has been found that the. results are generally explicable on the same hypotheses, namely, that the determinants of opposing allelomorphs are segregated at the formation of the gametes and that the gametes containing these different determinants are formed in equal numbers and unite together indiscriminately. It is further premised that the various determinants are transmitted without influence on, and independently of, one another.

Using, for the sake of convenience, the shape and colour allelomorphs of the peas for our illustration, we will consider the inheritance of these allelomorphs among the descendants of two pure peas, one round and green and the other angular and yellow.

Here the pairs of allelomorphs are roundness, angularity, and yellowness, greenness. The dominants are roundness and yellowness; angularity and greenness are the recessives. We will denote the determinants of the two pairs of allelomorphs as R, $a$ and $\mathrm{Y}, g$.

The gametes of the round and green pea will all onntain $\mathrm{R}$ and $g$ the determinants of their characters. The gametes of the angular and yellow pea will similarly all contain $a$ and $\mathrm{Y}$. In fertilisation the heterozygote $\mathrm{R} g a \mathrm{Y}$ will be formed. $\mathrm{R}$ and $\mathrm{Y}$ being dominant this heterozygote will appear round and yellow.

When this heterozygous embryo grows up, segregation of the 
determinants will occur in the spore-mother-cells preliminary to the formation of the gametes (sperms and ova). Four different kinds of sperms will be found, viz. $\mathrm{RY}, \mathrm{R} g, a \mathrm{Y}, a g$ in equal numbers; and four different kinds of ova, viz. $\mathrm{RY}, \mathrm{Rg}, \dot{a} \mathrm{Y}, a g$, also in equal numbers. The four different kinds of sperms will unite indiscriminately with the four different kinds of ova, so that sixteen distinct combinations or zygotes (oosperms) will result: RYRY, $\mathrm{RYR}, \mathrm{RYaY}, \mathrm{RYag}, \mathrm{R} g \mathrm{RY}, \mathrm{R} g \mathrm{R} g, \mathrm{R} g a \mathrm{Y}, \mathrm{R} g a g, a \mathrm{YRY}, a \mathrm{YR}$, $a \mathrm{YaY}, a \mathrm{Yag}, a g \mathrm{RY}, a g \mathrm{R}$, aga $\mathrm{Y}$, agag.

These combinations will be more easily followed by reference to the accompanying diagram. Here along the upper side are shown the four different types of sperms which can unite with the four possible types of ova. The latter are indicated along the left side of the square. The zygotes formed by these unions are shown in the subdivisions of the square, the determinants contributed by the sperms being in each case uppermost. The shape of the embryo is indicated by a zone round the determinants in the subdivision, and its colour by the marking of the enclosing zone. In the figure dotting indicates yellow and green is represented by short lines.

Among these zygotes RYRY, RYRg, RYaY, RYag, RgRY, $\mathrm{R} g a \mathrm{Y}, a \mathrm{YRY}, a \mathrm{YR}, a g \mathrm{RY}$, nine in all, will resemble one another and will show the characters of roundness and yellowness; the zygotes $\mathrm{R} g \mathrm{R} g$, $\mathrm{R} g a g$, $a g \mathrm{R} g$, three in number, will appear round and green ; the zygotes $a \mathrm{YaY}, a \mathrm{Yag}, a g a \mathrm{Y}$, three in number, will appear angular and yellow. There is only one agag which will appear angular and green, possessing the two recessives only. As each of these combinations is equally likely to be formed, in a large number of descendants we will get the ratios of these four different categories to one another expressed as follows : $9: 3: 3: \mathrm{I}$.

It will be evident that certain of these zygotes produced by the union of two gametes having an identical constitution so far as the characters considered are concerned when selfed must breed true, viz. RYRY, $\mathrm{R} g \mathrm{R} g, a \mathrm{YaY}$, agag. Of these "homozygotes" the first and the last, called respectively the double dominant and double recessive are new combinations and differ from both of the original parents. Thus we see how new constant varieties may arise from hybridisation.

Recent research has shown that in all probability while the dominant character is due to the presence of a determinant in the individual, the absence of this determinant causes the corresponding recessive character. Thus the recessive angularity of a quadrate pea is the result of the absence of the determinant of roundness 
which if it were present would produce roundness. Similarly a green pea owes its greenness to the absence of the determinant which if it were present would change its colour to yellow.

Mendel investigated the inheritance of a number of these allelomorphs, and he showed that like those, which I have just described, they were each transmitted independently. In this way we come to look at an individual as a mass of unit characters each represented

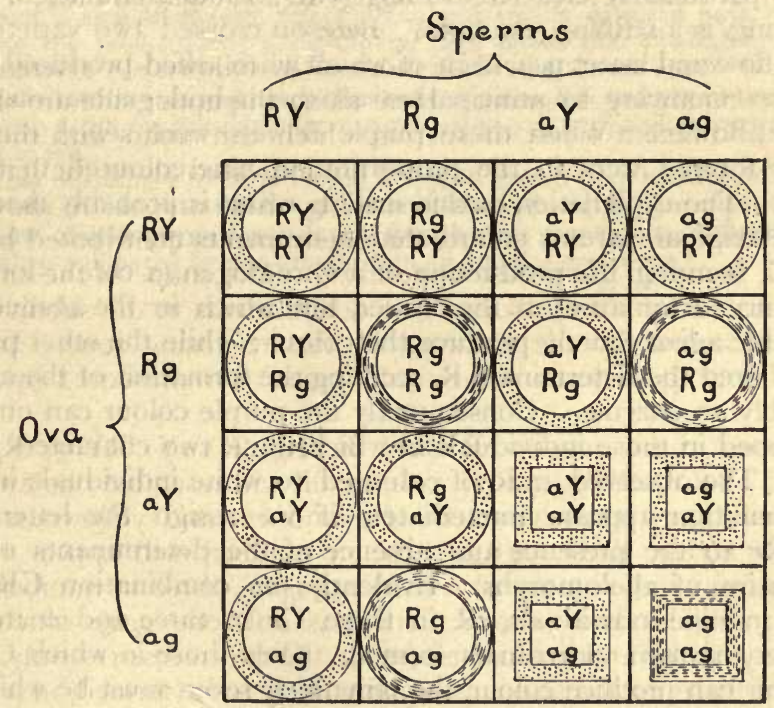

FIG. 94.-Diagram to illustrate the inheritance of two pairs of characters (Roundness-angularity, Yellowness-greenness) in the $F_{2}$ generation. The nature of the zygotes is represented by the figures in the sixteen squares. The letters indicate the determinants contributed by the germcells, those from the sperms being above in each case. The shape of the zygote produced is shown by the outline (round or angular as the case may be): when yellow, it is marked with dots and when green, with lines.

by a determinant in the germ-plasm. And evidence has since been adduced in favour of the view that the chromatin of the nucleus contains these determinants. When both parents of an individual contribute the determinant of a given character every gamete produced by that individual will receive that determinant; but where only one parent contributes the determinant then only half the gametes of the individual will receive and contain it. The 
allocation of the various determinants seems in the great majority of cases to be quite fortuitous and they do not as a rule appear to exercise any attraction or repulsion on one another tending to favour special combinations.

While the determinants are thus generally quite independent of one another the characters connected with the determinants may influence each other to a greater or less extent. This is sometimes particularly clear in dealing with colour-characters. The following is a striking example. Bateson crossed two varieties of white-flowered sweet pea, each of which when selfed produced only whites. Contrary to anticipation all the hybrid generation bore purple flowers. When these purple flowers were selfed the offspring formed were in the proportion of nine coloured to seven white. The explanation of this striking result is probably that one of the original parents contributed a determinant, which we may call C, securing the production of a chromogen (a colour-forming substance or mixture) in the flower, but which in the absence of a suitable agent cannot produce the colour; while the other parent contributed the determinant $\mathrm{R}$, securing the formation of the agent, probably an enzyme. Consequently the purple colour can only be developed in those individuals in which these two characters coincide. The observed ratio of coloured to white individuals in the $\mathrm{F}_{2}$ generation appears immediately if we assign the letters $\mathrm{Cc}$ and $\mathrm{Rr}$ to the presence and absence of the determinants of the two pairs of allelomorphs. Evidently the combination CR will occur in nine individuals, $c \mathrm{R}$ in three, $\mathrm{Cr}$ in three and $c r$ in one of every sixteen individuals formed. Only those in which $\mathrm{C}$ and $\mathrm{R}$ occur can produce colour, the remaining seven must be white.

Many cases of this interaction of characters are known, some much more complicated than that just described-characters of structure may even interact with characters of colour. Their investigation has shown how a number of apparent exceptions and contradictions may be explained.

The few instances which time has allowed to be brought forward in illustrating these fundamental laws of heredity have been nearly all drawn from experiments with plants. It must not be thought that these laws apply to plants only. Vast numbers of experiments and observations have shown that they are equally applicable throughout the animal kingdom, and may be illustrated by many instances in man himself. However, this part of the subject, although it opens up studies and speculations of extraordinary interest, is outside our province. 


\section{PRACTICAL WORK.}

Evidently breeding experiments are impossible in a short elementary course, so that we will have to content ourselves by becoming familiar with one or two classical examples of unit characters.

Select out of a mixture of edible pea seeds some which are angular (quadrate) and some which are round. If such a mixture is placed on a smooth piece of board and the latter is slightly inclined gentle shaking will make the round peas run down the slope leaving the angular ones in position on the board.

From a similar mixture select green and yellow coloured peas. By removing the seed-coat of some of the peas notice that the colour of the cotyledons of the embryo may be concealed by that of the coat. It may be recalled that the coat and the cotyledons belong to different generations.

Write down the possible offspring of a round yellow pea of unknown origin when selfed.

What offspring may be produced by plants grown from each of the following when selfed : round green, angular yellow and angular green peas?

What offspring would you expect from a plant grown from a round green pea when pollinated by one grown from an angular green pea? 


\section{LEC'TURE XXX.}

CASUAL observation of living nature and the more careful study of a certain number of plants or animals cannot fail to raise the question as to how the innumerable different kinds of organisms came into existence. Of flowering plants alone there are more than one hundred thousand species known. Of the lower plants probably nearly as many are known, and it is certain that great numbers are still to be discovered.

How did all these species originate?

In early times the answer was given with no hesitation. Each species was created at some period in the past. More recently the . further dogma was added, that species are immutable, i.e. are incapable of alteration through the lapse of ages.

Finding similarities between various animals, independent minds on more than one occasion, even in ancient times, suggested a relationship between these similar forms, and more or less clearly indicated that similar animals are similar, because they are descended from a common stock. It was, however, reserved for Darwin in the middle of the last century to devote himself to collecting and sifting evidence with the set and definite purpose of establishing or refuting this view. The evidence which he amassed and examined led him to accept and formulate the theory of Evolution.

The theory is of such universal application in biology and it involves such far-reaching deductions that biologists of all kinds have investigated its truth and adequacy from the most various points of view, and have tested it by countless observations in the most diverse branches of biology. Its sufficiency has been tried not only with the facts it was formulated to explain, but also with many others discovered since its enunciation. Besides this scientific enquiry it has been subjected to the most unsparing criticism of those who from previous training or prejudice were hostile to the theory. This controversy, in addition to sifting the evidence for and against evolution, has been responsible for a vast amount of research, and so has resulted in great additions to knowledge and has opened up avenues to the most elevating human speculations. 
It now remains to summarise very briefly the salient points of the botanical evidence which has helped to lead scientific opinion of to-day to accept the theory of the Origin of Species by Descent, or as it is often called the theory of Evolution.

We will consider the evidence under three heads : (I) Evidence from Morphology. (2) Evidence from Distribution. (3) Evidence from the Geological Record.

First with regard to morphological evidence.

(a) Argument from Classification. Even when the dogma of the immutability of species was generally held, naturalists in examining and recording the characters of species recognised that groups of these species had features in common, and were distinguished by these features. The smaller groups of species were called genera. Thus the genus Ranunculus is a group of several different kinds of buttercup, e.g. the species $R$. bulbosus, $R$. repens, etc. It was noticed that the species naturally grouped themselves so that those of one genus resembled one another more closely than the species of different genera. Similarly these genera could be arranged in larger groups, often called families. These two terms are in themselves significant. They indicate that those who applied them had in their minds the idea of racial and family relationship. The families were associated into orders and the orders into still larger assemblages called classes. In arranging the various kinds of plants and animals in the classification of species, genera, families, orders, classes, etc., the relationship of the various species in a genus, or of the genera in a family was purely ideal, and the grouping of similar forms under certain names was purely formal and was merely a matter of convenience in the minds of those who believed that species are immutable. Evidently if the different species had been formed as special acts of creation and were capable of no subsequent transformation into other species, no genetic connection linking individual species was possible. Thus classification was regarded as arbitrary, depending solely on the distinguishing criteria selected. The unsatisfactoriness of this view, coupled with the observation that family resemblances in man are based on genetic relationships and the great probability (as was strongly held by many who asserted the immutability of species) that racial characters indicate common ancestry, led biologists to reject the theory of special creation. They saw in the possibility of the classification of organisms a strong reason for assuming common descent for those species which closely resembled one another, and for believing that the closeness of resemblance is in general a measure of the closeness of the forms 
considered to the common stock. Thus the relationships revealed by classification are really genetic relationships and the different species included in a genus are the terminations of different lines of descent from a common ancestral stock. Evidently the application of the theory cannot stop here. The possibility of grouping genera into families and families in turn into orders forces us, on the same principle, to admit common origins for these groups, and we must regard the relationship connecting the living species of organisms as comparable to a vast and most complex genealogical tree, starting the pedigree with those forms of life not distinguished as belonging to either the vegetable or animal kingdom, and branching, with the growth of complexity and differentiation, into kingdoms, sub-kingdoms, divisions, classes, orders, families, genera and species. Thus each species, fitting as it does into its place among the outermost branches of this genealogical tree and indicating by its structure its relationships to its neighbours, adds evidence in favour of the theory of descent.

(b) Argument from modified parts. Naturalists are familiar with examples both in plants and animals of parts which, appearing in two different species and presenting underlying and fundamental similarities, are yet strikingly modified in shape and structure: For instance, while the leaves of many leguminous plants are subdivided into small leaf-like pinnae, in others some of the pinnae are represented by filiform tendrils, which by twining round supports sustain the weight of the plant. Such tendrils have long been described as modified leaves. The rational explanation of such modified parts is, as the name implies, that the ancestors of the plant in question were possessed of flat pinnae, like some of their living descendants, while other descendants have become modified into tendril-bearing species. In the same way we find the spines of Cacti represent modified leaves. Other plants furnish examples of leaves being modified into floating organs, into traps for the capture and digestion of animals, into organs for the accumulation of humus, etc. Leaves are not the only organs which are subject to these modifications but each and every part furnishes similar examples. In fact all the distinguishing characters between allied species are modifications of ancestral parts and all emphasise the reality of the origin of species by descent.

(c) The argument from the existence of homologues is in reality an extension of that based on modified parts. If each species were the result of a special creative act there would be no reason why there should be found in widely divergent species parts homologous to one another. But if you regard the existing species, no 
matter how divergent in form, as derived from a common ancestor or stock, then the homologues find their natural explanation as being modifications of the same part or structure of the ancestral stock. Thus the numerous and close homologues in Funaria hygrometrica and Marchantia polymorpha are strong evidence that these two species, though now very different from one another, are descended from a common stock. Where the homology is not so striking but is still easily recognisable, as for example between Marchantia polymorpha and Pinus silvestris, we must still conclude that the two have had a common ancestry, but evidently the much greater difference shows that the divergence of the two lines of descent must be attributed to the still more remote past. The existence of homologous parts then shows the genetic relationship of classes and divisions of species much in the same way as the existence of modified parts establishes that relationship between comparatively closely similar species.

(d) The argument from Vestigial structures. Many instances are known of parts and structures of organisms which have no assignable function, and, in some cases, may be even disadvantageous to the individual. As an example of these we may cite the reduced or fugitive vegetative cells of the pollen-grain of the Seed-plants, the antipodal cells of the female gametophyte of the Angiosperms, the fruit of the banana which never produces good seed, the ligule of Selaginella and the classical example from the animal kingdomthe appendix of Man. On any theory of special creation it is incredible that organisms would be endowed with these useless, or even redundant parts, while if we regard existing plants and animals as modifications and transformations of pre-existing forms then it is natural that in these transformations the functions of some parts will be usurped or entirely superseded by others. But the parts themselves, still inherited from the ancestors in which they were functional, are now functionless and reduced, being encroached upon by new developments. They are aptly compared to the footsteps of a receding past gradually undergoing obliteration in the changing structure of the species.

(2) Evidence from Distribution.

The theory of Evolution furnishes a key for the explanation of the most various morphological facts, and the more diverse the facts it fits in with, the more convincing does the theory become. When the range of facts explained by the theory is as large as has been indicated the conviction of the truth of the theory grows almost'to a certainty. At the same time more direct proof would be acceptable. If we could isolate a number of species and watch 
their succeeding generations, recording any changes which occurred or noting their stability or immutability, we might then obtain direct proof of transformation or support of the immutability of the species observed. Such isolation experiments have been made by many observers, but the results have not been decisive. In the first case the time that has been possible to allot to them is probably vanishingly small compared to the time occupied in the transformations of species in nature : then again, the changes which have been noted have been either not transmitted or where they have been inherited there has been the doubt that they are not of sufficient magnitude to justify our regarding the new forms as distinct species.

Fortunately this experiment which is rendered unsatisfactory by the time limit of human observation and indecisive owing to the nature of the changes actually observed, has been carried out many times over in nature. The most striking records of it are found in the oceanic islands. These are islands separated from the continents by large tracts of sea. They have originated, in more or less remote periods of the past, from the outbursts of submarine volcanoes, or from the upheaval of parts of the ocean floor. The present population of plants and animals of these islands always resembles the inhabitants of those continents from which they are most accessible more closely than those of more distant lands. Hence it is known that it is derived from chance colonists, borne on the air, it may be as seeds or spores, on the sea, or even by birds. In cases where isolation is very complete the periods of time elapsing between such colonisations may be very great. Hence the species inhabiting these islands, derived from the continent from which the islands are most accessible, develop there but slightly influenced by crossing with the parent stocks from which they have been derived. If species were immutable, the species now found on these islands should be, so far as climatic conditions permitted, the same as those on the colonising continent. On the other hand, if species are mutable we would expect to find a larger or smaller number of species on these islands not found on the continent, these new species having arisen by transformation of some of the colonising species. The proportion of new species would in this case depend on the completeness of the isolation, the rate of transformation and the period of isolation. We may illustrate the general relationship found in these cases by a few facts drawn from Darwin's account of the flora or plant population of the Galapagos Islands.

This group of islands lies on the equator due west of Ecuador, about 500-600 miles from the coast of South America. They are 
volcanic in origin, and the fact that some of their craters are still active shows that the islands have been formed in comparatively recent geological time. When Darwin first landed on the archipelago he was disappointed with the uninteresting appearance of the stunted vegetation. He noticed, however, that this dwarfed vegetation showed an unmistakable relationship with the flora of South America, being composed of the same orders and classes. On further examination he was astonished to find that most of the species were different from those found in South America. Thus of the 175 species of flowering plants found by him in the Galapagos, roo are not known elsewhere in the world, although some of them belong to South American genera. Of the twenty-one species of the order Compositæ found on the Galapagos twenty are found only there. They belong to twelve different genera, of which ten are peculiar to the archipelago. His examination brought to light another very interesting point. Where a genus was widely spread over the archipelago, the species of that genus found in the different islands differ from one another. Thus a genus of a tree-like composite, Scalesia, which is spread over six of these islands and is found nowhere else in the world, is represented in each island by a different species. Similar instances of this kind of distribution of other genera of plants were found. The analysis of the animal population gave results quite like those of the vegetation, and it is not surprising to learn that it was these observations made on the Galapagos Islands which were directly responsible for turning Darwin's thoughts to the problem of the origin of species. The inevitable conclusion which has to be drawn from these records is that the descendents of most of the colonists since their transit from South America have become so transformed that they no longer belong to the same species as their relatives remaining in South America. In the case of the genus Scalesia and many others this transformation has been so profound that the descendants of the colonists and those of their stay-athome relatives can no longer be classed as belonging to the same genus. The occurrence of different species on the six different islands affords conclusive evidence that new species have arisen in the archipelago, and that the observations cannot be explained by the dying out of the parent stock on the adjacent continent. The large number of species peculiar to the islands, and the fact that these species belong to the most various classes and orders, also preclude this view. The smaller number of species common to the islands and the adjacent continent may be attributed to the stability of these species, but with greater probability to their more recent 
introduction. The fact that the islands have been of comparatively recent formation shows that the transformations of species occur within limits of time which make these changes of prime importance in the evolution of organisms in the history of life upon the earth.

(3) Evidence from the Geological Record.

Decisive evidence on the mutability species would be afforded if we possessed complete collections of the flora or fauna of some district extending back over a sufficiently long period. In such collections, herbaria of dried plants for example, if species were immutable we would find the plants between the earliest pages exactly corresponding in their specific characters with the most recently collected specimens, while if they were mutable the recent specimens would be found to diverge from those between the earlier sheets in proportion to the time elapsing between the two periods of collection and to the mutability of the species considered.

While scientific collections do not extend over nearly great enough periods to show great transformations, and are not by any means sufficiently complete to establish small changes, the sedimentary rocks of the earth's surface do form such chronological collections which extend into sufficiently remote periods to record the great transformations. Unfortunately the record is so incomplete that the minor and more gradual changes are but seldom registered in what is preserved. The strata of these rocks-the pages of the majestic volumes of the Book of Time-were laid down on the margins of past continents and in great inland seas. They contain the remains of past floras and faunas, which lived and died on the surface of sediments accumulated before them, or were carried short distances to be laid between the leaves of nature's record. Here, in fortunate instances, they were preserved by the infiltration of mineral solutions and now form fossils giving us some indication of the remote ancestors of 'our present living beings. The study of these fossils clearly establishes that the further we turn back the pages of the geological record the more widely does the flora preserved there differ from that which is now around us. In recent rocks we find the remains of existing orders and classes. More seldom have existing genera and species been found. In earlier sediments no records of flowering plants are seen, while ferns and even ferns producing seeds are plentifully represented. In the earliest rocks of all not only flowering plants but even ferns leave no record, and the only specimens found are such as belong to the more simple and primitive groups. A parallel record of animal remains, more complete seeing that animal structures lend them- 
selves more readily for preservation, trace the animal kingdom from its simple beginnings to its present marvellous complexity. Thus in this great book we find written in bold outline the history of organic evolution. While in most instances the record is only in outline some details of fascinating interest have been preserved. Thus botanists have been able to trace the origin of seed plants from fern-like ancestors, and their investigations have made us acquainted with the form and the minute structure of many seedbearing ferns, which are genetic links between the present seed plants and primitive ferns. Very recently fossils have been found probably illustrating similar connecting-links between the mosses and ferns. Similar discoveries of much more complete connecting series in the animal kingdom were made in the last century, so that to-day the geological record stands as one of the most convincing proofs of the theory of evolution.

While naturalists are pretty well agreed that the vast numbers of species, as we see them on the earth at the present day, have arisen by descent from pre-existing less numerous and more simple forms, there is no such general agreement as to the manner in which this evolution and these transformations of species have come about.

Darwin's great contribution towards the solution of this problem was the theory of natural selection. And it is to this theory that the name of Darwinism is properly applied.

Starting from the observations that the individuals of the progeny of any two parents, or of any single bisexual individual, differ, or vary, among themselves, and that, as many variations are heritable, human selection can produce special races from a common stock, Darwin conceived that if some natural cause acting in a similar manner to human selection could be shown, then an explanation would be given as to how a certain line of descendants of a species is caused to diverge more and more from its average character until a new species is formed. Reflecting that many more individuals are born in each species than can come to maturity, and that in the struggle for existence many perish, while only the fittest - or those endowed with variations which enable them to 'combat better external and internal conditions-survive, Darwin recognised in this weeding-out process a natural factor comparable to human selection in the artificial production of various breeds and races. This factor he called Natural Selection.

None can deny that natural selection operates to eliminate the unfit, but biologists are not agreed as to how far the observed 
variations are adequate to account for the transformations of species.

The older attempt, associated with the name of Lamarck, to explain the transformation of species, assumed that, as in the individual, the use of a part secures its perfection and development, while if it falls into disuse, atrophy and reduction overtakes it, so in the race those parts which were used and exercised are developed while those which are superfluous and disused disappear and atrophy. The characters produced by exercise are evidently characters acquired by the individual, and in proportion as experimental evidence has accumulated showing that such characters are rarely if ever transmitted, Lamarck's theory of the transformation of species has lost ground.

Another possible cause contributing to the transformation of species is the segregation of determinants in the formation of the germ cells, and the new combinations of characters resulting from fertilisation revealed by research following Mendel's discoveries. But here again, although certainly new races and varieties are formed by this process, all naturalists are not convinced that the characters produced, or made apparent, in this way are of sufficient importance to transgress the limits of species and genera.

Thus while the theory of evolution, or the origin of species by descent, stands established by the consistent evidence of many lines of research, the method by which evolution acts remains a mystery. Towards the solution of this mystery we possess guiding suggestions, it is true; but whether they will prove adequate, or will have to give place to others when knowledge is extended, time alone can show. 


\section{INDEX}

ACHROMATIC fibres, 252, 253.

- spindle, 252, 255 .

Acquired characters, $260,278$.

Air. Dissolved air does not destroy cohesion, 226.

Air-chambers in Marchantia, I23, 124. Air-passages in apophysis of Funaria, 142.

Elodea, 42.

leaf of Aspidium, 155.

- - leaf of Aspidium,

- - - Ranunculus, 222.

- - - Selaginella, 172.

- - root of Scilla, 24I, 242.

- - stem of Pinus, I84.

- - - Ranunculus, 217, 222.

- - - Selaginella, I72.

Alcohol, coagulation by, 29 .

- production of, 25,3 .

Allelomorphs, 26I, 262, 264, 265.

- may influence each other, 268.

Alternation of generations, 120.

- in Aspidium, I67.

- - Funaria, 142 (Fig. 31).

- Marchantia, I33 (Fig. 30).

- Pinus, 209, 212 (Fig. 72).

- - Polysiphonia, I20.

- - Ranunculus, 236 et seqq.

- Scilla, 248.

Amino-acids, 16, 17, 28.

Anabolism, $5 \mathrm{I}$.

Analogues, I68.

Andalusian fowl, 265.

Angiosperms, 21 5, 237.

- subdivision of, $239,240$.

Angularity, 26I (Fig. 92).

Animal kingdom, alternation in, 120.

- - energy supply for, $47,5 \mathbf{I}, 67$.

- - food of, 66, 102.

- - nitrogen supply for, 66,67 .

Annulus of Aspidium, I62.

- - Funaria, I4r.

Anther, 23r, 246.

Anther-lobe, 23r.

Antheridiophore, 128.

Antipodal cells, 234, 273.

Apophysis, 140.
Appendix of Man, 273.

Aquatic plants, I2I, I67.

Archegoniates, 168, 213.

Archegoniophore, 130.

Archesporium, 231.

Ascent of sap, cohesion theory of the, I 56, 244 et seqq.

Ascocarp, roo.

Ascospore, roo.

Ascus, roo.

Asexual reproduction of Chlamydomonas, 39.

- - - Funaria, 136, 143, 144.

- - Marchantia, I25, 126.

- - Mucor, 94.

- - Penicillium, 99, roo.

- - Polysiphonia, r16.

- - Vaucheria, 87, 89.

- - Volvox, 78.

- - yeast, 25, 26.

Aspidium flix-mas. Lectures XIX. and XX., 146-169 (Figs. 32, 33, $34,35,36,37,3^{8}, 39,40,4 \mathrm{r}$, 42,43 ).

- - amphibious, r67.

- - annulus of, I62, I63.

- - antheridia of, I64 (Fig. 42 ).

- - apical cell of, $x_{52}$.

- - archegonia of, $\mathbf{1 6} 5$.

- - bast of, 149, 150 .

- - conducting tracts of, I48, I49, I 5O, I 5 I, I 53, I 54 .

- endodermis of, 151 .

- - epidermis of, I $_{54}$ (Figs. 37 and 38).

- - fertilisation of, $\mathbf{I} 66$

- - fundamental tissue of, r48, 149 .

- - gametophyte of, I64.

- - germination of spores of, I64.

- - guard-cells of, I54.

- - holophytic, I55.

- - indusium of, 161.

- - leaf-stalk of, r53.

- - leaves of, 153-159.

- - - origin of, 147 .

- life-history of, $166,167$.

- lip-cells (stomium) of, 162. 
Aspidium filix-mas, mesophyll of, 154 , I55.

- ova of, 165.

- palisade parenchyma of, I55.

- - pericycle of, $\mathbf{I} 5 \mathbf{I}$.

- photosynthesis of, 157.

- - pinnæ of, 154 .

- - rachis of, $\mathbf{I}_{53}$.

- respiration of, $\mathbf{I} 57$.

- - rise of water in, 155 .

- root of, 152, $_{553}$.

- root-cap of, $\mathbf{r} 53$.

- - sclerenchyma of, 152.

- - sieve-plates of, 150 .

- sieve-tubes of, 150 .

- sori of, I6r.

- sperms of, 165 .

- spongy parenchyma of, $\mathbf{I} 55$.

- - sporangia of, 161,163 .

- - spores of, 163 .

- - stem of, $146-151$.

- - stomata of, $\mathbf{1}_{54}$ (Figs. 37 and $3^{8}$ ).

- transpiration in, 157.

- - tracheids of, 149.

- wood of, 149 .

Assimilation, 26, 31, 32.

Autoclave, 59.

Auxiliary cell, 1 I8 (Fig. 2I).

BAcillus coli, 63 .

- pyocyaneus, 63 .

- subtilis, 63 .

- vulgaris, 62.

Bacteria. Lectures VII., VIII., IX., 52-67 (Fig. ro).

- aerobic and anaerobic, 63 .

- analysis of, 57 .

- beer and wine, concerned in souring, 65.

- cell of, 85 .

- cell-structure of, 54 .

- cell-wall of, 55 .

- chemosynthesis of, 64 .

- cilia of, 53 .

- colonies of, $6 r$.

- culture medium for, $5^{8}$.

- holozoic, 62.

- isolation of, 60 .

- lactic acid, 65.

- metabolism of, 57 et segq.

- motion of, 52, 62 .

- nitrifying, 63 .

- nitrogen-assimilating, 63 .

- nitroso- and nitro-, 64 .

- nucleus of, 54 .

- parasitic, 62 .

- pathogenic, 65 .

- physiological species of, 65 .

- pure culture of, 60 .

- putrefactive, 62 .
Bacteria, rate of reproduction of, 55 .

- reproduction of, 55 .

- respiration of, 63 .

- saprophytic, 62,63.

- size of, 53 .

- smear-preparation of, 56 .

- source of, 52 .

- spontaneous generation of, $5^{8}$.

- spores of, 56 .

- sub-cultures of, 62 .

- sulphur, 64 .

- various forms of, 53 .

- views as to nucleus of, 54 .

Banana fruit, 27.3.

Bark of Pinus, 198, 199.

Bast (= phloem), r49, I50, I 55, 173, 186, 219.

- of Pinus, 182, 186, 187, 198.

- Ranunculus, 217, 219, 220, 222.

- primary, 186, 219, 220.

- secondary, 186.

- transport of materials in, 157,198 .

Bast-parenchyma, I $_{5} \boldsymbol{x}$.

Beetroot, cells of, 17 .

Bordered pits, 193, I94, I95, 197, 227.

Bract-scale of Pinus, 205.

Brownian motion, 24, 25.

Bulb of Ranunculus, 215.

- - Scilla, 240, 241 .

Bundle. See Conducting tract.

CACTI, 272.

Calibration of ghost-micrometer, 33, 34 .

Calorie, 32,

Calyptra, 140.

Calyx, 230, 245.

Cambium, 183, 186, 192.

- interfasicular, $\mathbf{1} 86$.

Carbohydrates, 10, 16, I7.

- energy of, $32,46,50$.

Carbon, source of, for green plants, 43,49 .

- sources of, for holozoic plants, $3 \mathbf{I}, 49$,

Carotin, 46.

Carpels of Pinus, 205.

- - Ranunculus, 232, 236.

- Scilla, 245, 249, 246.

Carpogonium, 117.

Carpospore, IIg.

Casease, ror.

Catabolism, $5 \mathbf{I}$.

Catalase, ror.

Catalysts, 28, 236.

Cell, definition of, 84 .

- of epidermis of Tradescantia virginiana, 3 (Fig. 2).

Cell-plate, 253.

Cell-wall of bacteria, 55 .

- - cell of beetroot, I $4 .^{4}$

- Chlamydomonas, 36 .

- cell of Elodea canadensis, 42. 
Cell-wall of cell of Rhododendron petal, Chromatin, hereditary substance or I4, I5.

- - - Tradescantia virginiana, 3 .

- - yeast, 23, 24, 25, 26, 28, 31.

- chemical characteristics of, I0, 55 .

- permeability of, ro.

- physical characteristics of, 9 .

- tenacity of, 10, 19, 25.

Cellulose, ro, 22, 36 .

Characters, acquired, 260

- differentiating, 260.

- dominant, 262.

- inherited, 260.

- recessive, 262 .

Chemotaxis (= chemotropism), 93 .

- of Mucor, 93.

- - sperms, 130, 139, 166.

Chlamydomonas. Lectures V.-VI., 35-48 (Fig. 7).

- asexual reproduction of, 39 .

- carbon, source of, for, 49.

- cell-wall of, 36,39 .

- chlorophyll of, 37 .

- chloroplast of, 37 .

- chromatophore of, 37 .

- cilia of, 36,39 .

- contractile vacuoles of, $37,5 x$.

- food of, 4 I.

- gametes of, 39 .

- holophytic, 49.

- irritability of, $3^{8}$.

- motion of, $35,3^{8}$.

- nitrogen for, source of, 49 .

- nucleus of, 37,39 .

- oxygen, evolution of, by, 41 .

- photosynthesis in, 41-47.

- protoplasm of, 35 .

- pyrenoid of, 37.

- response in, $3^{8}$.

- sexual reproduction of, 39 .

- starch in, 37, 4r, 50 .

- stigma of, 37 .

- spontaneity of, 38 .

- source of carbon for, $4 \mathrm{I}$.

Chlorophyll in colloidal state, 46 .

- cont ains magnesium, 46.

- properties of, $45,46,47$.

- $a$ and $b, 46$.

- solution of, 45 .

- purveys solar energy to living beings, $5 x$.

Chloroplast, function of, 46,47 .

- of Chlamydomonas, 37 .

- Elodea canadensis, 42, 43.

- of Fucus, ro5.

- Selaginella, r72.

- Spirogyra, 68.

- - Vaucheria, 33.

- Volvox, 76.

Chromatin, 25r, 253. germ-plasm, III, 254 .

- equal amounts in sperm and ovum, III, 254 .

Chromatophore, 37, 231.

Chromomeres, 25I, 256, 257.

- division of, $252,254,255,256$.

Chromosomes, 251, 253, 254, 255.

- cleavage of, 252, 254, 255 .

Circulation of nitrogen, $65,66,57$.

Classification, evidence on evolution, 271.

Coagulation, r6, r8, 22, 29, 3r.

Conocyte, 85, 95, 97, 237, 238, 248.

Co-enzyme, 30,73 .

Cohesion-Theory of the ascent of water, I 56,224 et seqq.

Collenchyma, 243 .

Colloidal state, 19, 20.

- - enzymes in, 28, 29.

Colloids, r9, 20.

- particles of, 20, $2 \mathrm{r}$.

- past history of, 22.

- produce very little osmotic pressure,

- surface of, 20, 2 r.

Colonies of bacteria, $6 \mathbf{r}$.

Columella of Mucor, 94.

- Funaria, r4r.

Combustion of carbohydrates, 50 .

- heat of, 32,50 .

Companion-cells, 220.

Conceptacles of Fucus, 107.

Conducting tracts (= vascular bundles).

- - development of, in Pinus, 186.

- - of Aspidium, I47, 148, 149, 150,

- - "closed," 218.

- - in embryo, 250.

- . of Pinus, 182,183 .

- - Ranunculus, 217 et seqq.

- - root of, in Scilla, 24r.

- - Selaginella, 173.

- - subdivision of, 227.

- " "open," 218.

- paths of, in stem of Aspidium, 148.

- - - in stem of Selaginella, 173 .

- - - - - Pinus, r82 et seqq.

- - - - Ranunculus, $2 \mathrm{r} 7$ et seqq.

- - - - - Scilla, 243, 244.

_ _ Dicotyledonś and Monocotyledons, 244 .

- - - leaves of, Dicotyledons and Monocotyledons, 245.

Cone of Pinus, (see seed-cone and pollen-cone), r99, 204 .

- Selaginella, 173, 2 r3.

- - - and of Pinus compared, 213.

Congo Red-a colloid, r9.

Connective, 23 r.

Contractile vacuoles, eliminate waste products, $5 \mathrm{I}$. 
Contractile vacuoles, mechanism of, $5 \mathrm{I}$, 52.

- where found, 52 .

Cork-cambium, 198.

Corolla, 230, 245 .

Cortex, function of, 199.

Cotton-wool, fibres of, ro.

- use of, in bacteriology, 59.

Cotyledons, 176, 209, 239, 240.

— of Pinus, 209.

- Ranunculus, 235.

- Scilla, 239, 240.

- Selaginella, r76.

Cover-glass, $\mathbf{x}$.

- cleaning of, 2,3 .

Cross, 262.

Cross-pollinate, 262.

Cross-pollination. Reciprocal, 262.

Crystalloids, 19, 20.

- solution of, $\mathbf{x}$, 12 .

Cystocarp, Irg.

Cytase, ror.

DARWIN, 270, 274, 277.

Darwinism, 277.

Death and reproduction, 126.

Decay, 52, 125.

Dehiscence of pollen-sacs, 200, 232, 246.

Delafield's hrematoxylin, a stain for Mucor, 96.

Descent, theory of, Lecture XXX., 270278.

Determinants (factors), 263.

- segregation of, 264,278 .

- uninfluenced by each other, 265.

Dextrose, a product of inversion, $: 0,34$.

Diastase, 50, Ior.

Diaster, 253.

Dicotyledons, 240, 244, 245.

Diffusion of crystalloids, 11, 12, 19, 20.

Dicecious. See Unisexual.

Diploid, 257.

Dispersion of light by colloids, 20.

Dispireme, 253.

Dissemination of spores, 100, 132, I4I, $163,200,232,246$.

- - seeds, 209, 248.

Distribution and evolution, 273.

Disuse and atrophy, 277.

Division of labour, $85,89,107$.

Dome of bordered pit, 193 .

Dominance, 262.

- complete, 264.

- incomplete, 264 .

Dominant character, 262.

- double, 266.

Double dominant, 266.

- recessive, 266.
ELATERS of Marchantia, I31.

Elodea canadensis, cell of leaf 43 (Fig. 9).

- - chloroplasts of, $42,43,47$.

- - cytoplasm of, 43,48 .

- - evolution of oxygen by, $4 \mathrm{I}$ (Fig. 8).

- nucleolus of, 42,47 .

- nucleus of, 42,47 .

- photosynthesis in, $4 \mathrm{r} \cdot 47$.

- streaming of protoplasm of, 48 .

- - vacuole of, 43,47 .

Embryo, differentiation of, 250.

Embryo-sac (= Megaspore), 206.

- of Pinus, 206, 2 Ir.

- Ranunculus, 234 .

- - Scilla, 246.

Emulsin in Penicillium, ror.

Emulsion, 26.

Endarch, 187.

Endodermis, 151, 155, 173, 182, 223, 242.

Endosperm ( = Female gametophyte), 206.

- of Pinus, 206, 207, 209, 210, 238, 248.

Endotryptase, 30 .

Energy, source of, for plants, 50.

- supplied by combustion, $3 \mathbf{1}$.

- ultimately solar, 5 r.

Enzymes, 28, 29. 30, 31, 33, 34 .

Epidermis of leaf of Tradescantia vir. giniana, 4 (Fig. 2), 17.

Equatorial plate, 25I, 253.

Evaporation, protection from, 124, 125.

Evolution. See Theory of Descent.

$F_{1}, F_{2}$ and $F_{3}$ generations, 262 .

Factors (determinants), 263.

Fermentation, 33 .

Fibrous, layer of pollen-sac of -

- Pinus, 200.

- Ranunculus, 232.

- Scilla, 246.

Filament, 23 I.

Flowers, 230, 240, 245.

Foot, 140, 166, 176.

Fossil seed-plants, $21 x, 277$.

Fossils, I7I, 21 1, 276.

Fritillaria imperialis, seeds of, for $\mathrm{Mi}$ tosis, $25^{8}$.

Fruit, 236.

- syncarpous, 248.

Fucoxanthin, ro5.

Fucus platycarpus. Lecture XIV., I04II2 (Fig. 19).

- - antheridia of, 108.

- chromatophores of, 105.

- conceptacles of, ro7.

- - cortex of, ro4, ro5.

- - division of, labour in, 107.

- - gametes of, ro8.

- - growth of, I06.

- - hairs of, ro4, 106. 
Fucus platycarpus, medulla of, ro6.

$\rightarrow$ mid-rib of, 104, 106, 107.

- oogonia of, 108.

- ova of, ro8.

- - photosynthesis of, ro5.

- - sexual reproduction of, I07-112.

- - sperms of, rog.

- - transport of materials in, 106, 107.

Funaria hygrometrica. Lecture XVIJI., I35-I 45 (Figs. 26, 27, 28, 29, 30, 3I).

- - annulus of, 141. -

- - antheridia of, 137 .

- - apex of stem, 136, 137 .

- - apophysis of, I40, I42.

- - archegonia of, 138 .

- - asexual reproduction of, 136,143 ,

- calyptra of, $\mathbf{1} 40$.

- columella of, 140 .

- cortex of, 136 .

- - embryo of, 139 .

- - germination of spores of, 136 .

- - leaves of, 136,137 .

- operculum of, 140 .

- - ovum of, 138 .

- - paraphyses of, r 37 .

- - peristomium of, I $_{4} \mathrm{I}$.

- - protonema of, 136 .

- - rhizoids of, 136 .

- - seta of, 140 .

- - sperms of, 138 .

- stem of, $\mathbf{1}_{3} 6$.

- - stomata of, I40, 142.

- theca of, $\mathbf{x} 40$.

- - vagina of, $\mathbf{r} 40$.

Fundamental tissue of Aspidium, r48, I 49.

- - Pinus, 183 .

- - Ranunculus, 217.

Fungi, 97 . Scilla, 244.

- nutrition of, I02.

Fusion-nucleus, 234, 248.

GALAPAgos Islands, 274.

Gametangia of thallophytes and archegoniates, $1_{33}, r_{34}$.

Gametes, 39, 40.

- differentiation of, Iro.

- haploid, 257.

- of Aspidium, 165.

- Chlamydomonas, 39.

- Fucus, 108, 109.

- Funaria, 138.

- Marchantia, 129, 130.

- Mucor, 94, 95.

- Penicillium, roo.

- Pinus, 207, 208.

- Polysiphonia, rI7.

- Ranunculus, 234, 235.
Gametes of Scilla, 247.

- Selaginella, I75, 176.

- Spirogyra, 70, 7I, 73 .

- - Vaucheria, 87, 88.

- Volvox, 79, 80.

- pure, i.e. do not carry the two determinants of a pair of allelomorphs, 264 .

Gametophyte, 142.

- aquatic, $133,164,178$.

- cœenocytic, 237, 238, 247.

- haploid, 257.

- male and female, 142, 177 .

- - - of Pinus, 207, 208, 210.

- - - - Ranunculus, 234, 235, 237.

- - - - Scilla, 249.

- parasitic, 208, 2ro, 2 II.

- of Aspidium, 164, 165, r66.

- Funaria, 142.

- Marchantia, I42.

- Polysiphonia (=carposporic plant),

- Selaginella, 175, 177 . [II9.

Gaseous interchange, $124,140,142,1_{55}$, I72, I8I, I84, 217, 222, 24I, 242.

Gel, 22.

Gelatine, example of co.loid, 20, 26.

- in colloidal state, 20.

- to damp ciliary motion, 40 .

- - solidify culture medium, 33,59 .

Genealogical tree, 272.

Gentian violet, stain for volvox, 82 .

- - - - bacteria, 56 .

Geological record and evolution, 276.

Geotropism of Mucor, 93, 94.

Germ cells, 8r.

Germ-plasm in nucleus, $\mathrm{IIx}$.

- the chromatin, III, 254.

- not influenced by surroundings, 26r.

Ghost-micrometer, 34 .

Glucose formed from starch, 50 .

Gravitational stimulus, 94, 99, 126, 236.

Green plants build organic from inorganic materials, 49 .

- supply food and energy to rest of living nature, $47,49,51$.

Guard-cells, I43, I54, 181, $216,243$.

Gums, 22.

Gymnosperms, 215, 237.

HAPLOID, 257.

Heat, discontinuous, sterilisation by, 59 .

- of combustion, 32, 50.

Hereditary material. See Germ-plasm.

Heredity, Lecture XXIX., 260-269

(Figs. 92, 93, 94).

Heterosporous plants, $178,211,213$.

Heterospory in Pinus, 209, 211.

- Ranunculus, 237. 
Heterospory in Selaginella, 178.

Heterozygote, 264.

Heterozygous, 264 .

Holophytic nutrition, 49 .

Holozoic nutrition, 49, 102.

Homologues, $168,213,272,273$.

Homozygotes, 266.

Hook, Robert, 84.

Hunger stimulus and sexual reproduction, 40.

Hydrolysis, x6, 28.

Hygroscopic curvatures of elaters, $\mathbf{r}_{32}$.

- - peristomium, r4I.

- - seta, $x_{4} \mathrm{x}$.

IMMORTALITY of Chlamydomonas, 72.

- - germ cells, 8r.

- - Spirogyra, 72.

- - unicellular organisms, 82.

Indusium, r6r.

Insect-pollination, 234, 237, 247.

Integument, 206.

- of Pinus, 206.

- Ranunculus, 234.

- Scilla, 248.

Internal supports of tracheids and vessels, 228.

Invert sugar, 30,34 .

Invertase, 30,34 .

Iodi, liquor, 8.

Isodiametrical cells, 105.

Isolation experiments. 274.

Karyórinesis. See Mitosis.

Koch, $5^{8 .}$

LACTIC acid, 65.

Lactose, 65 .

Lamarck, 278.

Land-flora, 12 r.

Leaf-pigment, 46 .

Leguminous plants, nodules of, 67 .

- - tendrils of, 272.

Levulose, a product of inversion, 30,34 .

Life, 33 .

Light-stimulus and gemmæ, 126.

Ligule, I7 r, 205, 273.

Linin, 25r, 253 .

Lipase in Penicillium, ror.

Lip-cells (stomium), 162.

Lipoids, 16, 17.

Liquor iodi, 8.

Lock and key theory of enzyme action, ro2.

Loculus of ovary, 246.

MAGNESIUM, essential element in protophlasm, 16.

- in chlorophyll, 46 .

Maltase, 50.
Marchantia polymorpha. Lectures XVI., XVII., x21-r34 (Figs. $22,23,24,25$ ).

- - air-chambers of, 122, 124.

- - alternation of generations in, 133 (Fig. 30).

- - antheridia of, 128.

- - antheridiophore of, 128.

- - archegoniophore of, 130.

- - archegonium of, $x 30$.

- - asexual reproduction of, $125,126$.

- cellular differentiation of, 125 .

- cupules of, 126.

- - death and reproduction of, 126 .

- - dehiscence of sporangium, 132.

- - elaters of, r3r.

- embryo of, $13 \mathrm{r}$.

- - fertilisation of, $\mathrm{x} 30$.

- foot of, rizr.

- - gemmæ of, r26.

- mid-rib, 122.

- oosperm of, $x_{3}$ x.

- ova of, rзo.

- - photosynthetic cells of, 124 .

- - pits in cells of, 124 .

- - rhizoids of, 122, 124 .

- - scales of, 122.

- sperm-cells of, 129.

- sporangium or spore-capsule of, r3I.

- - spores of, $13 \mathbf{r}$.

- stalk of, $13^{2}$.

- - thallus of, 122 .

- ventilating pores, 123.

Measurement, microscopic, 23.

Medullary rays, $186, x 95, x 97$.

- - secondary, $x 86$.

Megaspores, 173 .

- of Pinus, 206, 207, $21 \mathrm{x}$.

- - Ranunculus, 234, 23.5 .

- Scilla, 248, 249.

- Selaginella, $173, x 75$.

Membrane, semi-permeable, $x_{4}$.

Memory, physiological, 39.

Mendel, 26r, 278.

Mesarch, 187.

Mesophyll cells, supported by osmotic pressure, 155 .

- laboratories for synthesis, 157.

- of Aspidium, 155 .

- Pinus, 18r.

- Ranunculus, 222.

- Scilla, 245.

Metabolism, 57,64 .

Microscope, measurement with, 23.

Microscope, parts of the, $I, 2$.

- use of, $3,4,5,6,7$.

Micro-organisms, Lectures VII., VIII., and IX., 52-67. See Bacteria.

Micropyle, 206. 
Micropyle of Pinus, 206.

- Kanunculus, 234.

Microspores, 173, 201, 212, 213, 232, $237,249$.

Milk, souring of, 65 .

Mitosis, Lecture XXVIII., 250-259 (Figs. 90, 9r).

Modified parts and evolution, 272.

Monaster, 253.

Monocotyledons, 240, 244, 245 .

Morphology, evidence from, $27 \mathrm{I}$.

Mortality of organisms, 82 .

Mould, blue. See Penícillium glaucum, Lecture XIII.

- white. See Mucor, Lecture XII.

Mucor, Lecture XII., 91-96 (Fig. 17).

- a parasite, 95 .

- a saprophyte, 95 .

- anaerobic modification of, 96.

- asexual reproduction of, 94 .

- chemotropism of, 92,93 .

- cœnocyte of, 91,95 .

- cœenocytic gametes of, 95 .

- columella of, 94.

- curvature of, 93 .

- cytoplasm of, $91,92$.

- nycelium of, $9 \mathrm{r}$.

- nuclei of, 92, 94.

- nutrition of, 95 .

- respiration of, 96 .

- response to gravity of, 93 .

- - - light of, 93 .

- sexual reproduction of, 94,95 .

- sporangiophore of, 94 .

- sporangium of, 94.

- spores of, 94.

- zygospore of, 95 .

Multicellular plants, 23, 76, 82 .

Mutability of species, $274,276$.

NATURAL selection, 277 .

Nitrates, 4I, 49, 63, 64, 66, 67, 69, 78, 85,96, 107, I $16,142,156$.

Nitrifying bacteria, 63,64 .

Nitrogen, free, utilised by bacteria, 63 .

- circulation of, $65,66,67$.

- source of, for green plants, $49,66$.

- sources of, for holozoic plants, 3 r, 49,

Nucellus, 206.

- of Pinus, 206, 209.

- - Ranunculus, 234.

- Scilla, 246.

Nuclear fluid, 250, $25 \mathrm{I}$.

- division. See Mitosis.

- membrane, $250,256$.

- net-work, 250.

- plate, 253.

- thread, 25r, 255.

Nucleinic acid, 251.

Nucleolus, 25x, 253, 255.
Nucleus contains the hereditary material (germ-plasm), IIr.

- does not arise de novo, 250.

- granular in appearance, 25I.

- of cell of epidermis of leaf of Tradescantia virginiana, $3,4,6$.

- resting, 250.

Nutrition, holophytic and holozoic contrasted, $49,62$.

OCEANIC Islands, 274.

Oosperm, 89.

Operculum, 140.

$[89, \mathbf{1 2 0}$.

Optical section, $6,17,23,36,47,74,83$,

Osmotic pressure, II, I2, 13, 14, 19.

- - a compression member, 93, 99, I 59 .

- - distends cells, I55, I 56, 228.

- - of vacuole, 14,93 .

Ovary, $233,237,246$.

Ovules of Pinus, 205.

- - Ranunculus, 233.

- - Scilla, 246.

Oxygen, evolution of, $4 \mathrm{I}$.

PALISADE parenchyma of Aspidium, 155. - of Ranunculus, 222.

Paraphyses, I37.

Parasitism of bacteria, 62 .

- - gametophyte, 177, 208, 210.

- - Mucor, 95 .

- - sporophyte, I42, 143 .

Parthenogenesis, IrI.

Passage-cells, 242.

Pasteur, $5^{8}$, ror.

Pathogenic bacteria, 65 .

Pectose, 253.

Penicillium glaucum. Lecture XIII.,

- - asci, ror.

[67-103 (Fig. 18).

- - ascogenous hyphæ of, 100.

- - ascospores, Ior.

- - asexual reproduction of, 99, roo.

- - branches of, 99.

- - chemotropism of, 99.

- - cœnocytes of, 97.

- conidium of, 99.

- - enzymes of, ror.

- - gametangia of, roo.

- - geotropism of, 99.

- - hyphæ of, 97.

- - mycelium of, 97 .

- - nitrogen, source for, I02.

- - nuclei of, 97.

- - nutrition of, ror.

- - oil drops in, 97.

- - omnivorous, ror.

- - osmotic pressure in, 99.

- - respiration of, ro3.

- - rigidity of, 99 .

- - sexual reproduction, Ioo, ror. 
Penicillium glaucum, sterigmata of, 99. - - tartaric acid changed by, ror.

Peptide linkage, $\mathbf{1 7 .}$

Perianth, 246.

Pericycle, 15I, 155, 223, 241.

Peristomium, 14I.

Petals, 230, 246.

Petri-dish, 6r.

Phloem. See Bast.

Photosynthesis (carbon assimilation), 4I$47,69,78,85,107,116,124,142$, I 57 .

- action of chlorophyll in, $45,46,47$.

- - chloroplast in, $45,46,47$.

- enzymes entering into, 44 .

- formaldehyde in, 44 .

- hexoses in, 44 .

- hydrogen peroxide in, 44.

- starch formed in, 44 .

- sucrose in, 44 .

- supplies food and energy to living nature, $47,5 \mathrm{x}$.

Phototropism of Mucor, 93.

Physiological species, 65 .

Piliferous layer, 241.

Pinus silvestris. Lectures XXII., XXIII., XXIV., I79-2I 4 (Figs. $5 \mathrm{I}, 52,53,54,55,56,57,58$, $56,60,61,62,63,64,65,65$, $67,68,69,70,72)$.

- air-passages of, 184 .

- apex of stem of, 185 .

- - archegonia of, 207.

- - bark of, 198.

— - bordered pits of, 193, 194, 195.

- - bract-scale of, 205 .

- - cambium of, 186, 183, 192.

- - carpels of, 205 .

- conducting tracts of, leaves and dwarf shoots, 182,183 .

- - - - development of, 186 .

- - - - long shoots, 184 .

- cortex of, 184 .

- cotyledons of, 209.

- cuticle of, $181,183$.

- - dwarf shoots of, $179,182,183$.

- - embryo of, 209.

- - embryo-sac of, 206.

- endodermis of, 182 .

- - endosperm of, 206, 207.

- epidermis of, $\mathbf{r} 8 \mathbf{r}$.

- - fundamental tissue of, 183 .

- growth in length of, 185 .

- - - thickness, 186.

- heterospory in, 209.

- - integument (= seed-coat), 206.

- - leaves of, 179.

- - life-history of, 209.

- long shoots of, 179, 180 .

- - medullary rays of, 184 .

- megaspore-germination, 206, 207.
Pinus silvestris, mesophyll of, $18 \mathrm{r}$.

- - micropyle of, 206 .

- nucellus of, 206.

- oosperm, 208.

- - ova of, 207.

- ovules of, 205.

- pericycle of, 182 .

- - pith of, 184 .

- pollen of, 200, 201, 202.

- - pollen-cones of, I\$9.

- - pollen-sac of, 200, 201, 202.

- pollen-tube-cell of, 207.

- - pollination of, 207 .

- - primary and secondary tissues of, I86.

- - procambial tracts of, 185 .

- - protoxylem, stretching of, 192.

- - resin passages of, 184 .

- - root of, structure of, 188,189 .

- root-cap of, Igo.

- root-hairs of, 190.

- - scale-leaves, 179.

- - seeds of, 206, 209.

- - seed-cones of, 204.

- - sporophylls of, 2 I3 $_{3}$.

- - spring wood and summer wood, I87.

- stamen of, 200 .

- - stomata of, r8r.

- - strobili of, 213.

- suspensor of, 208.

- tap-roots, 181.

- - tracheids in, development of, 193.

- - transfusion-tissue of, 182 .

- water, path of, in, 190.

- - wing of seed of, 206.

- - wood, sections of, 195, 196, 197.

- zonation in wood of, 188.

Pits, 106, 124, 149, 182, 193 et seqq., 221, 227.

- facilitate passage of water, 227 .

Plasmolysis, 14, 15, 18.

Polar nuclei, 234.

Pollen, 200, 201, 202.

Pollen-cones, 199, 204.

- replace dwarf shoots, 204.

Pollen-grains (= microspores), 200, 201 .

- carried to micropyles, 207.

- of Ranunculus, 232, 235.

- of Scilla, 246.

Pollen-mother-cells, 23I.

Pollen-sac (= microsporangium), 200, 20I, 202, 232, 246.

Pollen-tube, $211,235$.

Pollination of Pinus, 207.

- Ranunculus, 234, 237.

- Scilla, 247.

Polysiphonia fastigiata. Lecture XV.,

II3-I20 (Figs. 20,2 I).
alternation of generations in, 120. 
Polysiphonia fastigiala, antheridia of, II7.

- - apex of, Ir5.

- - asexual reproduction of, Ir6.

- - auxiliary cell of, XI7, II8 (Fig. 2I).

- - branches of, Ir6.

- - carpogonium of, II7 (Fig. 2I).

- - carpospores of, II9.

- - cortex of, II3, II 4 , II 5.

- - cystocarp of, 119 .

- - epiphytic, Ir3.

- - hairs of, Ir6.

- - life-history of, II9, I20.

- - medulla of, II $3, I_{4}, I_{1} 5$.

- - ova of, II7.

- pigments in, 114.

- - procarp of, II7 (Fig. 2I).

- - protoplasmic connections in, II5.

- - respiration of, 116.

- - sexual reproduction of, II7.

- - sperms of, 117 .

- - tetraspores of, II6.

- - transport in, Ir6.

- - trichogyne of, II7 (Fig. 21).

Primula "crushed strawberry," 265.

Procambial tracts, $185,250$.

Procarp, II7 (Fig. 2I).

Protease in Penicillium, 10r.

Proteid, $\mathbf{1 7}$.

Proteins, 16, 28.

- chemical nature of, $16, \mathbf{1 7}$.

- colloidal state of, 19, 28.

- in pyrenoid, 37 .

Proteolytic enzymes, 28.

Protophloem, 186, 189, 219.

Protoplasn, appearance of, $6,7,17$.

- assimilation cf, 3 I.

- chemical nature of, 16 .

- coagulation of, 16, I8, 22.

- constituents of, 16.

- death of, 17.

- gel or sol, 22.

- in colloidal state, 22, 39.

- - plasmolysis, 14, 15 .

- nature of, IO, II, I2.

- of cell of Elodea canadensis, 42.

- Chlamydomonas, 36,37 .

- - yeast, 24.

- "physical basis of life," I7, 33.

- production of enzymes by, 33 .

- respiration a physiological characteristic of, 33 .

- semi-permeable, $\mathbf{I I}, \mathbf{I} 4$.

- streaming of, 48.

Protoplasmic connections, 77, 115.

Protoxylem, I50, I73, I86, 187, I88, I92, 220, 22I, 223, 24 I.

Putrefaction, 52.

Pyrenoid, 37, 69, 77, 8r.

- of Chlamydomonas, 37.
RACHIS of Aspidium, 153.

Radial section, 188.

Ranunculus bulbosus and $R$. repens. Lectures XXV. and XXVI., 21 5-238 (Figs. 73, 74, 75 76, 77, $78,80,81,82,83)$.

- - air-passages of, 217, 222.

- anther of, 231.

- anther-lobe of, $23 \mathrm{r}$.

- - antipodal cells of, 234 .

- - archesporia of, $23 \mathrm{I}$.

- bast of, 217, 220 .

- - bundle-sheath of, $217,218,219$.

- calyx of, 230.

- carpels of, 232, 236.

- - chromatophores of petals of, 23 I.

- conducting tracts of, 217 .

- connective of, 23 I.

- corolla of, 230.

- - cortex of, 216.

- - cotyledons of. 235 .

- - cuticle of, 216.

- - dehiscence of pollen-sacs of, 232 ,

- - embryo of, 235 .

_ - embryo-sac of, $234,236$.

- endosperm of, 238.

- - ep:dermis of, 216 .

- filament of, $23 \mathrm{I}$.

- - flowers of, 230.

- - fruit of, 236.

- - fusion-nucleus of, 234 .

- gametophyte, female of, 235.

_ - male of, 237.

- - germination of pollen-grain, 235 .

- - - - seed of, 236.

— - integuments of, 234, 236.

- leaves of, 2I5, 222.

- life-history of, 236 .

- - compared with those of Archegoniates and of Pinus, $23^{8}$.

- - medullary rays of, 217 .

- micropyle of, 234 .

- nucellus of, 234 .

- - ovary of, $233,237$.

- - ovule of, 233.

- - ovum of, 234.

- palisade parenchyma of, 222.

- petals of, 230.

- - pith of, 217 .

- polar nuclei of, 234.

- - pollen-grains of, 232, 236.

- poilen-mother-cells of, 231 .

- pollen-sacs of, 232.

- - pollination of, 234 .

- root-pressure in, 228.

- - roots of, 223.

- - seed of, $230,236$.

- - sepals of, 230.

- - spongy parenchyma of, 222.

- - sperm-cells of, 235 .

- - stamens of, 23I, 236. 
Ranunculus repens, stems of, 215 .

- - structure of stems of, 216 .

- - stigma of, 233, 234, 235, 237.

- - stomata of, 2 r6, 222.

- - style of, 233, 234, 237.

- - synergidæ of, 234 .

- - tapetum of, 23 I.

- thalamus or axis of, 230.

- - water, rise of, in, 224 et seqq.

- - wood of, 217, 220, 221 .

Recessive character, 262.

- double, 266.

Reconstruction, microscopic, 68, 74, 89.

Red sea-weeds, a source of bacteria, 52 .

- - Polysiphonia, example of, 113 .

Reduction-mitosis, 255 et segq.

- in Fucus, 257.

- Polysiphonia, 257.

- Seed-Plants, 257.

- - Spirogyra, $25^{8}$.

Reproduction, asexual, 39.

Asexual reproduction.

- sexual, 39, 72. See Sexual reproduc-

- hunger stimulus and, 40.

- of Chlamydomonas, 39.

- - Spirogyra, 72.

- origin of, 40 .

Resin ducts, $184,196,198$.

Respiration aerobic, $49,50,116,157$.

- anaerobic, 3I, 32, 33 .

- - of yeast, $3 \mathrm{I}$.

- - bacteria, 63 .

- - Mucor, 96 .

- - Penicillium, ro3.

- defined, 32.

- dependent on enzymes, 33.

- intermediate stages of, unknown, $5^{\mathrm{r}}$.

- reverse of photosynthesis, 49 .

Reversible actions, 29.

Rhizophore of Selaginella, $\mathbf{I} 7$ I.

Rhododendron petal, I5, 17, I9 (Fig. 4).

Rigidity of cell, $x 9$.

- - leaf-stalk of Aspidium, ${ }_{53},{ }_{5}^{8}$.

- - pinnæ of Aspidium, r59.

- - stem of Pinus, 187.

- - - Ranunculus, 2 I9.

- - tissues, 19.

Root-apex, 242, 243.

Root-cap, I53, I73, I90, 223, 242.

Root-hairs, r9o, 241.

Root-pressure, 228.

Roundness, 26r (Fig. 92).

Saccharomyces cerevisere. Lectures III. and IV., 23-34 (Fig. 5).

- - bud of, 25 .

- cell-wall of, 25 .

- holozoic, ro2.

- protoplasm of, 23.

- size of, 23 .
Saccharomyces cerevisea, nitrogen for, 102 .

source of

- - spore of, 25.

Saprophytes, 65 .

Scalariform tracheids, r49.

Scalesia, 275.

Scilla nutans. Lecture XXVII., 239249 (Figs. 86, 87, 88, 89).

- air-spaces of, 241 .

- - apex of, 242 .

- - bulb of, 240, 24I, 243.

- - carpels of, 246, 249 (Fig. 89).

- collenchyma of, 243 .

- conducting tract of, 24I, 243.

- cortex of root of, 24I, 243.

- cotyledon of, 248, 249.

- cuticle of leaves of, 245.

- - dissemination of seeds of, 248 .

- - embryo of, 248.

- - embryo-sac of, 246 (Fig. 89).

- endodermis of, 242.

- endosperm of, 248.

- epidermis of, $243,245$.

- fibrous layer of, 246.

- flowers of, 240, 245, 248, 249.

- fusion-nucleus of, 248 .

- gametophyte of, female, 247.

- - - male, 249.

- integument of, 248 .

- leaves of, 240, 245.

- life-history of, 248.

- loculus of, 246 (Fig. 89).

- megagametophyte of, 247.

- megaspores of, 249.

- microgametophyte of, 249.

- microspores of, 249.

- nucellus of, 246 (Fig. 89).

- ovary of, 246 (Fig. 89).

- ovules of, 246, 249 (Fig. 89).

- ovum of, $247,249$.

- passage-cells of, 242.

- perianth of, 246 .

- pericycle of, $24 \mathrm{I}$.

- piliferous layer of, $24 \mathrm{I}$.

- pollen-grains of, 246, 249 .

- pollen-sac of, 246, 249.

- pollen-tube of, 249 .

- - pollination of, 247 .

-- - protoxylem of, $24 \mathrm{I}$.

- root of, $240,241$.

- root-cap of, 242 .

- root-hairs of, $24 \mathrm{x}$.

- - seed-coat of, 248 .

- - seeds of, 248.

- - sperni-cells of, $247,249$.

- sporogenous cells of, 246 .

- stamens of, 246.

- - starch in, 243.

- stem of, 243 .

- stigmas of, $246,249$. 
Scilla nutans, stomata of, 243,245 .

- - styles of, 246 .

- - suspensor of, 248 .

- - syncarpous fruit of, 248.

Sclerenchyma, 152.

Section, optical, 6, 74, 89, 120.

Seed, $211,212$.

- of Pinus, 206.

- - Ranunculus, 230, 236.

- Scilla, 248.

Seed-coat of peas, 269 .

- - Pinus, 206, 209, 212.

- Ranunculus, 236.

- Scilla, 248.

Seed-cones, last three or more seasons, 204.

- of Pinus, 204.

- replace long shoots, 204 .

Seed-Plants, $2{ }_{3} 3$.

- and ferns distinguished, I78, $2 \mathrm{rr}$.

- sub-divisions of, 215 .

Seed-scale corresp onds to ligule, 205.

Seeds, transport of, 274 .

Segregation of determinants, $264,278$.

Selaginella martensii. Lecture XXI., I70-I 78 (Figs. 44, 45, 46, 47, 48, $49,150)$.

- - archegonium of, 176 .

- - antheridium of, I77.

- bast of, 173 .

- chloroplasts of, $\mathbf{1 7 2}$.

- conducting tract of, I73.

- - embryo of, 176 .

- endodermis of, 173 .

- - fertilisation of, $\mathbf{1 7 6 , 1 7 8 .}$

- - foot of, 176.

- gametophyte, female, 176.

_- - of, male, 175 .

- - germination of megaspores of, 175 .

- - - microspores of, 175 .

- heterosporous, I78.

- - leaves of, $x 71,173$.

- - ligule of, $17 x, 273$.

- - megasporangia of, I73.

- - megaspores of, 173 .

- microsporangia of, $\mathbf{1 7 3}$.

- microspores of, 173 .

- - ova of, 176.

- protoxylem of, $\mathbf{1 7 3}$.

- rhizophore of, I7I.

- root-cap of, 173 .

- sperms of, 175 .

- - sporangia of, I72, 173 .

- - sporophylls of, 173 .

- - stem of, 172.

- - suspensor of, 176 .

- - tapetum of, I74.

- - tracheids of, 173 .

Selection, natural, 277 .

Self, 262.
Semi-permeability, destruction of, $15, \mathbf{1 6}$, Semi-permeable membranes, I4. $[93,99$. Sepals, 230, 246.

Seta, 140.

Sexual reproduction, 39,72 , I09, IIO, III. - - and asexual reproduction contrasted, III, II2.

- hunger stimulus and, 40.

- - of Chlaniydomonas, 39.

- - Fucus, 107, 108, I09, IIo, III.

- - Mucor, 94, 95.

- - Penicillium, roo, ror.

- - Polysiphonia, I17-r2o.

- - Spirogyra, 72.

- - Vaucheria, 87, 88, 89.

- - Volvox, 79.

- - origin of, 40 .

Sheath of bundle or conducting tract.

- - Aspidium, 152.

- Ranunculus, 217, 218, 219.

Sieve-plates, I 50, 220.

Sieve-tubes, I5O, 220.

Sol, 21, 22.

Solution of crystalloids, II, I2, 2 I.

Smear-preparation, 27, 56, 64 .

Soma, an excrescence on germ-cells, 82 .

Somatic cells, $8 \mathrm{I}$.

- diploid, 255.

Sorus, I6r.

Souring of beer and wine, 65 .

- - milk, 65 .

Species, immutability of, $27 \mathrm{r}$.

- mutable, 276.

- origin of, 270 et segq .

- physiological, 65.

Spindle, achromatic, 252.

Spireme, 253.

Spirogyraporticalis. Lecture IX., 67-74

(Figs. II, 12).

- cell-division of, 70 .

- cell-wall, 68, 70, 7 r.

- - chloroplast of, 68, 69, 72.

- conjugation of, $70,71,73$.

- conjugation-tubes of, 73 .

- - holophytic, 69.

- - metabolism of, 69 .

- nucleus of, 69 .

- photosyntresis of, 69.

- - pyrenoids of, 69.

- - reconstruction of transverse section of, 68.

- reproduction of, 70,71 .

- - respiration of, 69.

- - sexual differentiation of, 73 .

- - sucker of, 7 I.

- waste products of, 69.

- zygospore of, 71, 72 .

Spongy parenchyma, I 55 .

Spontaneous generation, $5^{8}$.

Spore, 25, 26, 39. 
Spore of Aspidium, 163.

- Chlamydomonas, 39 .

- - Funaria, 136.

- Marchantia, 13r.

- - Mucor, 94.

- Penicillium, roo, ror.

- - Selaginella, 173 .

- - Vaucheria, 87, 89.

- - Volvox, 78.

- - yeast, 25, 26.

- resistance of, 26.

Spore-mother-cells, reduction mitosis in, $255,256,257$.

Sporogenous cells, derived from oosperm, 250.

- - of Aspidium, 163 .

- - Funaria, 140, 141 .

- - Marchantia, I3I.

- - Pinus, 201, 202, 206, 209.

- - Ranunculus, 23I, 234.

- - Scilla, 246.

- - - Selaginella, 173,174 .

- reduction-mitosis in, 255, 256, 257.

Sporophylls, 167, 173, 201, 212, 213, 236, 249.

Sporophyte, 142.

- diploid, 257.

- of Yolysiphonia (=Tetrasporic plant), Ir9.

Stains, acid and basic, for nuclei, 25 I.

Stamen (= microsporophyll), 231, 236,

Starch in Aspidium, r49.

- Chlamydomonas, 37, 4I, 43.

- Elodea canadensis, 43, 47.

- Marchantia, I24.

- Pinus, 182, 184, 195.

- Polysiphonia, II4.

- Ranunculus, 217, 220.

- Scilla, 243.

- Spirogyra, 69.

- Volvox, 78.

- a gel, 22.

"Steamer," 59.

Stereo-isomers, discovery of, 102.

Sterilisation, methods of, 59,60 .

- principles of, $56,58,59$.

Stigma, 233, 234, 235, 246.

Stimulus of gravity, 93, 94, 126.

- - hunger, 40.

- light, 38, 126.

Stomata, I40, 143, I54, I81, 216, 222, 243,245 .

Stomium, 162.

Streaming of protoplasm, 48 .

Style, 232, 234, 246.

Sub-cultures, 62.

Suberin, $\mathbf{x} 8$.

Sucrose, inversion of, 30,34 .

Surface actions of colloids, 21, 22.

Suspensions, 2 r.
Suspensor, 176, 208, 239, 248.

Sweetwort, 26.

Synapsis, 255.

Syncarpous truit, 248.

- ovary, 246 (Fig. 89).

Synergidæ, 234.

Synthetic forms, 170.

TANGENTIAL section, 188,197 .

Tapetum, r74, 231.

lartaric acid changed by Penicillium, IOI.

Tendrils of leguminous plants, 272.

Tetraspores, II6.

1 halamus, 230.

Thallophyta, I21, I33, I68.

Thallus, 122.

Theca, I40.

Torus of pit-membrane, I93 (Fig. 58).

Tracheids, 149, 173, 193, 195, 196, I97, I99, 221, 228.

Tradescantia virginiana, leaf-epidermis of, r, 4 (Fig. 2).

- root apex of, 242 (Fig. 87).

Transpiration current, 156, I57, 228.

- function of, $\mathbf{r}_{57}$.

Transport of organic substances, 116 .

- - stimuli, 77 .

Trichogyne, 117 .

Tube-cell of pollen-grain, 207, 235.

Turgor, I 5.

Tyndall, $5^{8}$.

ULTRA-MICROSCOPE, $2 \mathrm{x}$.

Unicellular plants, 75 .

Unisexual plants, xII.

Use and disuse, theory of, 277.

VACUOLES, 7, 14, 15, 18.

- contents of, 19.

- contractile, of Volvox, 77 .

- vacuoles of Chlamydomonas, 37,

Vagina, I40.

$\left[5^{1}, 5^{2}\right.$.

Vascular bundle. See Conducting tract.

Vaucheria sessilis. Lecture XI., 83-90 (Fig. r6).

- - antheridium of, 88.

- - asexual reproduction of, 87,89 .

- cell-wall of, $84,85,86$.

- chloroplasts of, 84,85 .

- cytoplasm of, 84 .

- division of labour in, 85 .

- - growth of, 86.

- nuclei of, $84,87,88$.

- - oil drops of, 85,86 .

- oogonium of, 88.

- photosynthesis of, 85 .

- - respiration in, 85 .

- - rhizoids of, 84,85 .

- - sexual reproduction of, $87,88,89$. 
Vaucheria sessilis, transverse septa in, 86. Water, needed for fertilisation, $\mathbf{I}_{30}, \mathbf{I}_{3} \mathbf{I}$,

- vacuole of, 84 .

- zoospore of, 87 .

Ventilating pores of Marchantia, 123.

Vessels, 22I, 228.

Vestigial structures, 273 .

Volvox aureus. Lecture X., 75-82 (Figs. I3, I4, I5).

- asexual reproduction of, 78 .

- cell-wall of, 76,77 .

- cilia of, 77 .

- - embryo of, development of, 79.

- - fertilisation of, $8 \mathbf{r}$.

- motion of, 76 .

- number of cells in, 76 .

- ova of, 8o.

- photosynthesis of, 78 .

- - protoplasmic connections of, 77 .

- reproductive cells of, $8 \mathbf{x}$.

- respiration of, 78 .

- - response to light of, 77 .

- sexual reproduction of, 79.

- - size of, 76 .

- somatic cells of, $8 \mathrm{r}$.

- sperms of, $8 \mathrm{o}$.

- - structure of, 76 .

WATER, cohesion-theory of ascent of, $\mathbf{1 6 3}, 224$ et seqq.

- cohesion of, in annulus, 163 .
138, $164,165,167,175$.

- path of, in Pinus, rgo.

- - - Ranunculus, 224.

- raised in Aspidium, 156.

- - Ranunculus, 224 et seqq.

- - trees, 227.

- tensile strength of, 224, 227.

- transmits pull, I56, 224 .

Wood (= xylem) of Aspidium, I49, 155 .

- of Pinus, 186.

- - Ranunculus, 217, 220.

- parenchyrna, I5I, I55, 22 I.

- primary, $\mathbf{1 8 6}, \mathbf{1} 87$.

- secondary, 186,187 .

- sections of, 195, 196, 197 .

-. spring and summer, 187 .

Wing of pine-seed, 206.

XANThOPhyLL, 46.

Xylem. See Wood.

YEAST. See Saccharomyces. Lectures III., IV., 23-34 (Fig. 5).

ZOOSPORES of Vaucheria, 87.

- Zygote of, 8, 265.

Zymase in yeast, 33 .

- Mucor, 96.

- - Penicillium, ror. 




\section{DAY USE}

RETURN TO DESK FROM WHICH BORROWED

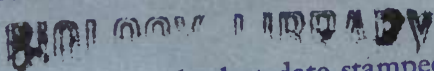

This book is due on the last date stamped below, or on the date to which renewed.

Renewed books are subject to immediate recall.

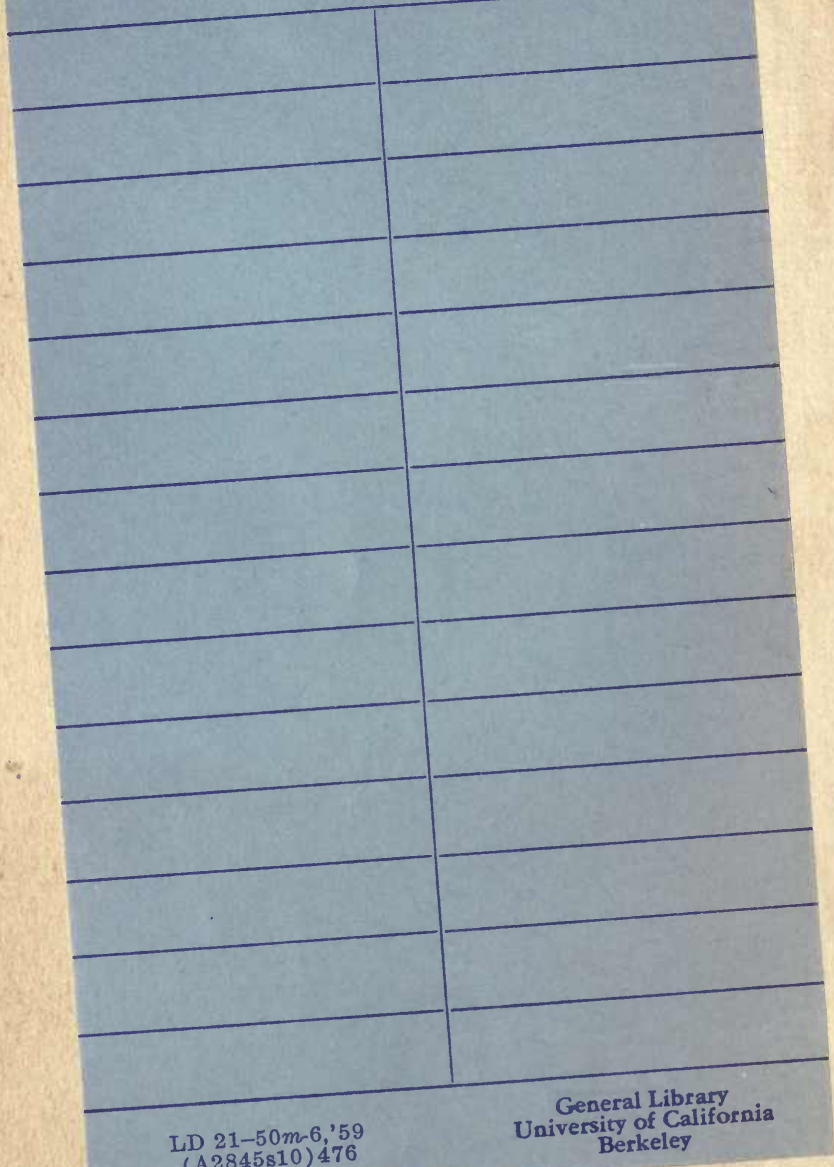

(A2845s10) 476

Berkeley 
U.C. BERKELEY LIBRARIES

U.C. BERKELEY LI

CD26082723

$-$
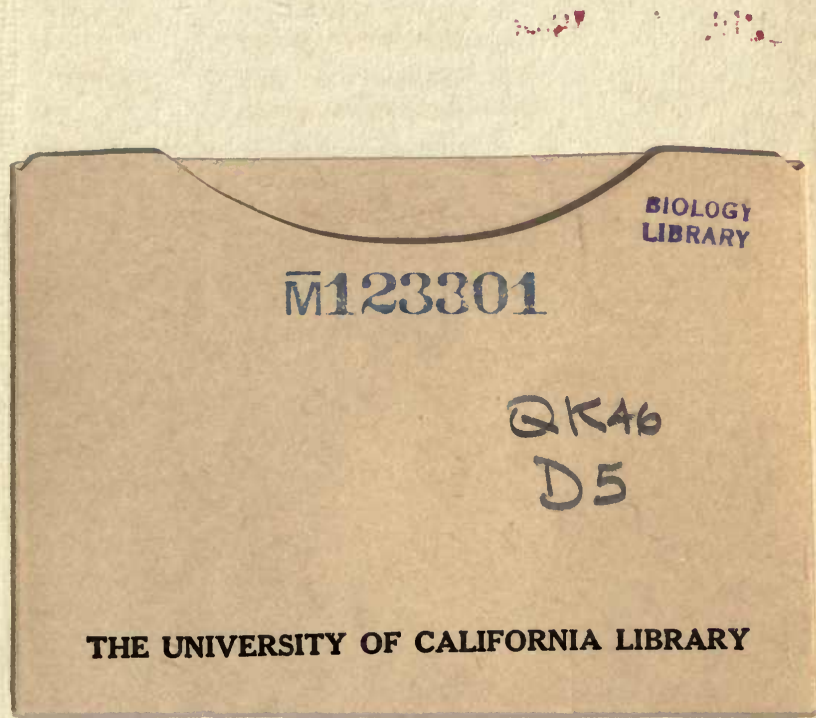
